\title{
Documentation for a Digital Computer Model of Nutrient and Dissolved-Oxygen Transport In the Truckee River and Truckee Canal Downstream from Reno, Nevada
}

By Jon O. Nowlin

U.S. GEOLOGICAL SURVEY

Open-File Report 87-554

Prepared in cooperation with the

CITIES OF RENO AND SPARKS, NEVADA

A product of the River-Quality

Assessment of the Truckee

and Carson River Basins,

Nevada and California

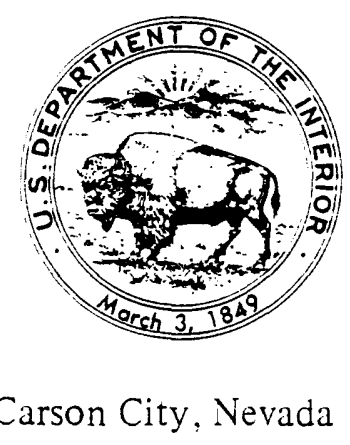


DEPARTMENT OF THE INTERIOR

DONALD PAUL HODEL, Secretary

U.S. GEOLOGICAL SURVEY

Dallas L. Peck, Director

For additional information

Copies of this report may be write to: purchased from:

U.S. Geological Survey

Room 227, Federal Building

705 North Plaza Street

Carson City, NV 89701
U.S. Geological Survey

Books and Open-File Reports Section

Federal Center

Box 25425

Denver, CO 80225 


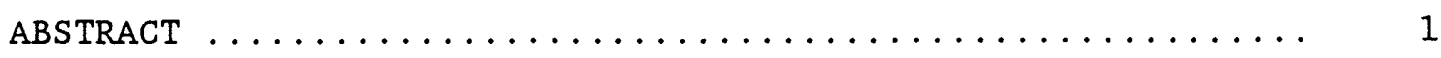

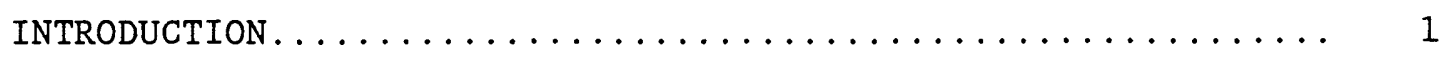

COMPUTER PROGRAM.......................... 4

DATA REQUIREMENTS ......................... 4

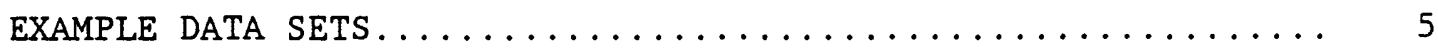

EXAMPLE MODEL OUTPUT. $\ldots \ldots \ldots \ldots \ldots \ldots \ldots \ldots \ldots \ldots \ldots \ldots$

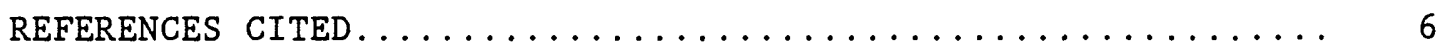

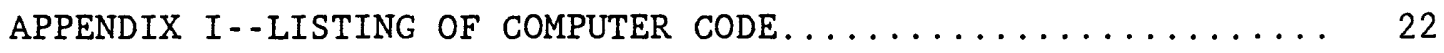

APPENDIX II--LISTING OF EXAMPLE DATA SETS........... 73

A. Truckee River, June $1979 \ldots \ldots \ldots \ldots \ldots \ldots \ldots \ldots . \ldots \ldots$

B. Truckee River, August $1979 \ldots \ldots \ldots \ldots \ldots \ldots \ldots$

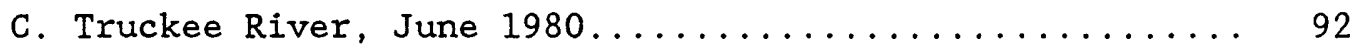

D. Truckee River, August $1980 \ldots \ldots \ldots \ldots \ldots \ldots \ldots \ldots \ldots \ldots$

E. Truckee Canal, June 1979.................... 109

F. Truckee Canal, August $1979 \ldots \ldots \ldots \ldots \ldots \ldots \ldots \ldots \ldots \ldots$

G. Truckee Cana1, June $1980 \ldots \ldots \ldots \ldots \ldots \ldots \ldots \ldots \ldots \ldots$

H. Truckee Canal, August $1980 \ldots \ldots \ldots \ldots \ldots \ldots \ldots \ldots \ldots$

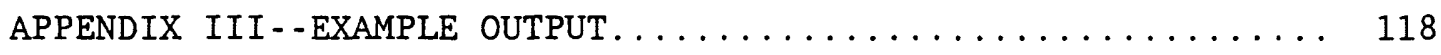

\section{TABLE}

Table 1. Specifications for input data.............. 8

ILLUSTRATION

Figure 1. Map showing location and general features of the modeled reach of the Truckee River and Canal..... 2 


\begin{tabular}{|c|c|c|}
\hline Multiply Inch-pound unit & by & to obtain metric unit \\
\hline \multicolumn{3}{|c|}{ Length } \\
\hline $\begin{array}{l}\text { foot (ft) } \\
\text { lnch (In.) } \\
\text { mlle (ml) }\end{array}$ & $\begin{array}{l}0.3048 \\
25.40 \\
1.609\end{array}$ & $\begin{array}{l}\text { meter (m) } \\
\text { millimeter (mm) } \\
\text { kilometer ( km) }\end{array}$ \\
\hline \multicolumn{3}{|c|}{ Area } \\
\hline $\begin{array}{l}\text { acre } \\
\text { acre } \\
\text { square foot }\left(f t^{2}\right) \\
\text { square mile }\left(m l^{2}\right)\end{array}$ & $\begin{array}{l}47 \\
0.4047 \\
0.09294 \\
2.590\end{array}$ & $\begin{array}{l}\text { square meter }\left(\mathrm{m}^{2}\right) \\
\text { hectare } \\
\text { square meter }\left(\mathrm{m}^{2}\right) \\
\text { square k1lometer }\left(\mathrm{km}^{2}\right)\end{array}$ \\
\hline \multicolumn{3}{|c|}{ Volume } \\
\hline acre-foot (acre-ft) & 0.001233 & $\begin{array}{l}\text { cubic meter }\left(\mathrm{m}^{3}\right) \\
\text { cublc kilometers }\left(\mathrm{km}^{3}\right)\end{array}$ \\
\hline \multicolumn{3}{|c|}{ Velocity } \\
\hline foot per second $(\mathrm{ft} / \mathrm{s})$ & 0.3048 & meter per second $(\mathrm{m} / \mathrm{s})$ \\
\hline \multicolumn{3}{|c|}{ Flow } \\
\hline $\begin{array}{l}\text { cubic foot per second }\left(\mathrm{ft}^{3} / \mathrm{s}\right) \\
\text { million gallons per day ( } \mathrm{Mgal} / \mathrm{d}) \\
\text { pound per day (lb/day) }\end{array}$ & $\begin{array}{l}0.02832 \\
0.04381 \\
0.4556\end{array}$ & $\begin{array}{l}\text { cublc meter per second }\left(\mathrm{m}^{3} / \mathrm{s}\right) \\
\text { cubic meters per second }\left(\mathrm{m}^{3} / \mathrm{s}\right) \\
\text { kilograms per day }\end{array}$ \\
\hline \multicolumn{3}{|c|}{ Mass } \\
\hline $\begin{array}{l}\text { pound, avoirdupois ( } 1 \mathrm{~b}) \\
\text { tons }\end{array}$ & $\begin{array}{l}28.35 \\
0.9072\end{array}$ & $\begin{array}{l}\operatorname{gram}(g) \\
\text { metric tons }(t)\end{array}$ \\
\hline \multicolumn{3}{|c|}{ Spectific Conductance } \\
\hline $\begin{array}{l}\text { micromhos per centimeter } \\
\text { at } 25{ }^{\circ} \mathrm{C} \text { (micromhos) }\end{array}$ & 1.000 & $\begin{array}{l}\text { microsiemens per centimeter } \\
\text { at } 25{ }^{\circ} \mathrm{C} \text { (microsiemens; } \mu \mathrm{S} \text { ) }\end{array}$ \\
\hline
\end{tabular}

For temperature, degrees Celsius $\left({ }^{\circ} \mathrm{C}\right)$ can be converted to degrees Fahrenheit $\left({ }^{\circ} \mathrm{F}\right)$ by using the formula ${ }^{\circ} \mathrm{F}=\left[(1.8)\left({ }^{\circ} \mathrm{C}\right)\right]+32$.

Sea level: In this report, "sea level" refers to the National Geodetic Vertical Datum of 1929 (NGVD of 1929), which is derived from a general adjustment of the first-order leveling networks of both the United States and Canada. 
DOCUMENTATION FOR A DIGITAL COMPUTER MODEL OF

NUTRIENT AND DISSOLVED-OXYGEN TRANSPORT

IN THE TRUCKEE RIVER AND TRUCKEE CANAL

DOWNSTREAM FROM RENO, NEVADA

By Jon 0. Nowlin

\begin{abstract}
A digital water-quality model was constructed as part of a waterquality assessment of the Truckee River downstream from Reno. This report provides documentation on the computer code and the principal data sets used in model calibration, validation, and simulations.

\section{INTRODUCTION}

As part of a water-quality assessment of the Truckee River in Nevada (Nowlin and others, 1980; Brown and others, 1985; Nowlin, 1987), a digital water-quality model was constructed for the Truckee River downstream of Reno to Marble Bluff Dam, and the length of the Truckee Canal from Derby Dam to Lahontan Reservoir (figure 1). The model was calibrated against four independent data sets compiled from intensive field investigations conducted in 1979-81 (LaCamera and others, 1985). A detailed description of the construction and calibration of the computer model has been published, along with the results of selected simulations of river and canal quality in response to alternative levels of waste treatment at the Reno-Sparks sewage treatment plant (Nowlin, 1987). This report documents the computer code used for those simulations and presents eight calibrated data sets used as the basis for published model simulations. This report is not designed to be a self-contained user quide to application of the TRWQ Model; such applications will require reference to appropriate sections and data tables of the previous report (Nowlin, 1987) in addition to the information in this report.

The Truckee River Water-Quality Model (TRWQ Mode1) is a onedimensional, steady-state, digital model capable of simulating concentrations of dissolved solids, ultimate carbonaceous biochemical oxygen demand, dissolved oxygen, nitrogen species (organic-, ammonia-, nitrite-, nitrate nitrogen), orthophosphorus, and total phosphorus. The model also simulates specific electrical conductance as a surrogate for measured dissolved solids. 


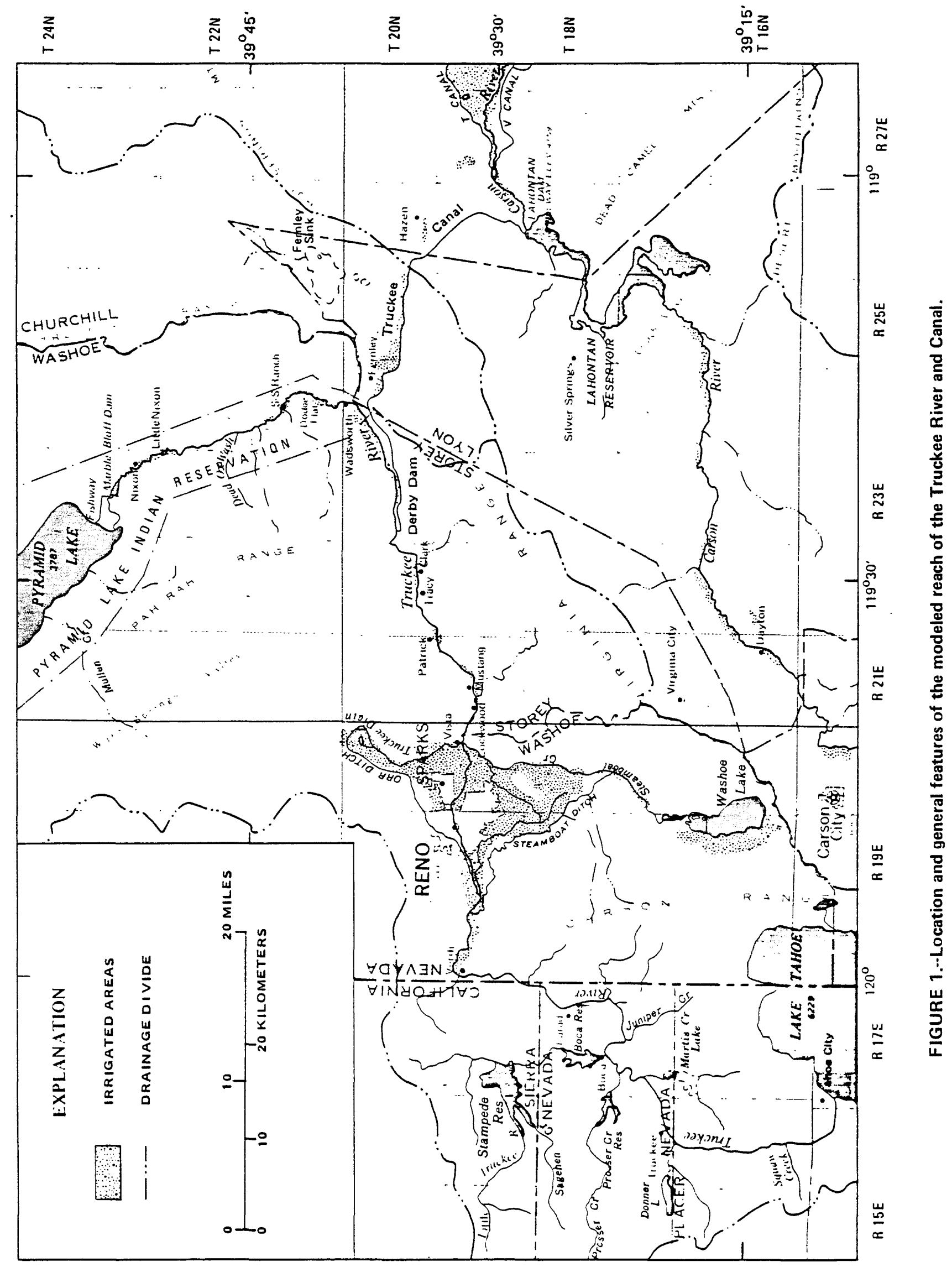


Model results are computed in terms of mean daily values for the predicted variables. Results may be obtained in both tabular and graphic form. The model was configured to simulate water quality in a $56-\mathrm{mile}$ reach of the Truckee River from McCarran. Bridge on the east side of Reno to Marble Bluff Dam, just above the mouth of the river at Pyramid Lake, and in the 34-mile reach of the Truckee Canal from the diversion of water from the Truckee River to the drop structure at the end of the canal into Lahontan Reservoir. Configuration of the mainstem Truckee River model includes short submodels of North Truckee Drain and Steamboat Creek, the two perennial tributaries to the river downstream from Reno.

The model includes consideration of diversions from the river and canal, tributary inflows, and separate nonpoint agricultural returns and ground-water inflows along the modeled reaches. Running the model requires specification of stream-flow and water-quality data for the start of the modeled reaches, for any contributing tributaries, and for nonpoint return flows. At modeled points of diversions, mean daily diverted flows must be specified. Channel seepage losses may be linearly distributed over a modeled reach.

The TRWQ Model simulates mean daily concentrations of constituents at specified increments along the length of the modeled river reach. Simulated parameters include:

Conservative variables (mass balance of all inputs and losses):

Water discharge

Dissolved solids

Specific conductance (used as an easily-measured surrogate of dissolved solids)

Nonconservative variables (mass balance of all inputs and losses coupled with first-order transformation and decay algorithms):

\author{
Ultimate carbonaceous biochemical oxygen-demand ( $C B O D u$ ) \\ Organic nitrogen \\ Ammonia nitrogen \\ Nitrite nitrogen \\ Nitrate nitrogen \\ Orthophosphorus (two values, see Appendix II) \\ Total phosphorus \\ Ratio of inorganic nitrogen to orthophosphorus \\ Reaeration rate \\ Dissolved oxygen
}


Discharges from point sources and results from tributary submodels are mass-balanced at the start of model subreaches. Diversions are treated as negative point sources. Nonpoint surface returns (agricultural return flows) and ground-water inflows are modeled independently and are linearly distributed along the length of receiving subreaches. Concentrations of nonpoint returns are specified, or may be calculated as a percentage of the river instream concentrations at the point of diversion for a specific return.

\section{COMPUTER PROGRAM}

The computer code used in the TRWQ Model simulations is listed in Appendix I. The code is an adaptation of a model previously described by Bauer and others (1979). The basic equations and assumptions used in development of the computer program are discussed both by Bauer and others and in Nowlin (1987). Modifications made to the original computer code are discussed in Nowlin and are referenced within the program listing in Appendix I.

The program is written in FORTRAN-77 computer language. Model calibration, validation, and simulations were performed with the program compiled on a PRIME model 9955-II minicomputer under the PRIMOS operating system. [1]

\section{DATA REQUIREMENTS}

Model calculations are performed on data read from an input data set formatted as 80-column "card images". Running the model requires an appropriate data set specifying all the input parameters to be read by the compiled FORTRAN program; the version listed in Appendix I assumes the input data sets in files on a PRIME computer. Output is controlled in volume and format by the user-specified options in the input data set.

The listing in Appendix $I$ is designed to write 132 -column lines of information to output files, in both tabular and graphic form, suitable for printing on a standard line printer. Full outputs include documentation of all input data, simulated water-quality constituents, and other pertinent model parameters including traveltimes, reaction coefficients at ambient temperatures, channel-geometry, and observed water-quality data.

Input data-set requirements are given in table 1 . Annotations in the table indicate various model options and coding for execution or suppression of those options. More importantly, notes in the table indicate those data input parameters with values specific to the calibrated and validated version of the TRWQ Model. Deviation from those values may produce results that cannot be directly compared to those previously published.

[1] The use of brand names within this report is for indentification purposes only and does not imply endorsement by the U.S. Geological Survey. 
Listings of example data sets used as the basis for published TRWQ Model simulations are presented in Appendix II. These eight data sets include final calibrated and validated model parameters and input data reflecting conditions observed in the Truckee River and Truckee Canal during extensive field investigations in June 1979, August 1979, June 1980, and August 1980.

Details on configuration of the TRWQ Model to the modeled reach of the Truckee River and Canal, data reduction for modeling, assumptions used in model calibration and validation, the results of simulations, and discussions on limitations of model applications have been published in great detail (Nowlin, 1987) and should be referenced before attempting to run the model. Deviations from the published configuration of the model to represent the physical river and canal systems, the published rate constants derived from model calibration and validation, and the published assumptions used in formulating simulations may produce results not directy comparable to those previously published.

\section{EXAMPLE MODEL OUTPUT}

An example of printed output from a model run is presented in Appendix III. The tables and graphs are the result of running the river data set for the August 1979 conditions presented in Appendix II-B. Included in the example are varying format controls as explained in Appendix III. 


\section{REFERENCES CITED}

Bansal, M. K., 1973, Atmospheric reaeration in natural streams: Water Research, v. 7., no. 5., p. 769-782.

Bauer, D. P., Jennings, M. D., and Miller, J. E., 1979, One-dimensional, steady-state, stream-quality model: U.S. Geological Survey Water-Resources Investigations Report 79-45, $215 \mathrm{p}$.

Bennett, J. P., and Rathbun, R. E., 1972, Reaeration in open-channel flow: U.S. Geological Survey Professional Paper 737, 75 p.

Brown, W. M. III, Nowlin, J. O., Smith, L. H., and Regan, M. O., 1986, River-quality assessment of the Truckee and Carson River system, California and Nevada--hydrologic characteristics: U.S. Geological Survey Open-File Report 84-576, 201 p.

Elmore, H. L. and West, W. F., 1961, Effects of water temperature on stream reaeration: Journal of Sanitary Engineering Division, v. 87, no. SA6, p. 59 .

LaCamera, R. L., Hoffman, R. J., Nowlin, J. O., Smith, L. H., and Lima, S.M., 1985, Data on surface-water quality and quantity, Truckee River system, California and Nevada, 1979-81: U.S. Geological Survey Open-File Report 84-238, 189 p.

Langbein, W. B., and Durum, W. H., 1967, The aeration capacity of streams: U.S. Geological Survey Circular 542, 6 p.

McCutcheon, S. C., and Jennings, M. E., 1982, Stream reaeration by Velz method: Journal of Environmental Engineering, v. 188, no. EE1, p. $218-221$.

Nowlin, J. 0., 1987, Modeling nutrient and dissolved-oxygen transport in the Truckee River and Truckee Canal downstream from Reno, Nevada: U.S. Geological Survey Water-Resources Investigations Report 87-4037, 487 p.

Nowlin, J. O., Brown, W. M. III, Smith, L. H., and Hoffman, R.J., 1980, Planning and design of studies for river-quality assessment in the Truckee and Carson River basins, California and Nevada: U.S. Geological Survey Open-File Report 80-435, 75 p.

O'Conner, D. J., and Dobbins, W. E., 1958, Mechanisms of reaeration in natural streams: Transactions American Society of Civil Engineers, v. 123, p. 641-684.

Padden, T. J., and Gloyna, E. F., 1971, Simulation of stream processes in a model river: University of Texas, Austin, Report EHE-70-23, CRWR-72, 130 p. 
Parkhurst, J. D., and Pomeroy, R. D., 1972, Oxygen absorption in streams: Journal of Sanitary Engineering Division, v. 98, no. SA1, p. 101-124.

Shindala, Adnan, 1972, Mathematical modeling for water-quality management in streams and estuaries: Mississippi State University Department of Civil Engineering, $62 \mathrm{p}$.

Thackston, E. L., and Krenkel, P. A., 1969, Reaeration prediction in natural streams: Journal of Sanitary Engineering Division, v. 95, no. SA-1, p. 65-94.

Thurston, R. V., Russo, R. C., and Emerson, K., 1974, Aqueous ammonia equilibrium calculations: Fisheries Bioassay Laboratory, Montana State University, Bozeman, Technical Report No. 74-1, 18 p. plus appendix.

Tsivoglou, E. C., and Wallace, J. R., 1972, Characterization of stream reaeration capacity: U.S. Environmental Protection Agency Ecological Research Series Report EPA-R3-72-012, 317 p.

Weiss, C. M., 1970, Solubility of hydrogen, oxygen, and argon in water and seawater: Deap-Sea Research, v. 17, p 721-735.

Velz, C. J., 1970, Applied stream sanitation: New York, John Wiley, $619 \mathrm{p}$. 
TABLE 1.--Specifications for input data.

[The following material gives instructions for coding input data required to run the TRWQ Model. For a complete discussion of model variables and prodedures used for model calibration and validation, refer to Nowlin (1987).

Model input is in the form of 80-column card images. Card input consists of three groups for each run:

(1) "A" cards specifying basic options for the entire run,

(2) " 1 " through "9" cards, as required, for North Truckee Drain and Steamboat Creek submodels, and

(3) "1" through "9" cards, as required for the mainstem.

Unless otherwise stated below, all cards and fields on a card are required. Several parameters have alternative options for units of entry or methods of calculation. Alternatives NOT USED in the calibrated Truckee River data sets presented in Appendix 1 are so indicated.]

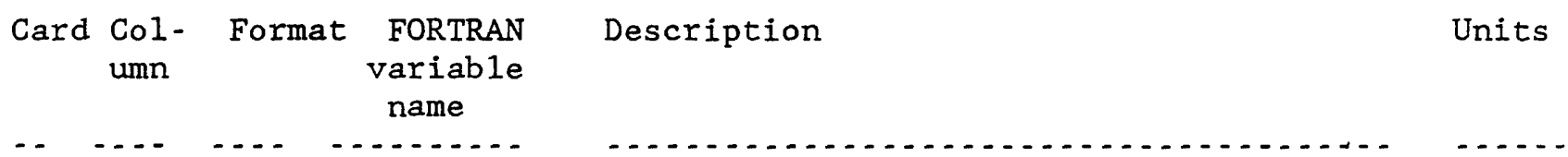

"A1" Card-- Primary model options (required, 1 card at beginning of each -..... data set)

A1 $1-2 \quad 2 \mathrm{~A} 1 \quad \cdots \quad$ Card type $=\mathrm{A} 1$

A1 3-9 $\quad$ - $\quad \ldots \quad$ leave blank

A1 $10 \quad$ I1 $\quad$ KPLOT

Scaling for line-printer plots

0 (or b1ank)= autoscale to multiples of 1,2 , or 5 ;

$1=$ autoscale to maximum and minimum values;

3- use internal fixed scales.

A1 11 I1 IPNET

Photosynthesis and respiration effects on mean daily DO (see Nowlin, 1987, and "51" cards below)-.

$1=$ model coded $P$ as net $P \& R$ (normal option),

$2=$ model coded $R$ only (use to calibrate minimum daily DO (see Appendix II and Nowlin, 1987)
A1 13 I1 ITEST
Print input cards - 9= yes. (used to check input data deck, normally left blank). 
TABLE 1.--Specifications for input data--Continued.

"1" Cards-- Output titles to appear at head of tables and figures (optional). -.......
$11 \quad 1-2 \quad$ I2
Card type $=11$
113
$-$
leave blank
$11 \quad 4-79 \quad 19 A 4$
TITLE1 (19)
Title for output pages, 1st line. User-defined title to appear at top of all printed output.
$12 \quad 1-2 \quad$ I2
Card type $=12$
12
leave blank
$12 \quad 4-79 \quad 19 A 4 \quad$ TITLE2(19)
Title for output pages, 2nd line. Second user-defined line.
"21" Cards-- Output format controls (required). The following cards provide ....... control of format and volume of tables and plots.
$21 \quad 1-2$
I2
Card type $=21$
$21 \quad 3$
$-$
$-$
leave blank
214
I1 ITAB1
Option 1: Control on printing of tables 3-7: The following values determine what is to be included in the printed output:
0 (or blank) $=$ all tables, or
3= table 3, segment environmental controls,
4= table 4, water-quality inputs,
$5=$ table 5 , segment hyraulics data,
$6=$ table 6 , segment reaction rates,
$7=\operatorname{table} 7$, observed water-quality data,
$8=$ all tables except table 5,
$9=$ no tabular output

$\begin{array}{llll}21 & 5 & \text { I1 } & \text { ITAB2 }\end{array}$

Option 2 for printing tables 8 and 9 , model results (full table has predicted concentrations for each calculation interval and predicted loads at segment ends, short 
TABLE 1.--Specifications for input data--Continued.

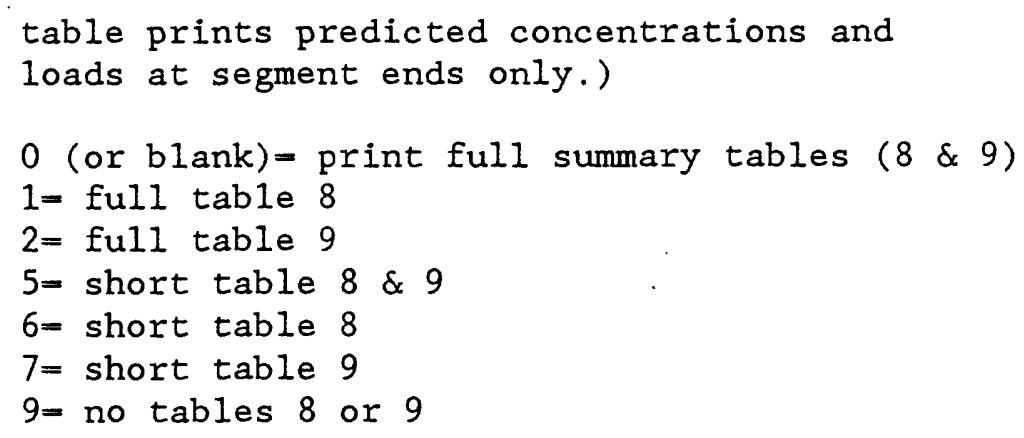

\section{6 \\ $21 \quad 7-8$ \\ I2 IPLOT}

219

$21 \quad 10-11$ I2 NSEG

$21 \quad 13-14$ I2 JSEG

$21 \quad 15-19--$

$21 \quad 20-27$ F8.0 XSTRT leave blank

Option for controlling output of plots on line printer.-

0 (or blank) $=$ all plots

$1=$ reaeration rate and channel geometry,

$2=\mathrm{CBODu}$,

$3=$ Nitrogen cycle,

$4=$ DO concentrations, deficits, \& saturation,

$5=$ orthophosphorus,

$6=$ conservatives,

$7=$ nonconservatives.

\section{leave blank}

Number of segments for modeled reach:

Submode1 1 (N. Truckee Drain) $=02$

Submodel 2 (Steamboat Creek) $=02$

Mainstem model for river $=43$

Truckee Canal =

09

leave blank

FOR SUBMODELS ONLY, leave blank for mainstem model: mainstem segment to receive results of tributary submodel: Submodel 1 (N. Truckee Drain) $=02$ Submodel 2 (Steamboat Creek) $=03$ Mainstem model for river and canal $=00$

leave blank

Starting river miles from mouth. Submodel 1 (N. Truckee Drain) $=00.26$ Submodel 2 (Steamboat Creek) $=00.75$ Mainstem model for river $=56.12$ Truckee Canal 
TABLE 1.--Specifications for input data--Continued.

$\begin{array}{llllll}21 & 28 & \ldots & \ldots & & \end{array}$

$21 \quad 29-36$ F8.0 XEND

Ending river mile modeled.

mi

$21 \quad 37-39 \quad-$.

leave blank

$21 \quad 40 \quad$ I1 $\quad$ IGRAPH

Option for outputing file for graphics-- -.

$0($ or blank $)=$ no output file,

$1=$ outputs a file for plotting results

(file name will be PO. [data set name]).

"22, 23" Cards-- (Required) Upstream boundary conditions at start of

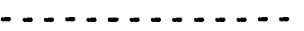
modeled reach. Code starting data for simulation for North Truckee Drain and Steamboat Creek submodels, and mainstem river. Data used in model calibration and published simulations are given in Nowlin (1987) and the data sets in Appendix 2.

22 1-2 I2 $\quad--\quad \quad$ Card type $=22$

$\begin{array}{lllll}22 & 3 & \ldots & \ldots & \text { leave blank }\end{array}$

22 4-11 F8.0 STARTC(1) Starting discharge 3

$22 \quad 12-19$ F8.0 $\operatorname{STARTC}(2)$

Starting temperature $\mathrm{ft} / \mathrm{s}$

$22 \quad 20-27 \quad$ F8.0 STARTC(3)

Starting barometric pressure

$\circ \mathrm{C}$

$22 \quad 28-35$ F8.0 STARTC(4)

Starting specific conductance

$\mathrm{mm} \mathrm{Hg}$

$22 \quad 36-43 \quad$ F8.0 STARTC(5)

Starting dissolved oxygen

umhos

$22 \quad 44-51$ F8.0 STARTC(9)

Starting CBODu

$\mathrm{mg} / \mathrm{L}$

$22 \quad 52-59 \quad--\quad-\cdot$

leave blank

22 60-67 F8.0 STARTC(11) Starting organic-N

22 68-75 F8.0 STARTC(12) Starting ammonia-N

$23 \quad 1-2 \quad$ I2

$23 \quad 3$

$23 \quad 4-11 \quad$ F8.0 STARTC (13)

$23 \quad 12-19$ F8.0 STARTC (14)

\section{Card type $=23$}

leave blank

Starting nitrite-N

Starting nitrate $-\mathrm{N}$
mg/L

$\mathrm{mg} / \mathrm{L}$

$\mathrm{mg} / \mathrm{L}$

$-\cdot$

$\mathrm{mg} / \mathrm{L}$

mg/L 
TABLE 1.--Specifications for input data--Continued.

\begin{tabular}{|c|c|c|c|c|c|}
\hline 23 & $20-27$ & F8. 0 & $\operatorname{STARTC}(16)$ & Starting orthophosphorus - A & $\mathrm{mg} / \mathrm{L}$ \\
\hline 23 & $28-35$ & F8. 0 & STARTC (17) & Starting specific conductance & umhos \\
\hline 23 & $36-43$ & F8. 0 & $\operatorname{STARTC}(18)$ & Starting dissolved solids & $\mathrm{mg} / \mathrm{L}$ \\
\hline 3 & $44-51$ & -- & STARTC (19) & leave blank & \\
\hline 23 & $52-59$ & F8.0 & $\operatorname{STARTC}(20)$ & Starting orthophosphorus - B & $\mathrm{mg} / \mathrm{L}$ \\
\hline 23 & $60-67$ & F8. 0 & $\operatorname{STARTC}(21)$ & Starting phosphorus (total) & $\mathrm{mg} / \mathrm{L}$ \\
\hline \multicolumn{6}{|c|}{$\begin{array}{l}\text { "31" Cards-- Segment descriptions, one card required for each modeled } \\
\text { segment. Cards must be coded exactly as shown in } \\
\text { Appendix II to replicate published simulations in Nowlin (1987). }\end{array}$} \\
\hline 31 & $1-2$ & I2 & -- & Card type $=31$ & -- \\
\hline 31 & $3-4$ & I2 & $\mathrm{N}$ & Segment number, starting with 1 & -- \\
\hline 31 & 5 & $\cdots$ & -- & leave blank & - \\
\hline 31 & $6-29$ & $6(\mathrm{~A} 4)$ & $\operatorname{SNAME}(N)$ & $\begin{array}{l}\text { Segment name (optional) - names to identi } \\
\text { segments on tabular outputs. }\end{array}$ & \\
\hline 31 & $30-35$ & F6. 0 & $\operatorname{BSEG}(N)$ & Segment starting river mile & mi \\
\hline 31 & 36 & -- & -- & leave blank & -- \\
\hline 31 & $37-80$ & $11 \mathrm{~A} 4$ & $\operatorname{SDESC}(\mathrm{N})$ & $\begin{array}{l}\text { Segment description (optional) - } \\
\text { pertinent descriptive material to be } \\
\text { printed in output table } 2 \text {. }\end{array}$ & -- \\
\hline
\end{tabular}


TABLE 1.--Specifications for input data--Continued.

"41" Cards-- Channel geometry data (required). In order to duplicate ....... calibrated TRWQ Model results (Nowlin, 1987), the following cards should be coded exactly as indicated in Appendix II.

Channel geometry data (mean values for segments) may be specified, or calculated by the program as a function of discharge by providing coefficients for the relationship $\mathrm{XB}$

$X=X A Q$, where $X A$ and $X B$ are coefficients for the exponential relationship between $X$ and $Q$ (discharge). Whichever method is used, input must be made for exactly two parameters in combinations of either:

$$
\begin{aligned}
& \text { (a) (velocity or traveltime or area) and } \\
& \text { (depth or width) }
\end{aligned}
$$

(b) width and depth.

From these two parameters and the specified segment discharge, all other channel-geometry parameters will be calculated and printed in table 5 .

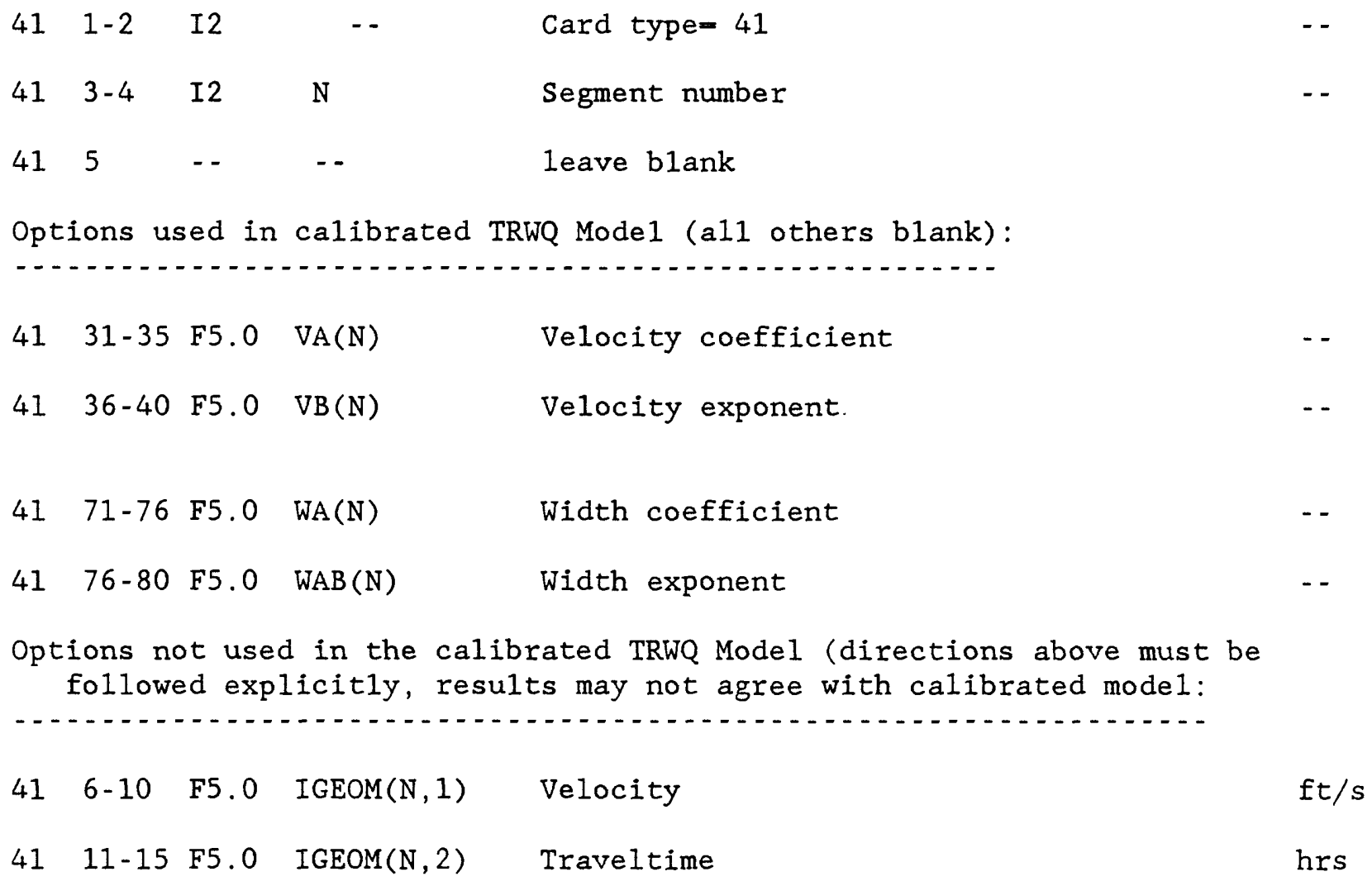


TABLE 1.--Specifications for input data--Continued.

$\begin{array}{lllll}41 & 16-20 & F 5.0 & \operatorname{IGEOM}(\mathrm{N}, 3) & \text { Cross-sectional area } \\ 41 & 21-25 & F 5.0 & \operatorname{IGEOM}(\mathrm{N}, 4) & \text { Mean depth } \\ 41 & 26-30 & \text { F5.0 } & \operatorname{IGEOM}(\mathrm{N}, 5) & \text { Mean cross-sectional width } \\ 41 & 41-45 & F 5.0 & \mathrm{TA}(\mathrm{N}) & \text { Traveltime coefficient } \\ 41 & 46-50 & \mathrm{~F} 5.0 & \mathrm{~TB}(\mathrm{~N}) & \text { Traveltime exponent } \\ 41 & 51-55 & \mathrm{~F} 5.0 & \mathrm{AA}(\mathrm{N}) & \text { Area coefficient } \\ 41 & 56-60 & \mathrm{~F} 5.0 & \mathrm{AB}(\mathrm{N}) & \text { Area exponent } \\ 41 & 61-65 & \mathrm{~F} 5.0 & \mathrm{DA}(\mathrm{N}) & \text { Channel depth coefficient } \\ 41 & 65-70 & \mathrm{~F} 5.0 & \mathrm{DB}(\mathrm{N}) & \text { Channel depth exponent }\end{array}$

"51" Cards-- (Required). Segment environmental controls for dissolved-oxygen ........ saturation and un-ionized ammonia (1 card required for each modeled segment, specify average values for the segment).
$51 \quad 1-2 \quad$ I2 $\quad-$
Card type $=51$
$51 \quad 3-4 \quad$ I2 $\quad \mathrm{N}$
Segment number

The following values are to be varied according to the specific environmental conditions modeled; changes from those documented for the TRWQ Model calibration and applications (Nowlin, 1987) will affect the resultant simulations

\begin{tabular}{|c|c|c|c|c|c|}
\hline 51 & $5-9$ & F5. 0 & $\operatorname{TEMP}(\mathrm{N})$ & Temperature & $\circ \mathrm{C}$ \\
\hline 51 & $10-14$ & F5. 0 & $\mathrm{BP}(\mathrm{N})$ & Barometric pressure & $\mathrm{mm} \mathrm{Hg}$ \\
\hline 51 & $15-19$ & F5. 0 & $\operatorname{SCOND}(\mathrm{N})$ & $\begin{array}{l}\text { Approximate specific conductance } \\
\text { (used for salinity corrections for DO } \\
\text { calculations) }\end{array}$ & umhos \\
\hline 51 & $20-25$ & F6. 0 & $\mathrm{BN}(\mathrm{N})$ & $\begin{array}{l}\text { Benthic DO demand at } 20 \mathrm{deg} C \\
\text { (not used to calibrate TRWQ Model) }\end{array}$ & $\mathrm{g} / \mathrm{m}^{\wedge} /$ day \\
\hline 51 & $26-31$ & $\mathrm{~F} 6.0$ & $\operatorname{PNET}(\mathrm{N})$ & $\begin{array}{l}\text { Photosynthetic DO production [enter net } \\
(P-R) \text { if } R \text { is to be used to calibrate } \\
\text { daily DO (see text)] }\end{array}$ & $\mathrm{mg} / \mathrm{L} / \mathrm{day}$ \\
\hline
\end{tabular}


TABLE 1.--Specifications for input data--Continued.

$51 \quad 32-37 \quad F 6.0 \quad \operatorname{RESP}(\mathrm{N})$

$51 \quad 38-43 \quad-$

$5144-49^{\circ} \quad \mathrm{K} 2(\mathrm{~N})$

$5150-51$ I2 IK2(N)

$5152-56 \quad \operatorname{SFPM}(\mathrm{N})$

$51 \quad 57-64 \quad \operatorname{SLOPE}(\mathrm{N})$

$51 \quad 65$

$5166-68$ F3.0 $\mathrm{PH}(\mathrm{N})$

$\begin{array}{llll}51 & 69 & - & -\end{array}$

$5170-75$ F6.0 CTSVIG(N)
Respiratory (plant) DO demand (used as factor for minimum daily DO, see Appendix II and Nowlin, 1987)

leave blank

Reaeration coefficient at 20 deg C; leave 1/day blank if reaeration coefficient to be calculated from channel geometry. NOT USED for the calibrated TRWQ Mode1.

Reaeration equation applied to segment; leave blank if reaeration coefficeint specified above. Equation 12 was used in TRWQ Model calibration, use of other equations will affect the resultant simulations.

Equation

K2 calculated as function of:

1 Bennett-Rathbun

$\mathrm{V}, \mathrm{D}, \mathrm{S}$

2 Velz

3 Langbein-Durum

D

$\mathrm{V}, \mathrm{D}$

Padden-Gloyna

$\mathrm{V}, \mathrm{D}$

Bansal

$\mathrm{V}, \mathrm{D}$

Parkhurst-Pomeroy

7 Tsivoglou

12 Tsivoglou calibrated for

Truckee River tracer data V, S

Channel slope (required as indicated

$\mathrm{ft} / \mathrm{mi}$ above)

Channel slope

$f t / f t$

leave blank

mean reach $\mathrm{pH}$ (used for un-ionized ammonia computations

leave blank

Optional escape coefficient for equation -7 above $(\mathrm{K} 2=\mathrm{CxVxS})$. Default coefficient is $\mathrm{C}=4100$ for velocity in $\mathrm{ft} / \mathrm{s}$ and slope in $f t / f t$, as published by Tsivoglou and Wallace, 1972 (NOT USED in calibrated TRWQ Model). 
TABLE 1.--Specifications for input data--Continued.

"61" Cards-- (Required). Reaction rates for segments ( 1 card required per
segment, all rates specified to base e, at. 20 oC). CALIBRATED
RATES for the TRWQ Model are shown in Appendix II, any other
values will not replicate calibrated simulations (Now1in, 1987).

\begin{tabular}{|c|c|c|c|c|c|}
\hline 61 & $1-2$ & I2 & $-\cdot$ & Card type $=61$ & $\cdots$ \\
\hline 61 & $3-4$ & I2 & $\mathrm{N}$ & Segment number & -- \\
\hline 61 & 5 & $-\cdot$ & $-\cdot$ & leave blank & $-\cdot$ \\
\hline 61 & $6-9$ & $\mathrm{~F} 4.0$ & $\operatorname{KCR}(\mathrm{N})$ & CBOD instream decay rate & 1/day \\
\hline 61 & $10 \div 13$ & F4. 0 & $\mathrm{KC}(\mathrm{N})$ & CBOD oxidation rate & 1/day \\
\hline 61 & $14-29$ & & -- & leave blank & -- \\
\hline 61 & $30-33$ & F4.0 & $\operatorname{KORNR}(\mathrm{N})$ & Organic-N instream decay rate & $1 /$ day \\
\hline 61 & $34-37$ & F4.0 & KORNF $(\mathrm{N})$ & Organic- to ammonia-N hydrolysis rate & 1/day \\
\hline 61 & $38-41$ & F4. 0 & $\mathrm{KNH} 4 \mathrm{R}(\mathrm{N})$ & Ammonia-N instream decay rate & $1 /$ day \\
\hline 61 & $42-45$ & F4. 0 & $\mathrm{KNH} 4 \mathrm{~F}(\mathrm{~N})$ & Ammonia to nitrite oxidation rate & $1 /$ day \\
\hline 61 & $46-49$ & F4. 0 & $\mathrm{KNO} 2 \mathrm{R}(\mathrm{N})$ & Nitrite-N instream decay rate & $1 /$ day \\
\hline 61 & $50-53$ & F4. 0 & $\mathrm{KNO} 2 \mathrm{~F}(\mathrm{~N})$ & Nitrite to nitrate oxidation rate & $1 /$ day \\
\hline 61 & $54-57$ & $\mathrm{~F} 4.0$ & $\mathrm{KNO} 3 \mathrm{R}(\mathrm{N})$ & Nitrate- $N$ instream decay rate & $1 /$ day \\
\hline 61 & $58-61$ & -- & $-\cdot$ & leave blank & -- \\
\hline 61 & $62-66$ & F5.0 & $\mathrm{KPO} 4 \mathrm{~B}(\mathrm{~N})$ & Orthophosphorus-A instream decay rate & 1/day \\
\hline 61 & $67-71$ & $-\cdot$ & -- & leave blank & -- \\
\hline 61 & $72-75$ & F4. 0 & $\operatorname{KNCR} 1(\mathrm{~N})$ & Orthophosphorus-B instream decay rate & 1/day \\
\hline 61 & $76-79$ & F4.0 & KNCR2 (N) & Phosphorus instream decay rate & 1/day \\
\hline
\end{tabular}


TABLE 1.--Specifications for input data--Continued.

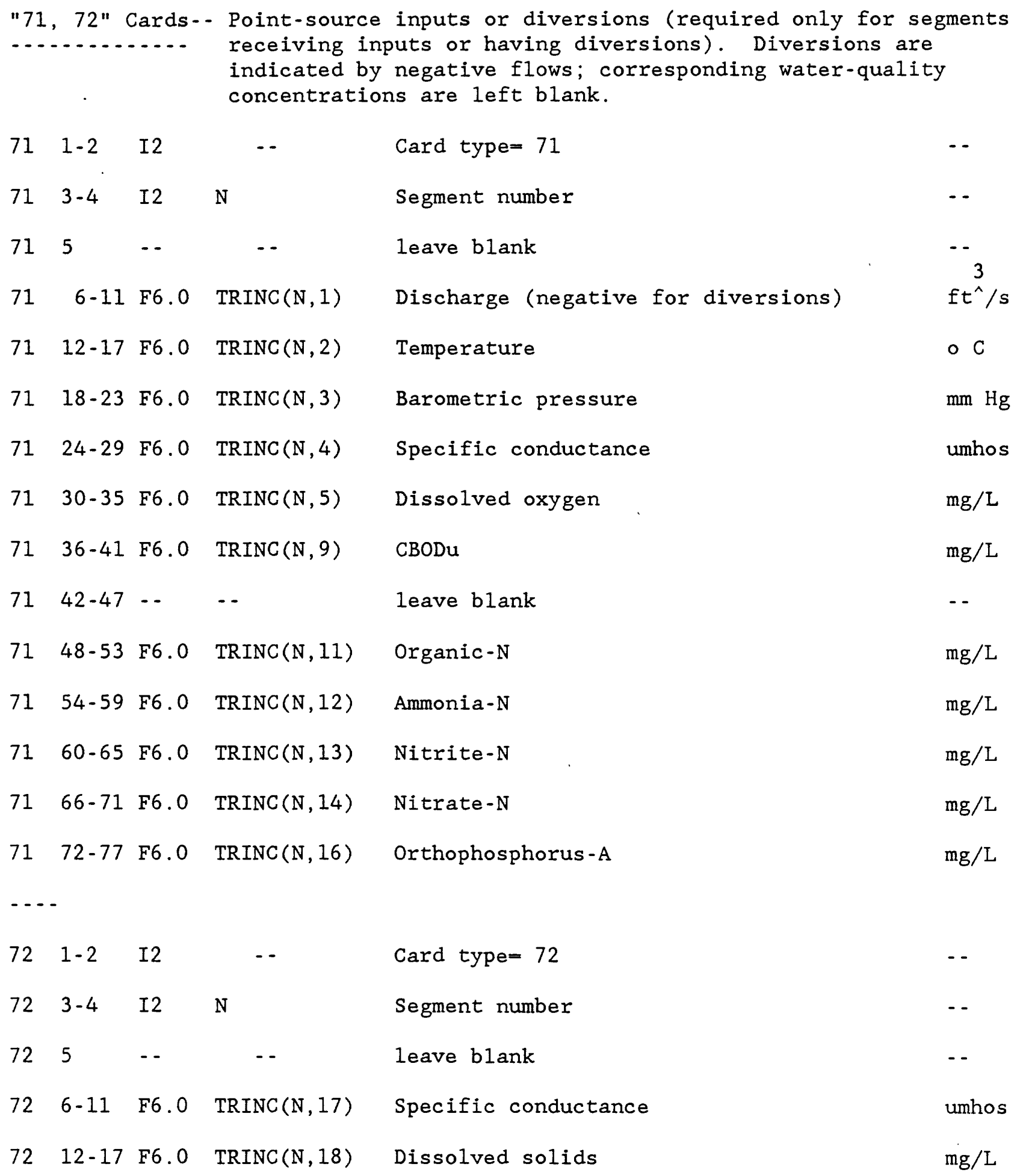


TABLE 1.--Specifications for input data--Continued.

$72 \quad 18-23-\cdots+-$

72 24-29 F6.0 TRINC (N, 20) Orthophosphorus-B

72 30-35 F6.0 TRINC $(N, 21)$ Phosphorus (tota1)

The following variables offer the option to specify inputs as loads. If coded, concentrations will be calculated from discharge and load. This option was not used for the calibrated TRWQ model.

$72 \quad 36-41$ F6.0 TRINL $(N, 9) \quad$ CBODu load

$7242-47$ - $\quad$ - $\quad$ leave blank

72 48-53 F6.0 TRINL(N, 11) Organic-N load

72 54-59 F6.0 TRINL(N,12) Ammonia-N load

72 60-65 F6.0 TRINL $(\mathrm{N}, 13) \quad$ Nitrite-N load

$7266-71$ F6.0 TRINL $(N, 14) \quad$ Nitrate-N load

$7272-77$ F6.0 TRINL(N,16) Orthophosphorus-A load
$1 b /$ day

$1 b /$ day

$1 b /$ day

$1 b /$ day

$1 b /$ day

$1 b /$ day

"81, 82" Cards--Nonpoint surface inflows (code only for receiving segments).

.......... If concentrations are coded as negative numbers, those values will be used to calculate the surface return concentration as a percentage of the instream concentration at the point of diversion specified by the segment number coded in column 78-79 on the 81 card.

\begin{tabular}{|c|c|c|c|c|c|}
\hline 81 & $1-2$ & I2 & $\cdots$ & Card type $=81$ & -- \\
\hline 81 & $3-4$ & I2 & $\mathrm{N}$ & Segment number & - \\
\hline 81 & 5 & -- & $\cdots$ & leave blank & - \\
\hline 81 & $6-11$ & F6. 0 & $\operatorname{RLINC}(N, 1)$ & Discharge & $f t^{\wedge} / s$ \\
\hline 81 & $12-17$ & $\mathrm{~F} 6.0$ & $\operatorname{RLINC}(N, 2)$ & Water temperature & $\circ \mathrm{C}$ \\
\hline 81 & $18-23$ & $\mathrm{~F} 6.0$ & $\operatorname{RLINC}(N, 3)$ & Specific conductance & umhos \\
\hline 81 & $24-29$ & $\mathrm{~F} 6.0$ & $\operatorname{RLINC}(N, 5)$ & Dissolved oxygen & $\mathrm{mg} / \mathrm{L}$ \\
\hline 81 & $30-35$ & F6. 0 & $\operatorname{RLINC}(N, 9)$ & CBODu & $\mathrm{mg} / \mathrm{L}$ \\
\hline 81 & $36-41$ & -- & - & leave blank & -- \\
\hline
\end{tabular}


TABLE 1.--Specifications for input data--Continued.

\begin{tabular}{|c|c|c|c|c|c|}
\hline 81 & $42-47$ & F6. 0 & $\operatorname{RLINC}(N, 11)$ & Organic-N & $\mathrm{mg} / \mathrm{L}$ \\
\hline 81 & $48-53$ & F6. 0 & $\operatorname{RLINC}(N, 12)$ & Ammonia-N & $\mathrm{mg} / \mathrm{L}$ \\
\hline 81 & $54-59$ & $\mathrm{~F} 6.0$ & $\operatorname{RLINC}(N, 13)$ & Nitrite-N & $\mathrm{mg} / \mathrm{L}$ \\
\hline 81 & $60-65$ & F6. 0 & $\operatorname{RLINC}(N, 14)$ & Nitrate-N & $\mathrm{mg} / \mathrm{L}$ \\
\hline 81 & $66-71$ & $\mathrm{~F} 6.0$ & $\operatorname{RLINC}(N, 16)$ & Orthophosphorus - A & $\mathrm{mg} / \mathrm{L}$ \\
\hline 81 & $72-77$ & $\mathrm{~F} 6.0$ & $\operatorname{RLINC}(N, 17)$ & Specific conductance & umhos \\
\hline 82 & $78-79$ & I2 & & $\begin{array}{l}\text { Segment number of originating diversion, } \\
\text { use if any surface return concentrations } \\
\text { are to be calculated as a percentage of } \\
\text { diverted concentrations. Code a } 0 \text { or } \\
\text { blank if returns originated from the same } \\
\text { segment as the return. }\end{array}$ & 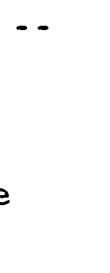 \\
\hline 82 & $1-2$ & I2 & - & Card type $=82$ & -- \\
\hline 82 & $3-4$ & $\mathrm{I} 2$ & -- & Segment number & -- \\
\hline 82 & 5 & - & $\cdots$ & leave blank & -- \\
\hline 82 & $6-11$ & F6. 0 & $\operatorname{RLINC}(N, 18)$ & Dissolved solids & $\mathrm{mg} / \mathrm{L}$ \\
\hline 82 & $12-17$ & - & $-\cdot$ & Leave blank & -- \\
\hline 82 & $18-23$ & F6. 0 & $\operatorname{RLINC}(N, 20)$ & Orthophosphorus - B & $\mathrm{mg} / \mathrm{L}$ \\
\hline 82 & $24-29$ & $\mathrm{~F} 6.0$ & $\operatorname{RLINC}(N, 21)$ & Phosphorus (total) & $\mathrm{mg} / \mathrm{L}$ \\
\hline
\end{tabular}

"83, 84" Cards--Quality of ground-water inflows (code only for receiving -.......... reaches.

$\begin{array}{lllclc}83 & 1-2 & \text { I2 } & \ldots & \text { Card type }=83 & \ldots \\ 83 & 3-4 & \text { I2 } & - & \text { Segment number } & \ldots \\ 83 & 5 & - & \ldots & \text { leave blank } & \ldots \\ 83 & 6-11 & F 6.0 & \operatorname{GLINC}(\mathrm{N}, 1) & \text { Discharge } & 3 \\ 83 & 12-17 & \mathrm{~F} 6.0 & \operatorname{GLINC}(\mathrm{N}, 2) & \text { Water temperature } & \mathrm{ft} / \mathrm{s} \\ 83 & 18-23 & \mathrm{~F} 6.0 & \operatorname{GLINC}(\mathrm{N}, 4) & \text { Specific conductance } & \text { umhos }\end{array}$


TABLE 1.--Specifications for input data--Continued.

\begin{tabular}{|c|c|c|c|c|c|}
\hline 83 & $24-29$ & F6. 0 & $\operatorname{GLINC}(N, 5)$ & Dissolved oxygen & $\mathrm{mg} / \mathrm{L}$ \\
\hline 83 & $30-35$ & F6. 0 & $\operatorname{GLINC}(\mathrm{N}, 9)$ & CBODu & $\mathrm{mg} / \mathrm{L}$ \\
\hline 83 & $36-41$ & -- & -- & leave blank & -- \\
\hline 83 & $42-47$ & F6. 0 & $\operatorname{GLINC}(N, 11)$ & Organic-N & $\mathrm{mg} / \mathrm{L}$ \\
\hline 83 & $48-53$ & F6. 0 & $\operatorname{GLINC}(\mathrm{N}, 12)$ & Ammonia-N & $\mathrm{mg} / \mathrm{L}$ \\
\hline 83 & $54-59$ & F6. 0 & $\operatorname{GLINC}(N, 13)$ & Nitrite-N & $\mathrm{mg} / \mathrm{L}$ \\
\hline 83 & $60-65$ & F6. 0 & $\operatorname{GLINC}(N, 14)$ & Nitrate $-\mathrm{N}$ & $\mathrm{mg} / \mathrm{L}$ \\
\hline 83 & $66-71$ & F6. 0 & $\operatorname{GLINC}(N, 16)$ & Orthophosphorus -A & $\mathrm{mg} / \mathrm{L}$ \\
\hline 83 & $72-77$ & F6. 0 & $\operatorname{GLINC}(N, 17)$ & Specific conductance & umhos \\
\hline 84 & $1-2$ & I2 & -- & Card type $=84$ & -- \\
\hline 84 & $3-4$ & $\mathrm{I} 2$ & -- & Segment number & -- \\
\hline 84 & 5 & -- & -- & leave blank & -- \\
\hline 84 & $6-11$ & F6. 0 & $\operatorname{GLINC}(\mathrm{N}, 18)$ & Dissolved solids & $\mathrm{mg} / \mathrm{L}$ \\
\hline 84 & $12-17$ & -- & -- & leave blank & -- \\
\hline 84 & $18-23$ & F6. 0 & $\operatorname{GLINC}(N, 20)$ & $\begin{array}{l}\text { Orthophoshorus - B } \\
\text { (see Appendix II) }\end{array}$ & $\mathrm{mg} / \mathrm{L}$ \\
\hline 84 & $24-29$ & F6. 0 & $\operatorname{GLINC}(N, 21)$ & $\begin{array}{l}\text { Phosphorus (total) } \\
\text { (see Appendix II) }\end{array}$ & $\mathrm{mg} / \mathrm{L}$ \\
\hline " - " " & Cards & $\begin{array}{r}- \\
\text { Obs } \\
\text { tab }\end{array}$ & $\begin{array}{l}\text { erved water } q \\
\text { ulated and pl }\end{array}$ & $\begin{array}{l}\text { ality (optional): values coded will be } \\
\text { ted with results of simulations. }\end{array}$ & \\
\hline 91 & $1-2$ & I2 & -- & Card type $=91$ & -- \\
\hline 91 & $6-9$ & F7. 0 & OBSMI (N) & River mile of sampling point & $\mathrm{mi}$ \\
\hline 91 & $10-16$ & F7. 0 & OBS $(N, 5)$ & Dissolved oxygen & $\mathrm{mg} / \mathrm{L}$ \\
\hline 91 & $17-23$ & F7. 0 & $\mathrm{OBS}(\mathrm{N}, 7)$ & DO percent saturation & 8 \\
\hline 91 & $24-30$ & F7. 0 & $\mathrm{OBS}(\mathrm{N}, 9)$ & CBODu & $\mathrm{mg} / \mathrm{L}$ \\
\hline 91 & $31-37$ & - - & - & leave blank & -- \\
\hline
\end{tabular}


TABLE 1.--Specifications for input data--Continued.

\begin{tabular}{|c|c|c|c|c|c|}
\hline 91 & $38-44$ & F7. 0 & OBS $(\mathrm{N}, 11)$ & Organic-N & $\mathrm{mg} / \mathrm{L}$ \\
\hline 91 & $45-51$ & F7. 0 & OBS $(N, 12)$ & Ammonia-N & $\mathrm{mg} / \mathrm{L}$ \\
\hline 91 & $52-58$ & F7. 0 & $\operatorname{OBS}(\mathrm{N}, 13)$ & Nitrite $-\mathrm{N}$ & $\mathrm{mg} / \mathrm{L}$ \\
\hline 91 & $59-65$ & F7. 0 & $\operatorname{OBS}(N, 14)$ & Nitrate $-\mathrm{N}$ & $\mathrm{mg} / \mathrm{L}$ \\
\hline 91 & $66-72$ & F7. 0 & $\mathrm{OBS}(\mathrm{N}, 15)$ & Orthophosphorus - A & $\mathrm{mg} / \mathrm{L}$ \\
\hline 91 & $73-79$ & F7. 0 & $\operatorname{OBS}(\mathrm{N}, 16)$ & Specific conductance & umhos \\
\hline 92 & $1-2$ & I2 & - & Card type $=92$ & -- \\
\hline 92 & $3-9$ & F7. 0 & $\operatorname{OBS}(\mathrm{N}, 17)$ & Dissolved solids & $\mathrm{mg} / \mathrm{L}$ \\
\hline 92 & $10-16$ & F7. 0 & $\operatorname{OBS}(\mathrm{N}, 18)$ & $\begin{array}{l}\text { Ratio of inorganic (ammonia + nitrite } \\
\quad+\text { nitrate) nitrogen to orthophosphorus }\end{array}$ & $\begin{array}{c}\text { moles/ } \\
\text { mole }\end{array}$ \\
\hline 92 & $17-23$ & F7. 0 & OBS $(N, 19)$ & Orthophosphorus - B & $\mathrm{mg} / \mathrm{L}$ \\
\hline 92 & $24-30$ & F7. 0 & $\operatorname{OBS}(\mathrm{N}, 20)$ & Phosphorus (total) & $\begin{array}{c}\mathrm{mg} / \mathrm{L} \\
3\end{array}$ \\
\hline 92 & $31-37$ & F7. 0 & $\operatorname{OBS}(\mathrm{N}, 21)$ & Discharge & $f t^{\wedge} / \mathrm{s}$ \\
\hline 92 & $38-44$ & F7. 0 & OBUNH3 $(\mathrm{N})$ & Un-ionized ammonia-N & $\mathrm{mg} / \mathrm{L}$ \\
\hline
\end{tabular}


APPENDIX I--LISTING OF COMPUTER CODE.

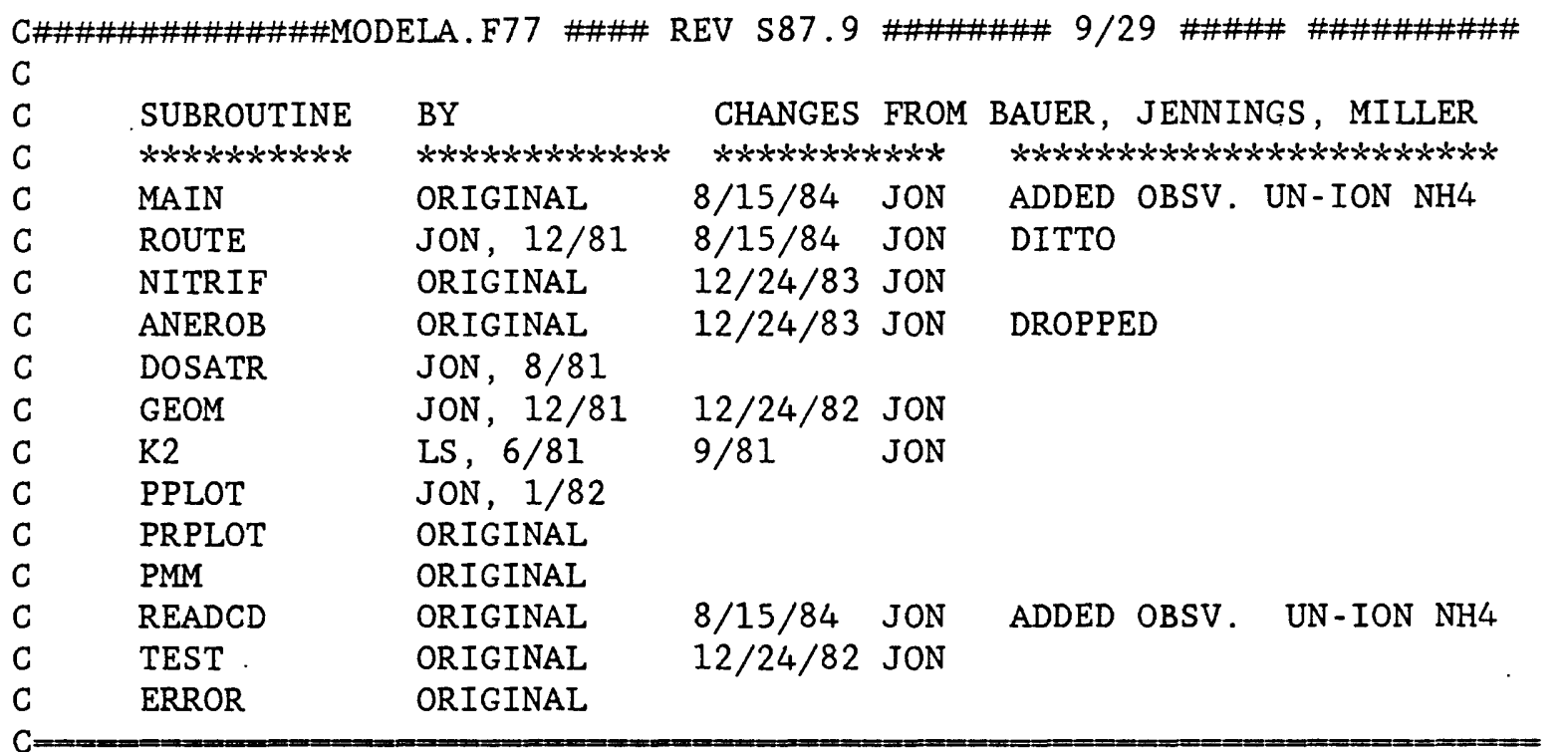

C LITERATURE CITATIONS FOR ALGRITHMS GIVEN IN BRACKETS [--.]

C

C UPDATES:

C $11 / 15 / 84$

C $* x * x * x$

C $* * * * * * x$

REVISED DATA READS SO CARDS WITH ILLEGITIMATE CARD NUMBERS MAY BE READ BUT WILL NOT STOP PROCESSING

C 1/17/85 CORRECTED READ ERROR FOR NON-STANDARD ESCAPE COEFFICIENTS

C $1 / 23 / 85$ FIXED ERROR IN K2 COMPUTATIONS: WAS TEMP CORRECTING IN ROUTE C $* x * * * x$

C $2 / 18 / 87$ CORRECTED LINE-PRINTER PLOT ROUTINES FOR CURRENT PRIMOS F77

C $* * * * * *$

C 5/87 REMOVED ALL OPTIONS NOT USED IN [NOWLIN, 1987]

C

C - - -

C $* * * * * *$ VARIABLES COMMON TO ONE OR MORE OF MAIN, READCD, ROUTE, NITRIF,

C $* * * * * * *$

C $* * * * * *$ SCALARS:

$\mathrm{C} * x * x * x$ SUBROUTINES

COMMON /VALL/ BDN, BNT, CARD, DELNH4, DELNO2, DELTT, DXPNT, EA,

1 ER, ICDBUF, IEND, IGRAPH, ITYPE, ILIN, IMODEL, INITSW, IPLOT,

2 IPO4, IPRMIN, IPNET, IRET, ISW1, ISWBAD, ITAB1, ITAB2, ITEST,

3 ITFLAG, IXGEOM, J, JJ, JSEG, KPLOT, K2T, KCT, KCRT, KORNRT,

4 KORNFT, KNH4RT, KNH4FT, KNO2RT, KNO2FT, KNO3RT, KP04BT, KNCR1T,

5 KNCR2T, LNCNTR, MPT, NCONSV, NNCONS, NSEG, PRINT, QTOT, TT,

C $* * x * * * x$

6 TTSUM, XEND, XL, XSTRT, IRESP, DATE

C $* * * * * *$ ARRAYS :

$C * * * * * *$

COMMON /ALL/ $\operatorname{AA}(50), \operatorname{AB}(50), \operatorname{ATRIBC}(50,21), \operatorname{ATRIBL}(50,21), \operatorname{BN}(50)$,

$1 \mathrm{BP}(50), \operatorname{BSEG}(50), \operatorname{CLONG}(5,10), \operatorname{CSHORT}(5,2), \operatorname{CUNIT}(5,2), \operatorname{DA}(50)$,

$2 \operatorname{DB}(50), \operatorname{DOSAT}(50), \operatorname{ENDC}(50,21), \operatorname{ENDL}(50,21), \operatorname{GEOM}(50,5)$,

$3 \operatorname{GLINC}(50,21), \operatorname{GLINL}(50,21), \operatorname{IGEOM}(50), \operatorname{IK} 2(50), \operatorname{K2}(50), \operatorname{KC}(50)$,

$4 \operatorname{KCR}(50), \operatorname{KORNR}(50), \operatorname{KORNF}(50), \operatorname{KNH} 4 R(50), \operatorname{KNH} 4 F(50), \operatorname{KNO} 2 R(50)$,

MAIN. .

MAIN. .

MAIN. .

MAIN. .

MAIN. .

MAIN. .

MAIN. .

MAIN. .

MAIN. .

MAIN. .

MAIN. .

MAIN. .

MAIN. .

MAIN. .

MAIN. .

MAIN. .

MAIN. .

MAIN. .

MAIN. .

MAIN. .

MAIN. .

MAIN. .

MAIN. .

MAIN. .

MAIN. .

MAIN. .

MAIN. .

MAIN. .

MAIN. .

MAIN. .

MAIN. .

COMMON

COMMON

COMMON

COMMON

COMMON

COMMON

COMMON

COMMON

COMMON

COMMON

COMMON

COMMON

COMMON

COMMON

COMMON

COMMON

COMMON

COMMON

COMMON

COMMON 
APPENDIX I--LISTING OF COMPUTER CODE--Continued.

$5 \mathrm{KNO} 2 \mathrm{~F}(50), \operatorname{KNO} 3 \mathrm{R}(50), \operatorname{KPO} 4 \mathrm{~B}(50), \operatorname{KNCR} 1(50), \operatorname{KNCR} 2(50)$,

$7 \operatorname{NDIV}(50), \operatorname{OBSC}(200,21), \operatorname{OBSMI}(200), \operatorname{OBUNH} 3(200), \operatorname{PGEOM}(6000,5)$,

$8 \operatorname{PH}(50), \operatorname{PLOTC}(6000,30), \operatorname{PLOTL}(6000,30), \operatorname{PNET}(50), \operatorname{PRCOEF}(50)$,

$9 \operatorname{RESP}(50), \operatorname{RLINC}(50,21), \operatorname{RLINL}(50,21), \operatorname{SCOND}(50), \operatorname{SDESC}(50,11)$,

\& $\operatorname{SFPM}(50), \operatorname{SLOPE}(50), \operatorname{SNAME}(50,6), \operatorname{STARTC}(21), \operatorname{STARTL}(21)$,

\& TCONC(21), TEMP(50), TITLE1(19), TITLE2(19), TLOAD(21),

$\& \operatorname{TRINC}(50,21), \operatorname{TRINL}(50,21), \operatorname{TA}(50), \operatorname{TB}(50), \operatorname{TTRIBC}(50,21)$,

\& TTRIBL $(50,21), \operatorname{UNH} 3(6000), \mathrm{VA}(50), \mathrm{VB}(50), \mathrm{WA}(50), \mathrm{WB}(50)$,

$\& \operatorname{XDIST}(6000), \operatorname{XLEN}(50), \operatorname{XSEG}(50), \operatorname{CTSVIG}(50)$

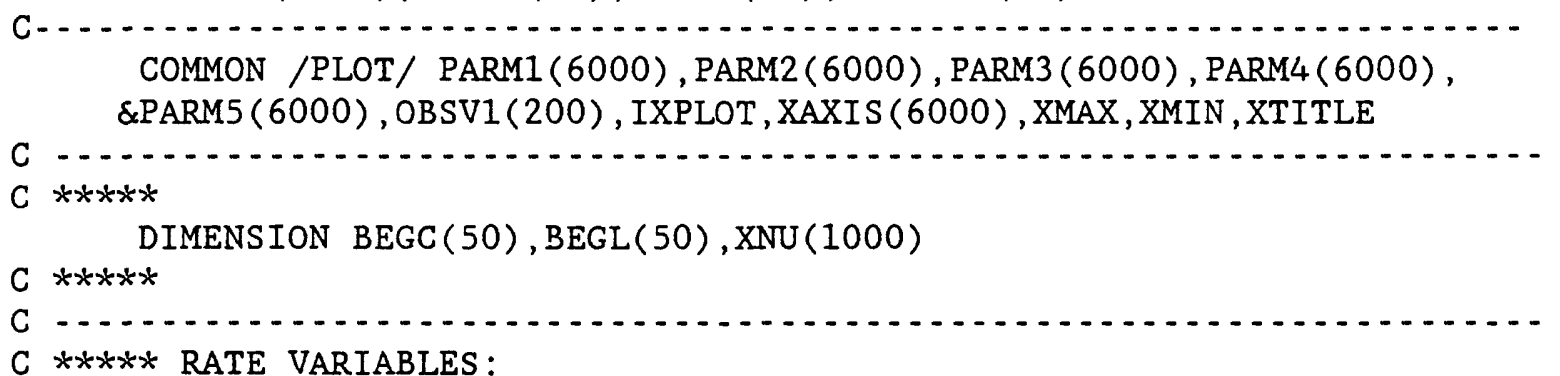

C ****** RATE VARIABLES:

REAL K2T, KCT , KCRT, KORNRT, KORNFT, KNH4RT, KNH4FT, KNO2RT, KNO2FT,

$\mathrm{C} * * * * * * *$

\& KNO3RT, KPO4BT, KNCR1T, KNCR2T, K2TRM

C ****** RATE ARRAYS:

C ********

REAL K2, KCR , KC, KNR , KN , KORNR, KORNF, KNH4R, KNH4F, KNO2R, KNO2F, KNO3R, $\& \mathrm{KPO4B}, \mathrm{KNCR} 1, \mathrm{KNCR} 2$

C....

INTEGER*4 PTITLE(15), YTITLE(9)

INTEGER $* 4$ CUNIT, CSHORT, CLONG

INTEGER *2 STAR, B2, FGEOM(50)

INTEGER 2 XTITLE(11), XTIME(11), XMILES (11)

INTEGER CARD, PRINT

CHARACTER $* 16$ DATE

REAL NTRM

C $* * * * x * *$

DATA STAR $/ 2 \mathrm{H} * * /$

$\begin{array}{lll}\text { DATA } & \text { B } 2 / 2 \mathrm{H} / \\ \text { DATA } & \text { B } 4 / \%\end{array}$

C

DATA B4 $/ "$ ' $/$

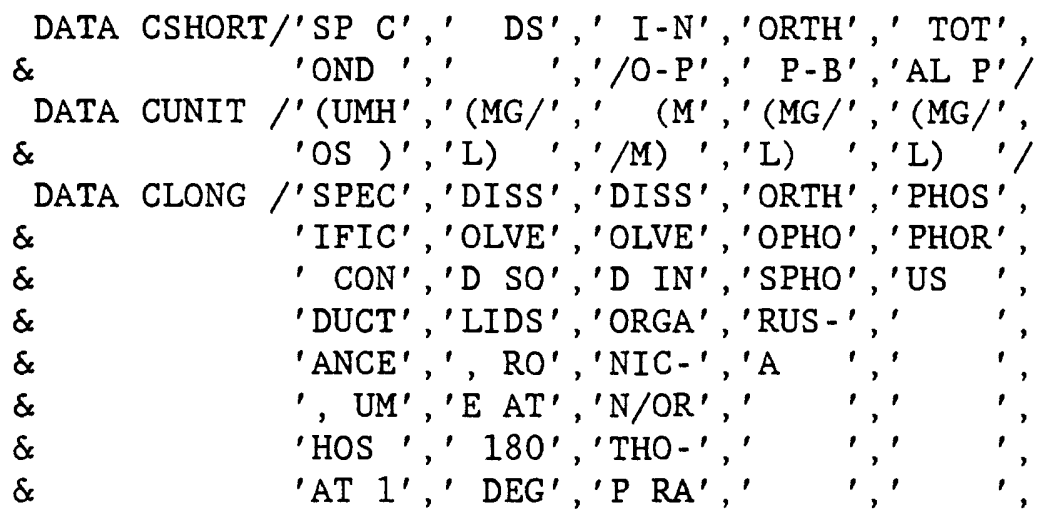

COMMON

COMMON

COMMON

COMMON

COMMON

COMMON

COMMON

COMMON

COMMON

COMMON

COMMON

COMMON

COMMON

MAIN. .

MAIN . .

MAIN. .

MAIN. .

MAIN . .

MAIN. .

MAIN . .

MAIN. .

MAIN. .

MAIN. .

MAIN. .

MAIN.

MAIN. .

MAIN. .

MAIN. .

MAIN. .

MAIN. .

MAIN. .

MAIN. .

MAIN.

MAIN. .

MAIN. .

MAIN. .

MAIN. .

MAIN. .

MAIN. .

MAIN. .

MAIN. .

MAIN. .

MAIN. .

MAIN. .

MAIN. .

MAIN. .

MAIN. .

MAIN. .

MAIN. .

MAIN. . 
APPENDIX I--LISTING OF COMPUTER CODE--Continued.

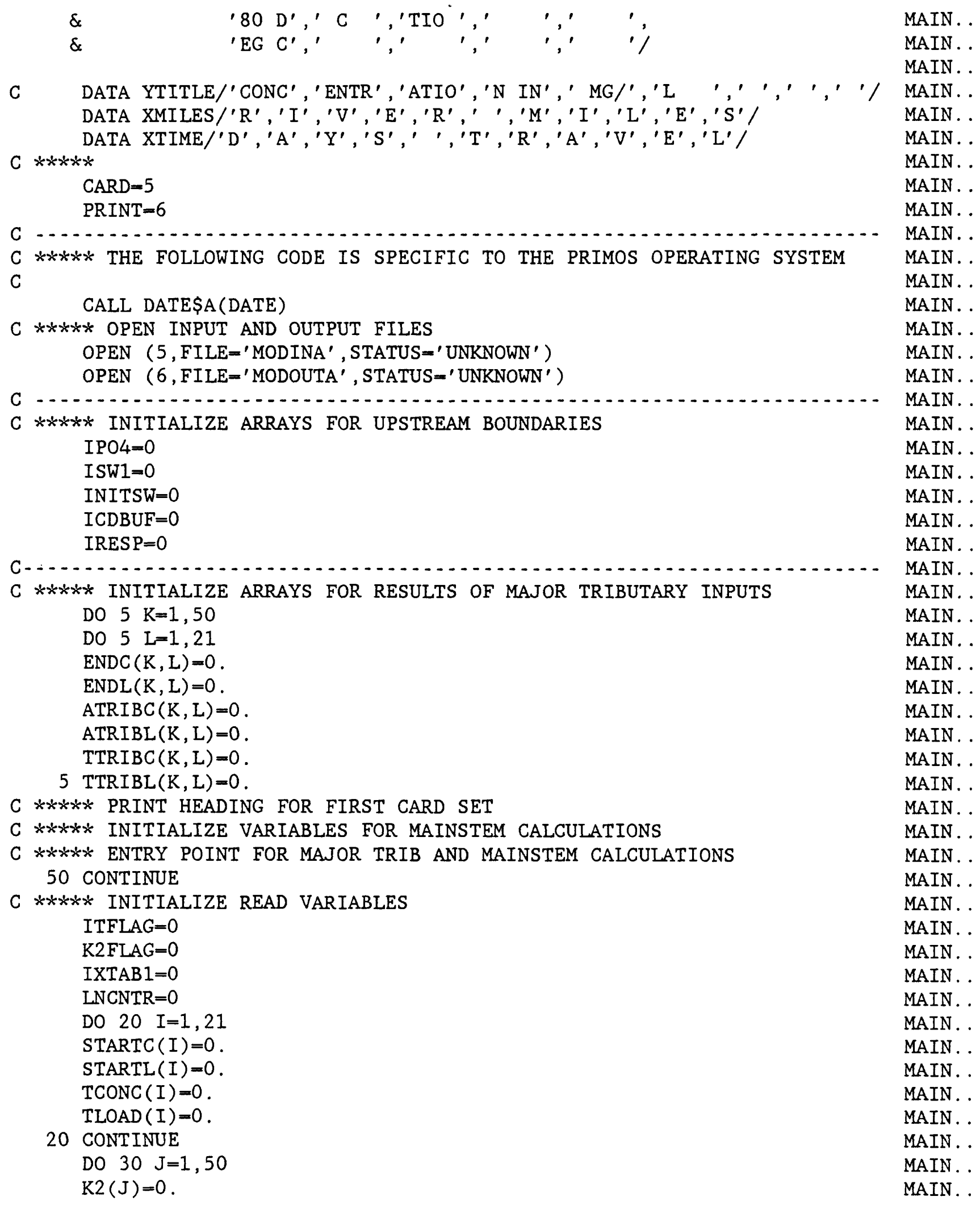


$\operatorname{CTSVIG}(J)=0$.

$\operatorname{KCR}(J)=0$.

$\mathrm{KC}(\mathrm{J})=0$.

$\operatorname{KORNF}(\mathrm{J})=0$.

$\operatorname{KORNR}(\mathrm{J})=0$.

$\mathrm{KNH} 4 \mathrm{~F}(\mathrm{~J})=0$.

$\mathrm{KNH} 4 \mathrm{R}(\mathrm{J})=0$.

$\mathrm{KNO} 2 \mathrm{~F}(\mathrm{~J})=0$.

$\mathrm{KNO} 2 \mathrm{R}(\mathrm{J})=0$.

$\operatorname{KNO3R}(\mathrm{J})=0$.

$\mathrm{KPO} 4 \mathrm{~B}(\mathrm{~J})=0$.

$\operatorname{KNCR} 1(\mathrm{~J})=0$.

$\operatorname{KNCR2}(\mathrm{J})=0$.

$\operatorname{SLOPE}(\mathrm{J})=0$.

$\operatorname{TEMP}(\mathrm{J})=0$.

$\mathrm{BP}(\mathrm{J})=0$.

$\operatorname{SCOND}(\mathrm{J})=0$.

$\mathrm{BN}(\mathrm{J})=0$.

$\operatorname{PNET}(\mathrm{J})=0$.

$\operatorname{RESP}(J)=0$.

$\operatorname{IK} 2(\mathrm{~J})=0$.

$\operatorname{DOSAT}(\mathrm{J})=0$.

$\operatorname{VA}(\mathrm{J})=0$.

$\mathrm{VB}(\mathrm{J})=0$.

$\mathrm{DA}(\mathrm{J})=0$.

$\mathrm{DB}(\mathrm{J})=0$.

$W A(J)=0$.

$\mathrm{WB}(\mathrm{J})=0$.

$A A(J)=0$.

$A B(J)=0$.

$\operatorname{BSEG}(\mathrm{J})=0$.

$\operatorname{XSEG}(J)=0$.

$\operatorname{IGEOM}(\mathrm{J})=0$.

$\mathrm{TA}(\mathrm{J})=0$.

$\mathrm{TB}(\mathrm{J})=0$.

$\operatorname{XLEN}(\mathrm{J})=0$.

DO $30 \mathrm{~K}=1,21$

$\operatorname{TRINC}(\mathrm{J}, \mathrm{K})=0$.

$\operatorname{TRINL}(\mathrm{J}, \mathrm{K})=0$.

$\operatorname{RLINC}(\mathrm{J}, \mathrm{K})=0$.

$\operatorname{RLINL}(\mathrm{J}, \mathrm{K})=0$.

$\operatorname{GLINC}(J, K)=0$.

$\operatorname{GLINL}(J, K)=0$.

$\operatorname{IGEOM}(\mathrm{J})=0$.

$\mathrm{PH}(\mathrm{J})=0$.

30 CONTINUE

DO $40 \mathrm{I}=1,6000$

DO $40 \mathrm{~J}=1,30$

DO $40 \mathrm{~K}=1,5$

$\operatorname{PGEOM}(I, K)=0$.

MAIN. .

MAIN. .

MAIN. .

MAIN. .

MAIN. .

MAIN. .

MAIN. .

MAIN. .

MAIN. .

MAIN. .

MAIN. .

MAIN. .

MAIN. .

MAIN. .

MAIN. .

MAIN. .

MAIN. .

MAIN. .

MAIN. .

MAIN. .

MAIN .

MAIN. .

MAIN. .

MAIN. .

MAIN. .

MAIN . .

MAIN. .

MAIN. .

MAIN. .

MAIN. .

MAIN. .

MAIN. .

MAIN. .

MAIN. .

MAIN. .

MAIN. .

MAIN .

MAIN. .

MAIN. .

MAIN. .

MAIN. .

MAIN. .

MAIN. .

MAIN . .

MAIN. .

MAIN. .

MAIN. .

MAIN. .

MAIN. .

MAIN. . 
APPENDIX I--LISTING OF COMPUTER CODE--Continued.

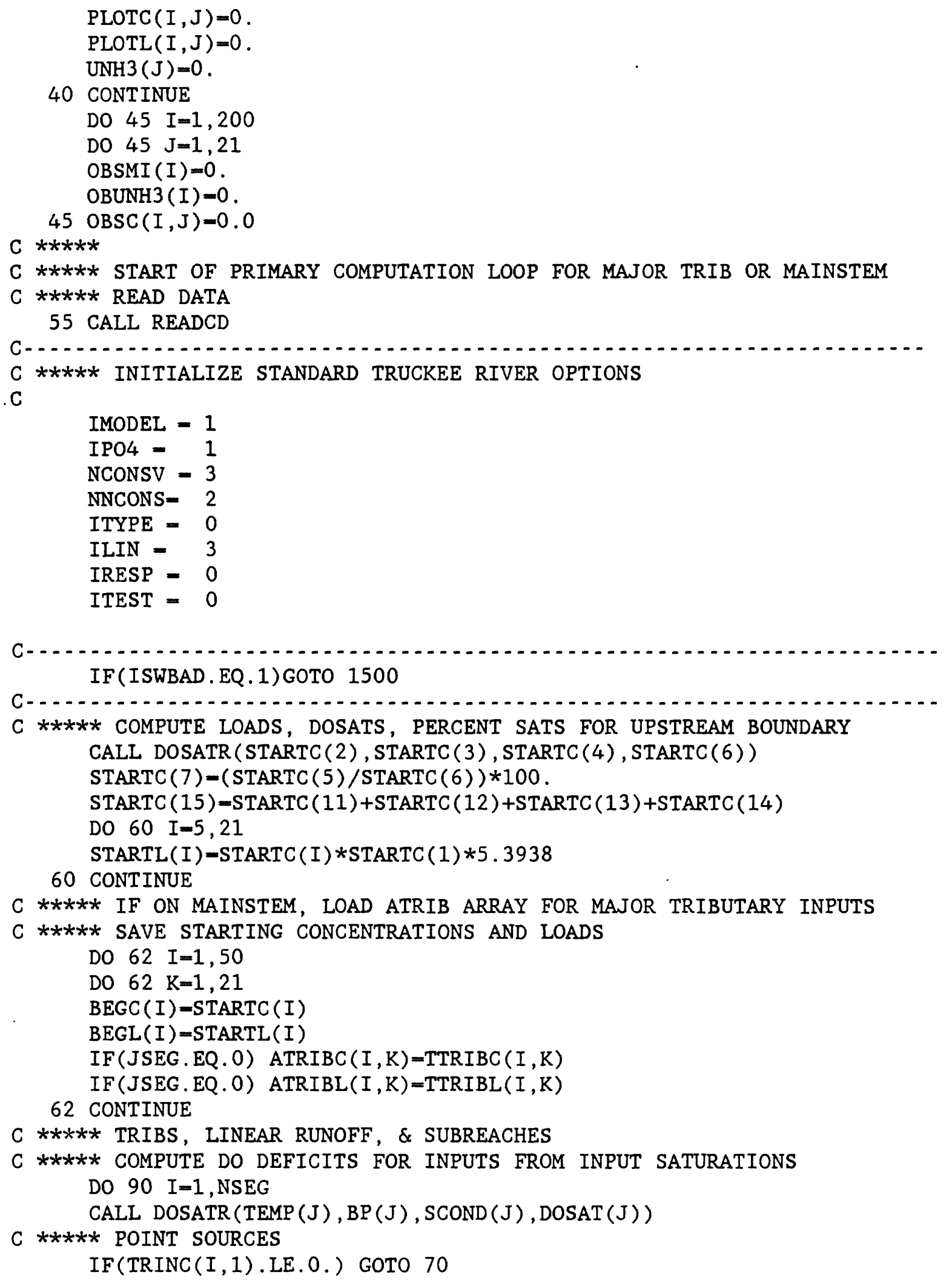

MAIN. .

MAIN. .

MAIN. .

MAIN. .

MAIN. .

MAIN. .

MAIN. .

MAIN. .

MAIN. .

MAIN. .

MAIN. .

MAIN .

MAIN. .

MAIN. .

MAIN. .

MAIN . .

MAIN . .

MAIN .

MAIN. .

MAIN . .

MAIN. .

MAIN. .

MAIN. .

MAIN. .

MAIN. .

MAIN. .

MAIN. .

MAIN. .

MAIN. .

MAIN. .

MAIN. .

MAIN. .

MAIN. .

MAIN. .

MAIN. .

MAIN. .

MAINN. .

MAIN. .

MAIN. .

MAIN. .

MAIN .

MAIN. .

MAIN. .

MAIN. .

MAIN. .

MAIN. .

MAIN. .

MAIN. .

MAIN. .

MAIN. . 
APPENDIX I--LISTING OF COMPUTER CODE--Continued.

CALL DOSATR(TRINC (I, 2), $\operatorname{TRINC}(I, 3), \operatorname{TRINC}(I, 4), \operatorname{TRINC}(I, 6))$

$\operatorname{TRINC}(I, 7)=(\operatorname{TRINC}(I, 5) / \operatorname{TRINC}(I, 6)) * 100$.

$\operatorname{TRINC}(I, 15)=\operatorname{TRINC}(I, 11)+\operatorname{TRINC}(I, 12)+\operatorname{TRINC}(I, 13)+\operatorname{TRINC}(I, 14)$

$\operatorname{TRINC}(I, 8)=\operatorname{TRINC}(I, 6)-\operatorname{TRINC}(I, 5)$

$T R I N L F=\operatorname{TRINC}(I, 1) * 5.3938$

TRINFI $=1$. /TRINLF

DO $65 \mathrm{~J}=5,21$

$\operatorname{TRINC}(I, J)=(\operatorname{TRINL}(I, J) * \operatorname{TRINFI})+\operatorname{TRINC}(I, J)$

$\operatorname{TRINL}(I, J)=0$.

$65 \operatorname{TRINL}(I, J)=\operatorname{TRINC}(I, J) * \operatorname{TRINLF}$

C ****** SURFACE NONPOINT RETURNS (MAY BE RECALCULATED IN 'ROUTE')

70 IF(RLINC (I, 1).LE.0.) GOTO 80

$\operatorname{IF}((\operatorname{RLINC}(I, 2)$.GE.0.) .AND. (RLINC $(I, 4)$.GE.0.))

\& CALL DOSATR(RLINC (I, 2), BP(I), RLINC $(I, 4), \operatorname{RLINC}(I, 6))$

$\operatorname{IF}(\operatorname{RLINC}(I, 6)$.GT .0.) $\operatorname{RLINC}(I, 7)=(\operatorname{RLINC}(I, 5) / \operatorname{RLINC}(I, 6)) * 100$.

$\operatorname{RLINC}(I, 15)=\operatorname{RLINC}(I, 11)+\operatorname{RLINC}(I, 12)+\operatorname{RLINC}(I, 13)+\operatorname{RLINC}(I, 14)$

$\operatorname{IF}(\operatorname{RLINC}(I, 6) . G T .0.) \operatorname{RLINC}(I, 8)=\operatorname{RLINC}(I, 6)-\operatorname{RLINC}(I, 5)$

DO $75 \mathrm{~J}=5,21$

IF(RLINC (I, J) .LT.0.) ITFLAG=1

$75 \operatorname{RLINL}(I, J)-R \operatorname{RINC}(I, J) * \operatorname{RLINC}(I, 1) * 5.3938$

C ***** GROUND-WATER NONPOINT RETURNS

$80 \operatorname{IF}(\operatorname{GLINC}(I, 1)$.LE.0.) GOTO 90

CALL DOSATR(GLINC $(I, 2), \operatorname{BP}(I), \operatorname{GLINC}(I, 4), \operatorname{GLINC}(I, 6))$

$\operatorname{GLINC}(I, 7)=(\operatorname{GLINC}(I, 5) / \operatorname{GLINC}(I, 6)) * 100$.

$\operatorname{GLINC}(I, 15)=\operatorname{GLINC}(I, 11)+\operatorname{GLINC}(I, 12)+\operatorname{GLINC}(I, 13)+\operatorname{GLINC}(I, 14)$

$\operatorname{IF}(\operatorname{GLINC}(I, 4) . G T .0.) \operatorname{GLINC}(I, 8)=\operatorname{GLINC}(I, 6)-\operatorname{GLINC}(I, 5)$

DO $85 \mathrm{~J}=5,21$

$85 \operatorname{GLINL}(I, J)=\operatorname{GLINC}(I, J) * \operatorname{GLINC}(I, 1) * 5.3938$

90 CONTINUE

$C * * * * *$ COMPUTE SUBREACH ENDS, XSEG(NSEG)

L-NSEG - 1

DO $100 \quad I=1, L$

$\operatorname{XSEG}(I)=\operatorname{BSEG}(I+1)$

100 CONTINUE

XSEG (NSEG) -XEND

DO $110 \mathrm{~J}=1$, NSEG

$\operatorname{XLEN}(\mathrm{J})=\operatorname{BSEG}(\mathrm{J})-\mathrm{XSEG}(\mathrm{J})$

110 CONTINUE

C ***** PRINT CONTENTS FOR EACH RUN'S OUTPUT

IF (ITABI.GT.0) GOTO 717

IF(IXTAB1.EQ.1) GOTO 360

WRITE (PRINT, 120) TITLE1, DATE, TITLE2

120 FORMAT (1H1,16X,'U.S. GEOLOGICAL SURVEY, WATER RESOURCES DIVISION'

$\&,^{\prime}:^{\prime}, 2 \mathrm{X},{ }^{\prime}$ TRUCKEE RIVER WATER-QUALITY MODEL', 15X, '[REV 87.9]',

$\& / 1 \mathrm{X}, 130\left(^{\prime}\right.$ - ' $\left.\left.^{\prime}\right), / 4 \mathrm{X}, / 20 \mathrm{X}, 19 \mathrm{~A} 4,10 \mathrm{X}, \mathrm{Al} 6, / 20 \mathrm{X}, 19 \mathrm{~A} 4\right)$

IF(JSEG.NE.0) WRITE(PRINT, 125) JSEG

125 FORMAT (/30X, 'RESULTS FOR MAJOR TRIBUTARY ENTERING SUBREACH ', I2, $\left.\&^{\prime} \cdot{ }^{\prime}, /\right)$

WRITE (PRINT, 130)

MAIN . .

MAIN. .

MAIN. .

MAIN . .

MAIN. .

MAIN. .

MAIN. .

MAIN. .

MAIN. .

MAIN. .

MAIN. .

MAIN. .

MAIN. .

MAIN. .

MAIN. .

MAIN. :

MAIN. .

MAIN . .

MAIN. .

MAIN. .

MAIN. .

MAIN. .

MAIN. .

MAIN. .

MAIN. .

MAIN. .

MAIN. .

MAIN. .

MAIN. .

MAIN. .

MAIN. .

MAIN. .

MAIN. .

MAIN. .

MAIN. .

MAIN. .

MAIN. .

MAIN. .

MAIN. .

MAIN. .

MAIN. .

MAIN. .

MAIN. .

MAIN. .

MAIN. .

MAIN. .

MAIN. .

MAIN. .

MAIN. .

MAIN. . 
APPENDIX I--LISTING OF COMPUTER CODE--Continued.

130 FORMAT (/30X, 'TABLE OF CONTENTS FOR OUTPUT FROM THIS MODEL RUN.' , $\& / 30 \mathrm{X}, 49\left({ }^{\prime}-^{\prime}\right), / / 10 \mathrm{X}, \mathrm{T}^{\left.\mathrm{T} A B L E S^{\prime}, / 10 \mathrm{X}, 6\left({ }^{\prime}-{ }^{\prime}\right)\right)}$

WRITE (PRINT, 140)

140 FORMAT (/11X,' 'TABLE 1.--MODEL OPTIONS FOR THIS RUN.',/17X,'2.--SUB \$REACH DESCRIPTIONS.',/17X,'3.--MEAN SUBREACH ENVIRONMENTAL FACTORS $\& .^{\prime}, / 17 \mathrm{X}, ' 4 .-$-SUMMARY OF TRIBUTARY AND POINT-SOURCE INPUTS.') WRITE (PRINT , 160)

160 FORMAT $(17 \mathrm{X}, ' 5 .--$ SUBREACH HYDRAULICS DATA.') WRITE (PRINT, 170)

170 FORMAT $(17 \mathrm{X}, ' 6$. - SUBREACH REACTION COEFFICIENTS .') IF (MPT.NE . 0)WRITE (PRINT, 180)

180 FORMAT $(17 \mathrm{X}, 17 \ldots$ - - OBSERVED WATER-QUALITY DATA.' $)$ WRITE (PRINT, 210)

210 FORMAT (17X,'8.--RESULTS OF COMPUTATIONS FOR DISSOLVED-OXYGEN ', \&'PARAMETERS.' $, / 17 \mathrm{X}, ' 9 .-$ RESULTS OF COMPUTATIONS FOR OTHER ', $\&$ 'CONSTITUENTS.' $)$ WRITE (PRINT , 215)

215 FORMAT (//10X,' FIGURES' , /10X, 7('-' $), /)$ WRITE (PRINT , 217)

217 FORMAT(/11X,' FIGURE 1.--RIVER PROFILE OF CALCULATED AND ' , \&'OBSERVED WATER DISCHARGE.' )

IF (NCONSV.NE.0)WRITE (PRINT, 220) (CSHORT (1,K), K=1, 2)

220 FORMAT (17X, '2A. - -RIVER PROFILE OF CALCULATED AND OBSERVED: ' , 2A4) IF (NCONSV.NE. 1)WRITE (PRINT, 230) (CSHORT $(2, K), K=1,2$ )

230 FORMAT (17X, ' 2B. - -RIVER PROFILE OF CALCULATED AND OBSERVED: ', 2A4) IF (NCONSV.EQ. 3)WRITE (PRINT, 240) (CSHORT (3,K), K=1,2)

240 FORMAT (17X,'2C.--RIVER PROFILE OF CALCULATED AND OBSERVED: ',2A4) WRITE (PRINT, 260)

260 FORMAT $(18 \mathrm{X}, ' 3 .-$-RIVER PROFILE OF CALCULATED AND OBSERVED DISSOLVED 1-OXYGEN CONCENTRATIONS.'/18X,'4.--RIVER PROFILE OF CALCULATED AND 2OBSERVED DISSOLVED-OXYGEN SATURATIONS.'/18X,'5.--RIVER PROFILE OF \&CALCULATED DISSOLVED-OXYGEN DEFICITS.',/18X,'6.--RIVER PROFILE OF \&CALCULATED AND OBSERVED CBODU CONCENTRATIONS.' ) IF (IMODEL. EQ. 1)WRITE (PRINT, 290)

290 FORMAT (18X,'8.--RIVER PROFILE OF CALCULATED AND OBSERVED ORGANIC 1NITROGEN CONCENTRATIONS.'/18X,'9.--RIVER PROFILE OF CALCULATED AND 2 OBSERVED AMMONIA-NITROGEN CONCENTRATION.'/17X,'10 - - RIVER PROFILE 3 OF CALCULATED AND OBSERVED NITRITE-NITROGEN CONCENTRATIONS .,$/ 17$ 4X,'11. - -RIVER PROFILE OF CALCULATED AND OBSERVED NITRATE-NITROGEN 5CONCENTRATIONS.'/17X,'12.--RIVER PROFILE OF CALCULATED AND OBSERVE 6D TOTAL NITROGEN CONCENTRATIONS.' )

IF (IPO4.EQ . 1)WRITE (PRINT, 310)

310 FORMAT (17X,'13. - -RIVER PROFILE OF CALCULATED AND OBSERVED PHOSPHA ITE-PHOSPHORUS CONCENTRATIONS.')

IF (NNCONS . NE . 0) WRITE (PRINT , 330) ((CSHORT ( I , J ) , J=1, 2) , I=4, 5)

330 FORMAT (17X,' 14.--RIVER PROFILE OF CALCULATED AND OBSERVED ', 2A4,' $\&$ CONCENTRATIONS.' $17 \mathrm{X}, ' 15 .-$-RIVER PROFILE OF CALCULATED AND OBSERV \$ED ', 2A4,' CONCENTRATIONS.')

WRITE (PRINT, 350)

350 FORMAT (17X,'16.--RIVER PROFILE OF CALCULATED REAERATION RATES (K2

MAIN. . MAIN. . MAIN. . MAIN. . MAIN. . MAIN. . MAIN. . MAIN. . MAIN. . MAIN. . MAIN. . MAIN. . MAIN. . MAIN. . MAIN. . MAIN. . MAIN. . MAIN. . MAIN. . MAIN. . MAIN. . MAIN. . MAIN. . MAIN. . MAIN. . MAIN. . MAIN. . MAIN. . MAIN. . MAIN. . MAIN. . MAIN. . MAIN. . MAIN. . MAIN. . MAIN. . MAIN. . MAIN. . MAIN. . MAIN. . MAIN. . MAIN. . MAIN. . MAIN. . MAIN. . MAIN. . MAIN. . MAIN. . MAIN. . MAIN. . 
APPENDIX I--LISTING OF COMPUTER CODE--Continued.

\&) $.^{\prime}, / 17 \mathrm{X}, ' 17,--\mathrm{RIV}^{\prime}$,

1'ER PROFILE OF MEAN VELOCITY.'/17X,'18.--RIVER PROFILE OF CUMULATI 2VE TRAVEL TIME.'/17X,'17.--RIVER PROFILE OF MEAN CROSS-SECTIONAL A 3REA.',/17X,'20.--RIVER PROFILE OF MEAN CHANNEL DEPTH.'/17X,'21.--R 4IVER PROFILE OF MEAN CHANNEL WIDTH.' ) IXTAB $1=1$

C ***** PRINT TABLE 1. MODEL OPTIONS AND UPSTREAM BOUNDARY CONDITIONS
360 WRITE (PRINT, 120) TITLE1, DATE, TITLE2
IF(JSEG.NE.0) WRITE(PRINT, 125) JSEG
WRITE (PRINT, 370)

370 FORMAT (/3OX,'TABLE 1.--MODEL OPTIONS AND UPSTREAM BOUNDARY CONDITI 1ONS.'/30X,57('-'),//15X, 'MODEL OPTIONS', 28X, 'SET THIS RUN',/15X,13 $2(1 \mathrm{H}=), 28 \mathrm{X}, 12(1 \mathrm{H}=))$

$C * * * * *$ CHANNEL GEOM ONLY WRITE (PRINT , 570) NSEG, XSTRT, XSEG(NSEG), DXPNT

570 FORMAT (/20X, 21HNUMBER OF SUBREACHES : 16X, I2 ,//20X, 20HSTARTING RIV 1ER MILE: ,14X,F8.2,/20X,18HENDING RIVER MILE:,16X,F8.2,//20X, ' CALCU 2LATION INTERVAL: ', 14X,F5.2,' MILE.') IF (ILIN.EQ.0) WRITE (PRINT , 400)

400 FORMAT (/2OX, 22HLINEAR RUNOFF MODELED: , 15X, 2HNO) IF (ILIN. EQ. 1)WRITE (PRINT , 420)

420 FORMAT (/20X, 22HLINEAR RUNOFF MODELED: ,15X,' SURFACE RETURNS.') IF (ILIN.EQ. 2) WRITE (PRINT, 421)

421 FORMAT (/20X,' LINEAR RUNOFF MODELED:' , 15X, 'GROUND-WATER RETURNS.' ) IF(ILIN.EQ. 3)WRITE (PRINT, 422)

422 FORMAT (/20X, 22HLINEAR RUNOFF MODELED: 15X, 'SURFACE AND GROUND-WAT \&ER RETURNS.' )

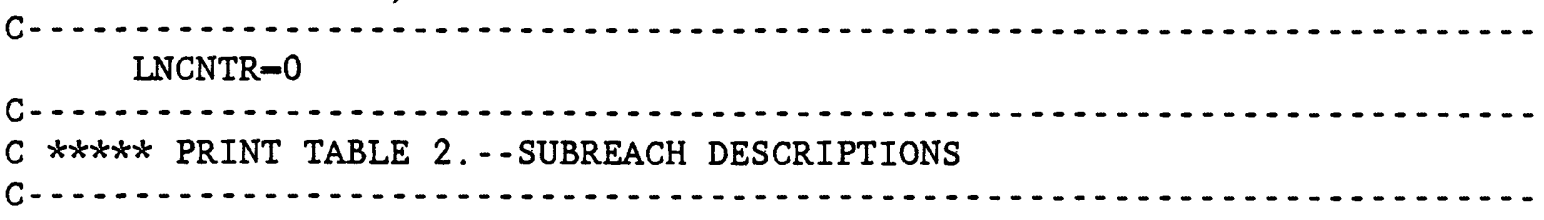

IF(JSEG.EQ.0) WRITE (PRINT, 120) TITLE1, DATE, TITLE2

WRITE (PRINT , 690)

690 FORMAT (/30X,' TABLE 2.--SUBREACH DESCRIPTIONS .' ,

$1 / 30 \mathrm{X}, 32('-1), / / 5 \mathrm{X}$, ' SUBREACH' , 11X, 'NAME' , 16X, 'RIVER MILES' , 9X,

2'TOTAL' , 3X, ' DESCRIPTION' , /43X, 'BEGIN' , 7X, 'END' , 6X, 'LENGTH' , /5X,

$\left.38\left({ }^{\prime}-^{\prime}\right), 2 \mathrm{X}, 26\left(^{\prime}-^{\prime}\right), 2 \mathrm{X}, 17\left(^{\prime}-^{\prime}\right), 2 \mathrm{X}, 8\left(^{\prime}-^{\prime}\right), 2 \mathrm{X}, 44\left(^{\prime}-^{\prime}\right) /\right)$

DO $700 \quad I=1$, NSEG

WRITE (PRINT, 710$) I,(\operatorname{SNAME}(I, L), \operatorname{L}=1,6), \operatorname{BSEG}(I), \operatorname{XSEG}(I), \operatorname{XLEN}(I)$,

$1(\operatorname{SDESC}(I, L), L=1,11)$

IF(XLEN(I).LE.0) WRITE(PRINT, 709) I

709 FORMAT( $1 \mathrm{X}$, 'SUBREACH ',I2,' LENGTH IS LESS THAN OR EQUAL TO 0, PROC \&ESSING STOPPED!' )

700 CONTINUE

710 FORMAT (7X, I2, 6X, 6A4, 3(2X, F8, 2), 5X, 11A4)

TOTAL=BSEG ( 1 ) -XSEG (NSEG)

WRITE (PRINT, 715) TOTAL

MAIN. .

MAIN.

MAIN.

MAIN.

MAIN. .

MAIN. .

MAIN. .

MAIN. .

MAIN. .

MAIN .

MAIN.

MAIN.

MAIN.

MAIN. .

MAIN. .

MAIN. .

MAIN. .

MAIN.

MAIN. .

MAIN. .

MAIN. .

MAIN. .

MAIN. .

MAIN. .

MAIN. .

MAIN.

MAIN.

MAIN. .

MAIN.

MAIN.

MAIN.

MAIN. .

MAIN. .

MAIN.

MAIN. .

MAIN. .

MAIN. .

MAIN.

MAIN.

MAIN.

MAIN.

MAIN. .

MAIN.

MAIN. .

MAIN. .

MAIN.

MAIN .

MAIN .

MAIN. .

MAIN. . 
APPENDIX I--LISTING OF COMPUTER CODE--Continued.

715 FORMAT ( $61 \mathrm{X}, 8\left({ }^{\prime}-^{\prime}\right), / 61 \mathrm{X}, \mathrm{F} 8.2,1 \mathrm{X}, \mathrm{e}^{\prime}$ MILES TOTAL LENGTH MODELED.')

MAIN. .

717 CONTINUE

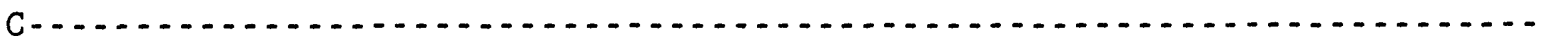

C ****** PRINT TABLE 3.--MEAN SUBREACH ENVIRONMENTAL FACTORS.

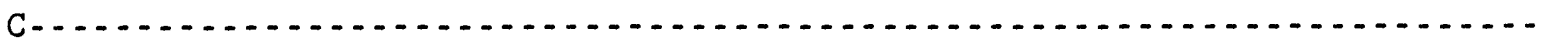

DO $720 \mathrm{~J}=1$, NSEG

$\operatorname{CALL} \operatorname{DOSATR}(\operatorname{TEMP}(\mathrm{J}), \operatorname{BP}(\mathrm{J}), \operatorname{SCOND}(\mathrm{J}), \operatorname{DOSAT}(\mathrm{J}))$

720 CONTINUE

IF(ITAB1.GT.3) GOTO 741

IF(JSEG.EQ. 0) WRITE (PRINT, 120) TITLE1, DATE, TITLE2

WRITE (PRINT , 730)

730 FORMAT (/30X,' TABLE 3.--MEAN SUBREACH ENVIRONMENTAL',

\&' FACTORS $.^{\prime}, / 30 \mathrm{X}, 46\left({ }^{\prime}-{ }^{\prime}\right), / / 34 \mathrm{X}$, ' DO SATURATION CONTROLS'

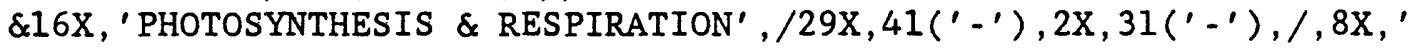

\&SUBREACH' , 14X, 'MEAN' , 2X, 'BAROMETRIC' , 2X, 'SPECIFIC' , 6X, ' DO AT' , 8X, '

\&NET' , 9X, 'NET' , 6X, 3X, 'BENTHIC' , 6X, 'MEAN' ,/30X, 'TEMP' ,

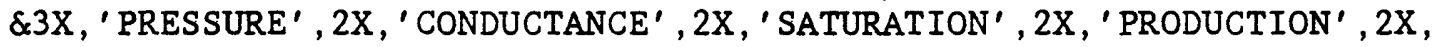

\&'RESPIRATION' , 2X, 4X, 'DEMAND' , 8X, 'PH' ,/29X, ' (DEG C)' ,

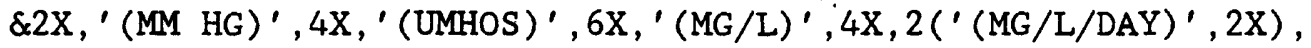

$\left.\& 3 \mathrm{X},{ }^{\prime}(\mathrm{GM} / \mathrm{SQ} \mathrm{M} / \mathrm{DAY})^{\prime} / 1 \mathrm{X}, 132\left(^{\prime}-^{\prime}\right)\right)$

WRITE (PRINT, 740) (J, ( $\operatorname{SNAME}(J, L), L=1,6), \operatorname{TEMP}(J), B P(J), \operatorname{SCOND}(J), D O S$

$\operatorname{1AT}(\mathrm{J}), \operatorname{PNET}(\mathrm{J}), \operatorname{RESP}(\mathrm{J}), \operatorname{BN}(\mathrm{J}), \operatorname{PH}(\mathrm{J}), \mathrm{J}=1, \mathrm{NSEG})$

740 FORMAT (1X, I2, 2X, 6A4, 2X, F4.1, 4X, F4.0, 6X, F6.0, 6X, F6.1, 5X, F6. 1, 5X, F6 $1.1,5 \mathrm{X}, 6 \mathrm{X}, \mathrm{F} 6.1,10 \mathrm{X}, \mathrm{F} 3.1$ )

741 IF ((ITAB1.NE.4).AND.(ITAB1.NE.0)) GOTO 750

MAIN. .

MAIN. .

MAIN. .

MAIN. .

MAIN. .

MAIN. .

MAIN. .

MAIN. .

MAIN. .

MAIN. .

MAIN. .

MAIN. .

MAIN. .

MAIN. .

MAIN. .

MAIN. .

MAIN. .

MAIN.

MAIN. .

MAIN. .

MAIN. .

MAIN. .

MAIN. .

MAIN. .

MAIN. .

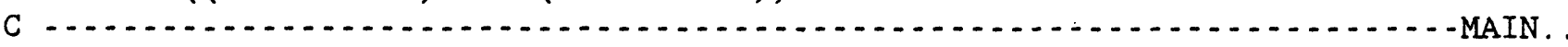

C $* * * * *$ PRINT TABLE 4A. - SUMMARY OF INPUTS TO SUBREACHES: CONCENTRATIONS. MAIN.

C- . . . . . . .

C ****** HEADING

WRITE (PRINT, 120) TITLE1, DATE, TITLE2

WRITE(PRINT , 820)

820 FORMAT (/3OX, 'TABLE 4A. --SUMMARY OF INPUTS TO SUBREACHES: ',

$\&$ 'CONCENTRATIONS.' $\left./ 30 \mathrm{X}, 60\left({ }^{\prime}-{ }^{\prime}\right)\right)$

WRITE (PRINT, 826)

826 FORMAT (/1OX,' (NOTE-- NEGATIVE CONCENTRATIONS TO BE RECOMPUTED AS'

$\&$,' A FUNCTION OF DIVERTED QUALITY \& WILL FOLLOW TABLE 8.)')

WRITE (PRINT, 830) ((CSHORT ( $L, N), N=1,2), L-1,2)$,

$\&((\operatorname{CSHORT}(\mathrm{L}, \mathrm{N}), \mathrm{N}=1,2), \mathrm{L}=4,5)$

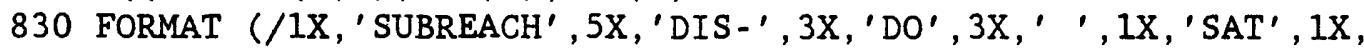

$\&$ 'DO DEF', $2 \mathrm{X},,^{\prime} \mathrm{CBODU}$ ' $, 2 \mathrm{X}, \mathrm{\prime}^{\prime} \mathrm{ORG}-\mathrm{N}^{\prime}, 2 \mathrm{X}$, 'NH4-N', $2 \mathrm{X}$, ' $\mathrm{NO} 2-\mathrm{N}^{\prime}$,

$\& 2 \mathrm{X},{ }^{\prime} \mathrm{N} 03-\mathrm{N}^{\prime}, 1 \mathrm{X},,^{\mathrm{T}} \mathrm{TOT}-\mathrm{N}^{\prime}, 1 \mathrm{X},{ }^{\prime} \mathrm{O}-\mathrm{P}-\mathrm{A}^{\prime}, 2 \mathrm{X}, 2 \mathrm{~A} 4,2 \mathrm{X}, 2 \mathrm{~A} 4,1 \mathrm{X}, 2 \mathrm{~A} 4,2 \mathrm{~A} 4,2 \mathrm{X}$,

$\&$ 'DIVERTED' $/ 13 \mathrm{X}$, 'CHARGE', $105 \mathrm{X}$, ' SUBR NO.' $\left., / 1 \mathrm{X}, 132\left({ }^{\prime}-'\right), /\right)$

$C * * * * *$ CONCENTRATIONS

WRITE (PRINT , 835) BEGC (1), BEGC (5), (BEGC (K), K=7,9),

$\&(B E G C(K), K=11,18),(B E G C(K), K=20,21)$

835 FORMAT (1X,' UPSTREAM:' ,F9.1,F6.1,F6.0,F6.1, 7F7.2 , 2F8.2 , 2F10.2/)

DO $850 \quad I=1$, NSEG

IF (ATRIBC(I, 1).GT. (0.0)) WRITE(PRINT, 839) I, ATRIBC (I, 1),

$\& \operatorname{ATRIBC}(I, 5),(\operatorname{ATRIBC}(I, K), K=7,9),(\operatorname{ATRIBC}(I, K), K=11,18)$,

MAIN. .

MAIN. .

MAIN. .

MAIN. .

MAIN. .

MAIN. .

MAIN. .

MAIN. .

MAIN. .

MAIN. .

MAIN. .

MAIN. .

MAIN. .

MAIN. .

MAIN. .

MAIN. .

MAIN. .

MAIN. .

MAIN. .

MAIN. .

MAIN. . $\&$ (ATRIBC $(I, K), K=20,21)$

MAIN. . 
APPENDIX I--LISTING OF COMPUTER CODE--Continued.

839 FORMAT (1X, I2 , 2X, 'MT' , 3X, F9.1, F6.1, F6.0, F6.1, 7F7.2, 2F8 . 2, 2F10.2)

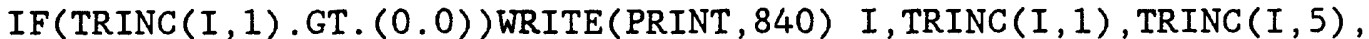
\& (TRINC $(I, K), K=7,9),(\operatorname{TRINC}(I, K), K=11,18),(\operatorname{TRINC}(I, K), K=20,21)$

840 FORMAT (1X, I2, 2X, 'PS' , 3X, F9.1, F6.1, F6.0, F6.1, 7F7.2, 2F8.2, 2F10.2) $\operatorname{IF}(\operatorname{TRINC}(I, 1)$. LT. (0.0))WRITE (PRINT, 841) I, TRINC ( $I, 1$ )

841 FORMAT (1X, I2, 2X, 'PS', 3X, F9.1)

IF (ILIN.EQ.0) GOTO 850

$\operatorname{IF}(\operatorname{RLINC}(I, 1) . G T .(0.0))$ WRITE(PRINT, 842) I, RLINC(I, 1),

$\& \operatorname{RLINC}(I, 5),(\operatorname{RLINC}(I, K), K=7,9),(\operatorname{RLINC}(I, K), K=11,18)$,

$\&(\operatorname{RLINC}(I, K), K=20,21), \operatorname{NDIV}(I)$

842 FORMAT (1X, I2 , 2X, 'SR' , 3X, F9.1, F6.1,F6.0,F6.1, 7F7.2, 2F8.2,

$\& 2 F 10.2, I 7)$

$\operatorname{IF}(\operatorname{RLINC}(I, 1) . \operatorname{LT} .(0.0))$ WRITE (PRINT, 843) I, TRINC $(I, 1)$

843 FORMAT (1X, I2, 2X, 'SR' , 1X, I2, F9.1)

$\operatorname{IF}(\operatorname{GLINC}(I, 1)$.NE. (0.0)) WRITE(PRINT , 821) I, $\operatorname{GLINC}(I, 1), \operatorname{GLINC}(I, 5)$,

$\&(\operatorname{GLINC}(I, K), K=7,9),(\operatorname{GLINC}(I, K), K=11,18),(\operatorname{GLINC}(I, K), K=20,21)$

821 FORMAT ( $1 \mathrm{X}, \mathrm{I} 2,2 \mathrm{X}$, ' $^{\mathrm{G}} \mathrm{G}$ ' , 3X, F9.1, F6.1, F6.0, F6.1, 7F7.2, 2F8 .2 , 2F10.2)

$\operatorname{IF}((\operatorname{RLINC}(I, 1) \cdot \mathrm{NE} .0$.$) .OR. ( \operatorname{TRINC}(I, 1) \cdot \mathrm{NE} .0.) \cdot \mathrm{OR}$ ( $(\operatorname{GLINC}(I, 1) \cdot \mathrm{NE} .0$.

\&.OR. (ATRIBC $(I, 1) . N E .0)$.$) WRITE (PRINT, 845)$

845 FORMAT $(1 \mathrm{X})$

850 CONTINUE

C $* * * * *$ PRINT TABLE 4B.--SUMMARY OF INPUT LOADS.

C ****** HEADINC

IF(NSEG.GT. 20) WRITE (PRINT, 120) TITLE1, DATE, TITLE2

WRITE (PRINT , 885)

885 FORMAT (/30X,' TABLE 4B. - - SUMMARY OF INPUTS ',

$\&$ 'TO SUBREACHES: LOADS (LB/DAY).'/30X,58(' $\left.{ }^{\prime}\right)$ )

WRITE (PRINT, 890) ((CSHORT $(L, N), N=1,2), L=1,2)$,

$\&((\operatorname{CSHORT}(\mathrm{L}, \mathrm{N}), \mathrm{N}=1,2), \mathrm{L}=4,5)$

890 FORMAT (/1X, ' SUBREACH' , 5X, 'DO' , 3X, 'DO DEF', 3X, 'CBOD' , 3X, 'ORG-N' ,

$\& 2 \mathrm{X},{ }^{\prime} \mathrm{NH} 4-\mathrm{N}^{\prime}, 2 \mathrm{X},{ }^{\prime} \mathrm{NO} 2-\mathrm{N}^{\prime}, 2 \mathrm{X},{ }^{\prime} \mathrm{NO}-\mathrm{N}^{\prime}, 2 \mathrm{X},{ }^{\prime} \mathrm{TOT}-\mathrm{N}^{\prime}, 2 \mathrm{X},{ }^{\prime} \mathrm{O}-\mathrm{P}-\mathrm{A}^{\prime}, 1 \mathrm{X}$,

$\left.\& 4(2 \mathrm{X}, 2 \mathrm{~A} 4), / 1 \mathrm{X}, 132\left({ }^{\prime}-^{\prime}\right) /\right)$

WRITE (PRINT, 895) BEGL(5), (BEGL(K), K=8, 9), (BEGL(K), $K=11,18)$,

$\&(B E G L(K), K=20,21)$

895 FORMAT (/2X,'UPSTREAM:' , 9(F7.0), 4(2X, F8.0),/)

DO $910 \quad I=1$, NSEG

$\operatorname{IF}(\operatorname{ATRIBC}(I, 1)$.NE $(0.0))$ WRITE (PRINT, 900)

$\& I, \operatorname{ATRIBL}(I, 5),(\operatorname{ATRIBL}(I, K), K=8,9),(\operatorname{ATRIBL}(I, K), K=11,18)$,

$\&(\operatorname{ATRIBL}(I, K), K=20,21)$

900 FORMAT(1X, I2,' M TRIB:' , 9F7.0,4(2X, F8.0))

IF (TRINC ( I , 1).GT. (0.0)) WRITE (PRINT, 901) I, TRINL $(I, 5)$,

$\&(\operatorname{TRINL}(I, K), K=8,9),(\operatorname{TRINL}(I, K), K=11,18),(\operatorname{TRINL}(I, K), K=20,21)$

901 FORMAT(1X,I2,' PS/DIV:', 9F7.0,4(2X,F8.0))

IF(RLINC(I,1).GT.0.) WRITE(PRINT , 902)

$\& I, \operatorname{RLINL}(I, 5),(\operatorname{RLINL}(I, K), K=8,9),(\operatorname{RLINL}(I, K), K=11,18)$,

$\&(\operatorname{RLINL}(I, K), \mathrm{K}=20,21)$

902 FORMAT (1X,I2,' S RET: ', 9F7.0,4(2X,F8.0))

MAIN. .

MAIN. .

MAIN. .

MAIN. .

MAIN. .

MAIN. .

MAIN. .

MAIN. .

MAIN. .

MAIN. .

MAIN. .

MAIN. .

MAIN. .

MAIN. .

MAIN. .

MAIN. .

MAIN . .

MAIN. .

MAIN. .

MAIN. .

MAIN. .

MAIN. .

MAIN. .

MAIN. .

MAIN. .

MAIN. .

MAIN. .

MAIN. .

MAIN. .

MAIN. .

MAIN. .

MAIN.

MAIN. .

MAIN. .

MAIN. .

MAIN. .

MAIN.

MAIN. .

MAIN. .

MAIN.

MAIN. .

MAIN. .

MAIN. .

MAIN. .

MAIN. .

MAIN. .

MAIN. .

MAIN. .

MAIN. . 
APPENDIX I--LISTING OF COMPUTER CODE--Continued.

$\operatorname{IF}(\operatorname{GLINC}(I, 1)$.NE. (0.0)) WRITE(PRINT, 903)

$\& I, \operatorname{GLINL}(I, 5),(\operatorname{GLINL}(I, K), K=8,9),(\operatorname{GLINL}(I, K), K=11,18)$,

$\&(\operatorname{GLINL}(I, K), \mathrm{K}=20,21)$

903 FORMAT(1X, I2,' GW RET:', 9F7.0,4(2X, F8.0))

$\operatorname{IF}((\operatorname{RLINC}(I, 1), G T .(0.0))$.OR. (TRINC $(I, 1) . G T .0)$.$.0 R .$

$\&(\operatorname{ATRIBC}(I, 1), G T \cdot 0)$, OR. (GLINC $(I, 1), G T .0)$.$) WRITE(PRINT, 845)$

910 CONTINUE

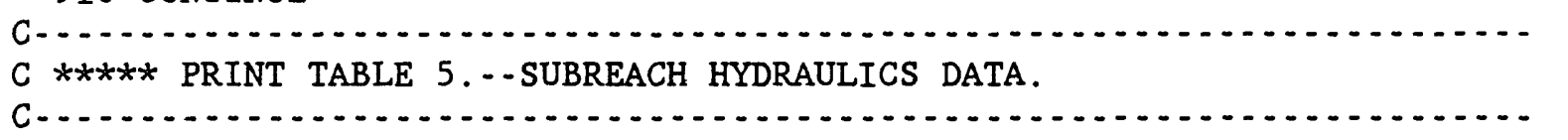

750 CONTINUE

IF((ITAB1.EQ.5) . OR. (ITAB1.EQ.0)) WRITE (PRINT, 120) TITLE1, DATE,

\section{\&TITLE2}

IF ((ITAB1.EQ.5).OR. (ITAB1.EQ.0))WRITE (PRINT, 760)

760 FORMAT (/45X,'TABLE 5...-SUBREACH HYDRAULICS DATA',

$\& / 45 \mathrm{X}, 35\left(^{\prime}-\right.$ - $\left.^{\prime}\right) / / 9 \mathrm{X}$, ' SUBREACH' $, 15 \mathrm{X},{ }^{\prime}$ 'STARTING' , $2 \mathrm{X}$, 'LENGTH' ,

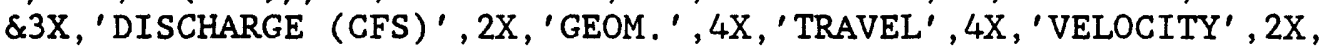

\&'AREA' , 2X, 'DEPTH' , 2X, 'WIDTH' , 2X, 'MEAN SLOPE' ,

$\& / 33 \mathrm{X},,^{\prime} \mathrm{RIVER} \mathrm{R}^{\prime}, 5 \mathrm{X},,^{\prime}(\mathrm{MI})^{\prime}, 4 \mathrm{X}, 14(1 \mathrm{H}-)$,

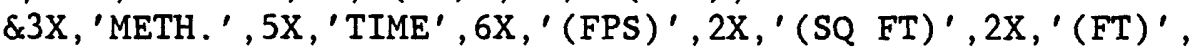

$\& 2 \mathrm{X},,^{\prime}(\mathrm{FT})^{\prime}, 3 \mathrm{X}, 15(1 \mathrm{H}-), / 33 \mathrm{X}, \mathrm{C}^{\prime} \mathrm{MILE}$ ', 15X, 'START' , $4 \mathrm{X}$,

$\&^{\prime} \mathrm{MEAN}^{\prime}, 9 \mathrm{X},{ }^{\prime}(\mathrm{HRS})$ ' , ' (DAYS) ', 31X,'(FT/FT)',1X,'(FT/MI)'/)

762 QSTRT-STARTC(1)

SUM $=0$.

SUMDA $=0$.

DO $790 \mathrm{~J}=1$, NSEG

$\operatorname{FGEOM}(\mathrm{J})=\mathrm{B} 2$

QSTRT-QSTRT+TRINC $(\mathrm{J}, 1)+\mathrm{ATRIBC}(\mathrm{J}, 1)$

IF(QSTRT.LT. (0.0)) WRITE(PRINT, 765)

765 FORMAT (//1X,'TOTAL DISCHARGE WENT NEGATIVE, PROCESSING STOPPED!') IF(QSTRT.LT. (0.0)) GOTO 1520

$\mathrm{QAVG}=\mathrm{QSTRT}+\operatorname{RLINC}(\mathrm{J}, 1) / 2 .+\operatorname{GLINC}(\mathrm{J}, 1) / 2$.

$\operatorname{CALI} \operatorname{CGEOM}(N S E G, \operatorname{GEOM}(\mathrm{J}, 1), \operatorname{GEOM}(\mathrm{J}, 2), \operatorname{GEOM}(\mathrm{J}, 3), \operatorname{GEOM}(\mathrm{J}, 4), \operatorname{GEOM}(\mathrm{J}, 5)$,

$\& \mathrm{VA}(\mathrm{J}), \mathrm{VB}(\mathrm{J}), \mathrm{TA}(\mathrm{J}), \mathrm{TB}(\mathrm{J}), \mathrm{AA}(\mathrm{J}), \mathrm{AB}(\mathrm{J}), \mathrm{DA}(\mathrm{J}), \mathrm{DB}(\mathrm{J}), \mathrm{WA}(\mathrm{J}), \mathrm{WB}(\mathrm{J})$,

\&IGEOM (J ) , QAVG , XLEN (J))

$\operatorname{DAYS}=\operatorname{GEOM}(\mathrm{J}, 2) / 24$.

IF ( (ITAB1.EQ.5) . OR. (ITAB1 .EQ.0))

\&WRITE $(6,780) \mathrm{J},(\operatorname{SNAME}(\mathrm{J}, \mathrm{K}), \mathrm{K}=1,6), \operatorname{BSEG}(\mathrm{J}), \mathrm{XLEN}(\mathrm{J}), \mathrm{QSTRT}, \mathrm{QAVG}$, $\& \operatorname{IGEOM}(\mathrm{J})$, FGEOM $(\mathrm{J})$,

$1 \operatorname{GEOM}(\mathrm{J}, 2), \operatorname{DAYS}, \operatorname{GEOM}(\mathrm{J}, 1),(\operatorname{GEOM}(\mathrm{J}, \mathrm{K}), \mathrm{K}=3,5), \operatorname{SLOPE}(\mathrm{J}), \operatorname{SFPM}(\mathrm{J})$

780 FORMAT (1X, I2 , 2X, 6A4 , 2X, F8. 2, 1X, F8 . 2, 1X, F8.1, 1X, F8.1, 2X, I2, 1A1, 2X,

\&F6.2, 2X, F5.2,

$\& 2 \mathrm{X}, \mathrm{F} 6.2,3 \mathrm{X}, \mathrm{F} 5.0,2 \mathrm{X}, \mathrm{F} 5.1,2 \mathrm{X}, \mathrm{F} 4.0,3 \mathrm{X}, \mathrm{F} 7.5,2 \mathrm{X}, \mathrm{F} 5.2)$

$\operatorname{SUM}=\operatorname{SUM}+\mathrm{GEOM}(\mathrm{J}, 2)$

790 CONTINUE

IF (ILIN.NE .0)QSTRT=QSTRT+RLINC $(\mathrm{J}, 1)+\operatorname{GLINC}(\mathrm{J}, 1)$

SUMDA $=$ SUM $/ 24$

IF ( (ITABI.ES

Q.5).OR. (ITAB1.EQ.0))WRITE (PRINT, 800) QSTRT, SUM, SUMDA MAIN. .

800 FORMAT ( $51 \mathrm{X}, 8\left(^{\prime}-^{\prime}\right), 14 \mathrm{X}, 6\left(^{\prime}-^{\prime}\right), 2 \mathrm{X}, 5\left(^{\prime}-^{\prime}\right), / 49 \mathrm{X}, \mathrm{F} 8.1,^{\prime}, \mathrm{AT}$ END' , 8X, F6 $\& .2,2 \mathrm{X}, \mathrm{F} 5.2, / / 3 \mathrm{X}, '$ (COMPUTATIONAL METHODS - - AREA, DEPTH, WIDTH, VELO

MAIN.

MAIN. . 
APPENDIX I--LISTING OF COMPUTER CODE--Continued.

1CITY AND TRAVELTIME COMPUTED FROM DISCHARGE AND:' $/, 6 \mathrm{X}, 11-\mathrm{VELOCIT}$ 2Y AND DEPTH; 2-- TRAVELTIME AND DEPTH; 3-- AREA AND DEPTH; 4--VEL 3CITY AND WIDTH; 5--TRAVELTIME AND WIDTH; $/, 6 \mathrm{X}, 16$ - - AREA AND WIDTH 4; 7--WIDTH AND DEPTH.' /,6X,'NOTE-- * INDICATES HYDRAULICS DATA WIL \&L BE RECOMPUTED FOR EACH CALCULATION INTERVAL WITHIN THE SUBREACH. $\left.\&)^{\prime}\right)$

$\mathrm{C}$

C $* * * * *$ COMPUTE K2 ONLY FOR SUBREACHES WITH IK2 GT 0

DO $810 \mathrm{~J}=1$, NSEG

IF (IK2(J).EQ.0) GO TO 810

CALL REAER (IK2 (J), $\operatorname{GEOM}(J, 4), \operatorname{GEOM}(J, 1), \operatorname{SLOPE}(J), \operatorname{K2}(\mathrm{J}), \operatorname{CTSVIG}(\mathrm{J})$ )

IF(CTSVIG(J).NE.0) THEN K2FLAG $=1$

810 CONTINUE

IF ( (ITAB1.GT.0) .AND. (ITAB1.NE.6)) GOTO 955

MAIN. .

MAIN. .

MAIN. .

MAIN. .

MAIN. .

MAIN. .

MAIN. .

MAIN. .

MAIN. .

MAIN. .

MAIN. .

MAIN. .

MAIN. .

MAIN. .

MAIN. .

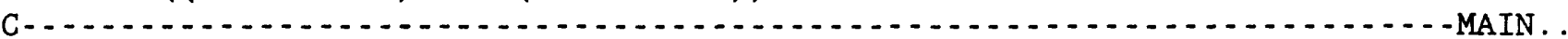

C ***** PRINT TABLE 6.--SUBREACH REACTION COEFFICENTS.

MAIN. .

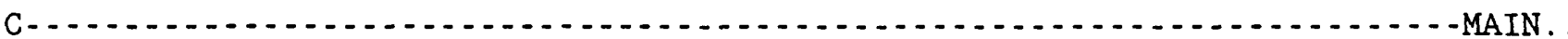

WRITE (PRINT, 120) TITLE1, DATE, TITLE2

WRITE (PRINT, 920)

920 FORMAT (/30X,' TABLE 6.--SUBREACH REACTION COEFFICIENTS.' ,

$\& / 30 \mathrm{X}, 40\left({ }^{\prime}-{ }^{\prime}\right), / 30 \mathrm{X}$, 'REACTION COEFFICIENTS (/DAY AT 20 DEG $\left.\mathrm{C}\right) ' /$ )

WRITE (PRINT, 930) ((CSHORT (L, N), N=1,2), L =4, 5)

930 FORMAT ( $1 \mathrm{X}$, 'S', $2 \mathrm{X}$, 'REAERATION' , 2X, 2('CBOD' , 3X),

$\& 2\left({ }^{\prime} \mathrm{ORG}-\mathrm{N}^{\prime}, 2 \mathrm{X}\right), 2\left({ }^{\prime} \mathrm{NH} 4-\mathrm{N}^{\prime}, 2 \mathrm{X}\right), 2\left({ }^{\prime} \mathrm{NO} 2-\mathrm{N}^{\prime}, 2 \mathrm{X}\right),{ }^{\prime} \mathrm{NO} 3-\mathrm{N}^{\prime}, 1 \mathrm{X}$, , ORTH P-A' ,

$\& 2(2 \mathrm{X}, 2 \mathrm{~A} 4) / 1 \mathrm{X},,^{\prime} \mathrm{U}^{\prime}, 2 \mathrm{X}, \mathrm{\prime}^{\prime} \mathrm{ME}-{ }^{\prime}, 2 \mathrm{X},{ }^{\prime} \mathrm{K} 2{ }^{\prime}, 2 \mathrm{X}, 4$ (3X, 'DECAY' ,

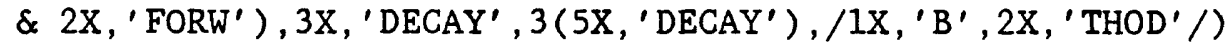

WRITE (PRINT, 940) ( J , IK2 (J), K2 (J), KCR (J), KC (J),

$\& \operatorname{KORNR}(\mathrm{J}), \operatorname{KORNF}(\mathrm{J}), \operatorname{KNH} 4 \mathrm{R}(\mathrm{J}), \operatorname{KNH} 4 \mathrm{~F}(\mathrm{~J}), \operatorname{KNO} 2 R(\mathrm{~J})$,

$\& \operatorname{KNO} 2 F(J), \operatorname{KNO} 3 R(J), \operatorname{KPO} 4 B(J), \operatorname{KNCR} 1(\mathrm{~J}), \operatorname{KNCR} 2(\mathrm{~J}), \mathrm{J}=1, \mathrm{NSEG})$

940 FORMAT (1X, I2 , 1X, I2 , 1X, F6 . 2, 9F7.2, 3F10.2)

WRITE (PRINT, 950)

950 FORMAT (/,10X,'METHODS FOR CALCULATING K2: 0--INPUT AS DATA; $1--\mathrm{B}$

\&ENNETT/RATHBUN; 2--VELZ| 3--LANGBEIN/DURHAM| 4--PADDEN-GLOYNA |', /1

\&5X,' 5--BANSAL; 6--PARKHURST-POMEROY| 7 --TSIVOGLOU -WALLACE| 12--MOD

\&IFIED TSIVOGLOU-WALLACE' )

IF (K2FLAG.NE.0) WRITE (PRINT, 952)

952 FORMAT (' (NON-STANDARD ESCAPE COEFFICIENTS)')

WRITE (PRINT, 954)

954 FORMAT $\left({ }^{\prime} . '\right)$

C $* * * * *$ MAKE TEMPERATURE CORRECTIONS TO REACTION COEFFICIENTS (JON 6/81)

955 DO $960 \mathrm{~J}=1$, NSEG

$\operatorname{DTEMP}=\operatorname{TEMP}(\mathrm{J})-20$.

C $* * * * *$ THETA $=1.0241$ [ELMORE AND WEST, 1961]

$\mathrm{XX}=1.0241 * * \mathrm{DTEMP}$

$\mathrm{K} 2(\mathrm{~J})=\mathrm{K} 2(\mathrm{~J}) * \mathrm{XX}$

C ***** THETA- 1.047 [SHINDALA, 1972]

$\mathrm{XX}-1.047 * *$ DTEMP

$\mathrm{KC}(\mathrm{J})=\mathrm{KC}(\mathrm{J}) * \mathrm{XX}$

$\mathrm{KCR}(\mathrm{J})=\mathrm{KCR}(\mathrm{J}) * \mathrm{XX}$

MAIN. .

MAIN. .

MAIN. .

MAIN. .

MAIN. .

MAIN. .

MAIN. .

MAIN. .

MAIN. .

MAIN. .

MAIN. .

MAIN. .

MAIN. .

MAIN. .

MAIN. .

MAIN. .

MAIN. .

MAIN. .

MAIN. .

MAIN. .

MAIN. .

MAIN. .

MAIN. .

MAIN. .

MAIN. .

MAIN. .

MAIN. .

MAIN. .

MAIN. .

MAIN. .

MAIN. . 
APPENDIX I--LISTING OF COMPUTER CODE--Continued.

C $* * * * *$ THETA $=1.09$

MAIN. .

$\mathrm{XX}=1.09 * * \mathrm{DTEMP}$

$\operatorname{KNCR} 1(\mathrm{~J})=\operatorname{KNCR} 1(\mathrm{~J}) * \mathrm{XX}$

$\operatorname{KNCR} 2(\mathrm{~J})=\mathrm{KNCR} 2(\mathrm{~J}) * \mathrm{XX}$

$\mathrm{KPO} 4 \mathrm{~B}(\mathrm{~J})=\mathrm{KPO} 4 \mathrm{~B}(\mathrm{~J}) * \mathrm{XX}$

$\operatorname{KORNF}(J)=\operatorname{KORNF}(J) * X X$

$\mathrm{KNH} 4 \mathrm{~F}(\mathrm{~J})=\mathrm{KNH} 4 \mathrm{~F}(\mathrm{~J}) * \mathrm{XX}$

$\mathrm{KNO} 2 \mathrm{~F}(\mathrm{~J})=\mathrm{KNO} 2 \mathrm{~F}(\mathrm{~J}) * \mathrm{XX}$

$\operatorname{KNO3R}(\mathrm{J})=\mathrm{KNO} 3 \mathrm{R}(\mathrm{J}) * \mathrm{XX}$

$\operatorname{KORNR}(\mathrm{J})=\operatorname{KORNR}(\mathrm{J}) * \mathrm{XX}$

$\mathrm{KNH} 4 \mathrm{R}(\mathrm{J})=\mathrm{KNH} 4 \mathrm{R}(\mathrm{J}) * \mathrm{XX}$

$\mathrm{KNO} 2 \mathrm{R}(\mathrm{J})=\mathrm{KNO} 2 \mathrm{R}(\mathrm{J}) * \mathrm{XX}$

$\mathrm{C} * * * * *$ THETA $=1.065$

$\mathrm{XX}=1.065 * *$ DTEMP

$\mathrm{BN}(\mathrm{J})=\mathrm{BN}(\mathrm{J}) * \mathrm{XX}$

960 CONTINUE

MAIN. .

MAIN. .

MAIN. .

MAIN. .

MAIN. .

MAIN. .

MAIN. .

MAIN. .

MAIN. .

MAIN. .

MAIN. .

MAIN. .

MAIN. .

MAIN. .

MAIN. .

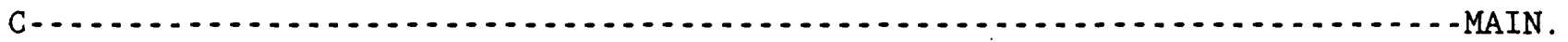

C $* * * * *$ PRINT TABLE OF TEMP CORRECTED REACTION COEFFICIENTS

MAIN. .

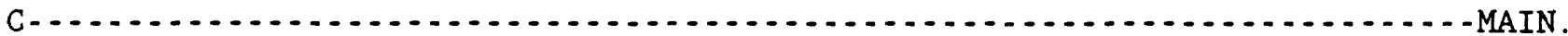

IF ( (ITAB1.NE .0) . AND. (ITAB1.NE.6)) GOTO 1005

IF(JSEG.EQ.0) WRITE (PRINT, 120) TITLE1, DATE, TITLE2

WRITE (PRINT, 970)

970 FORMAT (/30X, 'TABLE 6.--SUBREACH REACTION COEFFICIENTS',

$\&{ }^{\prime}$--CONTINUED. $\left.{ }^{\prime}, / 30 \mathrm{X}, 52\left({ }^{\prime}-{ }^{\prime}\right)\right)$

WRITE (PRINT, 980)

980 FORMAT (30X, 'TEMPERATURE-CORRECTED REACTION COEFFICIENTS (/DAY',

$\&$ ' AT AMBIENT TEMPERATURE)' $/$ )

WRITE (PRINT, 990) ((CSHORT (L,N), N=1, 2), L-4, 5)

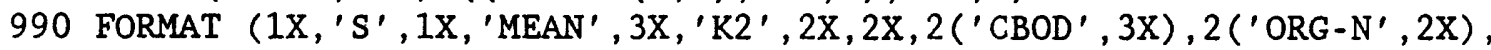

$\& 2\left({ }^{\prime} \mathrm{NH} 4-\mathrm{N}^{\prime}, 2 \mathrm{X}\right), 2\left({ }^{\prime} \mathrm{NO} 2-\mathrm{N}^{\prime}, 2 \mathrm{X}\right),{ }^{\prime} \mathrm{NO}-\mathrm{N}^{\prime}, 1 \mathrm{X},{ }^{\prime} \mathrm{ORTHO}-\mathrm{P}-\mathrm{A}^{\prime}, 2(2 \mathrm{X}, 2 \mathrm{~A} 4)$,

$\& /, 1 \mathrm{X},{ }^{\prime} \mathrm{U}^{\prime}, 1 \mathrm{X},{ }^{\prime} \mathrm{TEMP} . ', 5 \mathrm{X}, 4$ (3X, 'DECAY' , 2X, 'FORW' ), 3X, 'DECAY' ,

$\left.\& 3\left(5 \mathrm{X},{ }^{\prime} \mathrm{DECAY}^{\prime}\right), /, 1 \mathrm{X}, \mathrm{B}^{\prime}, 2 \mathrm{X},{ }^{\prime}(\mathrm{C})^{\prime}, /\right)$

WRITE (PRINT , 1000) (J, TEMP(J), K2(J), KCR(J), KC(J),

$\& \operatorname{KORNR}(J), \operatorname{KORNF}(J), \operatorname{KNH} 4 R(J), \operatorname{KNH} 4 F(J), \operatorname{KNO} 2 R(J)$,

$\& \operatorname{KNO} 2 \mathrm{~F}(\mathrm{~J}), \operatorname{KNO} 3 R(\mathrm{~J}), \operatorname{KPO} 4 \mathrm{~B}(\mathrm{~J}), \operatorname{KNCR} 1(\mathrm{~J}), \mathrm{KNCR} 2(\mathrm{~J}), \mathrm{J}=1, \mathrm{NSEG})$

1000 FORMAT (1X, I2, 1X, F4.1, 10F7.2, 3F10.2)

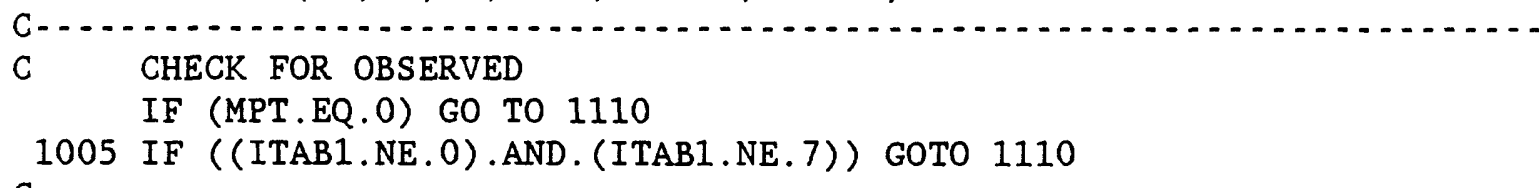

C $* * * * *$ PRINT TABLE 7. -OBSERVED WATER-QUALITY DATA.

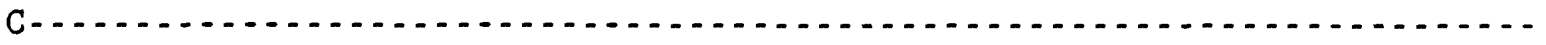

LNCNTR $=60$

NOLD $=0$

OLDMI $=0$.

$\mathrm{NS}=1$

$\mathrm{C} * * * * *$

DO $1100 \mathrm{I}=1, \mathrm{MPT}$

C CALCULATE DIS N/P RATIO (MOLES/MOLE

MAIN.

MAIN. .

MAIN. .

MAIN. .

MAIN. .

MAIN. .

MAIN. .

MAIN . .

MAIN. .

MAIN. .

MAIN. .

MAIN. .

MAIN. .

MAIN. .

MAIN. .

MAIN. .

MAIN. .

MAIN. .

MAIN. .

MAIN.

MAIN. .

MAIN. .

MAIN. .

MAIN. .

MAIN. .

MAIN. .

MAIN. .

MAIN. .

MAIN. .

MAIN. .

MAIN. . 
APPENDIX I--LISTING OF COMPUTER CODE--Continued.

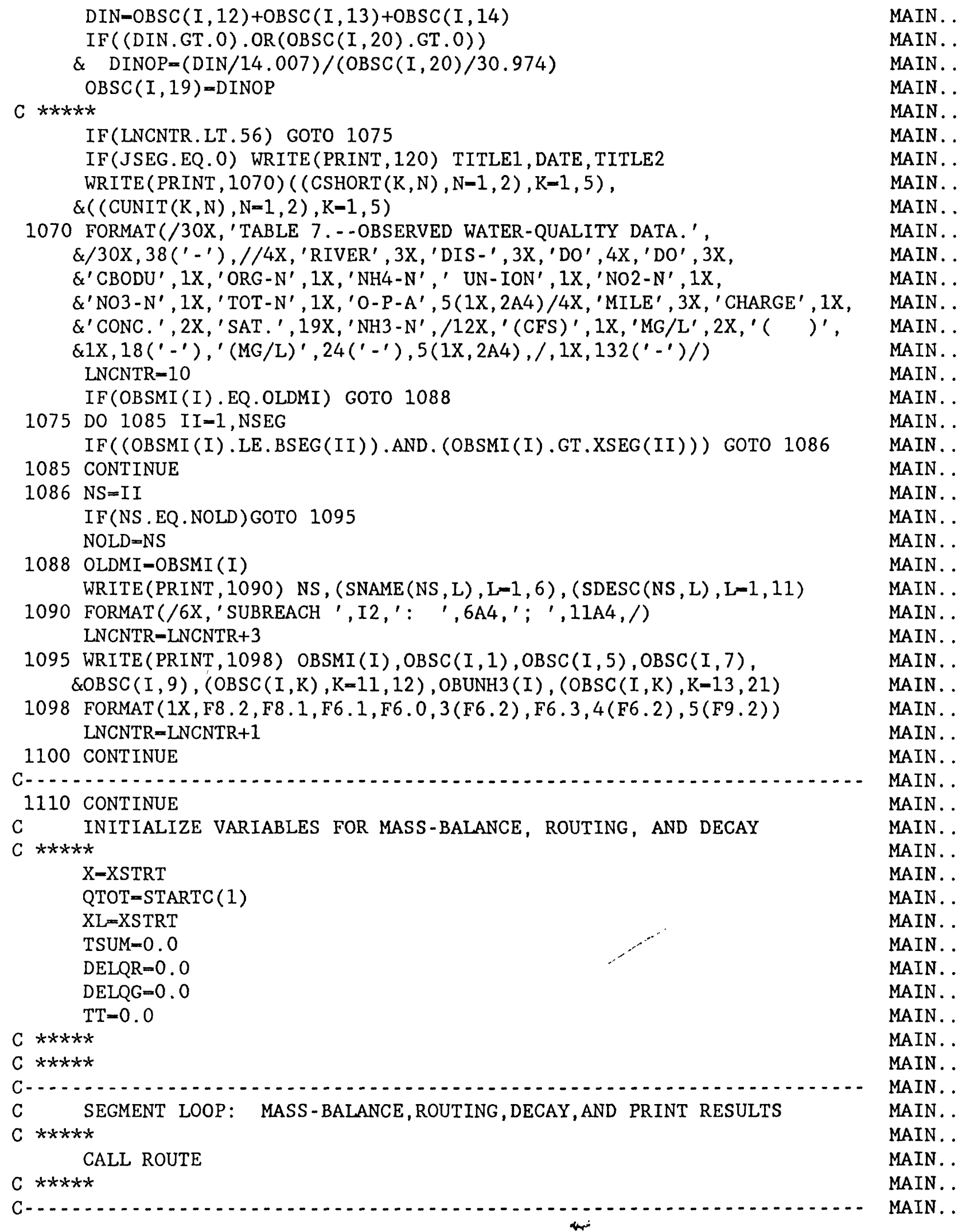


APPENDIX I--LISTING OF COMPUTER CODE--Continued.

C $* * * *$ PRINT AND/OR PLOT RESULTS OF COMPUTATIONS:

MAIN. .

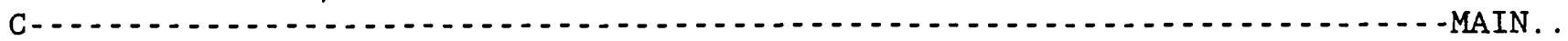

C $* * * * *$ PRINT TABLE 4A.--SUMMARY OF INPUTS TO SUBREACHES: CONCENTRATIONS. MAIN..

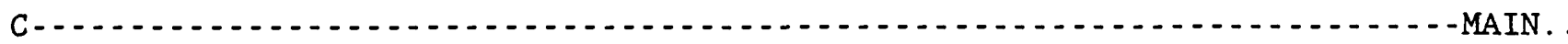

C ****** HEADING

IF ( (ITAB1.NE.0). AND. (ITAB1.NE.4)) GOTO 8910

IF (ITFLAG.EQ.0) GOTO 8910

WRITE (PRINT, 120) TITLE1, DATE, TITLE2

WRITE (PRINT, 8820)

8820 FORMAT (/30X, 'REVISED TABLE 4A.--SUMMARY OF INPUTS',

$\&$ ' TO SUBREACHES: CONCENTRATIONS.'/30X,60(' $\left.-^{\prime}\right)$ )

WRITE (PRINT, 830) ((CSHORT $(\mathrm{L}, \mathrm{N}), \mathrm{N}=1,2), \mathrm{L}=1,2)$,

C ****** CONCENTRATIONS

$\&((\operatorname{CSHORT}(\mathrm{L}, \mathrm{N}), \mathrm{N}=1,2), \mathrm{L}=4,5)$

WRITE (PRINT, 835) $\operatorname{BEGC}(1), \operatorname{BEGC}(5),(\operatorname{BEGC}(\mathrm{K}), \mathrm{K}=7,9)$,

$\&(\operatorname{BEGC}(K), K=11,18),(\operatorname{BEGC}(K), K=20,21)$

DO $8850 \mathrm{I}=1$, NSEG

$\operatorname{IF}(\operatorname{ATRIBC}(I, 1), G T .(0.0))$ WRITE(PRINT, 839) I, ATRIBC $(I, 1)$,

$\& \operatorname{ATRIBC}(I, 5),(\operatorname{ATRIBC}(I, K), K=7,9),(\operatorname{ATRIBC}(I, K), K=11,18)$,

$\&$ (ATRIBC $(I, K), K=20,21$ )

$\operatorname{IF}(\operatorname{TRINC}(I, 1) . G T .(0.0))$ WRITE (PRINT, 840) I, $\operatorname{TRINC}(I, 1), \operatorname{TRINC}(I, 5)$,

$\&(\operatorname{TRINC}(I, K), K=7,9),(\operatorname{TRINC}(I, K), K=11,18),(\operatorname{TRINC}(I, K), K=20,21)$

$\operatorname{IF}(\operatorname{TRINC}(I, 1)$. LT . (0.0)) WRITE (PRINT , 841) I, TRINC ( I, 1)

IF(ILIN.EQ. (0)) GOTO 8850

IF(RLINC (I, 1).GT. (0.0)) WRITE(PRINT, 842) I, RLINC(I, 1),

$\& \operatorname{RLINC}(I, 5),(\operatorname{RLINC}(I, K), K=7,9),(\operatorname{RLINC}(I, K), K=11,18)$,

$\&(\operatorname{RLINC}(I, K), K=20,21), \operatorname{NDIV}(I)$

$\operatorname{IF}(\operatorname{RLINC}(I, 1) . \operatorname{LT} .(0.0))$ WRITE (PRINT, 843) I, TRINC $(I, 1)$

$\operatorname{IF}(\operatorname{GLINC}(I, 1) . N E .(0.0))$ WRITE(PRINT, 821) I, GLINC $(I, 1), \operatorname{GLINC}(I, 5)$,

\& (GLINC $(I, K), K=7,9),(\operatorname{GLINC}(I, K), K=11,18),(\operatorname{GLINC}(I, K), K=20,21)$

MAIN. .

MAIN. .

MAIN. .

MAIN. .

MAIN. .

MAIN. .

MAIN. .

MAIN. .

MAIN. .

MAIN. .

MAIN. .

MAIN. .

MAIN. .

MAIN. .

MAIN. .

MAIN. .

MAIN. .

MAIN. .

MAIN. .

MAIN. .

MAIN. .

MAIN. .

MAIN. .

MAIN. .

MAIN. .

$\operatorname{IF}((\operatorname{RLINC}(I, 1) \cdot N E .0$.$) .OR.(TRINC (I, 1) \cdot N E .0$.$) .OR. (GLINC (I, 1) \cdot N E .0$.

MAIN. .

\&.OR. (ATRIBC $(I, 1)$.NE.0.)) WRITE (PRINT, 845)

8850 CONTINUE

MAIN. .

MAIN. .

MAIN. .

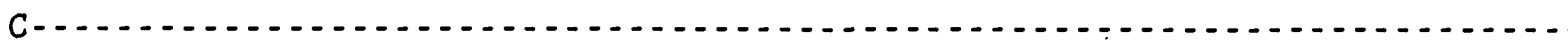

C $* * * * *$ PRINT TABLE 4B.--REVISED SUMMARY OF INPUT LOADS.

MAIN.

$C * * * * *$ HEADING

IF(NSEG.GT. 20) WRITE (PRINT, 120) TITLE1, DATE, TITLE2

WRITE (PRINT , 8885)

8885 FORMAT (/30X, 'REVISED TABLE 4B.--SUMMARY OF INPUTS',

$\&$ ' SUBREACHES : LOADS . '/30X, 50 (' $\left.{ }^{\prime}\right)$ )

WRITE (PRINT, 890) ((CSHORT $(L, N), N=1,2), L=1,2)$,

$\&((\operatorname{CSHORT}(\mathrm{L}, \mathrm{N}), \mathrm{N}=1,2), \mathrm{L}=4,5)$

WRITE (PRINT, 895) BEGL (5), (BEGL(K), $K=8,9$ ), (BEGL (K), $K=11,18$ ),

$\&$ (BEGL $(\mathrm{K}), \mathrm{K}=20,21$ )

DO $8909 \mathrm{I}=1$, NSEG

$\operatorname{IF}(\operatorname{ATRIBC}(I, 1)$.NE. (0.0)) WRITE(PRINT, 900)

$\& I, \operatorname{ATRIBL}(I, 5),(\operatorname{ATRIBL}(I, K), K=8,9),(\operatorname{ATRIBL}(I, K), K=11,18)$,

$\&$ (ATRIBL $(I, K), K=20,21$ )

IF (TRINC (I, 1).GT. (0.0))WRITE (PRINT, 901) I, TRINL (I, 5),

MAIN. .

MAIN .

MAIN.

MAIN. .

MAIN. .

MAIN. .

MAIN. .

MAIN. .

MAIN. .

MAIN. .

MAIN. .

MAIN. .

MAIN. .

MAIN. .

MAIN. . 
APPENDIX I--LISTING OF COMPUTER CODE--Continued.

$\&(\operatorname{TRINL}(I, K), K=8,9),(\operatorname{TRINL}(I, K), K=11,18),(\operatorname{TRINL}(I, K), K=20,21)$

MAIN.

IF (RLINC (I, 1).GT.0.) WRITE(PRINT, 902)

$\& I, \operatorname{RLINL}(I, 5),(\operatorname{RLINL}(I, K), K=8,9),(\operatorname{RLINL}(I, K), K=11,18)$,

$\&(\operatorname{RLINL}(I, K), \mathrm{K}=20,21)$

IF(GLINC(I,1).NE. (0.0)) WRITE(PRINT, 903)

$\& I, \operatorname{GLINL}(I, 5),(\operatorname{GLINL}(I, K), K=8,9),(\operatorname{GLINL}(I, K), K=11,18)$,

$\&(\operatorname{GLINL}(I, K), K=20,21)$

$\operatorname{IF}((\operatorname{RLINC}(I, 1), G T \cdot(0.0))$.OR. (TRINC $(I, 1) . G T .0)$.$.0 R .$

$\&(\operatorname{ATRIBC}(I, 1) . \mathrm{GT} .0.) . \mathrm{OR} .(\operatorname{GLINC}(I, 1) . \mathrm{GT} .0)$.$) WRITE(PRINT , 845)$

8909 CONTINUE

8910 CONTINUE

MAIN. .

MAIN. .

MAIN. .

MAIN. .

MAIN. .

MAIN. .

MAIN. .

MAIN. .

MAIN. .

MAIN. .

IF (IGRAPH.NE.1)GO TO 8917

MAIN. .

MAIN. .

C

C $* * * * *$ OUTPUT A DATA ARRAY FOR POST-PROCESSING BY EXTERNAL PROGRAMS

C TO PRODUCE PUBLICATION-QUALITY PLOTS

C SUBSEQUENT FILE HANDLING BY EXTERNAL PRIMOS CPL PROGRAMS

C

$C * * * * *$ OPEN AND WRITE OUTPUT PLOTFILES

OPEN ( 11, FILE=' PLOTOUT' , STATUS=' UNKNOWN')

C

DO $8916 I=1$, JJ

WRITE $(11,8915) \operatorname{XDIST}(I), \operatorname{PLOTC}(I, 1), \operatorname{PLOTC}(I, 5), \operatorname{PLOTC}(I, 6), \operatorname{PLOTC}(I$,

\&7), $\operatorname{PLOTC}(I, 9), \operatorname{PLOTC}(I, 11), \operatorname{PLOTC}(I, 12), \operatorname{PLOTC}(I, 30), \operatorname{PGEOM}(I, 2), \operatorname{PL}$

\&OTC $(I, 18), \operatorname{PLOTC}(I, 19), \operatorname{PLOTC}(I, 20), \operatorname{PLOTC}(I, 21), \operatorname{PLOTC}(I, 13), \operatorname{PLOTC}($

$\& I, 14), \operatorname{PLOTC}(I, 15), \operatorname{PLOTC}(I, 16), \operatorname{UNH} 3(I)$

8916 CONTINUE

DO 8930 II $=1$, MPT

WRITE $(11,8914)$ OBSMI (II), OBSC (II, 1), OBSC (II , 5), OBSC (II , 6),

\&OBSC (II , 7) , OBSC (II , 9), OBSC (II , 11), OBSC (II , 12), OBSC (II , 18),

\&OBSC (II , 20), OBSC (II , 21), OBSC (II , 13), OBSC (II , 14), OBSC (II , 15),

\&OBSC (II , 16), OBUNH3 (II)

8930 CONTINUE

8914 FORMAT (F6.2, F6.1,13(F6.2), F6.3)

8915 FORMAT (F6.2,F6.0,16(F6.2),F6.3)

MAIN. .

MAIN. .

MAIN. .

MAIN. .

MAIN. .

MAIN. .

MAIN. .

MAIN. .

MAIN. .

MAIN. .

MAIN. .

MAIN. .

MAIN. .

MAIN. .

MAIN. .

MAIN. .

MAIN. .

MAIN. .

MAIN. .

MAIN. .

MAIN. .

MAIN. .

MAIN. .

8917 IF(IPLOT.EQ. 99) GOTO 1490

C $* * * * *$ PERFORM LINE-PRINTER PLOTS

C $* * * * *$ NPLOT SPECIFIES PLOT PARAMETERS:

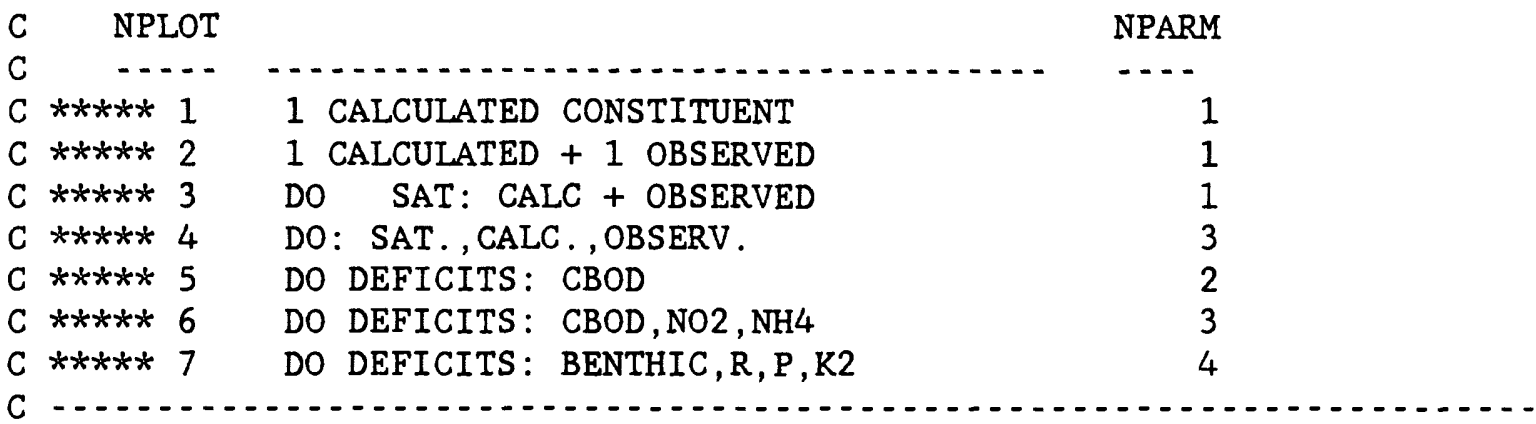

Do $9005 \mathrm{I}=1, \mathrm{JJ}$

MAIN. .

MAIN. .

MAIN. .

MAIN. .

MAIN. .

MAIN. .

MAIN. .

MAIN. .

MAIN. .

MAIN. .

MAIN. .

MAIN. .

MAIN. .

MAIN. . 
APPENDIX I--LISTING OF COMPUTER CODE--Continued.

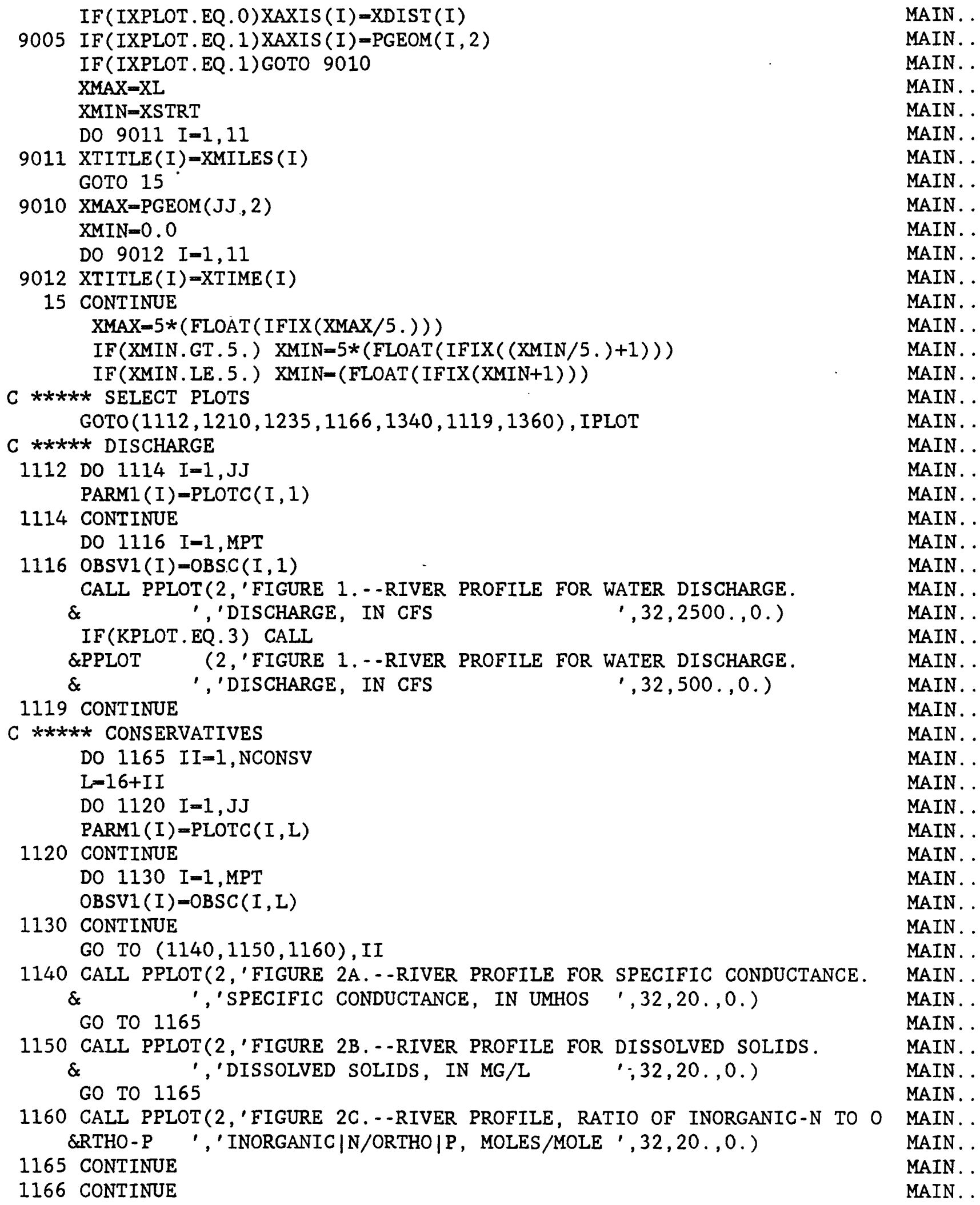

MAIN. .

MAIN. .

MAIN. .

MAIN. .

MAIN. .

MAIN. .

MAIN. .

MAIN. .

MAIN. .

MAIN. .

MAIN. .

MAIN. .

MAIN. .

MAIN. .

MAIN. .

MAIN. .

MAIN. .

MAIN. .

MAIN. .

MAIN. .

MAIN. .

MAIN. .

MAIN. .

MAIN. .

MAIN. .

MAIN. .

MAIN. .

MAIN. .

MAIN. .

MAIN. .

MAIN. .

MAIN. .

MAIN. .

MAIN. .

MAIN. .

MAIN. .

MAIN. .

MAIN. .

MAIN. .

MAIN. .

MAIN. .

MAIN. .

MAIN. .

MAIN. .

MAIN. .

MAIN. .

MAIN. .

MAIN. .

MAIN. .

MAIN. . 
APPENDIX I--LISTING OF COMPUTER CODE--Continued.

IF(IPLOT.EQ.6) GOTO 1490

C $* * * * *$ DO PARAMETERS

DO $1170 \quad I=1, \mathrm{JJ}$

$\operatorname{PARM1}(I)=\operatorname{PLOTC}(I, 6)$

$\operatorname{PARM} 2(I)=\operatorname{PLOTC}(I, 5)$

1170 CONTINUE

DO $1180 \quad I=1, M P T$

$\operatorname{OBSV1}(I)=\operatorname{OBSC}(I, 5)$

1180 CONTINUE

CALL PPLOT (3,'FIGURE 3.--RIVER PROFILE FOR DO CONCENTRATIONS.

$\& \quad '$, DISSOLVED OXYGEN, IN MG/L ',32,20.,0.)

DO $1190 \mathrm{I}=1, \mathrm{JJ}$

$\operatorname{PARMI}(I)=100$.

$\operatorname{PARM} 2(I)=\operatorname{PLOTC}(I, 7)$

1190 CONTINUE

DO $1200 \quad I=1, M P T$

$\operatorname{OBSV} 1(I)=\operatorname{OBSC}(I, 7)$

1200 CONTINUE

CALL PPLOT ( 3, 'FIGURE 4.--RIVER PROFILE FOR DISSOLVED-OXYGEN SATURA

\&TION. ','DO SATURATION, IN PERCENT ', 32, 250.,0.)

C ****** DO DEFICITS

DO $1202 \mathrm{I}=1, \mathrm{JJ}$

$1202 \operatorname{PARM} 1(I)=\operatorname{PLOTC}(I, 8)$

CALL PPLOT( $1, '$ FIGURE 5A.--RIVER PROFILE FOR TOTAL DO DEFICITS.

\&DEFICITS.' ,'DO DEFICITS, IN MG/L

' $32,2.0,0$.

IF (IMODEL.EQ. 1) GOTO 1206

DO $1204 \mathrm{I}=1$, JJ

$\operatorname{PARM} 1$ (I) $=\operatorname{PLOTC}(I, 23)$

$\operatorname{PARM} 2(I)=\operatorname{PLOTC}(I, 24)$

$1204 \operatorname{PARM} 3(I)=\operatorname{PLOTC}(I, 8)$

CALL PPLOT (5,'FIGURE 5B.--RIVER PROFILE FOR DISSOLVED-OXYGEN DEFIC

\&ITS . ','DO DEFICITS, IN MG/L

$,, 32, .1,0$. GOTO 1208

1206 DO $1207 \mathrm{I}=1$, JJ

PARM1 (I) $=$ PLOTC $(I, 23)$

$\operatorname{PARM} 2(I)=\operatorname{PLOTC}(I, 26)$

$\operatorname{PARM} 3(I)=P L O T C(I, 25)$

1207 CONTINUE

CALL PPLOT (6,'FIGURE 5B.--RIVER PROFILE FOR DISSOLVED-OXYGEN DEFIC \&ITS. ', 'DO DEFICITS, IN MG/L

$,, 32, .1,0$.

1208 DO $1209 \mathrm{I}=1, \mathrm{JJ}$

PARMI (I) $=$ PLOTC ( I , 29)

$\operatorname{PARM} 2(I)=\operatorname{PLOTC}(I, 28)$

$\operatorname{PARM} 3(I)=-1 . * \operatorname{PLOTC}(I, 27)$

$\operatorname{PARM} 4(I)=-1 . * \operatorname{PLOTC}(I, 22)$

1209 CONTINUE

CALL PPLOT( $7, '$ FIGURE 5C.--RIVER PROFILE FOR DISSOLVED-OXYGEN DEFIC \&ITS . $'$, DO DEFICITS, IN MG/L $,, 32, .1,0$.

$\mathrm{C} * * * * * *$ CBOD

1210 DO $1220 \mathrm{I}=1, \mathrm{JJ}$

MAIN. .

MAIN. .

MAIN. .

MAIN. .

MAIN. .

MAIN. .

MAIN. .

MAIN. .

MAIN. .

MAIN. .

MAIN. .

MAIN. .

MAIN. .

MAIN. .

MAIN. .

MAIN. .

MAIN. .

MAIN. .

MAIN. .

MAIN. .

MAIN. .

MAIN. .

MAIN. .

MAIN. .

MAIN. .

MAIN. .

MAIN. .

MAIN . .

MAIN. .

MAIN. .

MAIN. .

MAIN. .

MAIN. .

MAIN. .

MAIN. .

MAIN. .

MAIN. .

MAIN. .

MAIN. .

MAIN. .

MAIN. .

MAIN. .

MAIN. .

MAIN . .

MAIN. .

MAIN. .

MAIN. .

MAIN. .

MAIN. .

MAIN. . 
APPENDIX I--LISTING OF COMPUTER CODE--Continued.

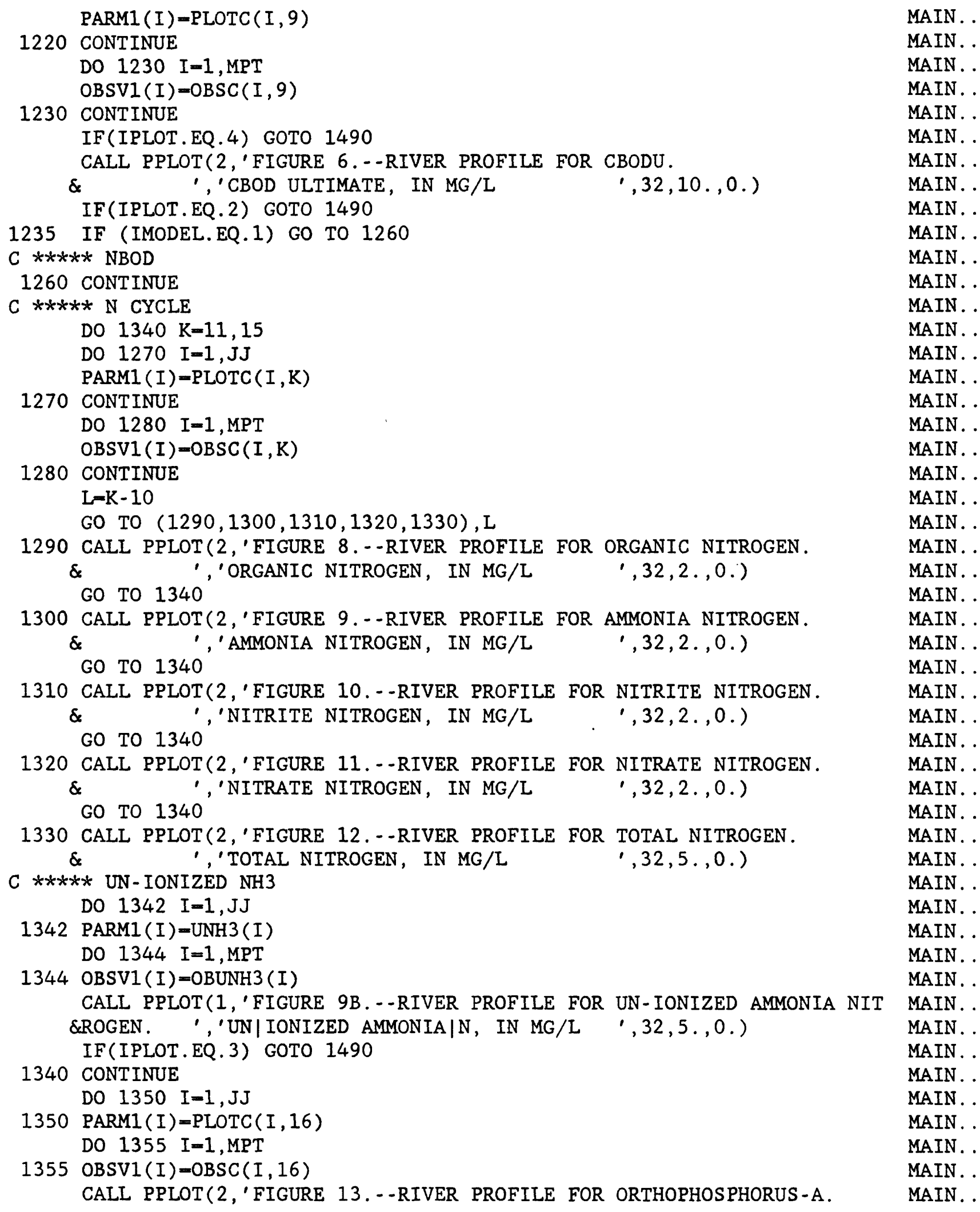

MAIN .

MAIN. .

MAIN. .

MAIN. .

MAIN. .

MAIN. .

MAIN.

MAIN. .

MAIN.

MAIN.

MAIN. .

MAIN.

MAIN. .

MAIN.

MAIN.

MAIN. .

MAIN.

MAIN. .

MAIN. .

MAIN. .

MAIN .

MAIN.

MAIN .

MAIN. .

MAIN.

MAIN. .

MAIN .

MAIN. .

MAIN. .

MAIN .

MAIN.

MAIN. .

MAIN. .

MAIN. .

MAIN. .

MAIN. .

MAIN. .

MAIN. .

MAIN. .

MAIN. .

MAIN. .

MAIN.

MAIN. .

MAIN.

MAIN. .

MAIN. .

MAIN. .

MAIN.

MAIN. .

MAIN. . 
APPENDIX I--LISTING OF COMPUTER CODE--Continued.

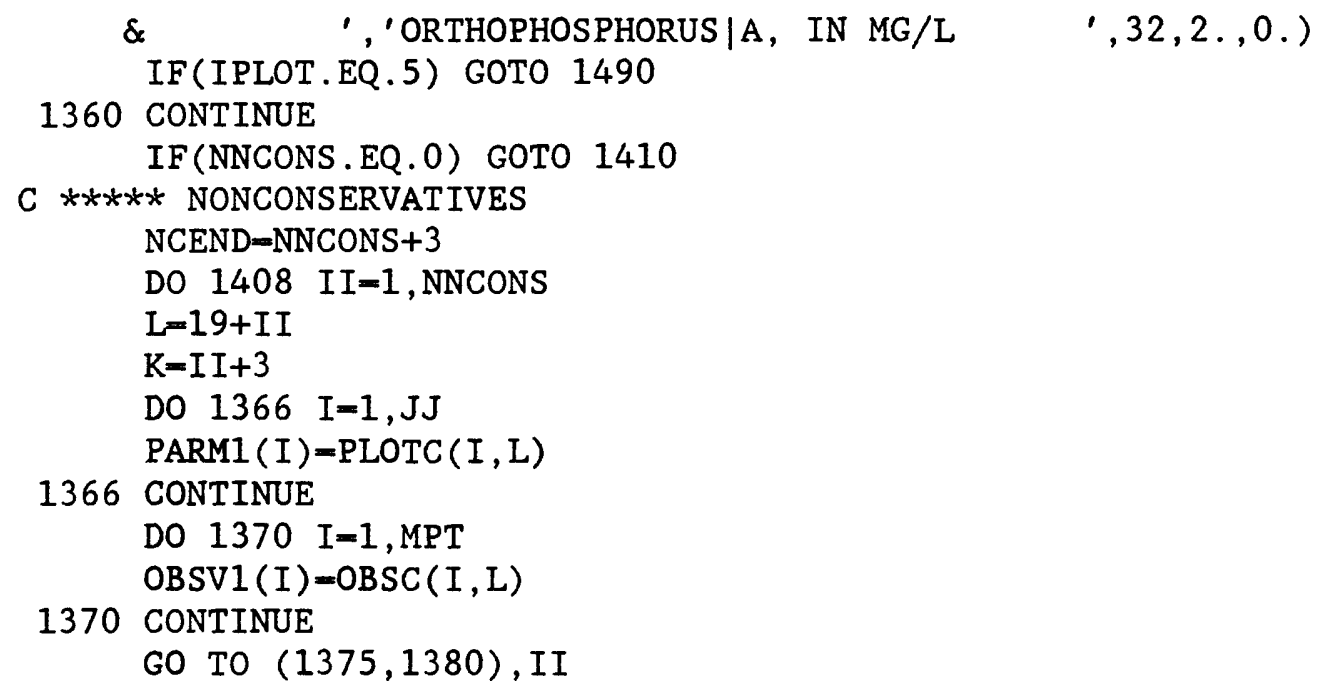

1440 CALL PPLOT(1,'FIGURE 17.--RIVER PROFILE FOR MEAN VELOCITY.

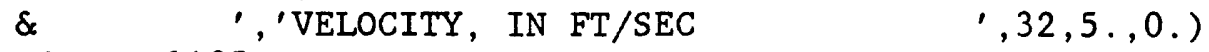

1450 CALL PPLOT(1,'FIGURE 18.--RIVER PROFILE FOR CUMULATIVE TRAVELTIME \&. ','TRAVELTIME, IN DAYS ',32,15.,0.) GO TO 1485

1460 CALL PPLOT(1,'FIGURE 19.--RIVER PROFILE FOR MEAN CROSS-SECTIONAL A \&REA. ','CROSS|SECTIONAL AREA, IN SQ FT ', 32,1000.,0.) GO TO 1485

1470 CALL PPLOT(1,'FIGURE 20.--RIVER PROFILE FOR MEAN CHANNEL DEPTH. $\&$ ', 'DEPTH, IN FEET ',32,10.,0.) GO TO 1485

1480 CALL PPLOT(1,'FIGURE 21. --RIVER PROFILE FOR MEAN CHANNEL WIDTH. $\&$ 1485 CONTINUE ','CHANNEL WIDTH, IN FEET ',32,500.,0.) 1490 CONTINUE 
APPENDIX I--LISTING OF COMPUTER CODE--Continued.

1500 IF (ISWBAD.EQ. 1)WRITE (PRINT, 1510)

1510 FORMAT(1H1,'ERROR HAS OCCURRED IN INPUT DATA, PROCESSING ENDED.')

C ***** TEST FOR END OF MAINSTEM CALCULATIONS
IF((IEND. EQ. 0) . AND. (ISWBAD. NE. 1)) GOTO 50
CLOSE (5)
CLOSE (6)
CLOSE (11)
STOP
END

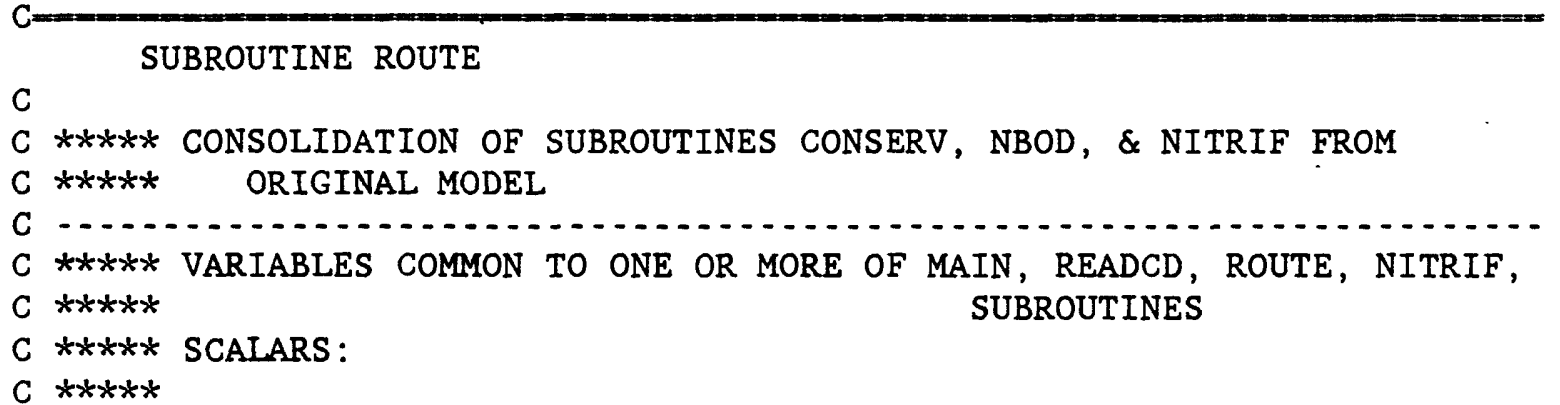

COMMON /VALL/ BDN, BNT, CARD, DELNH4, DELNO2, DELTT, DXPNT, EA,

1 ER, ICDBUF, IEND, IGRAPH, ITYPE, ILIN, IMODEL, INITSW, IPLOT,

2 IPO4, IPRMIN, IPNET, IRET, ISW1, ISWBAD, ITAB1, ITAB2, ITEST,

3 ITFLAG, IXGEOM, J, JJ, JSEG, KPLOT, K2T, KCT, KCRT, KORNRT,

4 KORNFT, KNH4RT, KNH4FT, KNO2RT, KNO2FT, KNO3RT, KPO4BT, KNCR1T,

5 KNCR2T, LNCNTR, MPT, NCONSV, NNCONS, NSEG, PRINT, QTOT, TT,

$C * * * * * *$

6 TTSUM, XEND, XL, XSTRT, IRESP, DATE

C ****** ARRAYS:

$C * * * * * *$

COMMON /ALL/ $\operatorname{AA}(50), \operatorname{AB}(50), \operatorname{ATRIBC}(50,21), \operatorname{ATRIBL}(50,21), \operatorname{BN}(50)$,

$1 \operatorname{BP}(50), \operatorname{BSEG}(50), \operatorname{CLONG}(5,10), \operatorname{CSHORT}(5,2), \operatorname{CUNIT}(5,2), \operatorname{DA}(50)$,

$2 \operatorname{DB}(50), \operatorname{DOSAT}(50), \operatorname{ENDC}(50,21), \operatorname{ENDL}(50,21), \operatorname{GEOM}(50,5)$,

$3 \operatorname{GLINC}(50,21), \operatorname{GLINL}(50,21), \operatorname{IGEOM}(50), \operatorname{IK} 2(50), \operatorname{K2}(50), \operatorname{KC}(50)$,

$4 \operatorname{KCR}(50), \operatorname{KORNR}(50), \operatorname{KORNF}(50), \operatorname{KNH} 4 R(50), \operatorname{KNH} 4 F(50), \operatorname{KNO} 2 R(50)$,

$5 \mathrm{KNO} 2 \mathrm{~F}(50), \mathrm{KNO} 3 \mathrm{R}(50), \mathrm{KPO} 4 \mathrm{~B}(50), \mathrm{KNCR} 1(50), \mathrm{KNCR} 2(50)$,

$7 \operatorname{NDIV}(50), \operatorname{OBSC}(200,21), \operatorname{OBSMI}(200), \operatorname{OBUNH} 3(200), \operatorname{PGEOM}(6000,5)$,

$8 \mathrm{PH}(50), \operatorname{PLOTC}(6000,30), \operatorname{PLOTL}(6000,30), \operatorname{PNET}(50), \operatorname{PRCOEF}(50)$,

$9 \operatorname{RESP}(50), \operatorname{RLINC}(50,21), \operatorname{RLINL}(50,21), \operatorname{SCOND}(50), \operatorname{SDESC}(50,11)$,

$\& \operatorname{SFPM}(50), \operatorname{SLOPE}(50), \operatorname{SNAME}(50,6), \operatorname{STARTC}(21), \operatorname{STARTL}(21)$,

\& TCONC(21), TEMP(50), TITLE1(19), TITLE2(19), TLOAD(21),

$\& \operatorname{TRINC}(50,21), \operatorname{TRINL}(50,21), \operatorname{TA}(50), \operatorname{TB}(50), \operatorname{TTRIBC}(50,21)$,

$\& \operatorname{TTRIBL}(50,21), \mathrm{UNH} 3(6000), \mathrm{VA}(50), \mathrm{VB}(50), \mathrm{WA}(50), \mathrm{WB}(50)$,

$\& \operatorname{XDIST}(6000), \operatorname{XLEN}(50), \operatorname{XSEG}(50), \operatorname{CTSVIG}(50)$

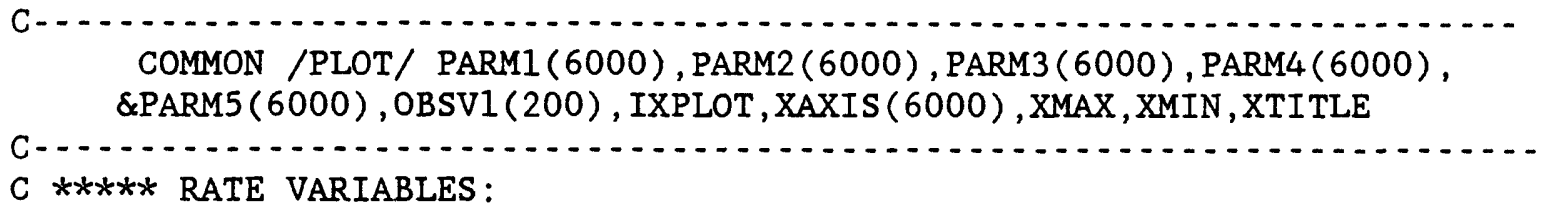

MAIN. .

MAIN. .

MAIN. .

MAIN. .

MAIN. .

MAIN. .

MAIN. .

MAIN. .

MAIN. .

MAIN. .

MAIN. .

MAIN. .

ROUTE.

ROUTE.

ROUTE.

ROUTE.

COMMON

COMMON

COMMON

COMMON

COMMON

COMMON

COMMON

COMMON

COMMON

COMMON

COMMON

COMMON

COMMON

COMMON

COMMON

COMMON

COMMON

COMMON

COMMON

COMMON

COMMON

COMMON

COMMON

COMMON

COMMON

COMMON

COMMON

COMMON

COMMON

COMMON

COMMON

COMMON

ROUTE.

ROUTE. 
APPENDIX I--LISTING OF COMPUTER CODE--Continued.

REAL K2T , KCT , KCRT, KORNRT, KORNFT, KNH4RT, KNH4FT, KNO2RT, KNO2FT, ROUTE.

\& KNO3RT , KPO4BT , KNCR1T, KNCR2T , K2TRM

$C * * * * *$

C ***** RATE ARRAYS:

C *****

REAL K2 , KCR , KC , KNR, KN , KORNR, KORNF, KNH4R, KNH4F, KNO2R, KNO2F, KNO3R, $\&$ KPO4B, KNCR1, KNCR2

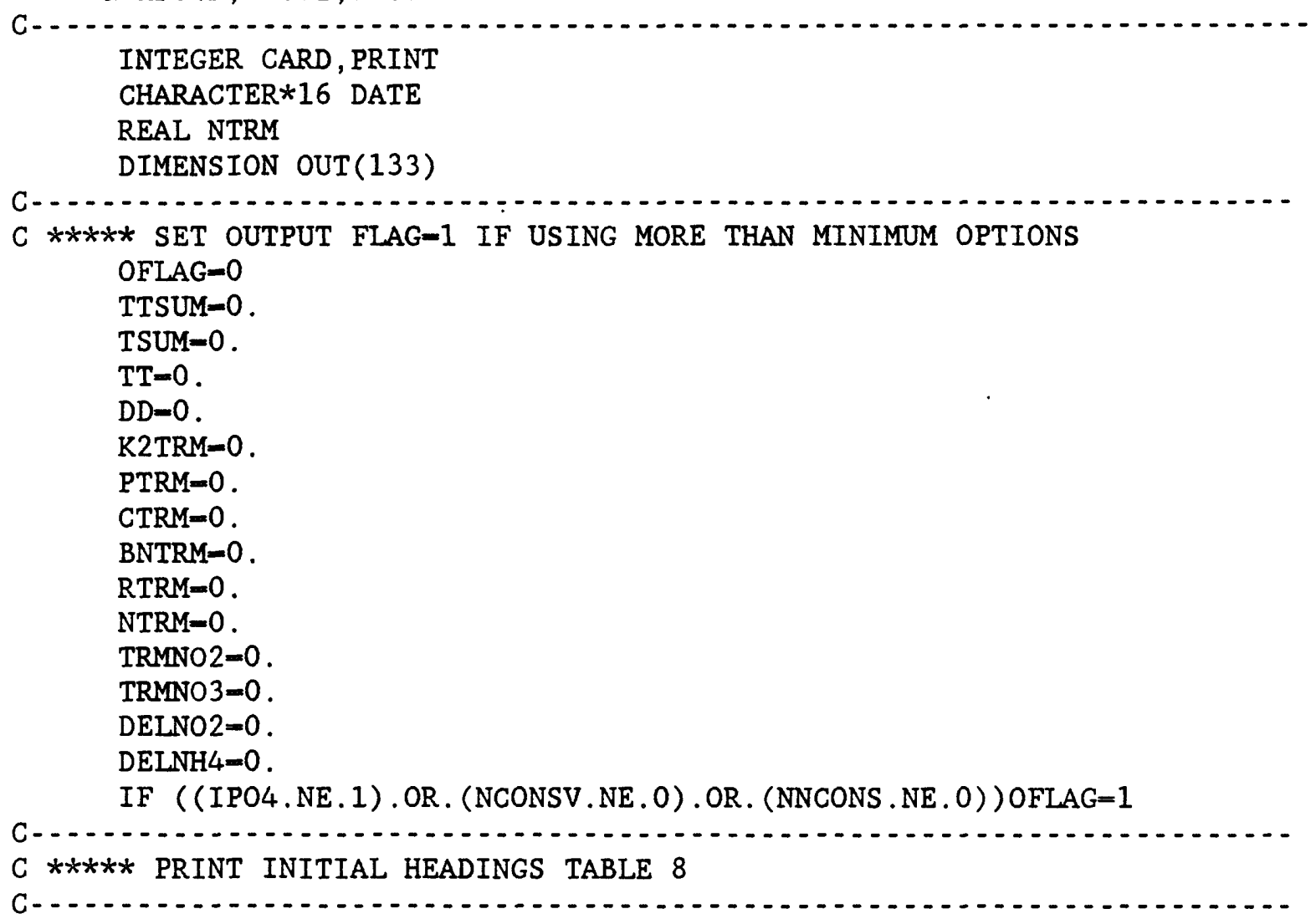

IF ((ITAB2.EQ.2).OR. (ITAB2.GT.6)) GOTO 60

WRITE (PRINT, 10) TITLE1, DATE, TITLE2

10 FORMAT (1H1,16X,'U.S. GEOLOGICAL SURVEY, WATER RESOURCES DIVISION'

\&, ': ', 2X, 'TRUCKEE RIVER WATER-QUALITY MODEL', 15X, '[REV 87.9]' ,

$\left.\& / 1 \mathrm{X}, 130\left({ }^{\prime}-^{\prime}\right), / 4 \mathrm{X}, / 20 \mathrm{X}, 19 \mathrm{~A} 4,10 \mathrm{X}, \mathrm{A} 16, / 20 \mathrm{X}, 19 \mathrm{~A} 4\right)$

WRITE (PRINT, 11)

11 FORMAT(/3OX, 'TABLE 8.--RESULTS OF COMPUTATIONS.' ,

$\& '(\text { RESULTS IN MG/L UNLESS OTHERWISE STATED. })^{\prime}, / 30 \mathrm{X}$,

$\left.\& 78\left({ }^{\prime}-{ }^{\prime}\right), /\right)$

IF (IMODEL. EQ. 1)WRITE (PRINT , 40)

40 FORMAT (36X, 'DISSOLVED-OXYGEN DEFICIT FACTORS', 30X, 'FINAL CONCENT', \&'RATIONS' $/ 27 \mathrm{X}, 62\left({ }^{\prime}-^{\prime}\right), 9 \mathrm{X}, 35\left(^{\prime}-^{\prime}\right) / 2 \mathrm{X},{ }^{\prime}$ RIVER DISCHARGE DAYS INI', *'TIAL REAER- CBOD NH4- NO2- PHOTO- RES - BEN- TOTAL', 4X, $\&$ SAT. FINAL ',7X, 'CBOD NH4-N NO2-N'/3X,'MILE',5X,'(CFS) T', \&'RAVEL DEF. ATION',11X,'NO2 NO3 SYNTH. PIR. THIC DEFI', $\&^{\prime} \mathrm{CIT}^{\prime}, 3 \mathrm{X},,^{\prime} \mathrm{D} .0 .^{\prime}, 3 \mathrm{X},,^{\prime} \mathrm{D} .0 .^{\prime}, 2 \mathrm{X},{ }^{\prime} \mathrm{SAT}^{\prime} / 1 \mathrm{X}, 3\left(1 \mathrm{X}, 7\left(^{\prime}=^{\prime}\right)\right), 1 \mathrm{X}$, $\left.\& 9\left(1 \mathrm{X}, 6\left({ }^{\prime}=^{\prime}\right)\right), 1 \mathrm{X}, 3\left(1 \mathrm{X}, 6\left(^{\prime}=^{\prime}\right)\right), 1 \mathrm{X}, 3\left(1 \mathrm{X}, 6\left(^{\prime}=^{\prime}\right)\right)\right)$

ROUTE.

ROUTE.

ROUTE.

ROUTE.

ROUTE.

ROUTE.

ROUTE.

ROUTE.

ROUTE.

ROUTE.

ROUTE.

ROUTE.

ROUTE.

ROUTE.

ROUTE.

ROUTE.

ROUTE.

ROUTE.

ROUTE.

ROUTE.

ROUTE.

ROUTE.

ROUTE.

ROUTE.

ROUTE.

ROUTE.

ROUTE.

ROUTE.

ROUTE.

ROUTE.

ROUTE.

ROUTE.

ROUTE.

ROUTE.

MAIN. .

MAIN. .

MAIN. .

ROUTE.

ROUTE.

ROUTE.

ROUTE.

ROUTE.

ROUTE.

ROUTE.

ROUTE.

ROUTE.

ROUTE.

ROUTE.

ROUTE. 
APPENDIX I--LISTING OF COMPUTER CODE--Continued.

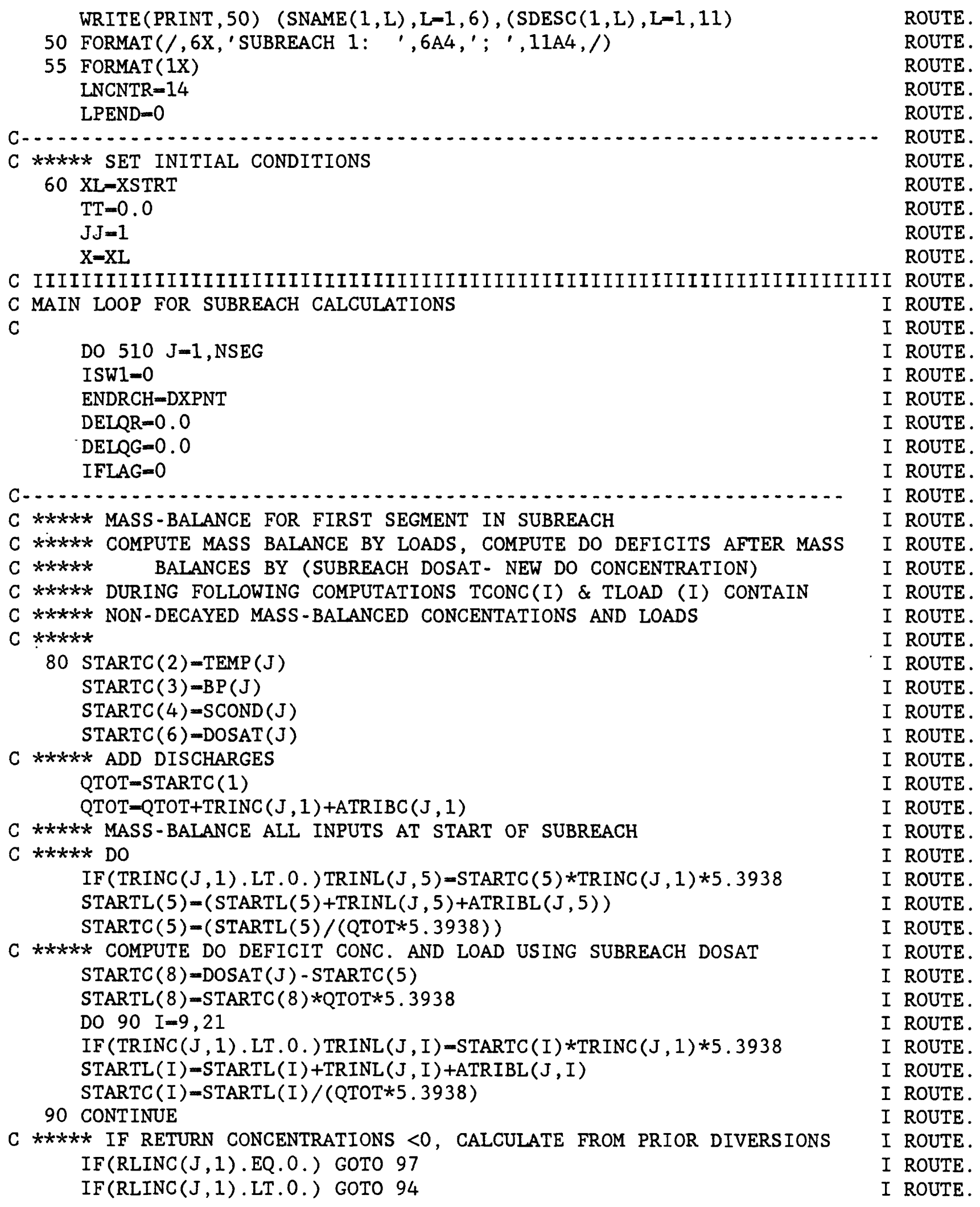


APPENDIX I--LISTING OF COMPUTER CODE--Continued.

$\operatorname{ISUB}=\operatorname{NDIV}(\mathrm{J})$

IF (ISUB.EQ.0) GOTO 92

C $* * * * *$ CALCULATE RETURN CONCENTRATIONS FROM UPSTEAM DIVERSION

DO $91 \mathrm{~K}=2,21$

IF (RLINC $(\mathrm{J}, \mathrm{K}) . \mathrm{GE} .0$.$) GOTO 91$

$\operatorname{RLINC}(\mathrm{J}, \mathrm{K})=\operatorname{RLINC}(\mathrm{J}, \mathrm{K}) * \operatorname{ENDC}(\operatorname{ISUB}, \mathrm{K}) *(-1$.

$\operatorname{RLINL}(\mathrm{J}, \mathrm{K})=\operatorname{RLINC}(\mathrm{J}, \mathrm{K}) * \operatorname{RLINC}(\mathrm{J}, 1) * 5.3938$

91 CONTINUE

GOTO 96

C $* * * * *$ CALCULATE CONCENTRATIONS FROM DIVERSIONS IN THIS SUBREACH

92 DO $93 \mathrm{~K}=2,21$

IF (RLINC (J,K).GE.0.) GOTO 93

$\operatorname{RLINC}(\mathrm{J}, \mathrm{K})-\operatorname{RLINC}(\mathrm{J}, \mathrm{K}) * \operatorname{STARTC}(\mathrm{K}) *(-1$.

$\operatorname{RLINL}(\mathrm{J}, \mathrm{K})=\operatorname{RLINC}(\mathrm{J}, \mathrm{K}) * \operatorname{RLINC}(\mathrm{J}, 1) * 5.3938$

93 CONTINUE

GOTO 96

$C * * * * *$ NEGATIVE FLOWS

94 DO $95 \mathrm{~K}-2,21$

$\operatorname{RLINC}(\mathrm{J}, \mathrm{K})-\operatorname{STARTC}(\mathrm{K})$

$\operatorname{RLINL}(\mathrm{J}, \mathrm{K})-\operatorname{RLINC}(\mathrm{J}, 1) * \operatorname{RLINC}(\mathrm{J}, \mathrm{K}) * 5.3938$

95 CONTINUE

96 CONTINUE

C ***** CALCULATE DOSAT AND RETURN DEFICIT IF NOT PREVIOUSLY DONE IF (RLINC $(J, 6)$. GT.0.) GOTO 97

CALL DOSATR (RLINC $(J, 2), \operatorname{BP}(J), \operatorname{RLINC}(J, 4), \operatorname{RLINC}(\mathrm{J}, 6))$

$\operatorname{RLINC}(J, 7)=((\operatorname{RLINC}(J, 5) / \operatorname{DOSAT}(J)) * 100$.

$\operatorname{RLINC}(J, 8)=\operatorname{RLINC}(J, 6)-\operatorname{RLINC}(J, 5)$

$\operatorname{RLINL}(\mathrm{J}, 8)=\operatorname{RLINC}(\mathrm{J}, 8) * \operatorname{RLINC}(\mathrm{J}, 1) * 5.3938$

97 CONTINUE

C $* * * * *$ NEGATIVE GROUND-WATER RETURNS

$\operatorname{IF}(\operatorname{GLINC}(\mathrm{J}, 1) . \mathrm{GE} .0$.$) GOTO 99$

DO $98 \mathrm{~K}=2,21$

$\operatorname{GLINC}(\mathrm{J}, \mathrm{K})=\operatorname{STARTC}(\mathrm{K})$

$98 \operatorname{GLINL}(\mathrm{J}, \mathrm{K})=\operatorname{GLINC}(\mathrm{J}, 1) * \operatorname{GLINC}(\mathrm{J}, \mathrm{K}) * 5.3938$

99 CONTINUE

C $* * * * *$ TEST FOR FIRST PASS

IF (ISW1.EQ.0)GOTO 140

C $* * * * *$ COMPUTE LINEAR RUNOFF INCREMENTS

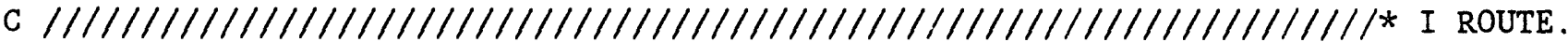

C $* * * * *$ RE-ENTRY POINT FOR SEGMENT CALCULATIONS WITHIN A SUBREACH

$\mathrm{C}$

100 CONTINUE

$C * * * * *$ CALCULATION LOOP FOR DXPNT INCREMENTS WITHIN SUBREACHES

C ***** CALCULATE DELTA Q TO ADD TO EACH INCREMENT IF(ILIN.EQ.0) GOTO 140

$\operatorname{IF}((\operatorname{RLINC}(\mathrm{J}, 1) \cdot \mathrm{EQ} \cdot 0$.$) .AND. (GLINC (\mathrm{J}, 1) \cdot \mathrm{EQ} \cdot 0)$.$) GOTO 140$ DELTLF=ENDRCH/XLEN $(J)$

$C * * * * *$ MASS-BALANCE LINEAR RUNOFF INPUTS

C $* * * * *$ MASS-BALANCE DO, THEN CALCULATE SUBREACH DEFICIT $\operatorname{IF}((\operatorname{ILIN} . E Q .1) . O R$. (ILIN.EQ.3)) DELQR=RLINC $(\mathrm{J}, 1) * D E L T L F$
I ROUTE.

I ROUTE.

I ROUTE.

I ROUTE.

I ROUTE.

I ROUTE.

I ROUTE.

I ROUTE.

I ROUTE.

I ROUTE.

I ROUTE.

I ROUTE.

I ROUTE.

I ROUTE.

I ROUTE.

I ROUTE.

I ROUTE.

I ROUTE.

I ROUTE.

I ROUTE.

I ROUTE.

I ROUTE.

I ROUTE.

I ROUTE.

I ROUTE.

I ROUTE.

I ROUTE.

I ROUTE.

I ROUTE.

I ROUTE.

I ROUTE.

I ROUTE.

I ROUTE.

I ROUTE.

I ROUTE.

I ROUTE.

I ROUTE.

* I ROUTE.

* I ROUTE.

* I ROUTE.

* I ROUTE.

* I ROUTE.

* I ROUTE.

* I ROUTE.

/* I ROUTE.

/* I ROUTE.

* I ROUTE.

/* I ROUTE.
I ROUTE. 
APPENDIX I--LISTING OF COMPUTER CODE--Continued.

IF (ILIN.GE. 2) DELQG=GLINC (J, 1)*DELTLF

C ****** PRO-RATE TOTAL SUBREACH INFLOW TO DXPNT

QTOT=QTOT + DELQR + DELQG

$\operatorname{IF}(D E L Q R . G T .0.) \operatorname{STARTL}(5)=\operatorname{STARTL}(5)+\operatorname{RLINL}(\mathrm{J}, 5) * \operatorname{DELTLF}$

IF (DELQR. IT .0) STARTL (5) $=\operatorname{STARTL}(5)+\operatorname{STARTC}(5) * D E L Q R * 5.3938$

IF (DELQG . GT . 0.) STARTL (5) $=\operatorname{STARTL}(5)+\operatorname{GLINL}(J, 5) * \operatorname{DELTLF}$

IF (DELQG . GT . 0.) STARTL ( 5) $=\operatorname{STARTL}(5)+\operatorname{STARTC}(5) * D E L Q G * 5.3938$

$\operatorname{STARTC}(5)=\operatorname{STARTL}(5) /(\mathrm{QTOT} * 5.3938)$

DO $130 \quad I=9,21$

IF (DELQR.GT.0.) $\operatorname{STARTL}(I)=\operatorname{STARTL}(I)+\operatorname{RLINL}(\mathrm{J}, \mathrm{I}) * \operatorname{DELTLF}$

IF (DELQG.GT.0.) STARTL(I) $=\operatorname{STARTL}(I)+\operatorname{GLINL}(J, I) * D E L T L F$

IF (DELQR. LT . 0.) STARTL (I) $=\operatorname{STARTL~(I)+STARTC~(I)~*DELQR*5.3938~}$

IF (DELQG . LT . O.) STARTL (I) $=\operatorname{STARTL~(I)~}+\operatorname{STARTC~(I)*DELQG*5.3938}$

$\operatorname{STARTC}(I)=\operatorname{STARTL}(I) /(\mathrm{QTOT} * 5.3938)$

130 CONTINUE

$\operatorname{STARTC}(8)=\operatorname{DOSAT}(\mathrm{J})-\operatorname{STARTC}(5)$

$\operatorname{STARTL}(8)=\operatorname{STARTC}(8) * \mathrm{QTOT} * 5.3938$

140 CONTINUE

C $* * * * *$ CHANNEL GEOMETRY FOR CALCULATION INTERVAL

C $* * * * *$ DEFAULT IS SEGMENT AVERAGE VALUES

DO $154 \mathrm{I}=1,5$

$154 \operatorname{PGEOM}(\mathrm{JJ}, \mathrm{I})=\operatorname{GEOM}(\mathrm{J}, \mathrm{I})$

$\mathrm{K} 2 \mathrm{~T}=\mathrm{K} 2(\mathrm{~J})$

IF(ISW1.EQ.0)GOTO 260

DELTT-DXPNT/(PGEOM (JJ , 1)*16.3636)

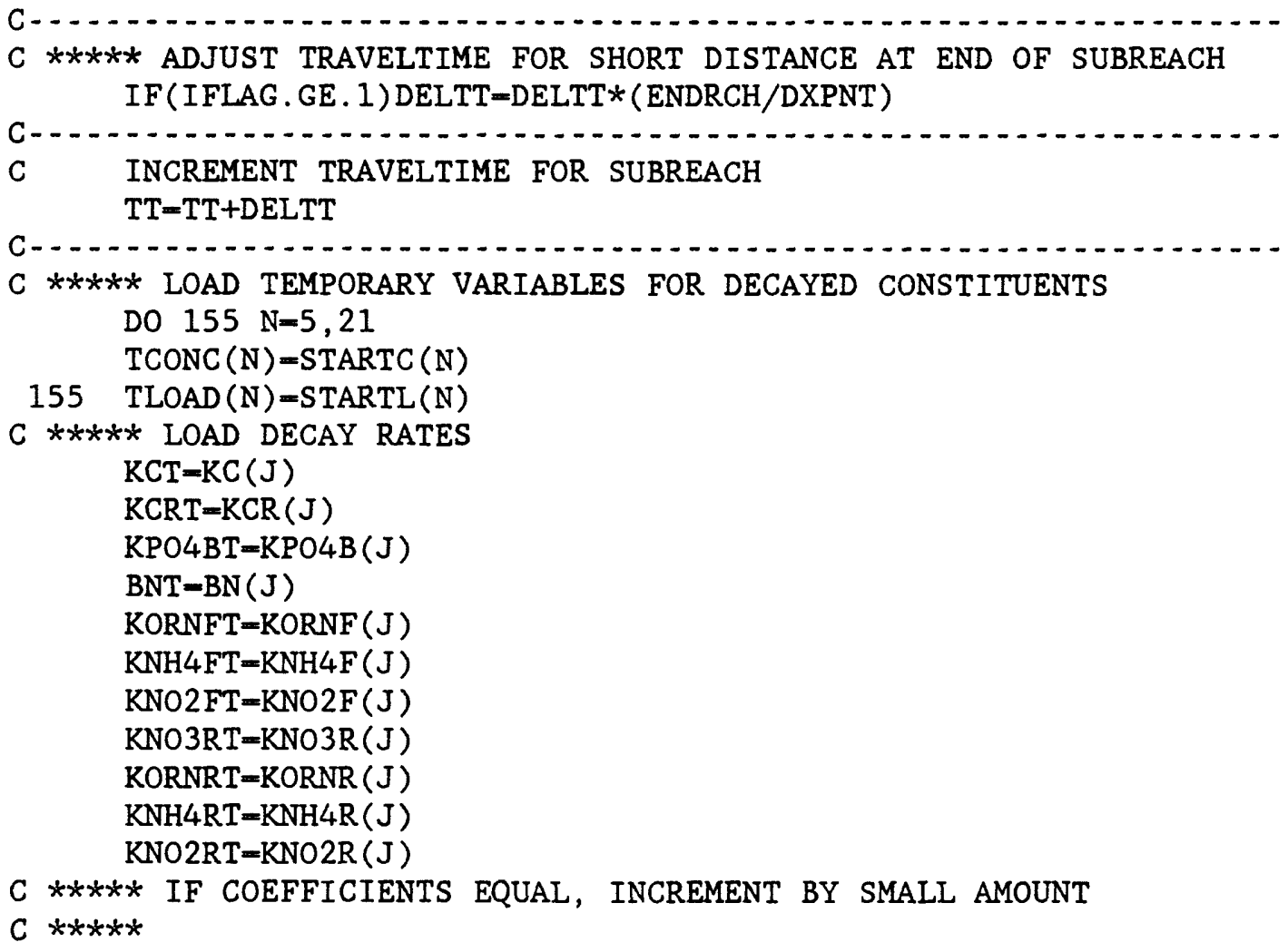

/* I ROUTE.

/* I ROUTE.

$/ *$ I ROUTE.

$/ *$ I ROUTE.

* I ROUTE.

* I ROUTE.

* I ROUTE.

* I ROUTE.

$/ *$ I ROUTE.

/* I ROUTE.

$/ *$ I ROUTE.

$/ *$ I ROUTE.

* I ROUTE.

* I ROUTE.

* I ROUTE.

$/ *$ I ROUTE.

* I ROUTE.

$/ *$ I ROUTE.

$/ *$ I ROUTE.

* I ROUTE.

$/ *$ I ROUTE.

* I ROUTE.

* I ROUTE.

* I ROUTE.

$/ *$ I ROUTE.

* I ROUTE.

* I ROUTE.

* I ROUTE.

* I ROUTE.

* I ROUTE.

$/ *$ I ROUTE.

* I ROUTE.

* I ROUTE.

/* I ROUTE.

* I ROUTE.

* I ROUTE.

* I ROUTE.

* I ROUTE.

/* I ROUTE.

$/ *$ I ROUTE.

/* I ROUTE.

/* I ROUTE.

* I ROUTE.

* I ROUTE.

$/ *$ I ROUTE.

* I ROUTE.

* I ROUTE.

$/ *$ I ROUTE.

* I ROUTE.

$/ *$ I ROUTE. 
APPENDIX I--LISTING OF COMPUTER CODE--Continued.

IF (K2T.EQ.KCRT) K2T $=\mathrm{K} 2 \mathrm{~T}+.00001$

IF (K2T.EQ.0.) $\mathrm{K} 2 \mathrm{~T}=.00001$

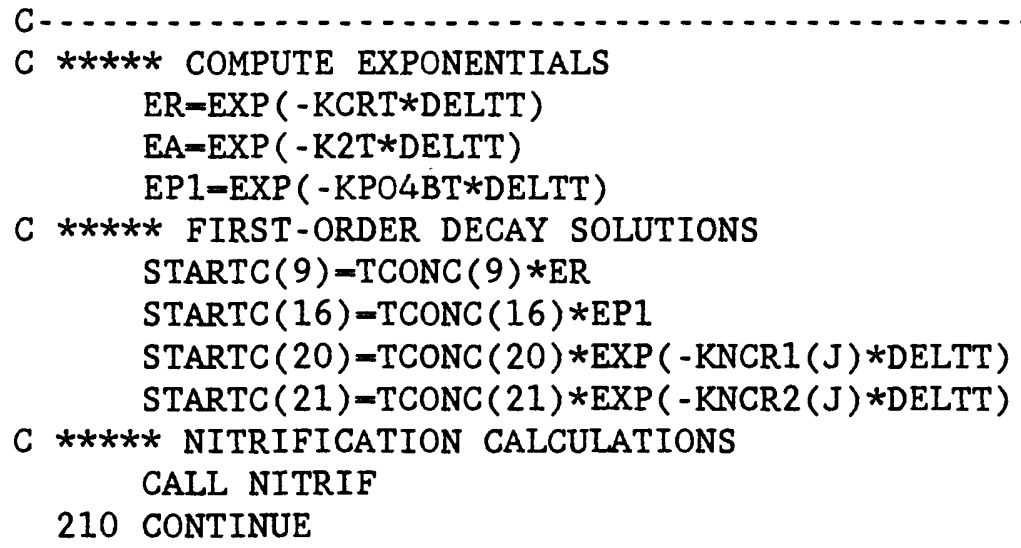

/* I ROUTE.

/* I ROUTE.

* I ROUTE.

/* I ROUTE.

* I ROUTE.

* I ROUTE.

* I ROUTE.

$/ *$ I ROUTE.

* I ROUTE.

/* I ROUTE.

/* I ROUTE.

/* I ROUTE.

* I ROUTE.

/* I ROUTE.

/* I ROUTE.

/* I ROUTE.

* I ROUTE.

* I I ROUTE.

/* I ROUTE.

* I I ROUTE.

* I I ROUTE.

* I ROUTE.

* I ROUTE.

* I ROUTE.

* I ROUTE.

* I ROUTE.

* I ROUTE.

* I ROUTE.

/* I ROUTE.

* I ROUTE.

/* I ROUTE.

* I ROUTE.

* I ROUTE.

* I ROUTE.

/* I ROUTE.

* I ROUTE.

* I ROUTE.

* I ROUTE.

* I I ROUTE.

* I ROUTE.

/* I ROUTE.

* I ROUTE.

* I ROUTE.

* I ROUTE.

* I ROUTE.

/* I ROUTE.

/* I ROUTE.

/* I ROUTE.

* I ROUTE.

/* I ROUTE. 
APPENDIX I--LISTING OF COMPUTER CODE--Continued.

C ****** SAVE RESULTS FOR NEXT NODE CALCULATION LOOP AND OUTPUT $\operatorname{PGEOM}(J J, 2)=$ TSUM + TT

PLOTC (JJ , 1) $=$ QTOT

$\operatorname{STARTC}(1)=Q T O T$

STARTC (15) $=\operatorname{STARTC}(11)+\operatorname{STARTC}(12)+\operatorname{STARTC}(13)+\operatorname{STARTC}(14)$

C ***** CALCULATE $\operatorname{STARTC}(7)=(\operatorname{STARTC}(5) / \operatorname{DOSAT}(\mathrm{J})) * 100$.

C $* * * * *$ RELOAD ALL FINAL VALUES

DO $270 \quad I=2,21$

$\operatorname{PLOTC}(J J, I)=\operatorname{STARTC}(I)$

$\operatorname{STARTL}(I)=\operatorname{STARTC}(I) * Q T O T * 5.3938$

PLOTL(JJ , I)-STARTL(I)

270 CONTINUE

$\operatorname{PLOTL}(\mathrm{JJ}, 1)=\mathrm{J}$

C ***** ESTIMATE UNIONIZED NH4 [THURSTON, RUSSO, \& EMERSON, 1974]

$\mathrm{C}$

IF (PH(J).EQ.0.) GOTO 275

PKA $=0.09018+2729.92 /(273.18+\mathrm{TEMP}(\mathrm{J}))$

IF (PH(J).EQ.0.) GOTO 275

FNH $4=1 . /(10 . * *(\mathrm{PKA}-\mathrm{PH}(\mathrm{J}))+1$.

$\mathrm{UNH} 3(\mathrm{JJ})=\operatorname{STARTC}(12) *$ FNH 4

275 CONTINUE

$\mathrm{C} * * * * *$

$\mathrm{C}$

C CALCULATE DIS N/P RATIO (MOLES/MOLE)

$\mathrm{C} * * * * *$

DIN=PLOTC (JJ , 12)+PLOTC (JJ , 13)+PLOTC (JJ , 14)

DINOP $=($ DIN $/ 14.007) /($ PLOTC $(J J, 20) / 30.974)$

PLOTC (JJ , 19) $=$ DINOP

C $* * * * *$ SAVE TRMS FOR DO BALANCE

C $* * * * *$ DO DEFICIT CONCENTRATIONS

PLOTC (JJ , 22) $=$ K2TRM

PLOTC (JJ , 23) $=$ CTRM

PLOTC (JJ , 24) $=$ NTRM

$\operatorname{PLOTC}(\mathrm{JJ}, 25)=$ TRMNO2

PLOTC (JJ , 26) $=$ TRMNO3

PLOTC $(J J, 27)=$ PTRM

PLOTC $(J J, 28)=$ RTRM

PLOTC $(J J, 29)=$ BNTRM

PLOTC (JJ , 30) $=\mathrm{K} 2 \mathrm{~T}$

C ****** DO DEFICIT TERMS

DO $280 \quad I=22,29$

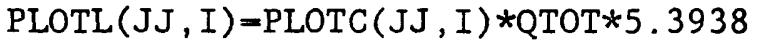

280 CONTINUE

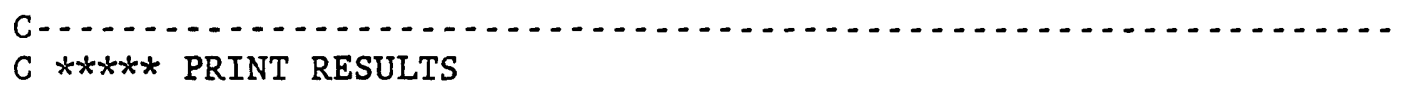

IF ((ITAB2. EQ.2). OR. (ITAB2.GT.6)) GOTO 490

C ****** PRINT N-CYCLE RESULTS: TABLE 8

$\mathrm{C}-$
/* I ROUTE.

* I ROUTE.

** I ROUTE.

* I ROUTE.

* I ROUTE.

/* I ROUTE.

/* I ROUTE.

/* I ROUTE.

$/ *$ I ROUTE.

** I ROUTE.

* I ROUTE.

$/ *$ I ROUTE.

/* I ROUTE.

** I ROUTE.

* I ROUTE.

/* I ROUTE.

* I ROUTE.

** I ROUTE.

** I ROUTE.

** I ROUTE.

** I ROUTE.

* I ROUTE.

** I ROUTE.

* I ROUTE.

/* I ROUTE.

/* I ROUTE.

/* I ROUTE.

* I ROUTE.

** I ROUTE.

* I ROUTE.

/* I ROUTE.

* I I ROUTE.

** I ROUTE.

** I ROUTE.

/* I ROUTE.

/* I ROUTE.

** I ROUTE.

* I ROUTE.

/* I ROUTE.

* I ROUTE.

* I ROUTE.

* I ROUTE.

* I ROUTE.

* I ROUTE.

* I ROUTE.

* I ROUTE.

/* I ROUTE.

/* I ROUTE.

* I ROUTE. 
APPENDIX I--LISTING OF COMPUTER CODE--Continued.

300 CONTINUE

IF(LNCNTR.LE.60) GOTO 305

WRITE(PRINT, 10) TITLE1, DATE, TITLE2

WRITE (PRINT, 11)

WRITE (PRINT, 40)

LNCNTR $=12$

LPEND $=0$

305 IF(ITAB2.LE. 1) GOTO 306

IF(IFLAG.EQ.0) GOTO 311

306 WRITE (PRINT, 310) XDIST(JJ), $\operatorname{PLOTC}(J J, 1), \operatorname{PGEOM}(J J, 2), \operatorname{TCONC}(8)$,

$\&(\operatorname{PLOTC}(\mathrm{JJ}, \mathrm{K}), \mathrm{K}=22,23),(\operatorname{PLOTC}(\mathrm{JJ}, \mathrm{K}), \mathrm{K}=25,29), \operatorname{PLOTC}(\mathrm{JJ}, 8)$,

$\& \operatorname{PLOTC}(J J, 6), \operatorname{PLOTC}(J J, 5), \operatorname{PLOTC}(J J, 7), \operatorname{PLOTC}(J J, 9)$,

$\&(\operatorname{PLOTC}(\mathrm{JJ}, \mathrm{K}), \mathrm{K}=12,13)$

310 FORMAT (1X, F8.2 , F8.1, F8 .2, 1X, 9F7.3, 1X, 2F7.2 , 2X, F5 .1, 1X, 3F7.2) LNCNTR=LNCNTR+1

311 CONTINUE

IF(IFLAG.EQ. 0) GOTO 490

C ****** N-CYCLE END-OF-SUBREACH

IF (ITAB2.LE. 1) WRITE (PRINT, 467)

467 FORMAT $\left(1 X, 132\left({ }^{\prime}-{ }^{\prime}\right)\right)$

420 WRITE (PRINT, 430) (PLOTL(JJ, K) , K=22, 23), (PLOTL(JJ , K) , K=25, 29), \& PLOTL (JJ , 8), PLOTL (JJ , 6), PLOTL(JJ , 5), PLOTL(JJ , 9) ,

$\&$ (PLOTL(JJ , K) , K=12, 13)

430 FORMAT ( $3 \mathrm{X}$, 'ENDING LOADS (LB/DAY):' , 8X, 8(F7.0), IX,

$\& 2(F 7.0), 8 \mathrm{X}, 3(\mathrm{~F} 7.0))$

IF (IFLAG.EQ.2) GOTO 490

IF (LNCNTR. LE . 60) GOTO 431

WRITE(PRINT, 10) TITLE1, DATE, TITLE2

WRITE (PRINT, 11)

WRITE (PRINT, 40)

LNCNTR $=12$

$431 \mathrm{JI}=\mathrm{J}+1$

WRITE (PRINT , 432) JI , ( $\operatorname{SNAME~(JI,~L)~,~L=1,~6),~(~} \operatorname{SDESC}(J \mathrm{I}, \mathrm{L})$

$\&, L=1,11$ )

432 FORMAT $\left(/, 6 \mathrm{X},{ }^{\prime}\right.$ SUBREACH ', I2,' : ', 6A4, ' ;', 11A4/)

LNCNTR=LNCNTR+5

490 CONTINUE
IF(IFLAG.NE. 2) JJ=JJ+1
IF (IFLAG.NE.0) GO TO 500
X-X-ABS (DXPNT)
XL=XL-DXPNT
XTEMP=XSEG(J) -XL
C***** TEST FOR SMALL INCREMENT AT END OF SUBREACH
ENDRCH=DXPNT -XTEMP
IF(ENDRCH.GT. (DXPNT+.O1)) ENDRCH=DXPNT
IF (XTEMP.GE. - .O1) XTEMP=0.0

/* I ROUTE.

* I ROUTE.

* I ROUTE.

/* I ROUTE.

* I ROUTE.

/* I ROUTE.

* I I ROUTE.

* I ROUTE.

* I ROUTE.

* I ROUTE.

/* I ROUTE.

* I ROUTE.

* I ROUTE.

* I ROUTE.

** I ROUTE.

$/ *$ I ROUTE.

$/ *$ I ROUTE.

* I ROUTE.

* I ROUTE.

* I ROUTE.

* I ROUTE.

* I ROUTE.

* I ROUTE.

* I ROUTE.

* I ROUTE.

* I ROUTE.

* I ROUTE.

* I ROUTE.

* I ROUTE.

* I ROUTE.

* I ROUTE.

* I ROUTE.

* I ROUTE.

* I ROUTE.

* I ROUTE.

** I ROUTE.

* I ROUTE.

$1 *$ I ROUTE.

* I ROUTE.

/* I ROUTE.

$/ *$ I ROUTE.

$/ *$ I ROUTE.

* I ROUTE.

$/ *$ I ROUTE.

* I ROUTE.

* I ROUTE.

$/ *$ I ROUTE.

$/ *$ I ROUTE.

$/ *$ I ROUTE.

$/ *$ I ROUTE. 
APPENDIX I--LISTING OF COMPUTER CODE--Continued.

C ****** SET UP DISTANCE FOR NEXT NODE $X T E M P=X T E M P * D X P N T / A B S(D X P N T)$

IF (XTEMP.LT. (0.0)) GO TO 100

$C * * * * *$ SET END-OF-SUBREACH FLAG

IFLAG $=1$

IF (J .EQ.NSEG) IFLAG $=2$

$\mathrm{X}=\mathrm{XSEG}(\mathrm{J})$

$X L=X S E G(J)$

C $* * * * *$ LOOP FOR NEXT CALCULATION INCREMENT

GO TO 100

/* I ROUTE.

/* I ROUTE.

/* I ROUTE.

/* I ROUTE.

/* I ROUTE.

/* I ROUTE.

/* I ROUTE.

/* I ROUTE.

/* I ROUTE.

$/ *$ I ROUTE.

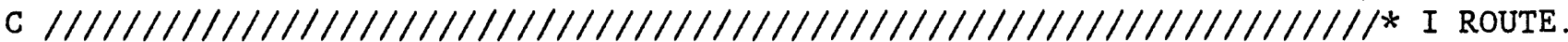

C $* * * * *$ END OF CALCULATIONS FOR A SUBREACH

500 IFLAG $=0$

$\mathrm{XL}=\mathrm{XSEG}(\mathrm{J})$

TSUM $=$ TSUM+TT

I ROUTE.

$\mathrm{TT}=0.0$

C ***** SAVE END-OF-SUBREACH CONCENTRATIONS AND LOADS

I ROUTE.

DO 505 II $=1,21$

$\operatorname{ENDC}(\mathrm{J}, \mathrm{II})=\operatorname{STARTC}(I I)$

I ROUTE.

I ROUTE.

I ROUTE.

I ROUTE.

I ROUTE.

$505 \operatorname{ENDL}(\mathrm{J}, \mathrm{II})=$ STARTL (II)

I ROUTE.

510 CONTINUE

I ROUTE.

$C * * * * *$ LOOP FOR NEXT SUBREACH

I ROUTE.

C IIIIIIIIIIIIIIIIIIIIIIIIIIIIIIIIIIIIIIIIIIIIIIIIIIIIIIIIIIIIIIIIIIIIII ROUTE. IF (JSEG.EQ.0) GO TO 522

C ***** STORE RESULTS IF AT END OF MAJOR TRIBUTARY

DO $520 \quad I=1,21$

$\operatorname{TTRIBC}(\mathrm{JSEG}, \mathrm{I})=\operatorname{STARTC}(I)$

TTRIBL (JSEG, I) $=$ STARTL (I)

520 CONTINUE

522 IF ((ITAB2.NE. 1).AND. (ITAB2.NE.6). AND. (ITAB2.NE. 9)) GOTO 525 RETURN

525 CONTINUE

C

C $* * * * *$ PRINT REMAINING OUTPUT TABLES (FULI AND SHORT TABLE 9)

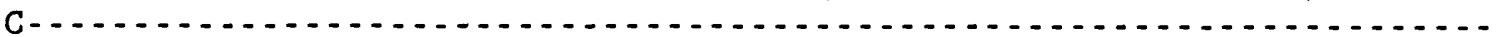

C ***** PRINT FULL TABLE 9

C ****** N-CYCLE

IF(JSEG.EQ. 0) WRITE (PRINT, 10) TITLE1, DATE, TITLE2

WRITE (PRINT , 536)

536 FORMAT(/30X, 'TABLE 9.--RESULTS OF COMPUTATIONS',

\&'FOR NITROGEN SPECIES AND OTHER CONSTITUENTS.' $\left., / 30 \mathrm{X}, 78\left({ }^{\prime}-^{\prime}\right)\right)$

ROUTE.

ROUTE.

ROUTE.

ROUTE.

ROUTE.

ROUTE.

ROUTE.

ROUTE.

ROUTE.

ROUTE.

ROUTE.

ROUTE.

ROUTE.

ROUTE.

ROUTE.

ROUTE.

ROUTE.

ROUTE.

WRITE (PRINT , 550) ((CSHORT ( I, K) , K=1, 2), I=4, 5), ( (CSHORT ( $I, K), K=1,2)$, $\& I=1,3),((\operatorname{CUNIT}(I, L), L=1,2), I=4,5),((\operatorname{CUNIT}(I, L), L=1,2), I=1,3)$

ROUTE.

ROUTE.

550 FORMAT (/33X, 'NITROGEN CYCLE' , 15X, 'UN-ION-' , 9X, 'NONCONSERVATIVES' , ROUTE.

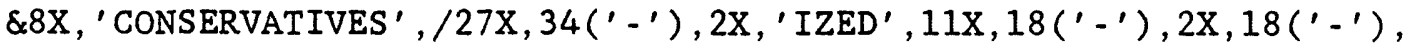
$\& / 2 \mathrm{X}$, 'RIVER', $2 \mathrm{X}$, ' DISCHARGE' ,

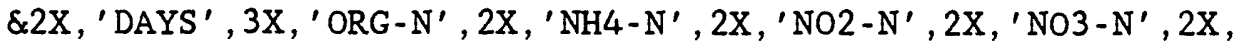

$\&^{\prime}$ TOT-N' $, 2 \mathrm{X},{ }^{\prime} \mathrm{NH} 3-\mathrm{N}^{\prime}, 2 \mathrm{X},{ }^{\prime} \mathrm{O}-\mathrm{P}-\mathrm{A}^{\prime}, 2 \mathrm{X}, 5(2 \mathrm{X}, 2 \mathrm{~A} 4)$, /2X, 'MILE' , 6X, ' (CFS)' , $\& 2 \mathrm{X}$, ' TRAVEL',

$\& 2 \mathrm{X}, 7\left({ }^{\prime}(\mathrm{MG} / \mathrm{L})^{\prime}, 1 \mathrm{X}\right), 5(2 \mathrm{X}, 2 \mathrm{~A} 4), / 1 \mathrm{X}, 7\left({ }^{\prime}=^{\prime}\right), 2 \mathrm{X}, 8\left({ }^{\prime}=^{\prime}\right), 2 \mathrm{X}, 8\left(6\left(^{\prime}==^{\prime}\right)\right.$,

ROUTE.

ROUTE.

ROUTE.

ROUTE.

ROUTE.

ROUTE. 
APPENDIX I--LISTING OF COMPUTER CODE--Continued.

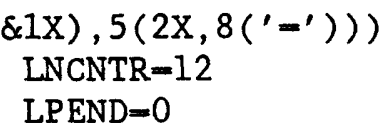

$C * * * * *$ FIND SUBREACH NUMBER AND WRITE SUBREACH HEADERS JOLD $=0$

DO $570 \quad I=1, \mathrm{JJ}$

$M=I F I X(\operatorname{PLOTL}(I, 1))$

IF(M.EQ.JOLD) GOTO 555

WRITE (PRINT , 432) M, ( $\operatorname{SNAME~}(M, L), L=1,6)$,

$\&(\operatorname{SDESC}(M, L), L=1,11)$

555 JOLD $=M$

IF ((ITAB2.EQ.0). OR. (ITAB2.EQ.2)) GOTO 558

IF ( (I.NE.JJ).AND. (PLOTL ( (I+1),1).EQ.PLOTL $(I, 1)))$ GOTO 565

558 WRITE(PRINT , 560) XDIST (I), $\operatorname{PLOTC}(I, 1), \operatorname{PGEOM}(I, 2),(\operatorname{PLOTC}(I, K), K=11,15$

\&) , UNH3 (I) , PLOTC ( I , 16), (PLOTC ( I , K) , K=20, 21), (PLOTC ( I , K) , K=17, 19)

560 FORMAT (1X,F7.2, 1X, F9.2,6(2X, F5.2) , 2X, F5.3, 2X, F5.2, 2X, 5F10.2)

LNCNTR-LNCNTR+1

IF ( (I.NE.JJ).AND. (PLOTL( (I+1),1).EQ.PLOTL $(I, 1))$ ) GOTO 565

IF (ITAB2.LE.2) WRITE(PRINT, 467)

IF( (I.EQ.JJ) .OR. (PLOTL( (I+1), 1).NE.PLOTL(I, 1))) WRITE(PRINT, 563)

$\&(\operatorname{PLOTL}(I, K), K=11,15), \operatorname{PLOTL}(I, 16),(\operatorname{PLOTL}(I, K), K=20,21)$,

$\&(\operatorname{PLOTL}(I, K), \mathrm{K}=17,19)$

563 FORMAT ( $3 \mathrm{X}$, 'ENDING LOADS (LB/DAY):', 5F7.0,7X, F7.0, 2X, 5F10.0)

LNCNTR=LNCNTR+5

565 IF(LNCNTR. GE. 60) LPEND=1

IF(LPEND.EQ.0) GOTO 570

IF(I.EQ.JJ) GOTO 570

WRITE(PRINT, 10) TITLE1, DATE, TITLE2

WRITE (PRINT, 536)

WRITE (PRINT , 550) ((CSHORT ( $L, K), K=1,2), \mathrm{L}=4,5),((\operatorname{CSHORT}(\mathrm{L}, \mathrm{K}), \mathrm{K}=1,2$ ),

$\& L=1,3),((\operatorname{CUNIT}(L, K), K=1,2), L=4,5),((\operatorname{CUNIT}(L, K), K=1,2), L=1,3)$

LNCNTR $=12$

LPEND $=0$

570 CONTINUE

700 RETURN

C $* * * * * *$

END

\section{$\mathrm{C}$ \\ C \\ C}

SUBROUTINE NITRIF

C $* * * * *$ SUBROUTINE TO PERFORM CALCULATIONS FOR DECAY OF INDIVIDUAL

C $* * * * * *$ NITROGEN SPECIES

C $* x * * *$ VARIABLES COMMON TO ONE OR MORE OF MAIN, READCD, ROUTE, NITRIF,

C $* * * * * * *$ SUBROUTINES

C $* * * * * * x$ SCALARS :

$\mathrm{C} * * * * * *$

COMMON /VALL/ BDN, BNT, CARD, DELNH4, DELNO2, DELTT, DXPNT, EA,

1 ER, ICDBUF, IEND, IGRAPH, ITYPE, ILIN, IMODEL, INITSW, IPLOT,

ROUTE.

ROUTE.

ROUTE.

ROUTE.

ROUTE.

ROUTE.

ROUTE.

ROUTE.

ROUTE.

ROUTE.

ROUTE.

ROUTE :

ROUTE.

ROUTE.

ROUTE.

ROUTE.

ROUTE.

ROUTE.

ROUTE.

ROUTE.

ROUTE.

ROUTE.

ROUTE.

ROUTE.

ROUTE.

ROUTE.

ROUTE.

ROUTE.

ROUTE.

ROUTE.

ROUTE.

ROUTE.

ROUTE.

ROUTE.

ROUTE.

ROUTE.

ROUTE.

ROUTE.

NITRIF

NITRIF

NITRIF

NITRIF

NITRIF

COMMON

COMMON

COMMON

COMMON

COMMON

COMMON

COMMON 
APPENDIX I--LISTING OF COMPUTER CODE--Continued.

2 IPO4, IPRMIN, IPNET, IRET, ISW1, ISWBAD, ITAB1, ITAB2, ITEST,

COMMON

3 ITFLAG, IXGEOM, J, JJ , JSEG, KPLOT, K2T, KCT, KCRT, KORNRT,

COMMON

4 KORNFT, KNH4RT, KNH4FT, KNO2RT, KNO2FT, KNO3RT, KP04BT, KNCRIT,

5 KNCR2T, LNCNTR, MPT, NCONSV, NNCONS, NSEG, PRINT, QTOT, TT,

6 TTSUM, XEND, XL, XSTRT, IRESP, DATE

\section{C $* * * * * * x$}

C $* * * * * *$ ARRAYS:

$C * * * * * * x$

COMMON /ALL/ $\operatorname{AA}(50), \operatorname{AB}(50), \operatorname{ATRIBC}(50,21), \operatorname{ATRIBL}(50,21), \operatorname{BN}(50)$,

$1 \operatorname{BP}(50), \operatorname{BSEG}(50), \operatorname{CLONG}(5,10), \operatorname{CSHORT}(5,2), \operatorname{CUNIT}(5,2), \operatorname{DA}(50)$,

$2 \operatorname{DB}(50), \operatorname{DOSAT}(50), \operatorname{ENDC}(50,21), \operatorname{ENDL}(50,21), \operatorname{GEOM}(50,5)$,

$3 \operatorname{GLINC}(50,21), \operatorname{GLINL}(50,21), \operatorname{IGEOM}(50), \operatorname{IK2}(50), \mathrm{K} 2(50), \mathrm{KC}(50)$,

$4 \operatorname{KCR}(50), \operatorname{KORNR}(50), \operatorname{KORNF}(50), \operatorname{KNH} 4 R(50), \operatorname{KNH} 4 F(50), \operatorname{KNO} 2 R(50)$,

$5 \mathrm{KNO} 2 \mathrm{~F}(50), \operatorname{KNO} 3 \mathrm{R}(50), \mathrm{KPO} 4 \mathrm{~B}(50), \mathrm{KNCR} 1(50), \operatorname{KNCR2}(50)$,

$7 \operatorname{NDIV}(50), \operatorname{OBSC}(200,21), \operatorname{OBSMI}(200), \operatorname{OBUNH} 3(200), \operatorname{PGEOM}(6000,5)$,

$8 \mathrm{PH}(50), \operatorname{PLOTC}(6000,30), \operatorname{PLOTL}(6000,30), \operatorname{PNET}(50), \operatorname{PRCOEF}(50)$,

$9 \operatorname{RESP}(50), \operatorname{RLINC}(50,21), \operatorname{RLINL}(50,21), \operatorname{SCOND}(50), \operatorname{SDESC}(50,11)$,

$\& \operatorname{SFPM}(50), \operatorname{SLOPE}(50), \operatorname{SNAME}(50,6), \operatorname{STARTC}(21), \operatorname{STARTL}(21)$,

\& TCONC(21), TEMP(50), $\operatorname{TITLE} 1(19), \operatorname{TITLE} 2(19), \operatorname{TLOAD}(21)$,

$\& \operatorname{TRINC}(50,21), \operatorname{TRINL}(50,21), \operatorname{TA}(50), \operatorname{TB}(50), \operatorname{TTRIBC}(50,21)$,

$\& \operatorname{TTRIBL}(50,21), \mathrm{UNH} 3(6000), \mathrm{VA}(50), \mathrm{VB}(50), \mathrm{WA}(50), \mathrm{WB}(50)$,

$\& \operatorname{XDIST}(6000), \operatorname{XLEN}(50), \operatorname{XSEG}(50), \operatorname{CTSVIG}(50)$

$$
\begin{aligned}
& \text { C } \\
& \text { C } \\
& \text { C }
\end{aligned}
$$

COMMON /PLOT/ PARM1 (6000), PARM2 (6000), PARM3 (6000), PARM4 (6000),

\&PARM5 (6000), OBSV1 (200), IXPLOT, XAXIS (6000), XMAX, XMIN , XTITLE

C $* * * * *$ RATE VARIABLES:

REAL K2T , KCT , KCRT , KORNRT , KORNFT, KNH4RT, KNH4FT , KNO2RT , KNO2FT,

$\mathrm{C} * * * * * * *$

\& KNO3RT , KPO4BT , KNCR1T , KNCR2T , K2TRM

C ****** RATE ARRAYS:

$C * * * * *$

REAL K2, KCR , KC, KNR , KN, KORNR, KORNF, KNH4R, KNH4F, KNO2R, KNO2F, KNO3R, \& $\mathrm{KPO} 4 \mathrm{~B}, \mathrm{KNCR} 1, \mathrm{KNCR} 2$

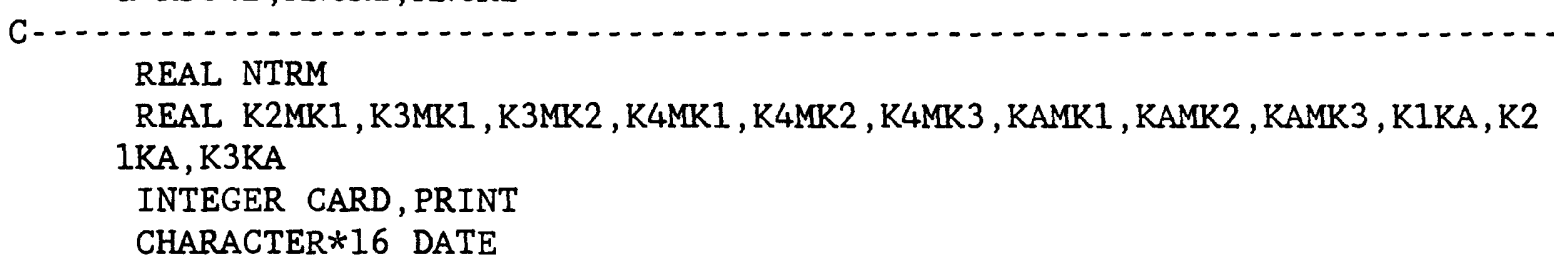

$C * * * * * x$

DATA END/1HE/

C *******

C NITRIFICATION CALCULATIONS REPROGRAMMED JAN 26, 1979

C N1 = STARTC (11)

C N2 $=$ STARTC (12)

$\mathrm{N} 10=\mathrm{TCONC}(11)$

$\mathrm{K} 11=\mathrm{KORNRT}$

K12 $=$ KORNFT

C N3 $=\operatorname{STARTC}(13)$

$\mathrm{N} 20=\mathrm{TCONC}(12)$

$\mathrm{K} 22=\mathrm{KNH} 4 \mathrm{RT}$

$\mathrm{K} 23=\mathrm{KNH} 4 \mathrm{FT}$

$\mathrm{N} 30=\mathrm{TCONC}(13)$

$\mathrm{K} 33=\mathrm{KNO} 2 \mathrm{RT}$

$\mathrm{K} 34=\mathrm{KNO} 2 \mathrm{FT}$

C *******

$\mathrm{N} 40=\operatorname{TCONC}(14)$

$\mathrm{K} 44=\mathrm{KNO3RT}$

COMMON

COMMON

COMMON

COMMON

COMMON

COMMON

COMMON

COMMON

COMMON

COMMON

COMMON

COMMON

COMMON

COMMON

COMMON

COMMON

COMMON

COMMON

COMMON

COMMON

COMMON

COMMON

COMMON

COMMON

NITRIF

NITRIF

NITRIF

NITRIF

NITRIF

NITRIF

NITRIF

NITRIF

NITRIF

NITRIF

NITRIF

NITRIF

NITRIF

NITRIF

NITRIF

NITRIF

NITRIF

NITRIF

NITRIF

NITRIF

NITRIF

NITRIF

NITRIF

K2MK $1=$ KNH 4 RT - KORNRT

NITRIF 
APPENDIX I--IISTING OF COMPUTER CODE--Continued.

K3MK1 $=$ KNO2RT - KORNRT

NITRIF

$\mathrm{K} 3 \mathrm{MK} 2=\mathrm{KNO} 2 \mathrm{RT}-\mathrm{KNH} 4 \mathrm{RT}$

NITRIF

K4MK1 $=$ KNO3RT - KORNRT

NITRIF

K4MK2 $=$ KNO3RT - KNH4RT

NITRIF

$\mathrm{K} 4 \mathrm{MK} 3=\mathrm{KNO} 3 \mathrm{RT}-\mathrm{KNO} 2 \mathrm{RT}$

NITRIF

$C * * * * *$ SET DIFFERENCE TERMS TO NON-ZERO VALUES

NITRIF

IF (K2MK1.EQ.0.) $\mathrm{K} 2 \mathrm{MK} 1=.002$

NITRIF

IF (K3MK1.EQ.0.) $\mathrm{K} 3 \mathrm{MK} 1=.002$

NITRIF

IF (K3MK2.EQ.0.) $\mathrm{K} 3 \mathrm{MK} 2=.001$

NITRIF

IF (K4MK1.EQ.0.) $\mathrm{K} 4 \mathrm{MK} 1=.002$

NITRIF

IF (K4MK2.EQ.0.) $\mathrm{K} 4 \mathrm{MK} 2=.001$

NITRIF

C

IF (K4MK3.EQ.0.) $\mathrm{K} 4 \mathrm{MK} 3=.001$

NITRIF

NITRIF

$\mathrm{A}=\mathrm{KORNFT} * \mathrm{TCONC}(11) / \mathrm{K} 2 \mathrm{MK} 1$

$\mathrm{B}=\mathrm{TCONC}(12)-\mathrm{A}$

$\mathrm{C}=\mathrm{A} * \mathrm{KNH} 4 \mathrm{FT} / \mathrm{K} 3 \mathrm{MK} 1$

$\mathrm{D}=\mathrm{TCONC}(12) * \mathrm{KNH} 4 \mathrm{FT} / \mathrm{K} 3 \mathrm{MK} 2-\mathrm{A} * \mathrm{KNH} 4 \mathrm{FT} / \mathrm{K} 3 \mathrm{MK} 2$

$\mathrm{E}=\mathrm{TCONC}(13)-\mathrm{TCONC}(12) \times \mathrm{KNH} 4 \mathrm{FT} / \mathrm{K} 3 \mathrm{MK} 2-\mathrm{C}+\mathrm{A} * \mathrm{KNH} 4 \mathrm{FT} / \mathrm{K} 3 \mathrm{MK} 2$

$\mathrm{F}=\mathrm{C} * \mathrm{KNO} 2 \mathrm{FT} / \mathrm{K} 4 \mathrm{MK} 1$

$\mathrm{G}=\mathrm{KNH} 4 \mathrm{FT} * \mathrm{KNO} 2 \mathrm{FT} * \mathrm{~B} /(\mathrm{K} 3 \mathrm{MK} 2 * \mathrm{~K} 4 \mathrm{MK} 2)$

$\mathrm{H}=-\mathrm{KNO} 2 \mathrm{FT} / \mathrm{K} 4 \mathrm{MK} 3 *(-\mathrm{TCONC}(13)+\mathrm{C}+\mathrm{KNH} 4 \mathrm{FT} / \mathrm{K} 3 \mathrm{MK} 2 * \mathrm{~B})$

C $* * * * * *$

$\mathrm{A} 1=\mathrm{TCONC}(14)-\mathrm{F}-\mathrm{G}-\mathrm{H}$

NITRIF

NITRIF

NITRIF

NITRIF

NITRIF

NITRIF

NITRIF

NITRIF

NITRIF

NITRIF

$E 1=\operatorname{EXP}(-$ KORNRT $*$ DELTT $)$

$E 2=\operatorname{EXP}(-\mathrm{KNH} 4 \mathrm{RT} * \mathrm{DELTT})$

$E 3=\operatorname{EXP}(-\mathrm{KNO} 2 \mathrm{RT} * \mathrm{DELTT})$

C $* * * * * *$

E4 $=\operatorname{EXP}(-\mathrm{KNO} 3 \mathrm{RT} * \mathrm{DELTT})$

NITRIF

NITRIF

NITRIF

NITRIF

NITRIF

C NITROGEN COMPONENTS CONCENTRATIONS

C *******

$\operatorname{STARTC}(11)=\operatorname{TCONC}(11) * E 1$

$\operatorname{STARTC}(12)=A * E 1+B * E 2$

$\operatorname{STARTC}(13)=\mathrm{C} * \mathrm{E} 1+\mathrm{D} * \mathrm{E} 2+\mathrm{E} * \mathrm{E} 3$

$\operatorname{STARTC}(14)=\mathrm{F} * \mathrm{E} 1+\mathrm{G} * \mathrm{E} 2+\mathrm{H} * \mathrm{E} 3+\mathrm{A} 1 * \mathrm{E} 4$

C $* * * * * * x$

C NITRIFICATION DO DEFICIT

C *******

$\mathrm{EA}=\operatorname{EXP}(-\mathrm{K} 2 \mathrm{~T} * \mathrm{DELTT})$

KAMK $1=\mathrm{K} 2 \mathrm{~T}-\mathrm{KORNRT}$

KAMR2=K2T $-\mathrm{KNH} 4 \mathrm{RT}$

KAMK3 $=\mathrm{K} 2 \mathrm{~T}-\mathrm{KNO} 2 \mathrm{RT}$

IF (KAMK1.EQ.0.) KAMK1=.002

IF (KAMK2.EQ.0.) KAMK2 $=.001$

IF (KAMK3.EQ.0.) KAMK3 $=.001$

$\mathrm{K} 1 \mathrm{KA}=(\mathrm{E} 1-\mathrm{EA}) / \mathrm{KAMK1}$

$\mathrm{K} 2 \mathrm{KA}=(\mathrm{E} 2-\mathrm{EA}) / \mathrm{KAMK} 2$

$\mathrm{K} 3 \mathrm{KA}=(\mathrm{E} 3-\mathrm{EA}) / \mathrm{KAMK} 3$

$C * * * * * * x$

DELNH4 $=\mathrm{KNH} 4 \mathrm{FT} *(\mathrm{~A} * \mathrm{~K} 1 \mathrm{KA}+\mathrm{B} * \mathrm{~K} 2 \mathrm{KA})$

DELNO2 $=\mathrm{KNO} 2 \mathrm{FT} *(\mathrm{C} * \mathrm{~K} 1 \mathrm{KA}+\mathrm{D} * \mathrm{~K} 2 \mathrm{KA}+\mathrm{E} * \mathrm{~K} 3 \mathrm{KA})$

NITRIF

NITRIF

NITRIF

NITRIF

NITRIF

NITRIF

NITRIF

NITRIF

NITRIF

NITRIF

NITRIF

NITRIF

NITRIF

NITRIF

NITRIF

NITRIF

NITRIF

NITRIF

NITRIF

NITRIF

NITRIF

NITRIF 
APPENDIX I--LISTING OF COMPUTER CODE--Continued.

C TEST FOR VALUES .LT. ZERO

IF (STARTC(14).LT. (0.0)) $\operatorname{STARTC}(14)=0.0$

IF (STARTC (13).LT. (0.0)) $\operatorname{STARTC}(13)=0.0$

IF (STARTC(12).LT. (0.0)) $\operatorname{STARTC}(12)=0.0$

IF (STARTC(11).LT. (0.0)) $\operatorname{STARTC}(11)=0.0$

RETURN

END

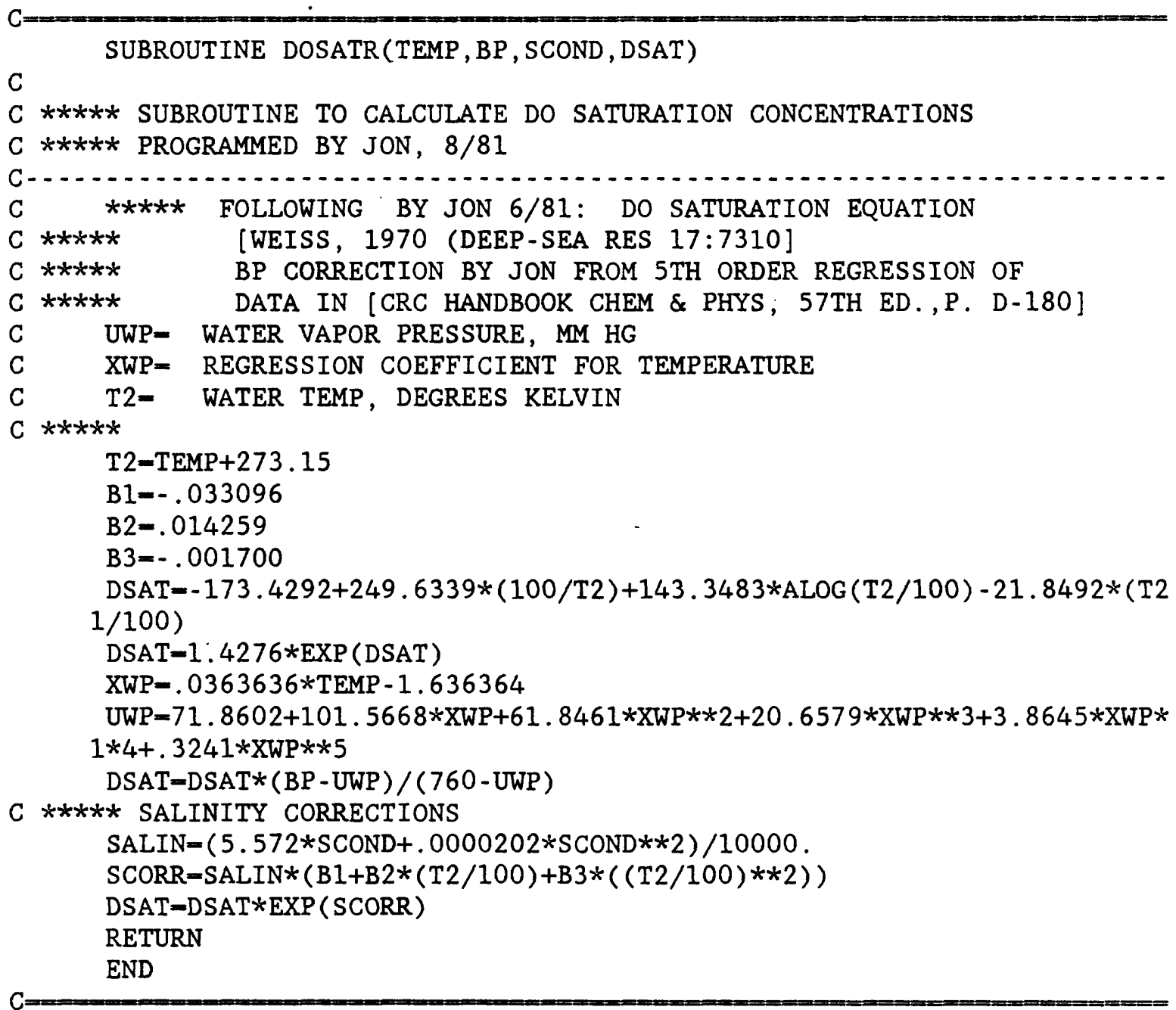

SUBROUTINE CGEOM(NSEG, VEL, TTIME, AREA, DEPTH, WIDTH, VA , VB, TA , TB , AA, $1 \mathrm{AB}, \mathrm{DA}, \mathrm{DB}, \mathrm{WA}, \mathrm{WB}$, IGEOM ,, , XLEN)

$\mathrm{C}$

C $* * * * *$ CHANNEL GEOMETRY COMPUTATIONS

C $* * * * * *$ PROGRAMMED BY JON, 12/81

C $* * * * * *$

C ****** INPUT DATA FOR NORMAL CALCULATIONS:

C $* * * x *$

C $* * x+x$

C $* * * * *$

C $* * * * *$

\begin{tabular}{clc} 
IGEOM & INPUT DATA & IGEOM \\
\hline 1 & VEL, DEPTH & 4 \\
2 & TTIME, DEPTH & 5
\end{tabular}

INPUT DATA

VEL, WIDTH TTIME, WITH

NITRIF

NITRIF

NITRIF

NITRIF

NITRIF

NITRIF

NITRIF

DOSATR

DOSATR

DOSATR

DOSATR

DOSATR

DOSATR

DOSATR

DOSATR

DOSATR

DOSATR

DOSATR

DOSATR

DOSATR

DOSATR

DOSATR

DOSATR

DOSATR

DOSATR

DOSATR

DOSATR

DOSATR

DOSATR

DOSATR

DOSATR

DOSATR

DOSATR

DOSATR

DOSATR

DOSATR

DOSATR

DOSATR

CGEOM.

CGEOM.

CGEOM.

CGEOM.

CGEOM.

CGEOM.

CGEOM.

CGEOM.

CGEOM.

CGEOM.

CGEOM.

CGEOM. 
APPENDIX I--LISTING OF COMPUTER CODE--Continued.

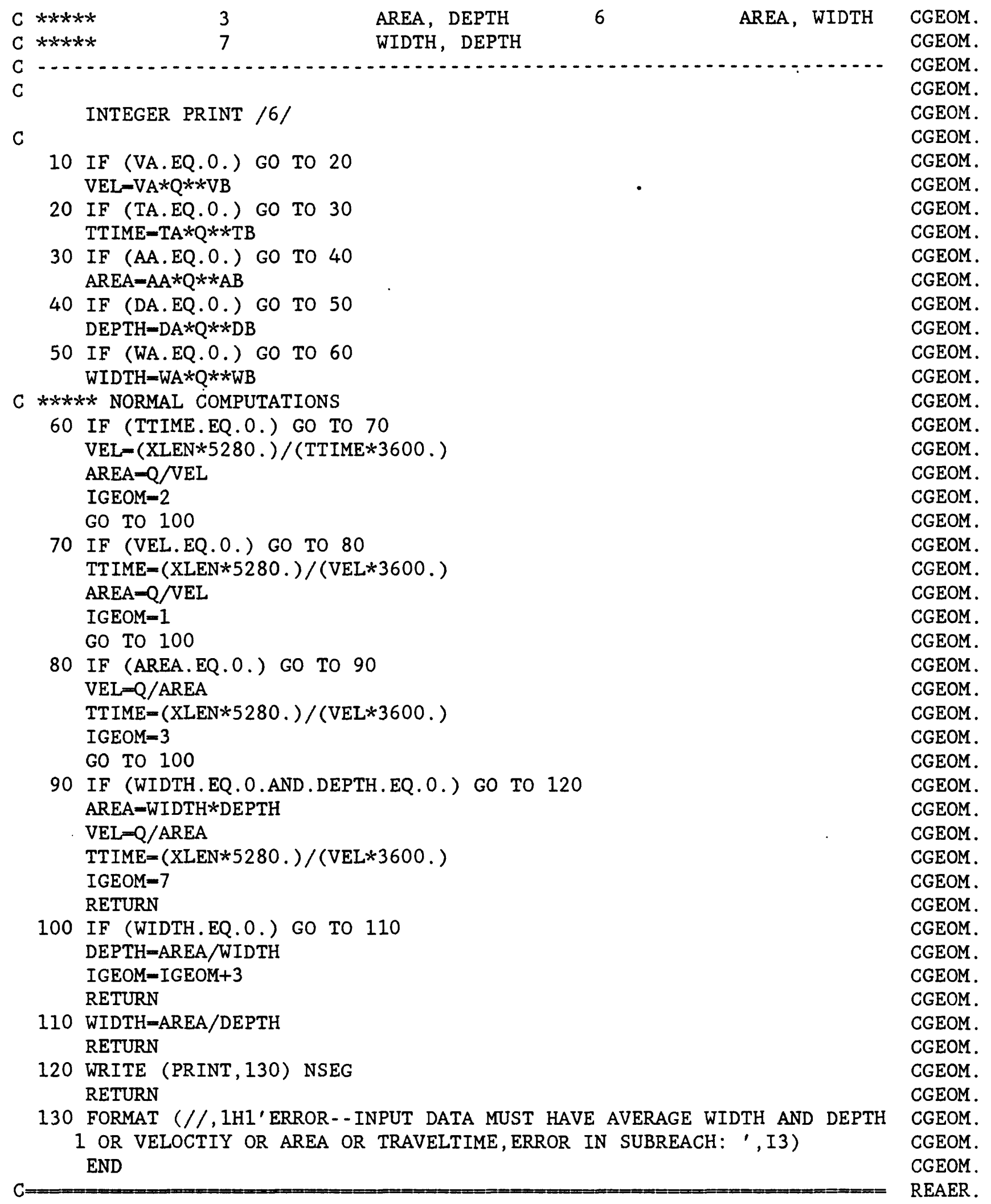


APPENDIX I--LISTING OF COMPUTER CODE--Continued.

SUBROUTINE REAER (NEQUA, DEP, U, S, XK2, CTSV)

REAER.

C

C $* * * * * *$ PROGRAMMED BY LARRY SMITH, 1980

C ****** MODIFIED BY JON NOWLIN, $6 / 81,9 / 83$

$\mathrm{C}$

C DEP $=$ DEPTH, $Q=$ DISCHARGE, AR= AREA, DT $=$ TEMP $-20, S=S L O P E$,

C XK2 = REAERATION COEF, CTSV $=$ TSV. ESCAPE COEFFICIENT, NEQUA= EQUATION C FT CFS FT*2 DEG-C PURE PER DAY

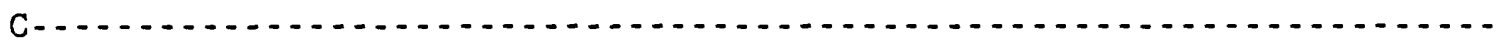

$\mathrm{C}$

$\mathrm{C} * * * * * *$

DATA PI/3.1415927/

C $* * * * *$

Go TO $(10,20,50,60,70,80,90,100,900,1000,1100,1200)$, NEQUA

C ***** [BENNETT \& RATHBUN, 1972]--EQUATION FROM FIELD DATA

C $* * * * * *$

$10 \mathrm{XK} 2=20.2 *(U * * .607) /(\mathrm{DEP} * * 1.689)$ RETURN

C $* * * * * * *$

C ***** [VELZ, 1970], FORMULATION: [HIRSCH, 1981],

C ****** [MCCUTCHEON \& JENNINGS, 1982]. INCLUDES TEMP COR-

C ***** RECTION, AS FORMULATED. MODIFIED HERE TO 20 DEG C AS AMBIENT

C ****** TEMPERATURE CORRECTIONS ARE PERFORMED IN MAIN.

20 IF (DEP.GT.2.26) GO TO 40

$\mathrm{XM}=2.279+.721 * \mathrm{DEP}$

$30 \mathrm{XK} 2=-1440 . * \mathrm{ALOG}(1 .-.00570 * \mathrm{SQRT}(\mathrm{XM}) / \mathrm{DEP}) / \mathrm{XM}$

RETURN

$40 \mathrm{XM}=13.94 *$ ALOG (DEP) -7.45

GO TO 30

$\mathrm{C} * * * * * *$

C ****** [LANGBEIN \& DURHAM, 1967]--FROM REGR. ANALYSIS OF SYNTH. DATA

$\mathrm{C} * * * * * *$

$50 \mathrm{XK} 2=7.61 * \mathrm{U} /(\mathrm{DEP} * * 1.33)$

RETURN

C $* * * * * * *$

C ***** [PADDEN \& GLOYNA, [1971]

C $* * * * * * *$

$60 \mathrm{XK} 2=6.86 *(U * * .703) /(\mathrm{DEP} * * 1.054)$

RETURN

$\mathrm{C} * * * * * *$

C ****** [BANSAL, 1973]

C $* * * * * * *$

$70 \mathrm{XK} 2=4.66 *(\mathrm{U} * * .6) /(\mathrm{DEP} * * 1.4)$

RETURN

C ********

C ***** [PARKHURST \& POMEROY, 1972]

C ********

$80 \mathrm{XK} 2=48.4 *(1 .+.17 * U * * 2 / \mathrm{DEP} * 32.2) *(U * \mathrm{~S}) * * .375 / \mathrm{DEP}$ RETURN

C $* * * * x *$

REAER.

REAER.

REAER.

REAER .

REAER.

REAER .

REAER .

REAER.

REAER.

REAER .

REAER.

REAER.

REAER .

REAER.

REAER.

REAER .

REAER .

REAER.

REAER .

REAER.

REAER .

REAER.

REAER.

REAER.

REAER.

REAER .

REAER.

REAER .

REAER .

REAER .

REAER.

REAER.

REAER.

REAER.

REAER.

REAER .

REAER.

REAER.

REAER.

REAER .

REAER.

REAER.

REAER.

REAER.

REAER .

REAER.

REAER .

REAER .

REAER. 
APPENDIX I--LISTING OF COMPUTER CODE--Continued.

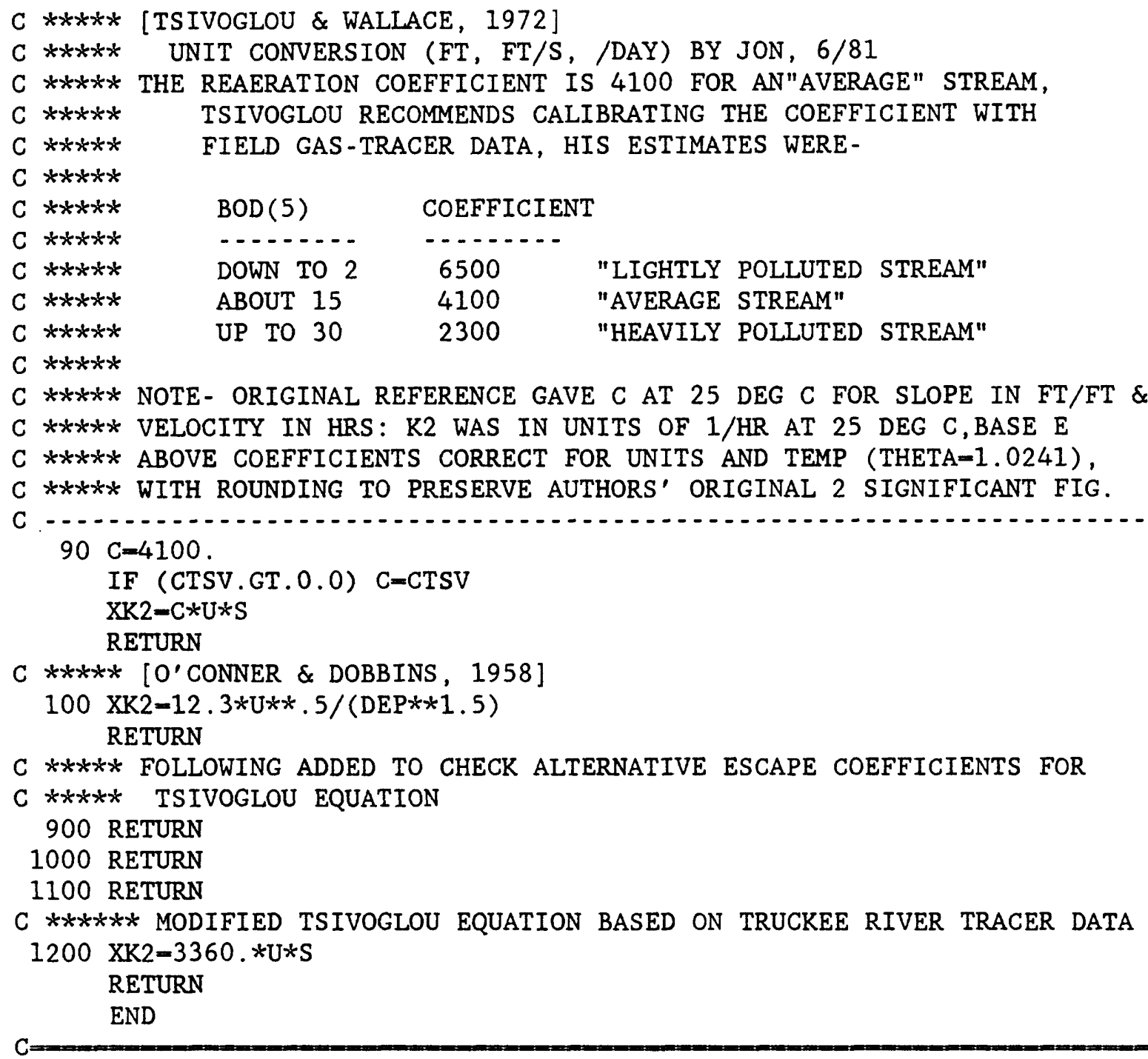

SUBROUTINE PPLOT (NPLOT, PTITLE, YTITLE, YN, YMAX, YMIN)

C

C ****** SUBROUTINE TO SET UP LINEPRINTER OUTPUT

C $* * * * * *$ PROGRAMMED BY JON $1 / 82$

C

C $* * * * *$ VARIABLES COMMON TO ONE OR MORE OF MAIN, READCD, ROUTE, NITRIF,

C $* * * * * *$

C ****** SCALARS:

C $* x * * * x$

COMMON /VALL/ BDN, BNT, CARD, DELNH4, DELNO2, DELTT, DXPNT, EA,

1 ER, ICDBUF, IEND, IGRAPH, ITYPE, ILIN, IMODEL, INITSW, IPLOT,

2 IPO4, IPRMIN, IPNET, IRET, ISW1, ISWBAD, ITAB1, ITAB2, ITEST,

3 ITFLAG, IXGEOM, J, JJ, JSEG, KPLOT, K2T, KCT, KCRT, KORNRT,

4 KORNFT, KNH4RT, KNH4FT, KNO2RT, KNO2FT, KNO3RT, KPO4BT, KNCR1T,

5 KNCR2T, LNCNTR, MPT, NCONSV, NNCONS, NSEG, PRINT, QTOT, TT,

6 TTSUM, XEND, XL, XSTRT, IRESP, DATE
REAER .

REAER.

REAER.

REAER.

REAER.

REAER.

REAER.

REAER.

REAER.

REAER.

REAER.

REAER.

REAER.

REAER.

REAER.

REAER.

REAER.

REAER .

REAER.

REAER .

REAER .

REAER .

REAER.

REAER.

REAER .

REAER.

REAER.

REAER .

REAER .

REAER.

REAER .

REAER .

REAER .

PPLOT.

PPLOT.

PPLOT.

PPLOT.

PPIOT.

COMMON

COMMON

COMMON

COMMON

COMMON

COMMON

COMMON

COMMON

COMMON

COMMON

COMMON

COMMON 
APPENDIX I--LISTING OF COMPUTER CODE--Continued.

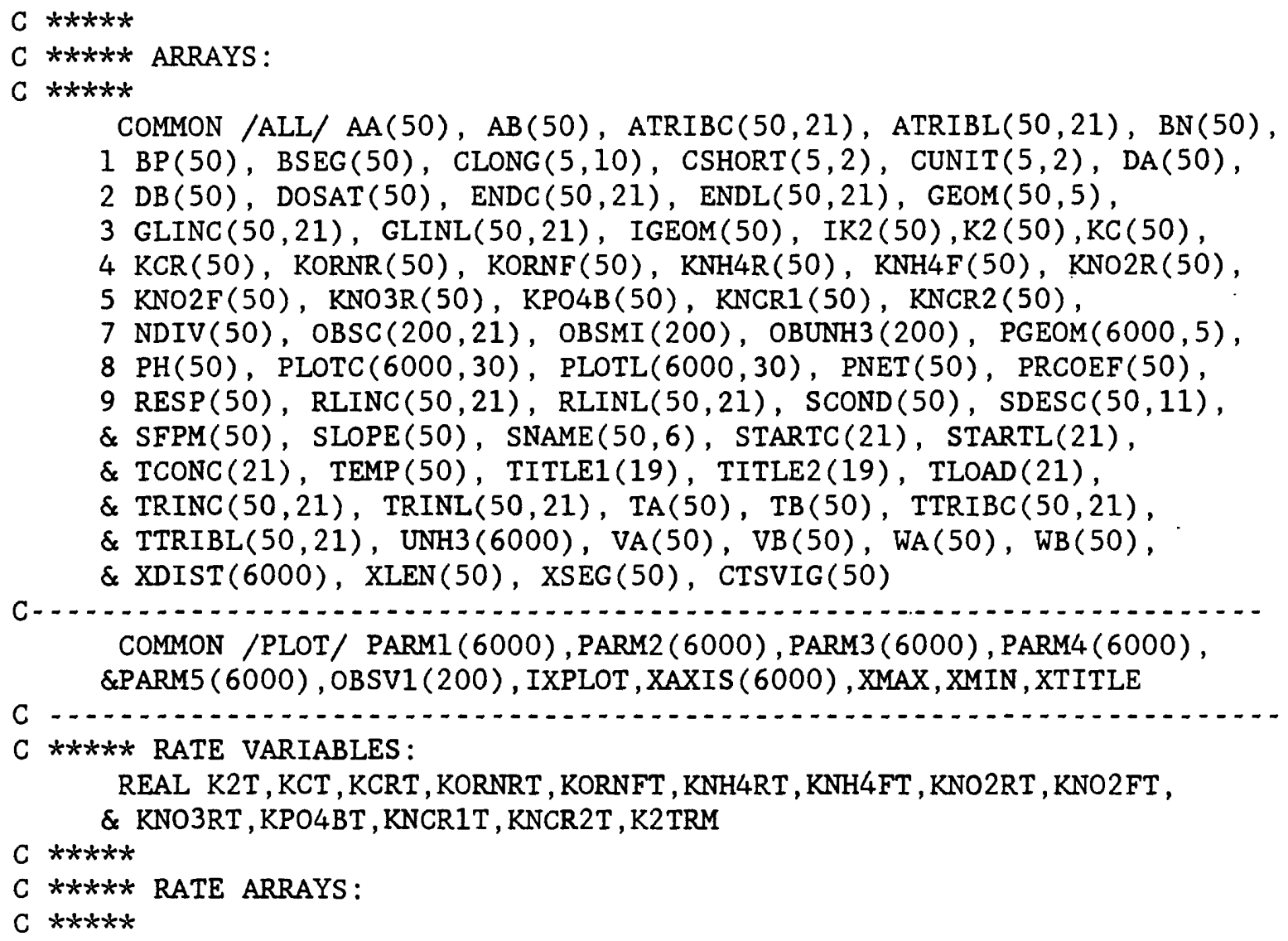

REAL K2T , KCT , KCRT, KORNRT , KORNFT , KNH4RT, KNH4FT, KNO2RT , KNO2FT,

COMMON

COMMON

COMMON

COMMON

COMMON

COMMON

COMMON

COMMON

COMMON

COMMON

COMMON

COMMON

COMMON

COMMON

COMMON

COMMON

COMMON

COMMON

COMMON

COMMON

COMMON

PPLOT.

PPLOT.

PPLOT.

PPLOT.

PPLOT.

PPLOT.

PPLOT.

PPLOT.

PPLOT.

PPLOT.

PPLOT.

PPLOT.

PPLOT.

PPLOT.

PPLOT.

PPLOT.

PPLOT.

PPLOT.

PPLOT.

PPLOT.

PPLOT.

PPLOT.

PPLOT.

PPLOT.

PPLOT.

PPLOT.

PPLOT.

PPLOT.

PPLOT. 
APPENDIX I--LISTING OF COMPUTER CODE--Continued.

$\begin{array}{ll}\text { C } * * * * * * 6 & \text { DO DEFICITS: CBOD, NO2, NH4 } \\ \text { C ******7 7 } & \text { DO DEFICITS: BENTHIC, R, P, K2 }\end{array}$

PPLOT.

C $* x * * * * x$ 4

PPLOT.

IF(KPLOT.EQ. 3) GOTO 45

IF (NPLOT.LE. 3) NPARM=1

IF (NPLOT.EQ.4) NPARM=3

IF (NPLOT.EQ.5) NPARM $=2$

IF (NPLOT.EQ.6) NPARM $=3$

IF (NPLOT.EQ.7) NPARM $=4$

CALL PMM(AMIN, AMAX, PARMI, KK)

IF (NPARM. EQ. 1) GOTO 20

CALL PMM(BMIN, BMAX, PARM2, KK)

AMAX-AMAX1 (AMAX, BMAX)

AMIN-AMIN1 (AMIN, BMIN)

IF (NPARM. EQ. 2) GOTO 20

CALL PMM(BMIN, BMAX, PARM3, KK)

AMAX-AMAXI (AMAX, BMAX)

AMIN=AMIN1 (AMIN, BMIN)

IF (NPARM. EQ . 3) GOTO 20

CALL PMM(BMIN, BMAX, PARM4, KK)

AMAX-AMAXI (AMAX, BMAX)

AMIN-AMIN1 (AMIN , BMIN)

IF (NPARM. EQ. 4) GOTO 20

CALL PMM(BMIN, BMAX, PARM5, KK)

AMAX-AMAXI (AMAX, BMAX)

AMIN=AMIN1 (AMIN, BMIN)

20 IF ((NPLOT.EQ.1).OR. (NPLOT.GE.5)) GOTO 25

CALL PMM(BMIN, BMAX, OBSVI, MPT)

IF ( (BMAX.EQ.0.).AND. (BMIN.EQ.0.)) NOBS $=0$

IF (BMAX. EQ. BMIN) GOTO 25

AMAX-AMAXI (AMAX, BMAX)

AMIN=AMIN1 (AMIN, BMIN)

C ******* OPTIONAL 'FLOATING' MAX AND MINS FOR PLOTS

25 IF(KPLOT.NE.1) GOTO 27

IF(AMAX.GT.0.1) GOTO 26

YMAX $=.10$

YMIN $=0.0$

IF (AMIN.LT. (-.1)) YMIN=AMIN-.1

IF ((AMIN.LT . $(-.01))$. AND . (AMIN.GT . $(-.1))$ ) YMIN $=-.1$ GOTO 45

26 CONTINUE

TMIN=FLOAT (IFIX (AMIN))

IF (TMIN. LT . - 1.) AMIN=AMIN -1.0

$D I F F=A M I N-T M I N$

IF ((DIF.EQ. (0.0)) .AND. (TMIN.GT.1.)) TMIN=TMIN-1.0

YMIN=TMIN

TMAX-FLOAT (IFIX (AMAX))

IF (TMAX.GE.1.) YMAX=TMAX+1.0

IF (TMAX.LT. 1.) YMAX $=1$.

GOTO 45

PPLOT.

PPLOT.

PPLOT.

PPLOT.

PPLOT.

PPLOT.

PPLOT.

PPLOT.

PPLOT.

PPLOT.

PPLOT.

PPLOT.

PPLOT.

PPLOT.

PPLOT.

PPLOT.

PPLOT.

PPLOT.

PPLOT.

PPLOT.

PPLOT.

PPLOT.

PPLOT.

PPLOT.

PPLOT.

PPLOT.

PPLOT.

PPLOT.

PPLOT .

PPLOT.

PPLOT.

PPLOT.

PPLOT.

PPLOT.

PPLOT.

PPLOT.

PPLOT.

PPLOT.

PPLOT.

PPLOT.

PPLOT.

PPLOT.

PPLOT.

PPLOT.

PPLOT.

PPLOT.

PPLOT.

PPLOT. 
APPENDIX I--LISTING OF COMPUTER CODE--Continued.

C $* * * * * *$ FOR Y'S $>0$., SET MAX AND MIN VALUES TO MULTIPLES OF 1 OR 5

27 IF (AMAX.LE.0.) YMAX $=0$.

IF (YMAX.EQ.0) GOTO 45

EMAX-FLOAT (IFIX (ALOG10(AMAX)))

TMAX-AMAX/10.**EMAX

TMIN=FLOAT $($ IFIX $($ AMIN $/(10 * * \operatorname{EMAX})))$

IF(TMIN.LT.0.) AFIX $=-1$.

IF (TMIN.LT.0.) TMIN=TMIN-1.0

TDELT-TMAX - TMIN

IF(TDELT.LE. 20.) TMAX-TMIN+20.

IF (TDELT.LE. 10.) TMAX-TMIN+10.

IF (TDELT.LE. 5.) TMAX=TMIN+5.

IF (TDELT.LE.2.5) TMAX-TMIN+2.5

IF (TDELT.LE. 2.0) TMAX-TMIN+2.0

IF (TDELT.LE. 1.) TMAX $=$ TMIN+1.

YMAX-TMAX* $(10 * *$ EMAX $)$

YMIN-TMIN* $(10 * *$ EMAX)

45 WRITE(PRINT, 100) TITLE2, PTITLE

48 CALL PLOT2 (GRAPH, XMAX, XMIN, YMAX, YMIN, PRINT)

GOTO $(50,50,55,60,65,70,75)$, NPLOT

C $* * * * *$ PLOT 1 PARAMETER

50 CALL PLOT3 ('*', XAXIS, PARM1, KK, GRAPH)

IF ( (NPLOT.EQ.2) . AND . (NOBS.EQ.1))

\& CALL PLOT3 (' $X$ ' , OBSMI, OBSV1, MPT, GRAPH)

CALL PLOT4 (YN, YTITLE, GRAPH)

IF(NPLOT.EQ.1) WRITE(PRINT, 110) XTITLE

IF (NPLOT.EQ. 2) WRITE (PRINT, 115) XTITLE

GOTO 80

55 CONTINUE

C ****** PLOT DO SATURATION

56 CALL PLOT2 (GRAPH, XMAX, XMIN, YMAX, YMIN, PRINT)

CALL PLOT3 (' +' , XAXIS , PARM1, KK, GRAPH)

CALL PLOT3 (' $*$ ' , XAXIS, PARM2, KK, GRAPH)

IF (NOBS.EQ. 1) CALL PLOT3 (' $X$ ' , OBSMI, OBSVI, MPT, GRAPH)

CALI PLOT4 (YN, YTITLE, GRAPH)

WRITE(PRINT, 120)XTITLE

GOTO 80

60 CONTINUE

C $* x * * * *$ PLOT DO

IF(KPLOT.EQ.0) GOTO 62

GOTO 63

62 IF(AMAX.LE. 10.) YMAX $=10$.

IF ( (AMAX.GT. 10.) . AND. (AMAX.LE. 20.)) $Y M A X=20$.

IF (AMAX.GT.20.) YMAX=AMAX

YMIN $=0$.

63 CALL PLOT2 (GRAPH, XMAX, XMIN, YMAX, YMIN, PRINT)

CALL PLOT3 (' +' , XAXIS , PARM1, KK, GRAPH)

CALL PLOT3 (' *', XAXIS , PARM2, KK, GRAPH)

IF (NOBS . EQ . 1) CALL PLOT3 (' $X$ ' , OBSMI , OBSV1, MPT, GRAPH)

CALL PLOT4(YN, YTITLE, GRAPH)

PPLOT.

PPLOT.

PPLOT.

PPLOT.

PPLOT.

PPLOT.

PPLOT.

PPLOT.

PPLOT.

PPLOT.

PPLOT.

PPLOT.

PPLOT.

PPLOT.

PPLOT.

PPLOT.

PPLOT.

PPLOT.

PPLOT.

PPLOT.

PPLOT.

PPLOT.

PPLOT.

PPLOT.

PPLOT.

PPLOT.

PPLOT.

PPLOT.

PPLOT.

PPLOT.

PPLOT.

PPLOT.

PPLOT.

PPLOT.

PPLOT.

PPLOT.

PPLOT.

PPLOT.

PPLOT.

PPLOT.

PPLOT.

PPLOT.

PPLOT.

PPLOT.

PPLOT.

PPLOT.

PPLOT.

PPLOT.

PPLOT.

PPLOT. 
APPENDIX I--LISTING OF COMPUTER CODE--Continued.

WRITE(PRINT, 125) XTITLE

GOTO 80

C ****** PLOT CBOD DEFICITS

65 CALL PLOT3 (' $C$ ' , XAXIS , PARM1, KK, GRAPH)

CALL PLOT3 (' $N$ ', XAXIS, PARM2, KK, GRAPH)

CALL PLOT4 (YN, YTITLE, GRAPH)

WRITE(PRINT, 130) XTITLE

GOTO 80

C $* * * * *$ PLOT N-CYCLE DEFICITS

70 CALL PLOT3 (' $\mathrm{C}$ ', XAXIS, PARMI, KK, GRAPH)

CALL PLOT3 (' $\mathrm{N}$ ', XAXIS, PARM2, KK, GRAPH)

CALL PLOT3 ('A', XAXIS, PARM3, KK, GRAPH)

CALL PLOT4 (YN, YTITLE, GRAPH)

WRITE (PRINT, 135) XTITLE

GOTO 80

C $* * * * *$ PLOT $\mathrm{P} \& \mathrm{R}$ DEFICITS

75 CALL PLOT3 ('B', XAXIS, PARM1, KK, GRAPH)

CALL PLOT3 ('R', XAXIS , PARM2, KK, GRAPH)

CALL PLOT3 (' $P$ ' , XAXIS , PARM3, KK, GRAPH)

CALL PLOT3 (' $\mathrm{K}$ ', XAXIS , PARM4, KK, GRAPH)

CALL PLOT4 (YN, YTITLE, GRAPH)

WRITE (PRINT, 140) XTITLE

80 DO 85 I $=1,2000$

PARM1 (I) $=0$.

$\operatorname{PARM} 2(I)=0$.

$\operatorname{PARM} 3(I)=0$.

$\operatorname{PARM} 4(I)=0$.

85 PARM5 (I) $=0$.

DO $90 \quad I=1,200$

$90 \operatorname{OBSV1}(\mathrm{I})=0$. RETURN

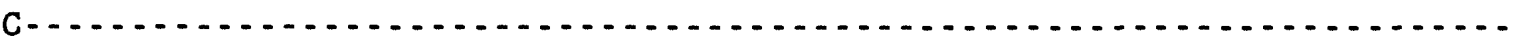

100 FORMAT ( $1 \mathrm{H} 1,16 \mathrm{X}$, 'U.S. GEOLOGICAL SURVEY, WATER RESOURCES DIVISION'

$\&, ':^{\prime}, 2 \mathrm{X}$, 'TRUCKEE RIVER WATER-QUALITY MODEL', 15X,' [REV 87.9]',

$\& / 1 \mathrm{X}, 130\left(^{\prime}\right.$ - $\left.\left.^{\prime}\right), / 4 \mathrm{X}, / 20 \mathrm{X}, 19 \mathrm{~A} 4 / 20 \mathrm{X}, 15 \mathrm{~A} 4\right)$

110 FORMAT $\left(/ 51 \mathrm{X}, 11 \mathrm{~A} 1, / / 10 \mathrm{X},{ }^{\prime}\right.$ EXPLANATION: * - CALCULATED VALUES.' $)$

115 FORMAT $(/ 51 \mathrm{X}, 11 \mathrm{~A} 1, / / 10 \mathrm{X}$, 'EXPLANATION: * = CALCULATED VALUES, ', $\&^{\prime} \mathrm{X}=$ OBSERVED.')

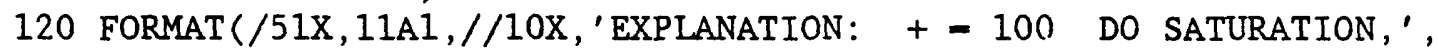
$\&^{\prime} *$ - CALCULATED DO SATURATION, X - OBSERVED. ')

125 FORMAT $(/ 51 \mathrm{X}, 11 \mathrm{Al}, / / 10 \mathrm{X}$, ' EXPLANATION: + - DO AT 100 SATURATION, ', $\&^{\prime} \mathrm{D}=$ CALCULATED DO DEFICIT,$*=$ CALCULATED DO, $\mathrm{X}=$ OBSERVED. ${ }^{\prime}$ )

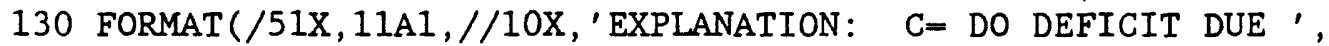
\&'TO CBOD')

1.35 FORMAT $(/ 51 \mathrm{X}, 11 \mathrm{Al}, / / 10 \mathrm{X}$, ' EXPLANATION: $\mathrm{C}=$ DO DEFICIT DUE TO ', $\&^{\prime}$ CBOD, A - DEFICIT DUE TO NH4 OXIDATION, $\mathrm{N}=$ DEFICIT DUE TO NO2 ', \&'OXIDATION', /24X, 'D = TOTAL DEFICIT')

140 FORMAT (/51X,11A1,//10X,' EXPLANATION: B = DO DEFICIT DUE TO ', \&'BENTHIC DEPOSITS, $R$ = DEFICIT DUE TO RESPIRATION,' , $\& / 24 \mathrm{X}, ' \mathrm{P}-$ DEFICIT DUE TO PHOTOSYNTHESIS, $\mathrm{K}=$ DEFICIT DUE TO ',

PPLOT.

PPLOT.

PPLOT.

PPLOT.

PPLOT.

PPLOT.

PPLOT.

PPLOT.

PPLOT.

PPLOT.

PPLOT.

PPLOT.

PPLOT.

PPLOT.

PPLOT.

PPLOT.

PPLOT.

PPLOT.

PPLOT.

PPLOT.

PPLOT.

PPLOT.

PPLOT.

PPLOT.

PPLOT.

PPLOT.

PPLOT.

PPLOT.

PPLOT.

PPLOT.

PPLOT.

PPLOT.

MAIN. .

MAIN. .

MAIN. .

PPLOT.

PPLOT.

PPLOT.

PPLOT.

PPLOT.

PPLOT.

PPLOT.

PPLOT.

PPLOT.

PPLOT.

PPLOT.

PPLOT.

PPLOT.

PPLOT.

PPLOT. 
APPENDIX I--LISTING OF COMPUTER CODE--Continued.

\&'REAERATION' $, / 24 \mathrm{X},{ }^{\prime} \mathrm{D}=$ TOTAL DEFICIT' $\quad$ PPLOT. END

SUBROUTINE PRPLOT

C 16 MARCH 73

C GENERAL LINE-PRINTER PLOTTER OF UNKNOWN HISTORIC ORIGIN

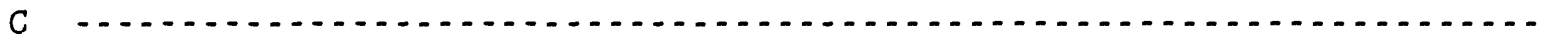

LOGICAL*1 WL, KPLT, KPLT1, KPLT2, KBOTGL, KORD , KABSC , KNHOR

DIMENSION NSCALE (5), ABNOS (26), X(1),Y(1)

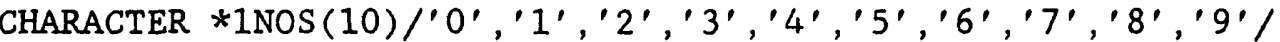

LOGICAL *1 IMAGE (1), CH, LABEL(1), BLI

LOGICAL *1 ERR1, ERR3, ERR5

LOGICAL *1 VC, HC, NC

CHARACTER *1 FOR1(19), FOR2(15), FOR3(19), BL, HF , HF1

CHARACTER $* 8$ FOX1(3), FOX2(2), FOX3(3)

EQUIVALENCE (FOR1, FOX1), (FOR2, FOX2), (FOR3, FOX3)

INTEGER PRINT

$C * * * * * *$

DATA HC/' -'/, NC/'+'/,BL/' '/, HF/'F'/, HFl/'.'/, VC/'/'/,BLl/' '/

DATA FOX1/' (1XA1,F9','.2, 121','A1) '/

DATA FOX2/' (1XA1,9', 'X121A1) '/

DATA FOX3/'(1H F .',', F ',', ) '/

DATA KPLT1/.FALSE./,KPLT2/.FALSE./

DATA KABSC, KORD, KBOTGL/3*.FALSE./

$C * * * * * *$

$10 \mathrm{KPLT}=$. TRUE.

IF (NV.LE. 25) GO TO 20

KPLT $=$. FALSE.

ERR3=. TRUE .

RETURN

20 CONTINUE

NVM-NV - 1

$\mathrm{NVP}-\mathrm{NV}+1$

$\mathrm{NDH}=\mathrm{NH} * \mathrm{NSH}$

$\mathrm{NDHP}=\mathrm{NDH}+1$

$\mathrm{NDV}=\mathrm{NV} * \mathrm{NSV}$

$\mathrm{NDVP}=\mathrm{NDV}+1$

NIMG $=($ NDHP $*$ NDVP $)$

IF (NDV.LE.120) GO TO 30

KPLT=. FALSE .

ERR5= . TRUE .

RETURN

30 CONTINUE

IF (NSCL.EQ.0) GO TO 40

FSY $=10 . * *$ NSCALE $(2)$

FSX $-10 . * *$ NSCALE $(4)$

IY-MINO (IABS (NSCALE (3)) , 7) +1

IX $=\operatorname{MINO}(\operatorname{IABS}(\operatorname{NSCALE}(5)), 9)+1$

GO TO 50

40 FSY $=1$.

PPLOT.

PRPLOT

PRPLOT

PRPLOT

PRPLOT

PRPLOT

PRPLOT

PRPLOT

PRPLOT

PRPLOT

PRPLOT

PRPLOT

PRPLOT

PRPLOT

PRPLOT

PRPLOT

PRPLOT

PRPLOT

PRPLOT

PRPLOT

PRPLOT

PRPLOT

PRPLOT

PRPLOT

PRPLOT

PRPLOT

PRPLOT

PRPLOT

PRPLOT

PRPLOT

PRPLOT

PRPLOT

PRPLOT

PRPLOT

PRPLOT

PRPLOT

PRPLOT

PRPLOT

PRPLOT

PRPLOT

PRPLOT

PRPLOT

PRPLOT

PRPLOT

PRPLOT

PRPLOT

PRPLOT

PRPLOT

PRPLOT 
APPENDIX I--LISTING OF COMPUTER CODE--Continued.

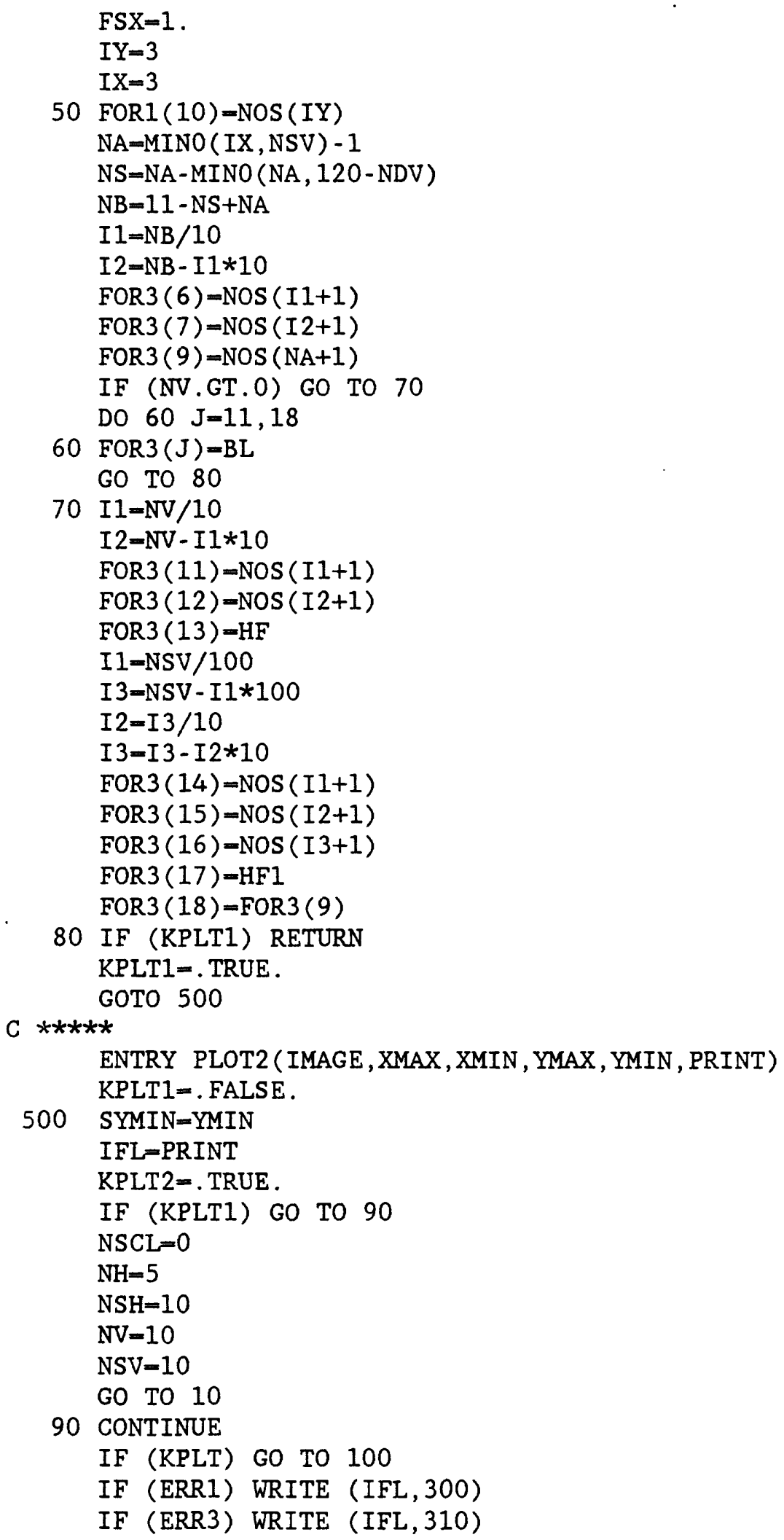

PRPLOT

PRPLOT

PRPLOT

PRPLOT

PRPLOT

PRPLOT

PRPLOT

PRPLOT

PRPLOT

PRPLOT

PRPLOT

PRPLOT

PRPLOT

PRPLOT

PRPLOT

PRPLOT

PRPLOT

PRPLOT

PRPLOT

PRPLOT

PRPLOT

PRPLOT

PRPLOT

PRPLOT

PRPLOT

PRPLOT

PRPLOT

PRPLOT

PRPLOT

PRPLOT

PRPLOT

PRPLOT

PRPLOT

PRPLOT

PRPLOT

PRPLOT

PRPLOT

PRPLOT

PRPLOT

PRPLOT

PRPLOT

PRPLOT

PRPLOT

PRPLOT

PRPLOT

PRPLOT

PRPLOT

PRPLOT

PRPLOT

PRPLOT 
APPENDIX I--LISTING OF COMPUTER CODE--Continued.

IF (ERR5) WRITE (IFL, 320)

PRPLOT

RETURN

100 YMX-YMAX

$\mathrm{DH}=(\mathrm{YMAX}-\mathrm{YMIN}) /$ FLOAT $(\mathrm{NDH})$

PRPLOT

$D V=(X M A X-X M I N) / F L O A T(N D V)$

PRPLOT

DO $110 \quad I=1$, NVP

PRPLOT

PRPLOT

PRPLOT

$110 \operatorname{ABNOS}(I)=(\mathrm{XMIN}+\mathrm{FLOAT}((\mathrm{I}-1) * \mathrm{NSV}) * \mathrm{DV}) * \mathrm{FSX}$

PRPLOT

DO $120 \quad I=1$, NIMG

PRPLOT

$120 \operatorname{IMAGE}(\mathrm{I})=\mathrm{BLI}$

DO $160 \quad I=1$, NDHP

I2 $=I * N D V P$

$I 1=I 2-N D V$

$\mathrm{KNHOR}=\mathrm{MOD}(\mathrm{I}-1, \mathrm{NSH}) . \mathrm{NE}, 0$

IF (KNHOR) GO TO 140

DO $130 \mathrm{~J}=\mathrm{I} 1, \mathrm{I} 2$

$130 \operatorname{IMAGE}(\mathrm{J})=\mathrm{HC}$

140 CONTINUE

DO $160 \mathrm{~J}=\mathrm{I} 1, \mathrm{I} 2$, NSV

IF (KNHOR) GO TO 150

$\operatorname{IMAGE}(\mathrm{J})=\mathrm{NC}$

GO TO 160

$150 \operatorname{IMAGE}(\mathrm{J})=\mathrm{VC}$

160 CONTINUE

$X M I N 1=X M I N-D V / 2$.

YMIN1 $=$ YMIN - DH $/ 2$.

RETURN

C $* * x * * * x$

ENTRY PLOT3 ( $\mathrm{CH}, \mathrm{X}, \mathrm{Y}, \mathrm{N} 3$, IMAGE)

IF (KPLT2) GO TO 180

170 WRITE (IFL, 330)

180 CONTINUE

IF (.NOT.KPLT) RETURN

IF (N3.GT.O) GO TO 190

KPLT $=$. FALSE .

WRITE (IFL, 340)

RETURN

190 DO $260 \mathrm{I}=1, \mathrm{~N} 3$

IF (DV) $210,200,210$

200 DUM $1=0$

GO TO 220

210 CONTINUE

DUMI $=(\mathrm{X}(\mathrm{I})-\mathrm{XMIN1}) / \mathrm{DV}$

220 IF (DH) $240,230,240$

230 DUM $2=0$

GO TO 250

240 CONTINUE

DUM2 $=(Y(I)-Y M I N 1) / D H$

250 CONTINUE

IF (DUM1.LT.0..OR.DUM2.LT.0.) GO TO 260

IF (DUM1.GE.NDVP.OR.DUM2.GE.NDHP) GO TO 260

PRPLOT

PRPLOT

PRPLOT

PRPLOT

PRPLOT

PRPLOT

PRPLOT

PRPLOT

PRPLOT

PRPLOT

PRPLOT

PRPLOT

PRPLOT

PRPLOT

PRPLOT

PRPLOT

PRPLOT

PRPLOT

PRPLOT

PRPLOT

PRPLOT

PRPLOT

PRPLOT

PRPLOT

PRPLOT

PRPLOT

PRPLOT

PRPLOT

PRPLOT

PRPLOT

PRPLOT

PRPLOT

PRPLOT

PRPLOT

PRPLOT

PRPLOT

PRPLOT

PRPLOT

PRPLOT

PRPLOT

PRPLOT

PRPLOT 
APPENDIX I--LISTING OF COMPUTER CODE--Continued.

\begin{tabular}{|c|c|c|}
\hline & $\mathrm{NX}=1+\operatorname{INT}(\mathrm{DUM} 1)$ & PRPLOT \\
\hline & $\mathrm{NY}=1+\mathrm{INT}(\mathrm{DUM} 2)$ & PRPLOT \\
\hline & $\mathrm{J}=(\mathrm{NDHP}-\mathrm{NY}) * \mathrm{NDVP}+\mathrm{NX}$ & PRPLOT \\
\hline & $\operatorname{IMAGE}(\mathrm{J})=\mathrm{CH}$ & PRPLOT \\
\hline 260 & CONTINUE & PRPLOT \\
\hline & RETURN & PRPLOT \\
\hline$C * * *$ & & PRPLOT \\
\hline & ENTRY PLOT4 (NL, LABEL, IMAGE) & PRPLOT \\
\hline & IF (.NOT.KPLT) RETURN & PRPLOT \\
\hline & IF (.NOT.KPLT2) GO TO 170 & PRPLOT \\
\hline & DO $280 I=1, \mathrm{NDHP}$ & PRPLOT \\
\hline & IF (I.EQ.NDHP.AND.KBOTGL) GO TO 280 & PRPLOT \\
\hline & $\mathrm{WL}=\mathrm{BL1}$ & PRPLOT \\
\hline & IF (I.LE.NL) WL=IABEL(I) & PRPLOT \\
\hline & $I 2=I * N D V P$ & PRPLOT \\
\hline & $I 1=I 2-N D V$ & PRPLOT \\
\hline & IF (MOD (I-1,NSH).EQ.0.AND..NOT.KORD) GO TO 270 & PRPLOT \\
\hline & WRITE (IFL, FOR2) WL, (IMAGE (J), J=I1,I2) & PRPLOT \\
\hline & GO TO 280 & PRPLOT \\
\hline 270 & CONTINUE & PRPLOT \\
\hline & ORDNO $=(Y M X-F L O A T(I-1) * D H) * F S Y$ & PRPLOT \\
\hline & IF (I.EQ.NDHP) ORDNO=SYMIN & PRPLOT \\
\hline & WRITE (IFL, FOR1) WL, ORDNO, (IMAGE (J), J=I1, I2) & PRPLOT \\
\hline 280 & CONTINUE & PRPLOT \\
\hline & IF (KABSC) GO TO 290 & PRPLOT \\
\hline & WRITE (IFL, FOR3) (ABNOS (J), J=1, NVP) & PRPLOT \\
\hline 290 & CONTINUE & PRPLOT \\
\hline & DO $292 I=1,2000$ & PRPLOT \\
\hline 292 & $\operatorname{IMAGE}(I)=B L 1$ & PRPLOT \\
\hline & RETURN & PRPLOT \\
\hline$C * * * x=$ & & PRPLOT \\
\hline$C * * * 3$ & & PRPLOT \\
\hline$C * * * 3$ & & PRPLOT \\
\hline 300 & FORMAT (T5,'SOME PLOT1 ARG. ILLEGALLY $0^{\prime}$ ) & PRPLOT \\
\hline 310 & FORMAT (T5, NO. OF VERTICAL LINES $>25^{\prime}$ ) & PRPLOT \\
\hline 320 & FORMAT (T5,'WIDTH OF GRAPH $>121$ ') & PRPLOT \\
\hline 330 & FORMAT (T5,' PLOT2 MUST BE CALLED') & PRPLOT \\
\hline 340 & FORMAT (T5,'PLOT3,ARG2 ) $\left.0^{\prime}\right)$ & PRPLOT \\
\hline & END & PRPLOT \\
\hline$C=$ & SUBROUTINE PMM $(X M N, X M X, A, N)$ & $\begin{array}{l}\text { PMM. . } \\
\text { PMM. . }\end{array}$ \\
\hline C.... & $\begin{array}{l}n_{1} \\
\end{array}$ & PMM. . . \\
\hline & DIMENSION A(1) & PMM. \\
\hline & IF (N.LT.2) GO TO 20 & PMM. . . \\
\hline & $\mathrm{XMN}=\mathrm{A}(1)$ & PMM. \\
\hline & $\mathrm{XMX}=\mathrm{XMN}$ & PMM. . \\
\hline & DO $10 I=2, N$ & PMM. . . \\
\hline & IF $(A(I) . L T . X M N) X M N=A(I)$ & PMM. . . \\
\hline & IF $(A(I) . G T \cdot X M X) \quad X M X=A(I)$ & PMM. . \\
\hline 10 & CONTINUE & PMM. . . \\
\hline
\end{tabular}


APPENDIX I--LISTING OF COMPUTER CODE--Continued.

$\begin{array}{ll} & \text { RETURN } \\ & \text { IF (N.LT.1) GO TO } 30 \\ & \text { XMN }=A(1) \\ & \text { XMX-XMN } \\ & \text { RETURN } \\ 30 & \text { XMN=0.0 } \\ & \text { XMX }=0.0 \\ & \text { RETURN } \\ & \text { END }\end{array}$

C

$\mathrm{C}$

C

C $* * * x *$

C $* * * * *$ SCALARS:

$\mathrm{C} * * * * *$

COMMON /VALL/ BDN, BNT, CARD, DELNH4, DELNO2, DELTT, DXPNT, EA,

1 ER, ICDBUF, IEND, IGRAPH, ITYPE, ILIN, IMODEL, INITSW, IPLOT,

2 IP04, IPRMIN, IPNET, IRET, ISW1, ISWBAD, ITAB1, ITAB2, ITEST,

3 ITFLAG, IXGEOM, J, JJ, JSEG, KPLOT, K2T, KCT, KCRT, KORNRT,

4 KORNFT, KNH4RT, KNH4FT, KNO2RT, KNO2FT, KNO3RT, KP04BT, KNCR1T,

5 KNCR2T, LNCNTR, MPT, NCONSV, NNCONS, NSEG, PRINT, QTOT, TT,

6 TTSUM, XEND, XL, XSTRT, IRESP, DATE

C $* * * * * *$

C $* * * * *$ ARRAYS:

$C * * * * * *$

COMMON /ALL/ $\mathrm{AA}(50), \operatorname{AB}(50), \operatorname{ATRIBC}(50,21), \operatorname{ATRIBL}(50,21), \operatorname{BN}(50)$,

$1 \mathrm{BP}(50), \operatorname{BSEG}(50), \operatorname{CLONG}(5,10), \operatorname{CSHORT}(5,2), \operatorname{CUNIT}(5,2), \operatorname{DA}(50)$,

$2 \mathrm{DB}(50), \operatorname{DOSAT}(50), \operatorname{ENDC}(50,21), \operatorname{ENDL}(50,21), \operatorname{GEOM}(50,5)$,

$3 \operatorname{GLINC}(50,21), \operatorname{GLINL}(50,21), \operatorname{IGEOM}(50), \operatorname{IK} 2(50), \operatorname{K} 2(50), \mathrm{KC}(50)$,

$4 \mathrm{KCR}(50), \operatorname{KORNR}(50), \operatorname{KORNF}(50), \operatorname{KNH} 4 \mathrm{R}(50), \operatorname{KNH} 4 \mathrm{~F}(50), \operatorname{KNO} 2 R(50)$,

$5 \mathrm{KNO} 2 \mathrm{~F}(50), \mathrm{KNO} 3 \mathrm{R}(50), \mathrm{KPO4B}(50), \mathrm{KNCR} 1(50), \mathrm{KNCR} 2(50)$,

$7 \operatorname{NDIV}(50), \operatorname{OBSC}(200,21), \operatorname{OBSMI}(200), \operatorname{OBUNH} 3(200), \operatorname{PGEOM}(6000,5)$,

$8 \mathrm{PH}(50), \operatorname{PLOTC}(6000,30), \operatorname{PLOTL}(6000,30), \operatorname{PNET}(50), \operatorname{PRCOEF}(50)$,

$9 \operatorname{RESP}(50), \operatorname{RLINC}(50,21), \operatorname{RLINL}(50,21), \operatorname{SCOND}(50), \operatorname{SDESC}(50,11)$,

$\& \operatorname{SFPM}(50), \operatorname{SLOPE}(50), \operatorname{SNAME}(50,6), \operatorname{STARTC}(21), \operatorname{STARTL}(21)$,

$\& \operatorname{TCONC}(21), \operatorname{TEMP}(50), \operatorname{TITLE} 1(19), \operatorname{TITLE} 2(19), \operatorname{TLOAD}(21)$,

$\& \operatorname{TRINC}(50,21), \operatorname{TRINL}(50,21), \operatorname{TA}(50), \operatorname{TB}(50), \operatorname{TTRIBC}(50,21)$,

$\& \operatorname{TTRIBL}(50,21), \operatorname{UNH} 3(6000), \mathrm{VA}(50), \mathrm{VB}(50), \mathrm{WA}(50), \mathrm{WB}(50)$,

$\& \operatorname{XDIST}(6000), \operatorname{XLEN}(50), \operatorname{XSEG}(50), \operatorname{CTSVIG}(50)$

COMMON /PLOT/ PARMI (6000), PARM2 $(6000), \operatorname{PARM3}(6000), \operatorname{PARM4}(6000)$ \&PARM5 (6000), OBSV1(200), IXPLOT, XAXIS (6000), XMAX, XMIN , XTITLE

C

C $* * * * *$ RATE VARIABLES :

REAL K2T , KCT , KCRT , KORNRT , KORNFT, KNH4RT, KNH4FT, KNO2RT , KNO2FT, $\mathrm{C} * * * * * *$
PMM. . .

PMM. . .

PMM . . .

PMM. . .

PMM. . .

PMM . . .

PMM. . .

PMM . . .

PMM. . .

READCD

READCD

READCD

READCD

COMMON

COMMON

COMMON

COMMON

COMMON

COMMON

COMMON

COMMON

COMMON

COMMON

COMMON

COMMON

COMMON

COMMON

COMMON

COMMON

COMMON

COMMON

COMMON

COMMON

COMMON

COMMON

COMMON

COMMON

COMMON

COMMON

COMMON

COMMON

COMMON

COMMON

COMMON

COMMON

COMMON

READCD

READCD

READCD

READCD 
APPENDIX I--LISTING OF COMPUTER CODE--Continued.

C ****** RATE ARRAYS:

READCD

$C * * * * * *$

REAL K2 , KCR , KC, KNR, KN, KORNR, KORNF , KNH4R, KNH4F, KNO2R, KNO2F, KNO3R, READCD \& KPO4B, KNCR1, KNCR2

READCD

READCD

INTEGER CARD, PRINT

READCD

CHARACTER $* 16$ DATE

READCD

INTEGER *2CHARST $(80)$, CARD1, CARD2, CARD3, CARD4, CARD5, CARD6

READCD

INTEGER *2CARD7, CARD8, CARD9, CARDA, CARDC

READCD

INTEGER *2CHERR

INTEGER *2BLANK

C $* * * * *$ SET-UP DATA FOR CARD TYPES:

DATA CARD1, CARD2, CARD3, CARD4, CARD5, CARD6, CARD7, CARD8 , CARD9, CARDA,

$1 \mathrm{CARDC} / 1 \mathrm{H} 1,1 \mathrm{H} 2,1 \mathrm{H} 3,1 \mathrm{H} 4,1 \mathrm{H} 5,1 \mathrm{H} 6,1 \mathrm{H} 7,1 \mathrm{H} 8,1 \mathrm{H} 9,1 \mathrm{HA}, 1 \mathrm{HC} /$

DATA BLANK/1H /

READCD

READCD

READCD

READCD

READCD

READCD

READCD

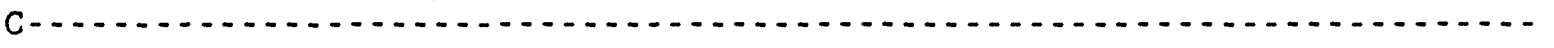

C INITIALIZE VAR.

ISECT $=1$

READCD

READCD

$\mathrm{MPT}=0$

READCD

$\mathrm{MPT} 1=0$

READCD

$\mathrm{KK}=0$

IEND=0

ISWBAD $=0$

I GOT $1=0$

ICONS $=1$

INCONS $=1$

ISECT $=1$

IRET $=0$

IDUM $=0$

$X D U M=0$.

READCD

READCD

READCD

READCD

READCD

READCD

READCD

READCD

READCD

READCD

C

C INITIALIZE REREAD CARD INPUT FUNCTION

READCD

READCD

READCD

C $* x+x * x$

C $* * * * *$ READ CARD IN BUFFER AND TEST FOR CHARACTERS NE NUMBERS, BLNK,

$C * * * * *$ PRINT CARD IMAGES IF ITEST NE 0, TEST FOR CARD NUMBERS,

C $* * * * *$ CHECK LEGIT CARDS FOR CHARACTER TYPES, BYPASS CARDS STARTING

C ****** WITH A 'C' AND BLANK CARDS

C $* * * * * * *$

30 READ (CARD, 40, END $=490$ ) CHARST

40 FORMAT (80A1)

IF(ITEST.NE.0) WRITE (PRINT, 41) CHARST

41 FORMAT ( $1 \mathrm{X}$, ' DATA-CARD IMAGE $=$ ', 80A1)

IF (CHARST (1).EQ. CARDC) GOTO 30

IF (CHARST (1).EQ.BLANK. OR.CHARST (2). EQ.BLANK) GOTO 30

READCD

READCD

READCD

READCD

READCD

READCD

READCD

READCD

READCD

READCD

READCD

READCD

BACKSPACE (5)

50 IF (CHARST (1). EQ. CARD1) GOTO 70

READCD

IF (CHARST (1) . EQ. CARDA) GOTO 60

IF (CHARST (1).EQ.CARD3) GO TO 390

READCD

READCD

READCD

CALL TEST (CHARST, IRET, CHERR)

READCD

READCD 
APPENDIX I--LISTING OF COMPUTER CODE--Continued.

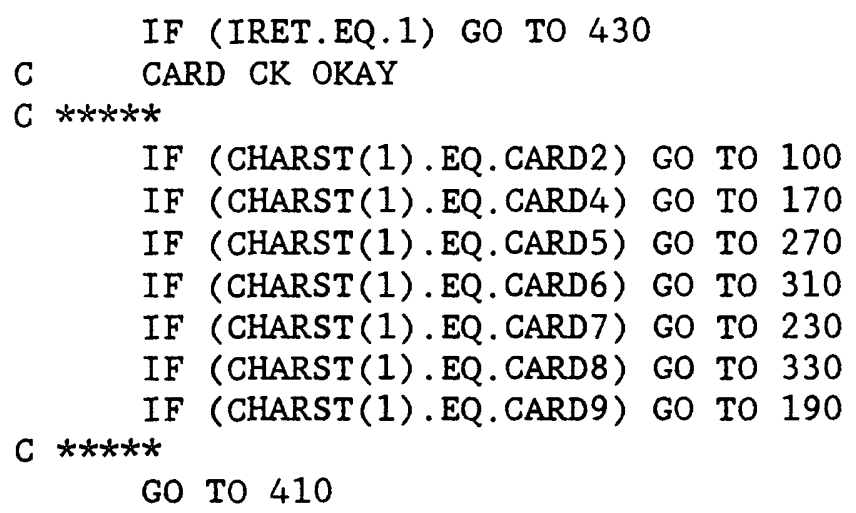

READCD

READCD

READCD

READCD

READCD

READCD

READCD

READCD

READCD

READCD

READCD

READCD

READCD

READCD

READCD

READCD

READCD

READCD

READCD

READCD

READCD

READCD

READCD

READCD

READCD

READCD

READCD

READCD

READCD

READCD

READCD

READCD

READCD

READCD

READCD

READCD

READCD

READCD

READCD

READCD

READCD

READCD

READCD

READCD

READCD

READCD

READCD

READCD

READCD

READCD 
APPENDIX I--LISTING OF COMPUTER CODE--Continued.

GO TO 30

READCD

170 READ (5, 180) K, IDUM, (GEOM(K,L), L $=1,5), \mathrm{VA}(\mathrm{K}), \mathrm{VB}(\mathrm{K}), \mathrm{TA}(\mathrm{K}), \mathrm{TB}(\mathrm{K})$
IAA(K), AB(K), DA(K), DB(K), WA(K), WB(K)
180 FORMAT (2X, I2, I1, I5(F5.0))
IF (K. GT.50) GO TO 450
GO TO 30

C $* * * * *$ "91" CARDS FOR READING IN OBSERVED DATA

190 CONTINUE

IF (CHARST(2).EQ.CARD2) GO TO 210

$\mathrm{MPT}=\mathrm{MPT}+1$

READ (5, 200) OBSMI (MPT), OBSC (MPT , 5), OBSC (MPT , 7), (OBSC (MPT , I) , I=9,

114 ), (OBSC (MPT , I) , I $=16,17$ )

200 FORMAT (2X,11F7.0)

OBSC (MPT , 15) $=$ OBSC (MPT , 11) +OBSC (MPT , 12) +OBSC (MPT , 13)+OBSC (MPT , 14)

GO TO 220

C $* * * * *$ "92" CARDS FOR OBSERVED DATA

210 CONTINUE

$M P T 1=M P T 1+1$

READ $(5,200) \quad(O B S C$ (MPT1,$K), K=18,21)$, OBSC (MPT1 1) ,

\&OBUNH3 (MPT1)

220 CONTINUE

IF (MPT1.GT.200) GO TO 450

IF (MPT.GT.200) GO TO 450

GO TO 30

C -

$C * * * * *$ "71" CARDS FOR INPUT OF WASTE OR TRIN CONCENTRATION OR LOADS

230 CONTINUE

IF (CHARST (2). EQ. CARD2) GO TO 250

READ $(5,240) \mathrm{N},(\operatorname{TRINC}(N, I), I=1,5),(\operatorname{TRINC}(N, I), I=9,14), \operatorname{TRINC}(N, 16)$

240 FORMAT ( $2 \mathrm{X}, \mathrm{I} 2,1 \mathrm{X}, 12 \mathrm{~F} 6.0)$

GO TO 260

C ***** "72" CARDS FOR WASTE OR TRIN INPUTS

250 CONTINUE

READ $(5,240) \mathrm{N},(\operatorname{TRINC}(\mathrm{N}, \mathrm{I}), I=17,21),(\operatorname{TRINL}(\mathrm{N}, \mathrm{I}), I=9,14)$,

\&TRINL $(\mathrm{N}, 16)$

260 CONTINUE

IF (N.GT.50) GO TO 450

GO TO 30

C-

C $* * * * * 51$ CARDS, SUBREACH ENVIRONMENTAL FACTORS

270 CONTINUE

$\operatorname{READ}(5,280) K, \operatorname{TEMP}(K), B P(K), \operatorname{SCOND}(K), B N(K), \operatorname{PNET}(K), \operatorname{RESP}(K), \operatorname{DUM} 1$,

$1 \mathrm{~K} 2(\mathrm{~K}), \operatorname{IK} 2(\mathrm{~K}), \operatorname{SFPM}(\mathrm{K}), \operatorname{SLOPE}(\mathrm{K}), \operatorname{PH}(\mathrm{K}), \operatorname{CTSVIG}(\mathrm{K})$

280 FORMAT (2X, I2 , 3F5.0, 5F6.0, I2 , F5.0, F8.0, IX, F3.1, IX, F6.0)

IF (K.GT.50) GO TO 450

$C * * * * *$ TURN ON P \&/OR R BY IPNET OPTIONS

IF (IPNET.EQ.1) RESP(K)=0.0

READCD

READCD

READCD

READCD

READCD

READCD

READCD

READCD

READCD

READCD

READCD

READCD

READCD

READCD

READCD

READCD

READCD

READCD

READCD

READCD

READCD

READCD

READCD

READCD

READCD

READCD

READCD

READCD

READCD

READCD

READCD

READCD

READCD

READCD

READCD

READCD

READCD

READCD

READCD

READCD

READCD

READCD

READCD

READCD

READCD

READCD

READCD

READCD

READCD 
APPENDIX I--LISTING OF COMPUTER CODE--Continued.

IF (IPNET.GE. 2) $\operatorname{PNET}(K)=0.0$

READCD

IF (IPNET.EQ.3) RESP(K) $=0.0$

READCD

$C * * * * *$ SLOPE COMPUTATIONS

$\operatorname{IF}(\operatorname{SLOPE}(K)$. EQ.0) $\operatorname{SLOPE}(K)=\operatorname{SFPM}(\mathrm{K}) / 5280$.

READCD

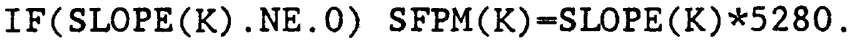

READCD

GO TO 30

READCD

READCD

C ***** "6" CARDS, SUBREACH DECAY RATES

310 CONTINUE

READ (5, 320) $\mathrm{K} 1, \mathrm{KCR}(\mathrm{K} 1), \mathrm{KC}(\mathrm{K} 1)$, DUM1, DUM1, DUM1,

\&DUM1 , KORNR (K1) , KORNF (K1) , KNH4R(K1), KNH4F(K1) , KNO2R(K1),

READCD

$\& \mathrm{KNO} 2 \mathrm{~F}(\mathrm{~K} 1), \mathrm{KNO} 3 \mathrm{R}(\mathrm{K} 1), \mathrm{DUM} 4, \mathrm{KPO} 4 \mathrm{~B}(\mathrm{~K} 1), \mathrm{DUM} 1 \quad$, KNCR1(K1),

\&KNCR2 (K1)

320 FORMAT ( $2 \mathrm{X}, \mathrm{I} 2,1 \mathrm{X}, 14 \mathrm{~F} 4.0,2 \mathrm{~F} 5.0,2 \mathrm{~F} 4.0)$

IF (K1.GT.50) GO TO 450

GO TO 30

C LINEAR DATA

C ****** "8-" CARDS - LINEAR RUNOFF DATA

330 CONTINUE

IF (CHARST (2). EQ. CARD2) GO TO 350

IF (CHARST (2).EQ.CARD3) GOTO 352

IF (CHARST (2). EQ. CARD4) GOTO 354

READCD

READCD

READCD

READCD

READCD

READCD

READCD

READCD

READCD

READCD

READCD

READCD

READCD

READCD

READCD

$\operatorname{READ}(5,340) \mathrm{N},(\operatorname{RLINC}(\mathrm{N}, \mathrm{I}), I=1,2),(\operatorname{RLINC}(\mathrm{N}, \mathrm{I}), I=4,5),(\operatorname{RLINC}(\mathrm{N}, \mathrm{I})$, $\& I=9,14),(\operatorname{RLINC}(N, I), I=16,17), \operatorname{NDIV}(\mathrm{N})$

340 FORMAT ( $2 \mathrm{X}, \mathrm{I} 2,1 \mathrm{X}, 12 \mathrm{~F} 6.0, \mathrm{I} 2)$

GO TO 370

$C * * * * * * 82 "$ CARDS

$350 \operatorname{READ}(5,360) \mathrm{N},(\operatorname{RLINC}(\mathrm{N}, \mathrm{I}), \mathrm{I}=18,21)$

360 FORMAT ( $2 \mathrm{X}, \mathrm{I} 2,1 \mathrm{X}, 12 \mathrm{~F} 6.0)$

GOTO 370

C $* * * * *$ " 83" CARDS - GROUND-WATER RETURN DATA

352 CONTINUE

$\operatorname{READ}(5,340) \mathrm{N},(\operatorname{GLINC}(N, I), I=1,2),(\operatorname{GLINC}(N, I), I=4,5),(\operatorname{GLINC}(N, I)$,

$1 \mathrm{I}=9,14),(\operatorname{GLINC}(\mathrm{N}, \mathrm{I}), \mathrm{I}=16,17)$ GO TO 370

C ****** "84" CARDS

$354 \operatorname{READ}(5,360) \mathrm{N},(\operatorname{GLINC}(\mathrm{N}, \mathrm{I}), \mathrm{I}=18,21)$

370 CONTINUE

IF (N.GT.50) GO TO 450

GO TO 30

C ******

380 ICDBUF $=1$

INITSW=1

READCD

READCD

READCD

READCD

READCD

READCD

READCD

READCD

READCD

READCD

READCD

READCD

READCD

READCD

READCD

READCD

READCD

READCD

READCD

READCD

READCD

READCD

RETURN

READCD

READCD

READCD

C ***** 31 CARDS FOR SUBREACH DESCRIPTIONS

$390 \operatorname{READ}(5,400) \mathrm{N},(\operatorname{SNAME}(\mathrm{N}, \mathrm{L}), \mathrm{L}=1,6), \operatorname{BSEG}(\mathrm{N}),(\operatorname{SDESC}(\mathrm{N}, \mathrm{LL}), \mathrm{LL}=1,11)$

READCD

400 FORMAT ( $2 \mathrm{X}, \mathrm{I} 2,1 \mathrm{X}, 6 \mathrm{~A} 4, \mathrm{~F} 6.0,1 \mathrm{X}, 11 \mathrm{~A} 4)$

READCD

GO TO 30

READCD 
APPENDIX I--LISTING OF COMPUTER CODE--Continued.

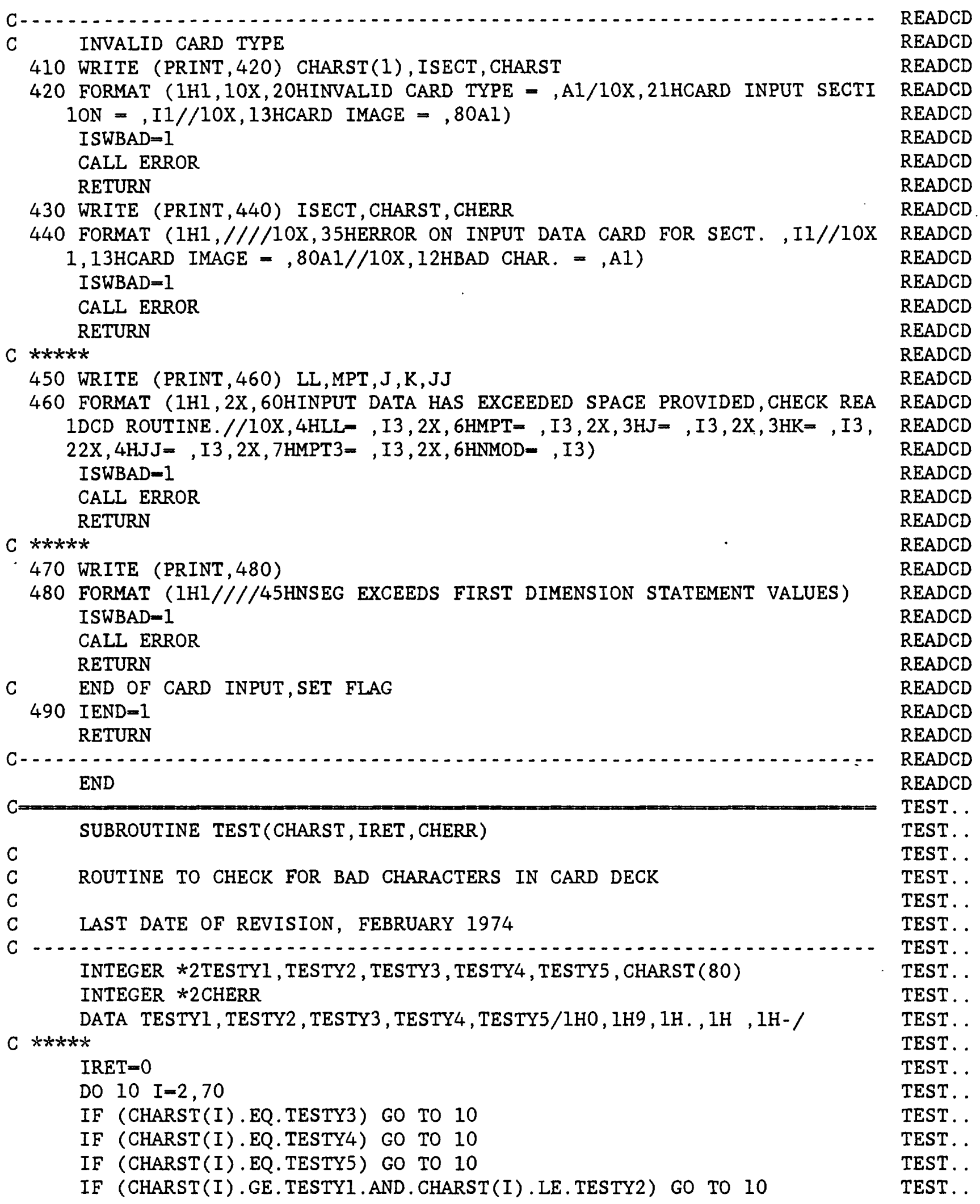


APPENDIX I--LISTING OF COMPUTER CODE--Continued.

C BAD CHAR

CHERR $=$ CHARST $(I)$

IRET $=1$

10 CONTINUE

RETURN

END

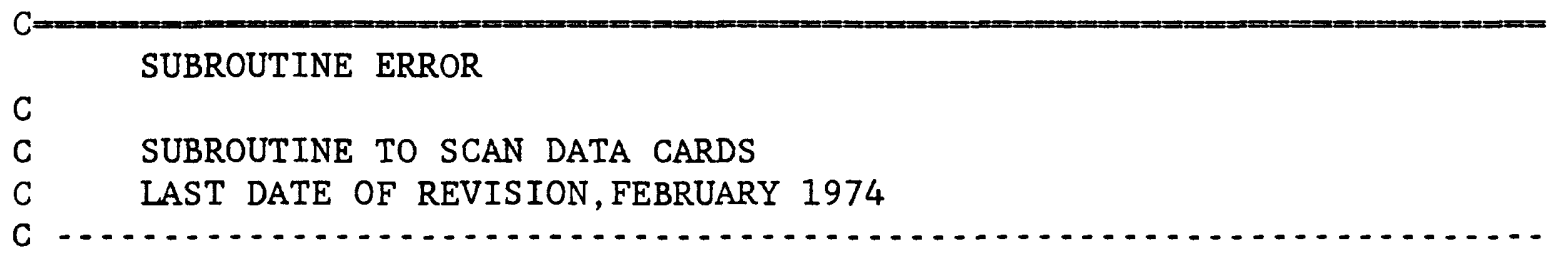

INTEGER *2CHARST $(80)$, CARD1, CARD2, CARD3, CARD4, CARD5, CARD6

INTEGER *2CARD7, CARD8, CARD9, CARDA, CARDI , CARDP , CARDK

INTEGER *2CHERR

INTEGER CARD/5/,PRINT/6/

CHARACTER $* 16$ DATE

C

DATA CARD1, CARD2, CARD3, CARD4, CARD5, CARD6, CARD7, CARD8, CARD9, CARDA, C 1ARDI , CARDP , CARDK $/ 1 \mathrm{H} 1,1 \mathrm{H} 2,1 \mathrm{H} 3,1 \mathrm{H} 4,1 \mathrm{H} 5,1 \mathrm{H} 6,1 \mathrm{H} 7,1 \mathrm{H} 8,1 \mathrm{H} 9,1 \mathrm{HA}, 1 \mathrm{HI}, 1 \mathrm{HP}, 1$ C $2 \mathrm{HK} /$

10 READ (CARD, 40, END=30) CHARST

IF (CHARST (1).EQ. CARD1) GO TO 10

IF (CHARST (1).EQ.CARDI) GO TO 10

IF (CHARST (1).EQ. CARDP) GO TO 10

IF (CHARST (1).EQ. CARD6) GO TO 10

IF (CHARST (1).EQ.CARD7) GO TO 10

IF (CHARST (1).EQ. CARDK) GO TO 10

IF (CHARST (1).EQ.CARD8) GO TO 10

CALL TEST (CHARST, IRET, CHERR)

IF (IRET.EQ.1) GO TO 20

IF (CHARST (1). EQ.CARD2) GO TO 10

IF (CHARST (1).EQ.CARD3) GO TO 10

IF (CHARST (1).EQ.CARD4) GO TO 10

IF (CHARST (1).EQ.CARD5) GO TO 10

IF (CHARST (1). EQ. CARDA) GO TO 10

IF (CHARST (1).EQ.CARD9) GO TO 10

WRITE (PRINT, 60) CHARST

GO TO 10

20 WRITE (PRINT, 50) CHARST, CHERR GO TO 10

30 RETURN

C $* * * * * *$

C $* * * * * * *$

40 FORMAT (80A1)

50 FORMAT (//10X,29HADDITION ERRORS IN INPUT DATA//10X,13HCARD IMAGE $1=, 80 \mathrm{~A} 1 / 10 \mathrm{X}, 12 \mathrm{HBAD}$ CHAR. = , A1)

60 FORMAT $(/ / 10 \mathrm{X}, 28 \mathrm{HBAD}$ CARD TYPE, CARD IMAGE $=, 80 \mathrm{~A} 1)$ END

TEST. . TEST. . TEST. . TEST. . TEST. . TEST. . ERROR. ERROR. ERROR. ERROR. ERROR . ERROR. ERROR. ERROR. ERROR. ERROR. ERROR. ERROR. ERROR. ERROR. ERROR. ERROR. ERROR. ERROR. ERROR . ERROR. ERROR. ERROR. ERROR. ERROR. ERROR. ERROR. ERROR. ERROR. ERROR. ERROR. ERROR. ERROR. ERROR. ERROR. ERROR. ERROR. ERROR. ERROR. ERROR. ERROR. ERROR. ERROR. ERROR. ERROR. 


\section{APPENDIX II--LISTING OF EXAMPLE DATA SETS}

Notes on Assumptions made for

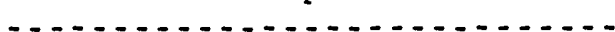

Modeling Phosphorus in the Truckee River

As discussed in detail by Nowlin (1987), model calibration and validation based on observed river data collected in 1979 and 1980 disclosed problems in mass-balance of phosphorus in the Truckee River reach from Vista to Patrick. Successful model calibration was achieved by adding calculated nonpoint loadings of phosphorus to the river in the affected reach (Nowlin, table 28, figures 30-32).

The TRWQ Model was configured to allow independent simulation of phosphorus with, and without, optional assumptions regarding potential unmeasured sources of phosphorus in the affected reach. To do this, two simulations are made for orthophosphorus calculations: orthophosphorus-A and orthophosphorus-B. Internal calculations in the model for each assume simple first-order decay or uptake, as do the calculations for total phosphorus.

In simulations presented in Nowlin (1987) and in the example data sets presented in this report, orthophosphorus-A is used to track strict accounting of orthophosphorus based on measured and estimated point and nonpoint loadings to the Truckee River. For the selected data sets with anomalous trends in observed instream concentrations, calculated nonpoint loadings of phosphorus were added as "dummy" nonpoint ground-water returns ("83,84" cards) to reaches 3 , and 7-11. For these data sets, the predicted values of orthophosphorus-B and total phosphorus provide simulations including effects of the "dummy" loadings. Calculations of the molar ratio of inorganic nitrogen to orthophosphorus use the orthophosphorus-B values.

Thus, the issue of adjustments in phosphorus loadings in the Vista to Patrick reach is handled external to the model code and at the discretion of the user. If no assumptions are made regarding "dummy" external loads of phosphorus, simulated values for orthophosphorus-A and orthophosphorus-B will be identical.

Notes on Simulations of Mininum Daily Dissolved Oxygen

As explained by Nowlin (1987), the TRWQ Model was calibrated by coding measured minimum dissolved-oxygen concentrations for all river point inputs, setting the photosynthetic production to zero, and calibrating a respiration coefficient to match observed minimum daily dissolved-oxygen concentrations. These simulations may be matched with the following model data sets by replacing mean daily dissolved-oxygen concentrations on data cards " 22 " and "71" with minimum daily concentrations and coding IPNET on card "Al" as " 0 ". 
APPENDIX II--LISTING OF EXAMPLE DATA SETS--Continued.

A. - Truckee River, June 1979

The following data set represents Truckee River conditions observed during field investigations in June 1979. Cards beginning with "C" are annotations for the following lines in the data set; they will be ignored by the TRWQ Model and are not required to run the data. Refer to table 1 for explanation of formats for input.

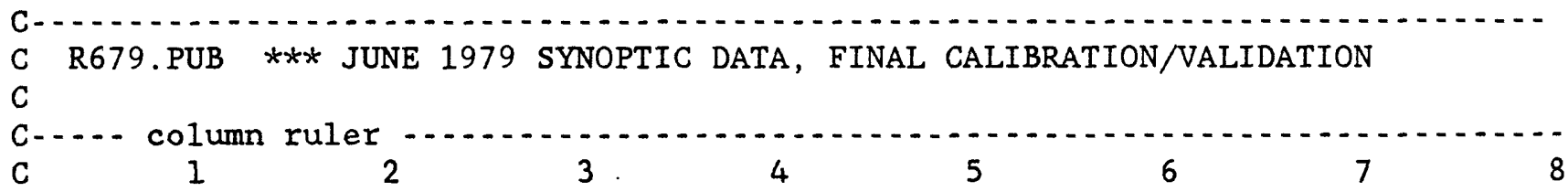

C2345678901234567890123456789012345678901234567890123456789012345678901234567890 C-1. C...- primary options: autoscale plots, daily mean DO A1 010 C -... DATA FOR 1ST SUBMODEL: NORTH TRUCKEE DRAIN 11TRUCKEE RIVER, MCCARRAN BRIDGE TO DERBY DAM 12NORTH TRUCKEE DRAIN, KLEPPE LANE TO MOUTH

C...- output formats: print all tables and plots

(R679. PUB MEAN DO)

$\begin{array}{lllllllll}21 & 00 & 00 & 01 & 02 & .1 & .26 & .00 & 0\end{array}$

C.-.- starting discharge, concentrations, etc. for condition modeled ........

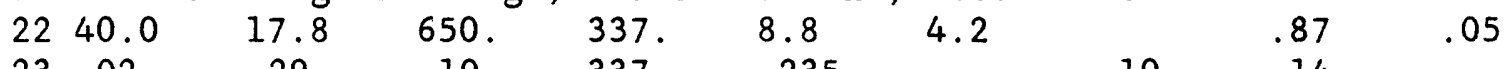

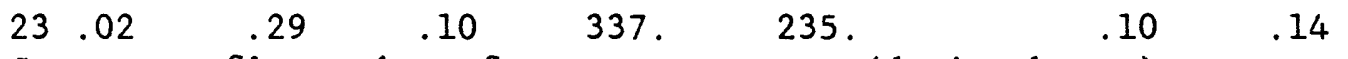

C...- configuration of stream segments (don't change)
3101 KLEPPE LANE TO MOUTH . 26 NO DIVERSIONS OR RETURNS

C...- channel geometry data (don't change)

$4101 \quad .58 \quad .26$

5.0 .30

C...- environmental data for subreachs for condition modeled

510118.0650 . 340 .

122.3

C.-.- calibrated/validated reaction rates (don't change)

6101.20 .20

$.10 .10 .40 .401 .01 .0 \quad .30$

8.5

C.-201. 20.20 (c) .25

\begin{tabular}{|c|c|c|c|c|c|c|c|c|c|c|}
\hline$\ldots$ & observec & ata & during & tield & studies & $(c a \perp i b)$ & tion & targets) & & \\
\hline 91.26 & 12.2 & 152. & 5.0 & & 1.2 & .06 & .04 & .39 & .12 & 385. \\
\hline 91.26 & 8.8 & 108. & 4.2 & & .87 & .05 & .02 & .29 & .10 & 337. \\
\hline 91.26 & 5.0 & 60 & 3.5 & & .66 & .04 & .02 & .23 & .08 & 303. \\
\hline 92268 . & & .12 & .20 & 40. & 0.00 & & & & & \\
\hline 92235. & & .10 & .14 & 40. & .005 & & & & & \\
\hline 92211. & & .08 & .12 & 35. & 0.00 & & & & & \\
\hline
\end{tabular}

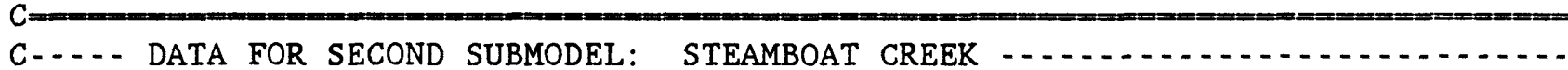

C $\ldots \ldots \ldots$

1ITRUCKEE RIVER, MCCARRAN BRIDGE TO DERBY DAM

12STEAMBOAT CREEK, KIMLICK LANE TO MOUTH

(R679.PUB MEAN DO)

C...- output formats: print all tables and plots 
APPENDIX II--LISTING OF EXAMPLE DATA SETS--Continued.

$\begin{array}{lllllllll}21 & 99 & 99 & 02 & 03 & .1 & .75 & .05\end{array}$

C....- starting discharge, concentrations, etc. for condition modeled ........

$\begin{array}{llllllll}22 & 50 . & 19.1 & 650 . & 367 . & 7.8 & 7.6 & 1.2\end{array}$

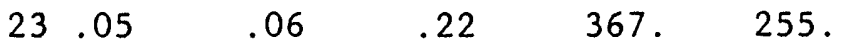

$.22 \quad .27$

C.-.- configuration of stream segments (don't change)

3101 KIMLICK LANE TO STP .75 NO DIVERSIONS OR RETURNS

3102 STP OUTLINE TO MOUTH .13 STP OUTFALL ENTERS AT HEAD

C.... channel geometry data (don't change)

4101

$.26 \quad .32$

14. .20

4102

.088 .30

14. .20

C...- environmental data for subreachs for condition modeled
510119.0650 .370 .
12.5
8.0
510220.0650 .420$.
12.5
8.5

C.... calibrated/validated reaction rates (don't change)

$6101.20 \quad .20$

$.10 .10 .40 \quad .401 .0 \quad 1.0 \quad .30$

.250

$.25 \quad .25$

61021.7 .20

$\begin{array}{lllllll}1.7 & .80 & .40 & .40 & 10.10 . & .30\end{array}$

.250

25.25

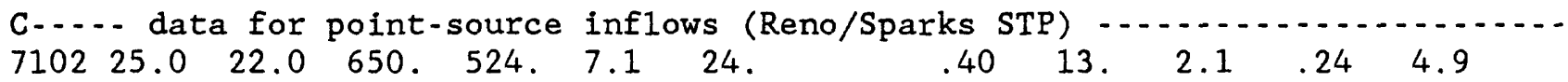

7202524.299 . $4.9 \quad 5.8$

C...- observed data during field studies (calibration targets)

\begin{tabular}{|c|c|c|c|c|c|c|c|c|c|c|}
\hline 91.75 & 10.3 & 136. & 11.0 & & 1.5 & .10 & .06 & .10 & .25 & 413 \\
\hline 91.75 & 7.8 & 99. & 7.6 & & 1.2 & .06 & .05 & .06 & .22 & 367 \\
\hline 91.75 & 4.6 & 58. & 5.5 & & .93 & .03 & .02 & .03 & .19 & 335 \\
\hline 92287. & & .25 & .30 & 60. & 0.00 & & & & & \\
\hline 92255. & & .22 & .27 & 50. & 0.00 & & & & & \\
\hline 92233. & & .19 & .24 & 40. & 0.00 & & & & & \\
\hline
\end{tabular}

C.... DATA FOR MAINSTEM TRUCKEE RIVER -

1ITRUCKEE RIVER, MCCARRAN BRIDGE TO DERBY DAM

12MAINSTEM TRUCKEE RIVER

(R679.PUB MEAN DO)

C.-.- output formats: print summary tables $8 \& 9$ and plots

$\begin{array}{lllllllll}21 & 99 & 00 & 43 & 00 & .1 & 56.12 & 0.00 & 1\end{array}$

C...- starting discharge, concentrations, etc. for condition modeled -......

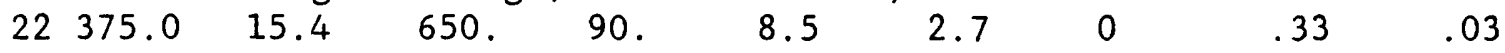

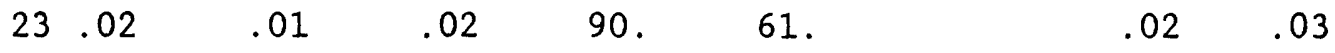

3101 MCCARRAN BR - N TRUCKEE 56.12 NO DIVERSIONS OR RETURNS

$3102 \mathrm{~N}$ TRUCKEE DR - STEAMBT 53.66 N TRUCKEE DRAIN AT HEAD

3103 STEAMBOAT - VISTA 53.53 STEAMBOAT C AT HEAD

3104 VISTA - LARGOMARSINO DV 52.23 NO DIVERSION

3105 LARGOMARSINO DIVERSIONS 51.25 NOCE AND MURPHY DIVERSIONS

3106 BELOW LARG. - LOCKWOOD 50.90 NOCE AND MURPHY RETURNS

3107 LOCKWOOD - GROTON DIV 50.05 L-NOCE \& L-MURPHY RETURNS

3108 GROTON DV - MUSTANG BR\#149.90 GROTON \& L-MURPHY RETURNS

3109 MUSTANG BR - LAST RET. 48.25 L-MURPHY RETURNS

3110 MCCARRAN POOL 46.68

3111 MCCARRAN DV - PATRICK B 46.35

3112 PATRICK BR - SP RR BR 44.92

3113 SP RR BR - HILL DIV 42.88

3114 HILL DIV - TRACY DIV 42.02

BACKWATER ABOVE MCCARRAN DAM

NO RETURNS

MCCARRAN RETURNS

BACKWATER ABOVE HILL DAM

3115 TRACY DIV - TRACY BRIDGE40.76 NO RETURNS 
APPENDIX II--LISTING OF EXAMPLE DATA SETS--Continued.

\begin{tabular}{|c|c|c|c|}
\hline 116 & TRACY BR - CLARK BR & 40.62 & HIIL RETURNS \\
\hline 117 & CLARK BR - RM 37.1 & 38.60 & NO DIVERSIONS OR RETURNS \\
\hline 18 & RM $37.1-I-80$ OXBOW & 37.10 & NO DIVERSIONS OR RETURNS \\
\hline 119 & I-80 OXBOW - DERBY DAM & 35.60 & BACKWATER ABOVE DERBY DAM \\
\hline 120 & DERBY DAM - GAGE CABLE & 34.88 & TRUCKEE CANAL DIVERTS AT HEAD \\
\hline 121 & GAGE CABLEWAY - WASHBRN & 34.52 & NO DIVERSIONS OR RETURNS MODELED \\
\hline 122 & WASHBURN -PAINTED ROCK & 31.28 & WASHBURN \& TRUCKEE CANAL RETURNS \\
\hline 123 & PAINTED R - GREGORY-MONT & $\Gamma 29.97$ & NO RETURNS \\
\hline 124 & GREGORY-MONTE - RM 28.0 & 29.35 & GREGORY-MONTEAND CANAL RETURNS \\
\hline 125 & RM 28.0 - HERMAN DIV & 28.00 & HERMAN AND CANAL RETURNS \\
\hline 126 & HERMAN DIV - PIERSON DIV & v26.75 & HERMAN AND CANAL RETURNS \\
\hline 127 & PIERSON DIV - PROCTR DIV & 25.95 & PIERSON , \& CANAL RETURNS \\
\hline 128 & PROCTOR - WADSWORTH & 23.90 & FERNLEY AREA GROUND-WATER RETURNS \\
\hline 29 & WADSWORTH -FELLNAGLE DN & 23.69 & FERNLEY AREA GROUND-WATER RETURNS \\
\hline 3130 & FELLNAGLE - RM 21.4 & 22.55 & OLINGHOUSE \#1 \& FELLNAGLE RETURNS \\
\hline 131 & RM 21.4 - S-BAR-S DIV & 21.40 & OLINGHOUSE \#1 \& FELLNAGLE RETURNS \\
\hline 3132 & S-S DAM - S-S PUMP & 19.84 & S-S AND OLINGHOUSE \#2 RETURNS \\
\hline 3133 & S-BAR-S PUMP - RM 15.8 & 17.82 & S-S DAM, S-S PUMP \& OLINGH.\#3 RETURNS \\
\hline 3134 & RM 15.8 - DEAD OX WASH & 15.82 & SALINE GROUND WATER INFLOWS \\
\hline 3135 & DEAD OX - RM 10.0 & 13.18 & SALINE GROUND WATER INFLOWS \\
\hline 3136 & $\mathrm{RM} 10.0-\mathrm{RM} 9.2$ & 10.0 & SALINE GROUND WATER INFLOWS \\
\hline 3137 & RM9.2 - NUMANA DAM & 9.20 & SALINE GROUND WATER INFLOWS \\
\hline & NUMANA DAM - RM 7.6 & 8.21 & NO RETURNS \\
\hline & $\mathrm{RM} 7.6-\mathrm{RM} 6.8$ & 7.60 & NUMANA RETURNS \\
\hline 31 & $-\mathrm{RM} 4.0$ & 6.80 & NUMANA RETURNS \\
\hline & RM 4.0 - NIXON BRIDGE & 4.00 & NUMANA RETURNS \\
\hline & NIXON BRIDGE - RM 1.0 & 3.22 & NUMANA RETURNS \\
\hline & M 1.0 - MARBLE BLUFF & & NUMANA RETURNS \\
\hline
\end{tabular}

C.... channel geometry data (don't change)

.0695 .50

50. .1

4102

.0733 .50

.0351 .603

4104

.0351 .603

54. . . 1

.0137 .765

65. . . 1

4105

.0137 .765

63. . 1

4106

.0137 .765

67. .1

4107

.0401 .634

$60 . .1$

4108

.0343 .634

60. . 1

4109

.0343 .634

4110

.0355 .634

4112

.0427 .583

4113

.0427 .583

.0280 .576

4114

.0518 .586

.0518 .586

4116

.0374 .582

65. . .

55. . 1

68. . 1

65. . . 1

56. . 1

75. . 1

76. .1

71. . 1

62. . 1

4117

.0374 .582

58. . 1

4118

.0374 .582

54. . 1

4119

.0231 .750

52. . 1

4120

.0231 .750

53. . 1

48. . 1 
APPENDIX II--LISTING OF EXAMPLE DATA SETS--Continued.

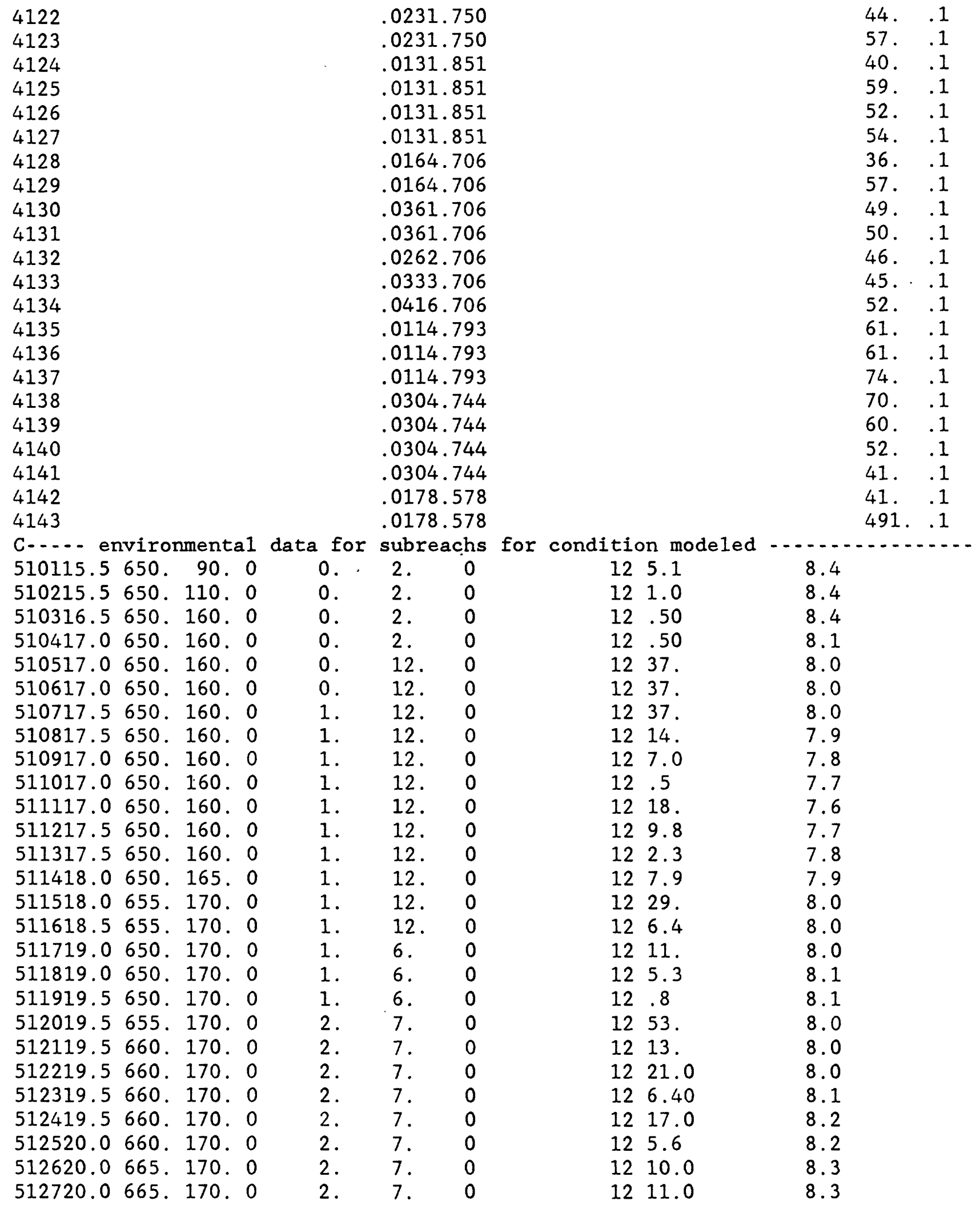




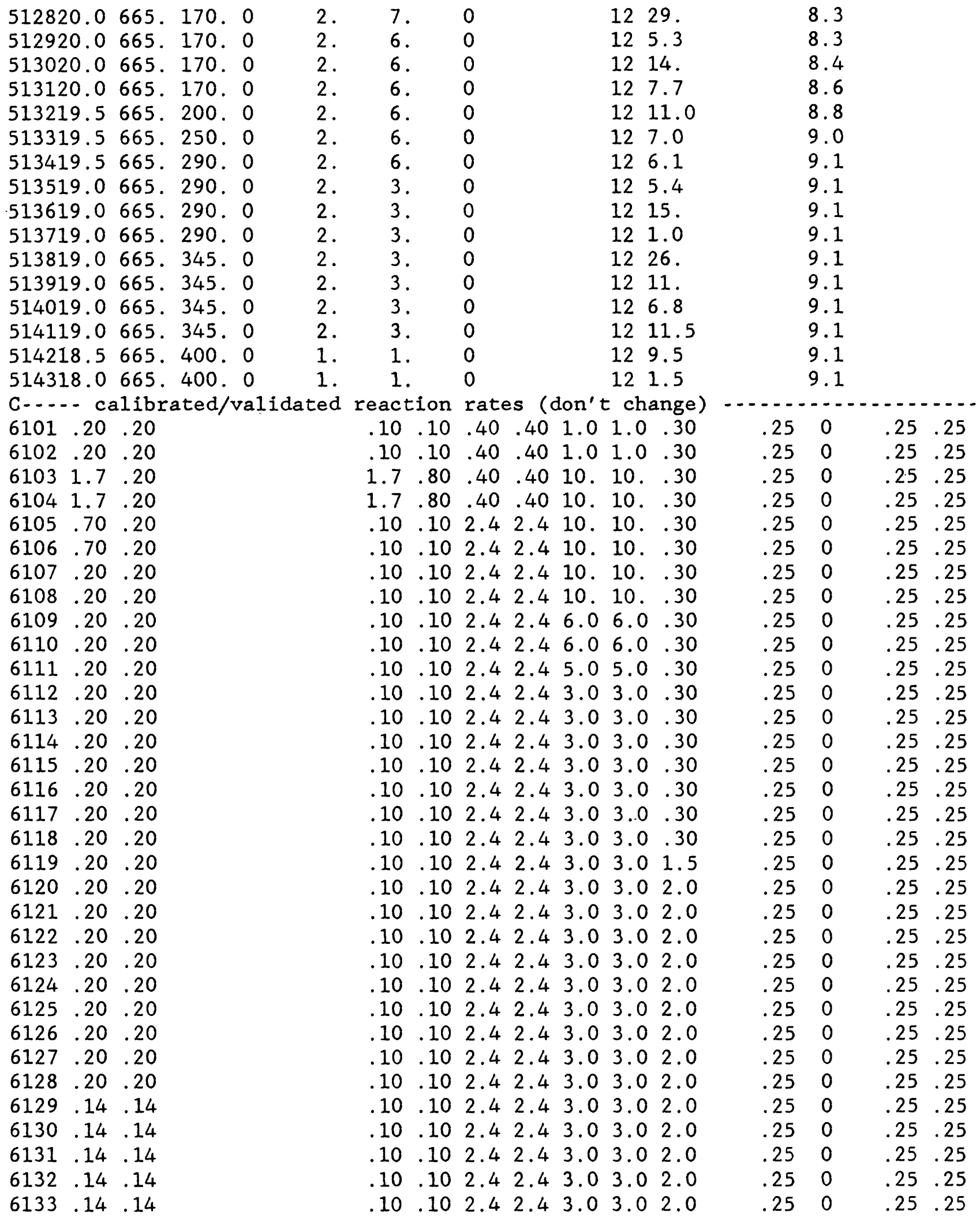


APPENDIX II--LISTING OF EXAMPLE DATA SETS--Continued.

\begin{tabular}{|c|c|c|c|c|c|c|c|c|c|c|c|c|}
\hline 6134 & \multicolumn{4}{|c|}{.14 .14} & .10 & .102 .4 & 2.4 & 3.03 .0 & 2.0 & .25 & 0 & 25.25 \\
\hline 6135 & \multicolumn{4}{|c|}{.14 .14} & .10 & .102 .4 & 2.4 & 3.03 .0 & 2.0 & .25 & 0 & .25 \\
\hline 6136 & \multicolumn{4}{|c|}{.14 .14} & .10 & 102.4 & 2.4 & 3.03 .0 & 2.0 & .25 & 0 & .25 \\
\hline 6137 & \multicolumn{4}{|c|}{.14 .14} & .10 & 102.4 & 2.4 & 3.03 .0 & 2.0 & .25 & 0 & .25 \\
\hline 6138 & \multicolumn{4}{|c|}{.14 .14} & .10 & .102 .4 & 2.4 & 3.03 .0 & 2.0 & .25 & 0 & .25 \\
\hline 6139 & \multicolumn{4}{|c|}{.14 .14} & .10 & 102.4 & 2.4 & 3.03 .0 & 2.0 & .25 & 0 & .25 \\
\hline 6140 & \multicolumn{4}{|c|}{.14 .14} & .10 & 102.4 & 2.4 & 3.03 .0 & 2.0 & .25 & 0 & $.25 \quad .25$ \\
\hline 6141 & \multicolumn{4}{|c|}{.14 .14} & .10 & .102 .4 & 2.4 & 3.03 .0 & 2.0 & .25 & 0 & $.25 \quad .25$ \\
\hline 6142 & \multicolumn{4}{|c|}{.14 .14} & .10 & .102 .4 & 2.4 & 3.03 .0 & 2.0 & .25 & 0 & .25 \\
\hline 6143 & \multicolumn{4}{|c|}{.14 .14} & .10 & .102 .4 & 2.4 & 3.03 .0 & 2.0 & .25 & 0 & $.25 \quad .25$ \\
\hline \multirow{2}{*}{\multicolumn{13}{|c|}{ burce inflows (negative discharges }} \\
\hline & \multirow{2}{*}{\multicolumn{12}{|c|}{-5.0}} \\
\hline $\begin{array}{l}7108 \\
7111\end{array}$ & & & & & & & & & & & & \\
\hline 7114 & \multirow{2}{*}{\multicolumn{12}{|c|}{-4.0}} \\
\hline 7115 & \multirow{2}{*}{\multicolumn{8}{|c|}{-4.0}} & & & & \\
\hline 7120 & \multirow{2}{*}{\multicolumn{12}{|c|}{-390 . }} \\
\hline 7122 & \multicolumn{2}{|l|}{-6.0} & & & & & & & & & & \\
\hline 7124 & -5.0 & & & & & & & & & & & \\
\hline 7126 & -11.0 & & & & & & & & & & & \\
\hline 7127 & -8.0 & & & & & & & & & & & \\
\hline 7128 & -8.0 & & & & & & & & & & & \\
\hline 7138 & -20.0 & & & & & & & & & & & \\
\hline$C \ldots$ & - da & ta $\mathrm{f}$ & SUI & n & oint & return & (se & e table & 1 fo & explan & ati & \\
\hline C & & & & & & & & of ne & ative & values & - - & $\cdots \ldots$ \\
\hline 8106 & 2.1 & 22. & 130. & -.70 & 10.0 & & 1.3 & .10 & .10 & .30 & .50 & -1.2005 \\
\hline 8107 & .7 & 22 . & 130. & -.70 & 10.0 & & 1.3 & .10 & .10 & .30 & .50 & -1.2005 \\
\hline 8108 & 14. & 22 . & 130. & -.70 & 10.0 & & 1.3 & .10 & .10 & .30 & 50 & -1.20 \\
\hline 8109 & 9.1 & 22 . & 130. & -.70 & 10.0 & & 1.3 & .10 & .10 & .30 & .50 & -1.20 \\
\hline 8111 & 2.0 & 22 . & 130. & -.70 & 10.0 & & 1.3 & .10 & .10 & .30 & .50 & -1.2000 \\
\hline 8112 & 17.7 & 22 . & 130. & -.70 & 10.0 & & 1.3 & .10 & .10 & .30 & .50 & -1.2011 \\
\hline 8114 & .4 & 22 . & 130. & -.70 & 10.0 & & 1.3 & .10 & .10 & .30 & .50 & -1.2000 \\
\hline 8115 & .2 & 22 . & 130. & -.70 & 10.0 & & 1.3 & .10 & .10 & .30 & .50 & -1.2014 \\
\hline 8116 & 2.8 & 22 . & 130. & -.70 & 10.0 & & 1.3 & .10 & .10 & .30 & .50 & -1.2014 \\
\hline 8120 & .4 & 22 . & 130. & -.70 & 10.0 & & 1.3 & .10 & .10 & .30 & .50 & -1.2000 \\
\hline 8121 & 3.6 & 22 . & 130. & -.70 & 10.0 & & 1.3 & .10 & .10 & .30 & .50 & -1.2000 \\
\hline 8122 & 3.5 & 22 . & 130. & -.70 & 10.0 & & 1.3 & .10 & .10 & .30 & .50 & -1.2000 \\
\hline 8123 & 0.7 & 22 . & 130. & -.70 & 10.0 & & 1.3 & .10 & .10 & .30 & .50 & -1.2000 \\
\hline 8124 & 2.3 & 22 . & 130. & -.70 & 10.0 & & 1.3 & .10 & .10 & .30 & .50 & -1.2000 \\
\hline 8125 & 2.2 & 22 . & 130. & -.70 & 10.0 & & 1.3 & .10 & .30 & .30 & .50 & -1.2000 \\
\hline 8126 & 1.0 & 22 . & 130. & -.70 & 10.0 & & 1.3 & .10 & .10 & .30 & .50 & -1.2000 \\
\hline 8127 & 8.2 & 22 . & 130. & -.70 & 10.0 & & 1.3 & .10 & .10 & .30 & .50 & -1.2000 \\
\hline 8128 & 1.1 & 22 . & 130. & -.70 & 10.0 & & 1.3 & .10 & .10 & .30 & .50 & $-1.20 \quad 26$ \\
\hline 8130 & .1 & 22 . & 130. & -.70 & 25.0 & & 1.3 & .10 & .10 & .30 & .50 & $-1.20 \quad 28$ \\
\hline 8131 & .4 & 22 . & 130. & -.70 & 25.0 & & 1.3 & .10 & .10 & .30 & 50 & -1.2028 \\
\hline 8132 & 1.7 & 22 . & 130. & -.70 & 25.0 & & 1.3 & .10 & .10 & .30 & .50 & $-1.20 \quad 28$ \\
\hline 8133 & 1.7 & 22 . & 130. & -.70 & 25.0 & & 1.3 & .10 & .10 & .30 & .50 & -1.2028 \\
\hline 8140 & 2.7 & 22 . & 130. & -.70 & 10.0 & & 1.3 & .10 & .10 & .30 & .50 & -1.2038 \\
\hline 8141 & .8 & 22 . & 130. & -.70 & 10.0 & & 1.3 & .10 & .10 & .30 & .50 & -1.2038 \\
\hline 8206 & -1.20 & & .50 & .60 & & & & & & & & \\
\hline
\end{tabular}


APPENDIX II--LISTING OF EXAMPLE DATA SETS--Continued.

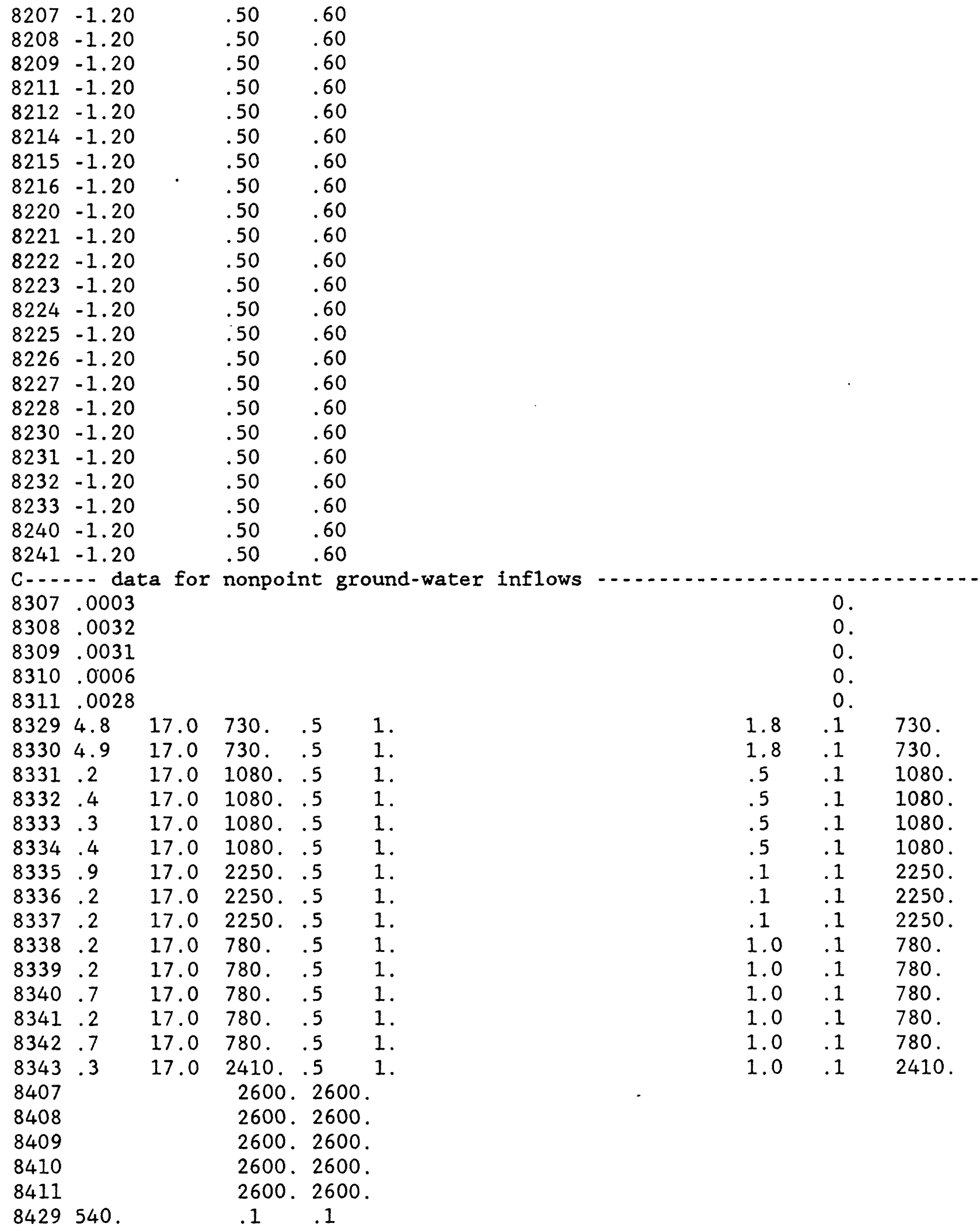


APPENDIX II--LISTING OF EXAMPLE DATA SETS--Continued.

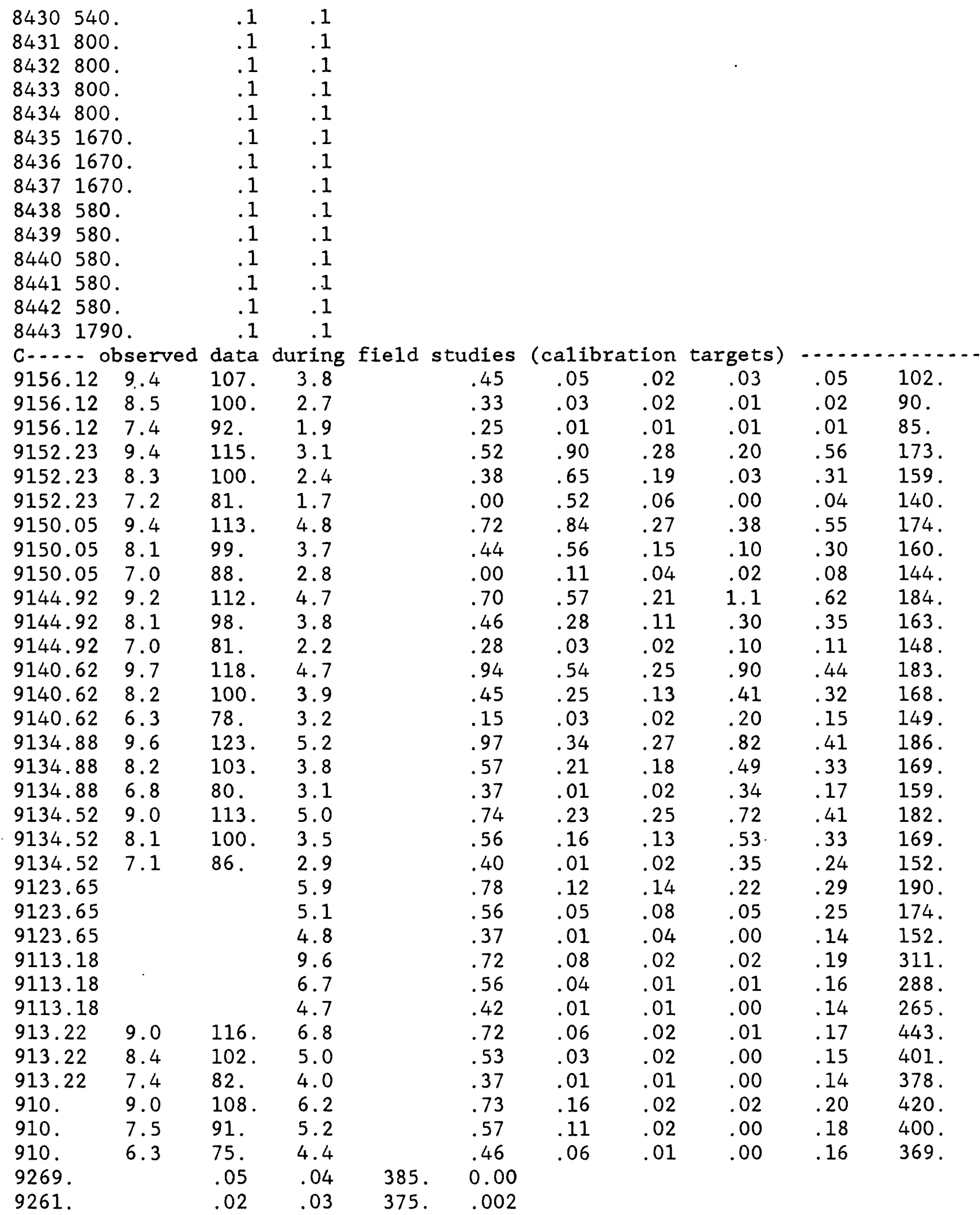


APPENDIX II--LISTING OF EXAMPLE DATA SETS--Continued.

\begin{tabular}{|c|c|c|c|c|}
\hline 9258. & .01 & .02 & 335. & 0.00 \\
\hline 92114 . & .56 & .53 & 560. & 0.00 \\
\hline 92106. & .31 & .38 & 490. & 0.00 \\
\hline 9295. & .04 & .06 & 420. & 0.00 \\
\hline 92114. & .55 & .56 & 545. & .055 \\
\hline 92107. & .30 & .36 & 470. & .018 \\
\hline 9297. & .08 & .08 & 405. & .009 \\
\hline 92120 . & .62 & .58 & 520. & .025 \\
\hline 92108. & .35 & .40 & 460. & .006 \\
\hline 92100. & .11 & .12 & 405. & .002 \\
\hline 92120. & .44 & .52 & 520. & .038 \\
\hline 92111. & .32 & .42 & 470. & .013 \\
\hline 92100. & .15 & .32 & 410. & .011 \\
\hline 92121. & .41 & .51 & 530. & .042 \\
\hline 92112. & .33 & .40 & 480 . & .012 \\
\hline 92106. & .17 & .21 & 420. & .004 \\
\hline 92119. & .41 & .47 & 110. & 0.00 \\
\hline 92112. & .33 & .38 & 90. & .005 \\
\hline 92102. & .24 & .29 & 65. & 0.00 \\
\hline 92124. & .29 & .37 & 95. & .059 \\
\hline 92114. & .25 & .32 & 75. & .025 \\
\hline 92102. & .14 & .19 & 60. & .001 \\
\hline 92192. & .19 & .29 & 110. & .050 \\
\hline 92179. & .16 & .23 & 90. & .015 \\
\hline 92166. & .14 & .18 & 75. & .003 \\
\hline 92268. & .17 & .20 & 85. & 0.00 \\
\hline 92244 . & .15 & .19 & 75. & 0.0 \\
\hline 92231. & .14 & .16 & 50. & 0.00 \\
\hline 92254. & .20 & .25 & 85. & 0.00 \\
\hline 92243. & .18 & .22 & 76. & 0.00 \\
\hline 92225 . & .16 & .20 & 50. & 0.00 \\
\hline
\end{tabular}




\section{APPENDIX II--LISTING OF EXAMPLE DATA SETS--Continued.}

B.--Truckee River, August 1979

The following data set represents Truckee River conditions observed during field investigations in August 1979. The printed output from this data set is presented in Appendix III. Format controls ("21" cards) have been set to provide the following outputs:

North Truckee Drain submode1-- all tables (full tables $8 \& 9$ ), no graphs

Steamboat Creek submodel-- all tables (full tables $8 \& 9$ ), no graphs suppresed

Mainstem river-- all tables and graphs, tables 8 and 9 limited to summary data for ends of subreaches

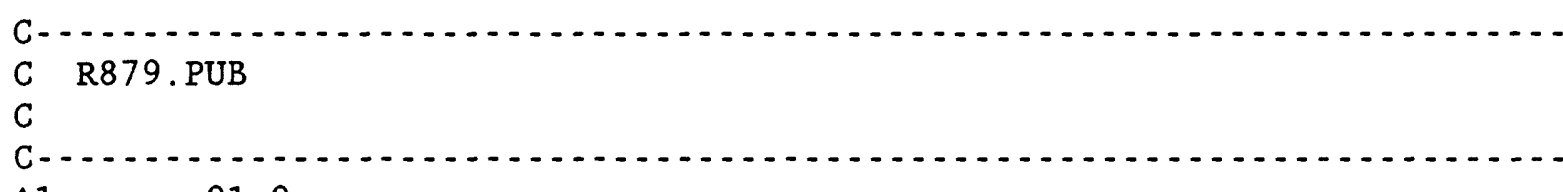

A1 010

C. - . - NORTH - TRUCKEE- DRAIN- SUBMODEL

11TRUCKEE RIVER, MCCARRAN BRIDGE TO MARBLE BLUFF DAM

12NORTH TRUCKEE DRAIN, KLEPPE LANE TO MOUTH (R879. PUB MEAN DO)
$\begin{array}{lllllllll}21 & 00 & 99 & 01 & 02 & .1 & .26 & .00\end{array}$
$\begin{array}{llllll}22 & 50.0 & 19.9 & 652 . & 359 . & 7.0\end{array}$
3.9
.68
.02
$23.01 \quad .41 \quad .11 \quad 359.250$.
$.11 \quad .10$

3101 KLEPPE LANE TO MOUTH .26 NO DIVERSIONS OR RETURNS 4101 $.58 \quad .26$

$12 \quad 2.3$

6101.20 .20

$91.26 \quad 11.0 \quad 147 . \quad 4.6$

$91.26 \quad 7.0 \quad 90 . \quad 3.9$

$91.26 \quad 4.4 \quad 56 . \quad 3.4$

$92276 . \quad .14 \quad .10$

$\begin{array}{lll}92250 . & .11 & .10 \\ 92228 & .08 & .09\end{array}$

\begin{tabular}{|c|c|c|c|c|c|c|}
\hline & & & $12 \quad 2.3$ & & 7.9 & .30 \\
\hline 10.10 & .40 & .401 .0 & 1.0 .30 & .25 & 0 & .25 .25 \\
\hline & .81 & .03 & .01 & .46 & .14 & 396. \\
\hline & .68 & .02 & .01 & .41 & .11 & 359. \\
\hline & .62 & .01 & .01 & .28 & .08 & 328. \\
\hline 52. & .006 & 3.9 & 540. & & & \\
\hline & .001 & 2.0 & 400. & & & \\
\hline & .000 & 1.3 & 320. & & & \\
\hline
\end{tabular}

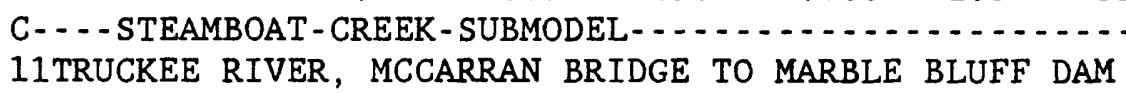

12STEAMBOAT CREEK, KIMLICK LANE TO MOUTH (R879.PUB MEAN DO)
$\begin{array}{llllllll}21 & 00 & 99 & 02 & 03 & .1 & .75\end{array}$
$\begin{array}{llllll}22 & 40.0 & 22.2 & 652 . & 279 . & 5.8\end{array}$
6.9
.90
$.21 \quad .24$
.10
23.01
.21
3101 KIMLICK LANE TO STP
279. 194.
.75 NO DIVERSIONS OR RETURNS
3102 STP OUTFALL TO MOUTH
4101
.13 STP OUTFALL ENTERS AT HEAD
$.26 \quad .32$
.088 .30
14. .20
14. .20 
APPENDIX II--LISTING OF EXAMPLE DATA SETS--Continued.

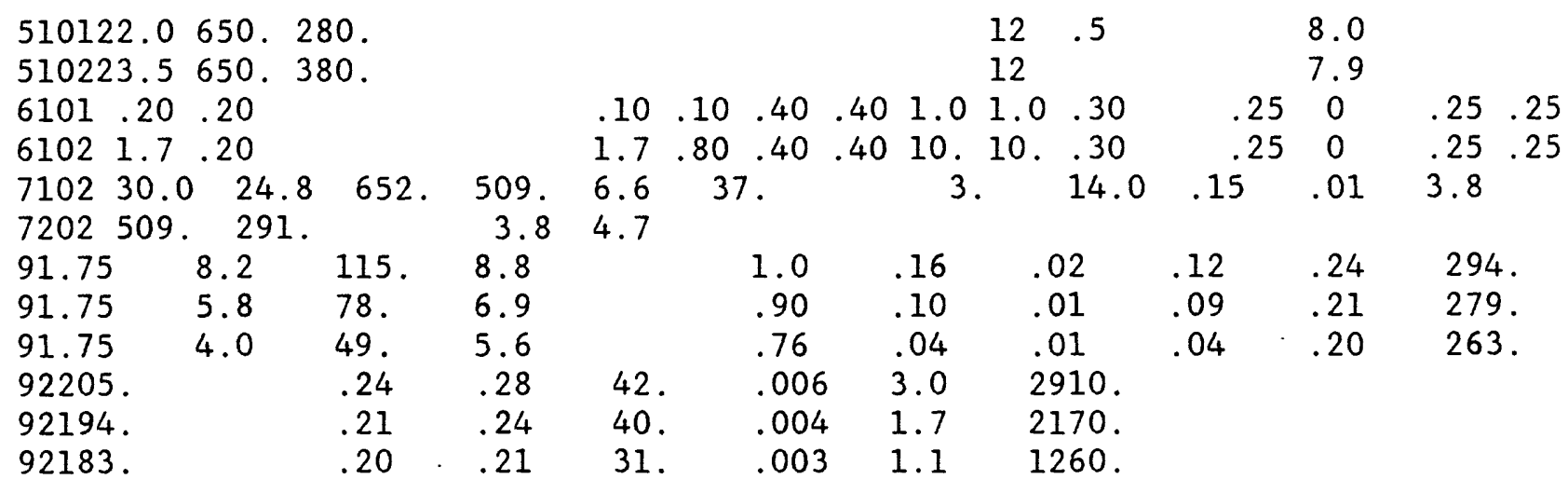

C - - - MAINSTEM-TRUCKEE-RIVER -

IITRUCKEE RIVER, MCCARRAN BRIDGE TO MARBLE BLUFF DAM

12 MAINSTEM TRUCKEE RIVER

$\begin{array}{lllllll}21 & 05 & 00 & 43 & 00 & .1 & 56.12\end{array}$

$\begin{array}{lllll}22 & 160.0 & 20.3 & 653 . & 127 .\end{array}$

$\begin{array}{lllll}23 & .01 & .04 & .08 & 127 .\end{array}$

3101 MCCARRAN BR - N TRUCKEE 56.12

3102 N TRUCKEE DR - STEAMBT 53.66

3103 STEAMBOAT - VISTA 53.53

3104 VISTA - LARGOMARSINO DV 52.23

3105 LARGOMARSINO DIVERSIONS 51.25

3106 BELOW LARG. - LOCKWOOD 50.90

3107 LOCKWOOD - GROTON DIV 50.05

3108 GROTON DV - MUSTANG BR\#149.90

3109 MUSTANG BR - LAST RET. 48.25

3110 MCCARRAN POOL 46.68

3111 MCCARRAN DV - PATRICK B 46.35

3112 PATRICK BR - SP RR BR 44.92

3113 SP RR BR - HILL DIV 42.88

3114 HILL DIV - TRACY DIV 42.02

3115 TRACY DIV - TRACY BRIDGE40.76

3116 TRACY BR - CLARK BR 40.62

3117 CLARK BR - RM $37.1 \quad 38.60$

3118 RM 37.1 - I-80 OXBOW 37.10

3119 I-80 OXBOW - DERBY DAM 35.60

3120 DERBY DAM - GAGE CABLE 34.88

3121 GAGE CABLEWAY - WASHBRN 34.52

3122 WASHBURN -PAINTED ROCK 31.28

3123 PAINTED R - GREGORY-MONT29.97

3124 GREGORY-MONTE - RM 28.029 .35

3125 RM 28.0 - HERMAN DIV 28.00

3126 HERMAN DIV - PIERSON DIV26.75

3127 PIERSON DIV - PROCTR DIV25.95

3128 PROCTOR - WADSWORTH 23.90

3129 WADSWORTH -FELLNAGLE DN 23.69

3130 FELLNAGLE - RM $21.4 \quad 22.55$

3131 RM 21.4 - S-BAR-S DIV 21.40

3132 S-S DAM - S-S PUMP 19.84

(R879.PUB MEAN DO)

$\begin{array}{llll}7.6 & 2.4 & .33 & .03\end{array}$

$86 . \quad .08 \quad .04$

NO DIVERSIONS OR RETURNS

$N$ TRUCKEE DRAIN AT HEAD

STEAMBOAT C AT HEAD

NO DIVERSION

NOCE AND MURPHY DIVERSIONS

NOCE AND MURPHY RETURNS

L-NOCE \& L-MURPHY RETURNS

GROTON \& L-MURPHY RETURNS

L-MURPHY RETURNS

BACKWATER ABOVE MCCARRAN DAM

NO RETURNS

MCCARRAN RETURNS

BACKWATER ABOVE HILL DAM

NO RETURNS

NO RETURNS

HILL RETURNS

NO DIVERSIONS OR RETURNS

NO DIVERSIONS OR RETURNS

BACKWATER ABOVE DERBY DAM

TRUCKEE CANAL DIVERTS AT HEAD

NO DIVERSIONS OR RETURNS MODELED

WASHBURN \& TRUCKEE CANAL RETURNS

NO RETURNS

GREGORY-MONTEAND CANAL RETURNS

HERMAN AND CANAL RETURNS

HERMAN AND CANAL RETURNS

HERMAN, PIERSON , \& CANAL RETURNS

FERNLEY AREA GROUND-WATER RETURNS

FERNLEY AREA GROUND-WATER RETURNS

OLINGHOUSE \#1 \& FELLNAGLE RETURNS

OLINGHOUSE \#1 \& FELLNAGLE RETURNS

$S$-S AND OLINGHOUSE \#2 RETURNS 


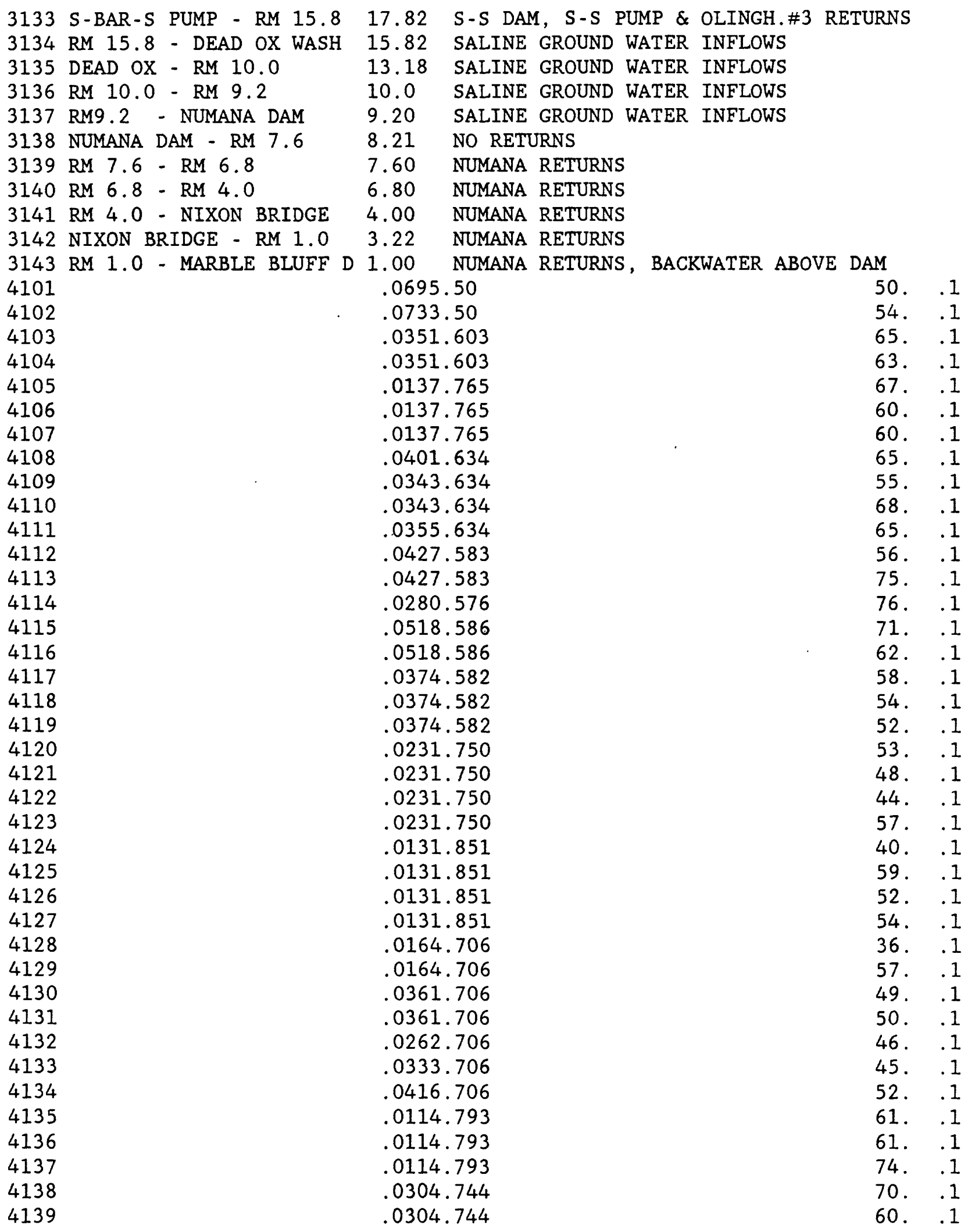


APPENDIX II--LISTING OF EXAMPLE DATA SETS--Continued.

\begin{tabular}{|c|c|c|}
\hline \multirow{2}{*}{\multicolumn{3}{|c|}{4140}} \\
\hline & & \\
\hline $\begin{array}{l}4141 \\
4142\end{array}$ & & \\
\hline $\begin{array}{l}4142 \\
4143\end{array}$ & & \\
\hline 510120.5 & 655. & 130.0 \\
\hline 510220.0 & 655. & 180. \\
\hline 510321.0 & 655. & 240 . \\
\hline 10421.0 & 655. & 240 . \\
\hline 10521.0 & 655. & 240 . \\
\hline 10621.5 & 655. & 240. \\
\hline 510721.5 & 655. & 240 . \\
\hline 510821.5 & 655. & 240. 0 \\
\hline 510921.5 & 655. & 240.0 \\
\hline 511021.5 & 655. & 240 \\
\hline 511121.5 & 655. & 240 \\
\hline 511222.0 & 655. & 240 . \\
\hline 511322.0 & 655. & 240 . \\
\hline 511422.0 & 655. & 240 . \\
\hline 511522.0 & 655 . & 250 . \\
\hline 1622.0 & 655. & 250 . \\
\hline 511722.0 & 655. & 240 . \\
\hline 1822.5 & 655. & 240 . \\
\hline 511922.5 & 655. & 240 . \\
\hline 512023.0 & 655. & 240 . \\
\hline 512123.0 & 655 . & 240 \\
\hline 512223.0 & 655. & 540. \\
\hline 512323.0 & 660. & 540. \\
\hline 512423.0 & 660. & 250. \\
\hline 512523.0 & 660. & 250. \\
\hline 512623.0 & 660. & 250 . \\
\hline 512723.0 & 660. & 260 . \\
\hline 512823.0 & 660. & 260. \\
\hline 512923.0 & 660. & 260. \\
\hline 513023.0 & 660. & 300. \\
\hline 513123.5 & 660. & 300. \\
\hline 513224.0 & 660. & 400. \\
\hline 513324.0 & 660. & 460. \\
\hline 513424.5 & 660 . & 460. \\
\hline 513524.5 & 660. & 460. \\
\hline 513624.5 & 660. & 460. \\
\hline 513724.5 & 660 . & 460. \\
\hline 513825.0 & 660. & 610. \\
\hline 513925.0 & 665 & 610. \\
\hline 514025.0 & 665 . & 610. \\
\hline 514125.0 & 665 . & 610. \\
\hline 514224.0 & 665. & 630. \\
\hline 514323.0 & 665. & 630. \\
\hline 6101.20 & .20 & \\
\hline 20 & .20 & \\
\hline $6103 \quad 1.7$ & .20 & \\
\hline
\end{tabular}

.0304 .744

.0304 .744

.0178 .578

.0178 .578

0. 2 . 0

0 2. 0

0.2 . 0

0.2 . 0

$0 . \quad 12.0$

0.12 . 0

1. 12. 0

1. 12. 0

1. 12 . 0

1. 12. 0

1. 12. 0

1. 12. 0

1. 12. 0

1. 12. 0

1. 12. 0

1. 12. 0

1. 6.0

1. 6. 0

1. 6.0

2. 7.0

2. 7.0

2. 7.0

2. 7.0

2. 7.0

2. 7.0

2. 7.0

2. 7.0

2. 7.0

2. 6. 0

2. 6. 0

2. 6. 0

2. 6. 0

2. 6.0

2. 6. 0

2. 3. 0

2. 3. 0

2. 3.0

2. 3. 0

2. 3.0

2 . 3. 0

2. $3 . \quad 0$

1. 1.0

1. 1.10

$\begin{array}{llllllll}.10 & .10 & .40 & .40 & 1.0 & 1.0 & .30\end{array}$

$\begin{array}{llllllll}.10 & .10 & .40 & .40 & 1.0 & 1.0 & .30\end{array}$

$\begin{array}{lllllll}1.7 & .80 & .40 & .40 & 10.10 . & .30\end{array}$
52. . . 1

41. . 1

41. . 1

491. . 1

8.0

8.2

8.0

7.7

7.6

7.5

7.4

7.5

7.6

7.7

7.8

7.8

7.8

7.8

7.8

7.8

7.8

7.9

8.0

8.0

8.0

8.0

8.0

8.1

8.1

8.1

8.1

8.1

8.1

8.2

8.2

8.3

8.4

8.4

8.4

8.4

8.4

8.4

8.4

8.4

8.4

8.6

8.8

.250

.250

$.25 \quad .25$

.25 .25

.25 .25 
APPENDIX II--IISTING OF EXAMPLE DATA SETS--Continued.

$\begin{array}{lll}6104 & 1.7 & .20 \\ 6105 & .70 & .20 \\ 6106 & .70 & .20 \\ 6107 & .20 & .20 \\ 6108 & .20 & .20 \\ 6109 & .20 & .20 \\ 6110 & .20 & .20 \\ 6111 & .20 & .20 \\ 6112 & .20 & .20 \\ 6113 & .20 & .20 \\ 6114 & .20 & .20 \\ 6115 & .20 & .20 \\ 6116 & .20 & .20 \\ 6117 & .20 & .20 \\ 6118 & .20 & .20 \\ 6119 & .20 & .20 \\ 6120 & .20 & .20 \\ 6121 & .20 & .20 \\ 6122 & .20 & .20 \\ 6123 & .20 & .20 \\ 6124 & .20 & .20 \\ 6125 & .20 & .20 \\ 6126 & .20 & .20 \\ 6127 & .20 & .20 \\ 6128 & .20 & .20 \\ 6129 & .14 & .14 \\ 6130 & .14 & .14 \\ 6131 & .14 & .14 \\ 6132 & .14 & .14 \\ 6133 & .14 & .14 \\ 6134 & .14 & .14 \\ 6135 & .14 & .14 \\ 6136 & .14 & .14 \\ 6137 & .14 & .14 \\ 6138 & .14 & .14 \\ 6139 & .14 & .14 \\ 6140 & .14 & .14 \\ 6141 & .14 & .14 \\ 6142 & .14 & .14 \\ 6143 & .14 & .14 \\ 7105 & -27 & . \\ 7108 & -4.0 \\ 7111 & -13.0 \\ 7114 & -6.0 \\ 7115 & -4.0 \\ 7120 & -220 . \\ 7122 & -2.0 \\ 7124 & -8.0 \\ 7126 & -14 \\ 7130 & -6.0 \\ & .0 \\ & & \\ 61 & \end{array}$


APPENDIX II--LISTING OF EXAMPLE DATA SETS--Continued.

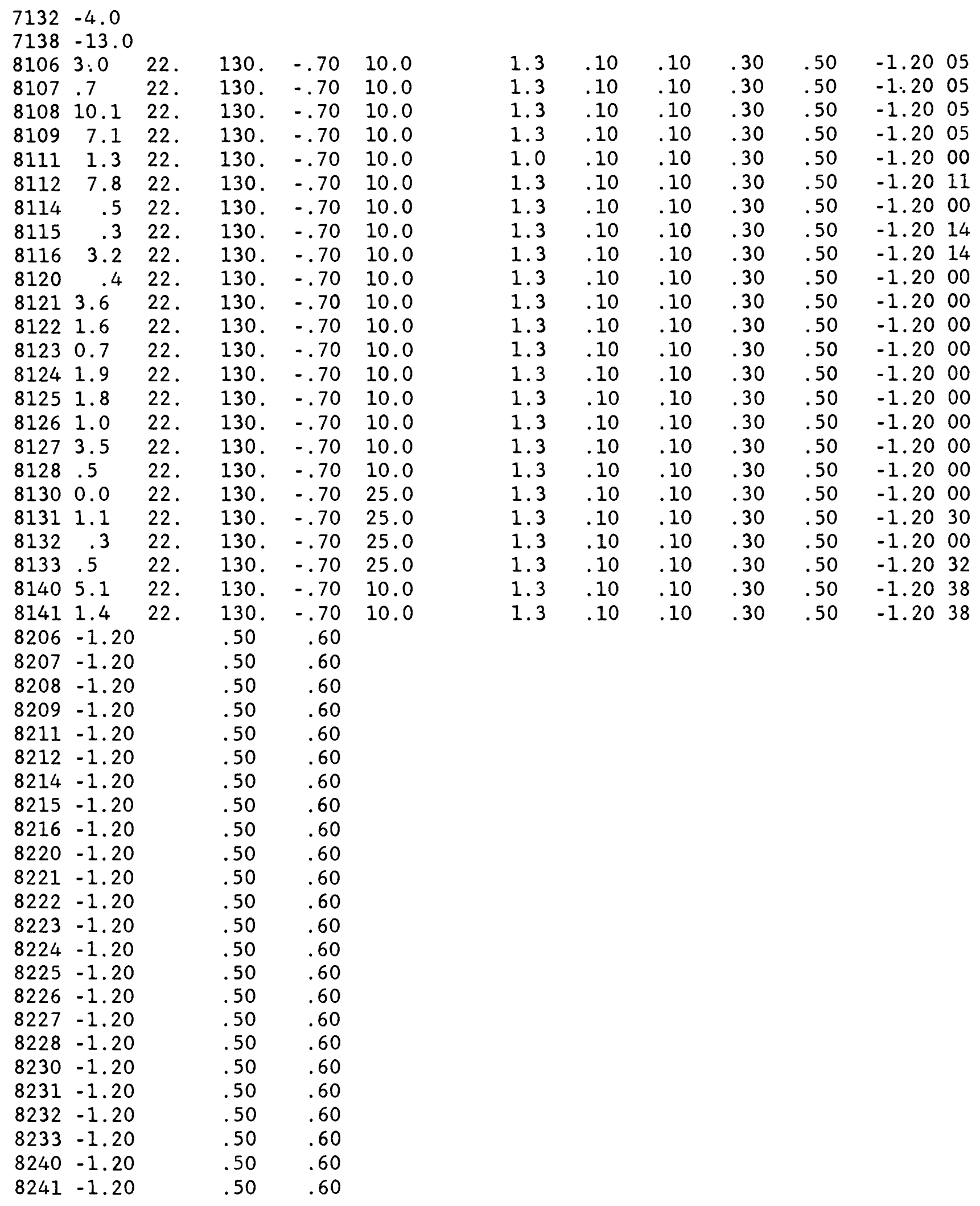




\section{APPENDIX II--LISTING OF EXAMPLE DATA SETS--Continued.}

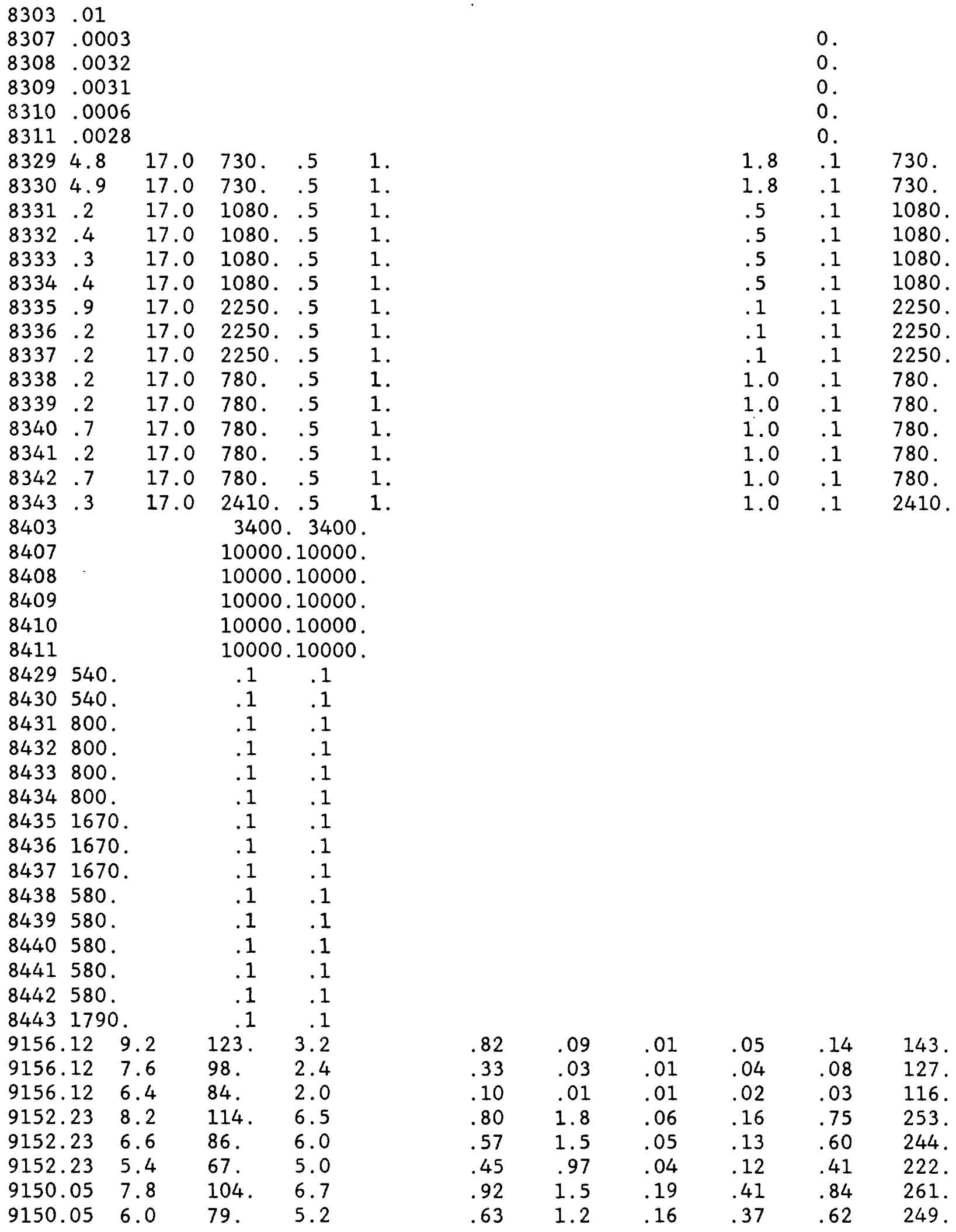


APPENDIX II--LISTING OF EXAMPLE DATA SETS--Continued.

\begin{tabular}{|c|c|c|c|c|c|c|c|c|c|c|}
\hline 9150.05 & 4.5 & 58. & 4.4 & & .45 & .79 & .10 & .31 & .42 & 230. \\
\hline 9144.92 & 9.0 & 125. & 6.2 & & .90 & .94 & .33 & .82 & 1.4 & 258. \\
\hline 9144.92 & 6.6 & 88. & 5.5 & & .70 & .58 & .28 & .77 & .91 & 243. \\
\hline 9144.92 & 4.5 & 57. & 4.4 & & .46 & .33 & .21 & .68 & .44 & 229. \\
\hline 9138.60 & 9.1 & 123. & 7.1 & & 1.1 & .32 & .33 & 1.4 & 1.1 & 268. \\
\hline 9138.60 & 6.3 & 84. & 5.3 & & .64 & .21 & .26 & 1.1 & .74 & 251. \\
\hline 9138.60 & 3.8 & 48. & 3.9 & & .45 & .10 & .16 & .92 & .47 & 241. \\
\hline 9134.88 & 10.2 & 144. & 5.4 & & .92 & .28 & .27 & 1.20 & .98 & 248. \\
\hline 9134.88 & 6.3 & 86. & 4.4 & & .68 & .11 & .19 & 1.1 & .69 & 237. \\
\hline 9134.88 & 4.4 & 58. & 3.8 & & .48 & .01 & .14 & .95 & .52 & 223. \\
\hline 9134.52 & 8.2 & 114. & 4.5 & & .75 & .23 & .23 & 1.20 & 1.1 & 246 . \\
\hline 9134.52 & 6.6 & 89. & 3.8 & & .58 & .12 & .17 & 1.10 & .72 & 238. \\
\hline 9134.52 & 5.5 & 73. & 2.9 & & .42 & .06 & .13 & .97 & .51 & 225. \\
\hline 9123.65 & 11.8 & 166. & 5.4 & & .70 & .04 & .01 & .08 & .44 & 278. \\
\hline 9123.65 & 6.9 & 94. & 4.1 & & .52 & .02 & .01 & .02 & .38 & 260 . \\
\hline 9123.65 & 3.4 & 45. & 2.9 & & .36 & .01 & .01 & .00 & .35 & 249. \\
\hline 9113.18 & 10.4 & 153. & 4.3 & & .54 & .07 & .01 & .00 & .24 & 477. \\
\hline 9113.18 & 7.2 & 100. & 3.7 & & .40 & .02 & .01 & .00 & .23 & 455. \\
\hline 9113.18 & 4.6 & 58. & 3.1 & & .27 & .01 & .01 & .00 & .22 & 431. \\
\hline 913.22 & 10.4 & 158. & 4.3 & & .99 & .01 & .01 & .03 & .20 & 649. \\
\hline 913.22 & 7.7 & 107. & 3.6 & & .66 & .01 & .01 & .01 & .14 & 607. \\
\hline 913.22 & 5.2 & 68. & 2.8 & & .38 & .01 & .01 & .00 & .11 & 564. \\
\hline 910. & 10.2 & 140 & 8.1 & & .74 & .03 & .01 & .00 & .25 & 664. \\
\hline 910. & 8.2 & 109. & 5.9 & & .62 & .02 & .01 & .00 & .23 & 634. \\
\hline 910. & 6.6 & 87. & 3.6 & 0 & .54 & .01 & .01 & .00 & .20 & 577. \\
\hline 9297. & & .14 & .11 & 175. & .035 & 2.4 & 490. & & & \\
\hline 9286. & & .08 & .04 & 160. & .002 & 1.2 & 360 . & & & \\
\hline 9279. & & .03 & .01 & 150. & .000 & .26 & 260 . & & & \\
\hline 92159. & & .75 & 1.1 & 290. & .16 & 1.1 & & & & \\
\hline 92154. & & .60 & .75 & 275 . & .049 & .53 & & & & \\
\hline 92142 . & & .41 & .46 & 260 . & .014 & .26 & & & & \\
\hline 92164 & & .84 & .87 & 270 . & .04 & 2.8 & 720 . & & & \\
\hline 92157. & & .62 & .63 & 255. & .016 & 1.5 & 610. & & & \\
\hline 92146. & & .42 & .45 & 240. & .007 & .53 & 510. & & & \\
\hline 92162. & & 1.4 & 1.4 & 280. & .075 & 2.1 & & & & \\
\hline 92154. & & .91 & .94 & 260. & .02 & 1.1 & & & & \\
\hline 92146. & & .44 & .50 & 230. & .007 & .53 & & & & \\
\hline 92168 & & 1.1 & 1.2 & 270 . & .036 & 4.1 & & & & \\
\hline 92158. & & .74 & .85 & 260. & .007 & 1.4 & & & & \\
\hline 92153. & & .47 & .60 & 230 . & .001 & .21 & & & & \\
\hline 92157. & & .98 & 1.1 & 280 . & .013 & 2.1 & 1180. & & & \\
\hline 92150. & & .69 & .78 & 260. & .006 & 1.4 & 1030. & & & \\
\hline 92142 . & & .52 & .58 & 225 . & .001 & .65 & 710 & & & \\
\hline 92155. & & 1.1 & 1.1 & 40 & .013 & 2.0 & & & & \\
\hline 92151. & & .72 & .78 & 40. & .007 & 1.0 & & & & \\
\hline 92144. & & .51 & .56 & 40. & .002 & .26 & & & & \\
\hline 92174 . & & .44 & .50 & 45. & .019 & 4.2 & & & & \\
\hline 92163. & & .38 & .48 & 31. & .002 & 1.3 & & & & \\
\hline 92157. & & .35 & .45 & 25. & .000 & .45 & & & & \\
\hline 92287 . & & .24 & .29 & 40 & .007 & 2.3 & & & & \\
\hline
\end{tabular}


APPENDIX II--LISTING OF EXAMPLE DATA SETS--Continued.

$\begin{array}{llllllll}92274 . & .23 & .25 & 35 . & .003 & 1.5 & \\ 92261 . & .22 & .22 & 30 . & .001 & .53 & \\ 92385 . & .20 & .19 & 35 . & .004 & 2.8 & \\ 92361 . & .14 & .15 & 30 . & .002 & 2.2 & \\ 92336 . & .11 & .12 & 25 . & .001 & 1.5 & \\ 92393 . & .25 & .29 & 35 . & .012 & 9.7 & 7260 . \\ 92376 . & .23 & .28 & 31 . & .007 & 6.4 & 4820 . \\ 92344 . & .20 & .24 & 25 . & .002 & 4.2 & 3350 .\end{array}$

C.......... 
APPENDIX II--LISTING OF EXAMPLE DATA SETS--Continued.

C. - Truckee River, June 1980

The following data set represents Truckee River conditions observed during field investigations in June 1980.

$\mathrm{C}$

C R680.PUB

A1 010

11TRUCKEE RIVER, MCCARRAN BRIDGE TO DERBY DAM

12NORTH TRUCKEE DRAIN, KLEPPE LANE TO MOUTH

(R680.PUB MEAN DO)

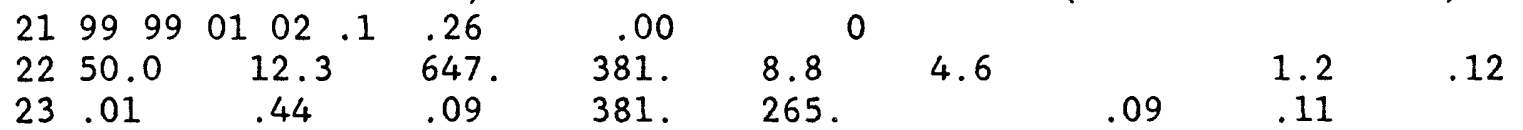

3101 KLEPPE LANE TO MOUTH .26 NO DIVERSIONS OR RETURNS

4101 $.58 \quad .26$

510112.5645 .380 .

$6101.20 \quad 20$

$\begin{array}{lllllll}91.26 & 10.3 & 120 . & 5.1 & & 1.7 & .23 \\ 91.26 & 8.8 & 98 . & 4.6 & & 1.2 & .12 \\ 91.26 & 7.2 & 77 . & 3.7 & & .80 & .00 \\ 92283 . & & .12 & .15 & 50 . & .017 & 1 . \\ 92265 . & & .09 & .11 & 50 . & .004 & 1 . \\ 92239 . & & .05 & .08 & 45 . & 0.00 & .85 \\ \text { 11TRUCKEE RIVER, } & \text { MCCARRAN BRIDGE TO DERBY DAM }\end{array}$

122.3

$\begin{array}{llllllll}.10 & .10 \quad .40 \quad .40 \quad 1.0 & 1.0 \quad .30\end{array}$

8.1

$5.0 \quad .30$

12STEAMBOAT CREEK, KIMLICK LANE TO MOUTH

$\begin{array}{lllllllll}21 & 99 & 99 & 02 & 03 & 1 & .75 & 00 & 0\end{array}$

$\begin{array}{llllllll}22 & 145 . & 13.1 & 648 . & 485 & 7.9 & 5.7 & 1.4\end{array}$

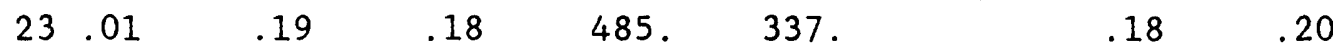

3101 KIMLICK LANE TO STP .75 NO DIVERSIONS OR RETURNS

3102 STP OUTFALL TO MOUTH .13 STP OUTFALL ENTERS AT HEAD

4101

$.26 \quad .32$

4102 $.088 \quad .30$

510113.0650 .480 .00

510214.0650 .490 .0

$\begin{array}{lll}0 & 0 & 0\end{array}$

12.5

.01

.62

44

250

.25 .25

$6101.20 \quad .20$

$10 \quad 10-40 \quad .40 \quad 1.01 .0$

12.5

.09

406.

381 .

344 .

$\begin{array}{llll}6102 & 1.7 & .20\end{array}$

$710245.0 \quad 18.6 \quad 648.498$.

$\begin{array}{lllllll}1.7 & .80 & .40 & .40 & 10.10 .30\end{array}$

$7202498.284 .4 .5 \quad 5.7$

$\begin{array}{llll}91.75 & 8.8 & 105.6 .6\end{array}$

$\begin{array}{llll}91.75 & 7.9 & 88 . & 5.7\end{array}$

$\begin{array}{llll}91.75 & 6.7 & 71 . & 4.6\end{array}$

92367.

$.20 \quad .22$

.22

$\begin{array}{ll}.18 & .20 \\ .16 & .15\end{array}$

8.635 .

6.

14.0

14. .20

14. .20

92337.

$.15125 . \quad .002$

165. .007

$\begin{array}{lll}1.6 & .26 \quad .02\end{array}$

.01

$.25 \quad .20$

8.0

7.9

92310 . $.16 \quad .15 \quad 125$.

1ITRUCKEE RIVER, MCCARRAN BRIDGE TO DERBY DAM

12MAINSTEM TRUCKEE RIVER

$\begin{array}{lllllll}21 & 99 & 05 & 43 & 00 & .1 & 56.12\end{array}$

.00

0

(R680.PUB MEAN DO) 
APPENDIX II--LISTING OF EXAMPLE DATA SETS--Continued.

$\begin{array}{llllll}22 & 1780 . & 10.3 & 648 . & 70 \\ 23.00 & .24 & .04 & 70 . & 47\end{array}$

3101 MCCARRAN BR - N TRUCKEE 56.12

3102 N TRUCKEE DR - STEAMBT 53.66

3103 STEAMBOAT - VISTA 53.53

3104 VISTA - LARGOMARSINO DV 52.23

3105 LARGOMARSINO DIVERSIONS 51.25

3106 BELOW LARG. - LOCKWOOD 50.90

3107 LOCKWOOD - GROTON DIV 50.05

3108 GROTON DV - MUSTANG BR\#149.90

3109 MUSTANG BR - LAST RET. 48.25

3110 MCCARRAN POOL 46.68

3111 MCCARRAN DV - PATRICK B 46.35

3112 PATRICK BR - SP RR BR 44.92

3113 SP RR BR - HILL DIV 42.88

3114 HILL DIV - TRACY DIV 42.02

3115 TRACY DIV - TRACY BRIDGE40.76

3116 TRACY BR - CLARK BR 40.62

3117 CLARK BR - RM $37.1 \quad 38.60$

3118 RM 37.1 - I-80 OXBOW 37.10

3119 I - 80 OXBOW - DERBY DAM 35.60

3120 DERBY DAM - GAGE CABLE 34.88

3121 GAGE CABLEWAY - WASHBRN 34.52

3122 WASHBURN -PAINTED ROCK 31.28

3123 PAINTED R - GREGORY-MONT29.97

3124 GREGORY-MONTE - RM 28.029 .35

3125 RM 28.0 - HERMAN DIV 28.00

3126 HERMAN DIV - PIERSON DIV26.75

3127 PIERSON DIV - PROCTR DIV25.95

3128 PROCTOR - WADSWORTH 23.90

3129 WADSWORTH -FELLNAGLE DN 23.69

3130 FELLNAGLE - RM $21.4 \quad 22.55$

3131 RM 21.4 - S-BAR-S DIV 21.40

3132 S-S DAM - S-S PUMP 19.84

3133 S-BAR-S PUMP - RM 15.817 .82

3134 RM 15.8 - DEAD OX WASH 15.82

3135 DEAD OX - RM 10.0

$3136 \mathrm{RM} 10.0$ - RM 9.2

13.18

3137 RM9.2 - NUMANA DAM

10.0

$9.7 \quad 1.9$

$.04 \quad .51$
.03

.14

47.

NO DIVERSIONS OR RETURNS

$N$ TRUCKEE DRAIN AT HEAD

STEAMBOAT C AT HEAD

NO DIVERSION

NOCE AND MURPHY DIVERSIONS

NOCE AND MURPHY RETURNS

L-NOCE \& L-MURPHY RETURNS

GROTON \& L-MURPHY RETURNS

L-MURPHY RETURNS

BACKWATER ABOVE MCCARRAN DAM

NO RETURNS

MCCARRAN RETURNS

BACKWATER ABOVE HILL DAM

NO RETURNS

NO RETURNS

HILL RETURNS

NO DIVERSIONS OR RETURNS

NO DIVERSIONS OR RETURNS

BACKWATER ABOVE DERBY DAM

TRUCKEE CANAL DIVERTS AT HEAD

NO DIVERSIONS OR RETURNS MODELED

WASHBURN-\& TRUCKEE CANAL RETURNS

NO RETURNS

GREGORY-MONTEAND CANAL RETURNS

HERMAN AND CANAL RETURNS

HERMAN AND CANAL RETURNS

HERMAN, PIERSON, \& CANAL RETURNS

FERNLEY AREA GROUND-WATER RETURNS

FERNLEY AREA GROUND-WATER RETURNS

OLINGHOUSE \#1 \& FELLNAGLE RETURNS

OLINGHOUSE \#1 \& FELLNAGLE RETURNS

$S$-S AND OLINGHOUSE \#2 RETURNS

S-S DAM, S-S PUMP \& OLINGH.\#3 RETURNS

SALINE GROUND WATER INFLOWS

SALINE GROUND WATER INFLOWS

SALINE GROUND WATER INFLOWS

9.20 SALINE GROUND WATER INFLOWS

3138 NUMANA DAM - RM 7.6

8.21

NO RETURNS

$3139 \mathrm{RM} 7.6$ - RM 6.8

7.60

NUMANA RETURNS

$3140 \mathrm{RM} 6.8$ - RM 4.0

6.80 NUMANA RETURNS

3141 RM 4.0 - NIXON BRIDGE 4.00

NUMANA RETURNS

3142 NIXON BRIDGE - RM 1.03 .22

NUMANA RETURNS

3143 RM 1.0 - MARBLE BLUFF D 1.00

NUMANA RETURNS, BACKWATER- ABOVE DAM

4101

4102

.085 .50

.0733 .50

.0728 .50

.0728 .50

.0778 .50

50. . . 1

54. . . 1

65. . . 1

63. . . 1

4104

67. .1 
APPENDIX II--LISTING OF EXAMPLE DATA SETS--Continued.

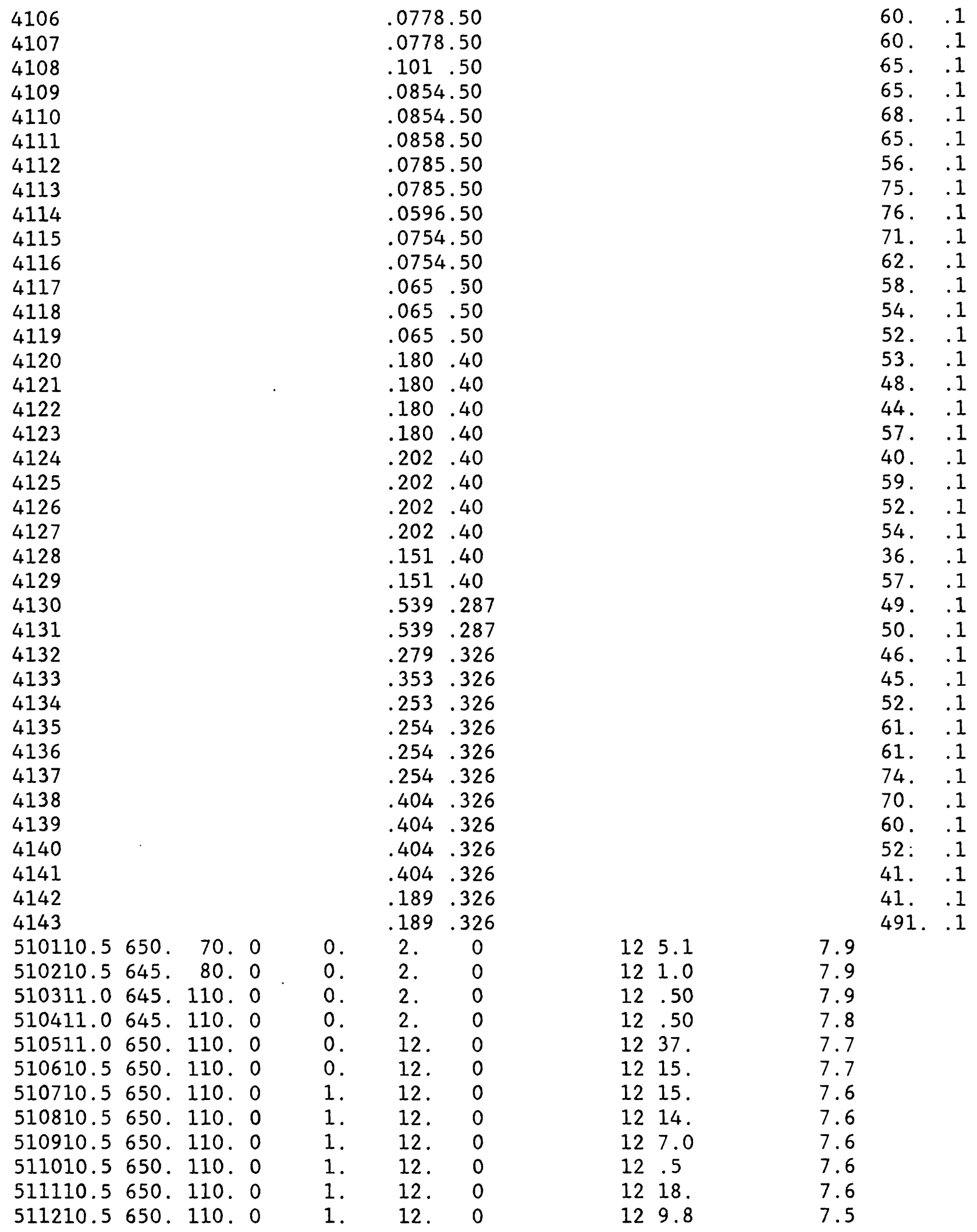


APPENDIX II--IISTING OF EXAMPLE DATA SETS--Continued.

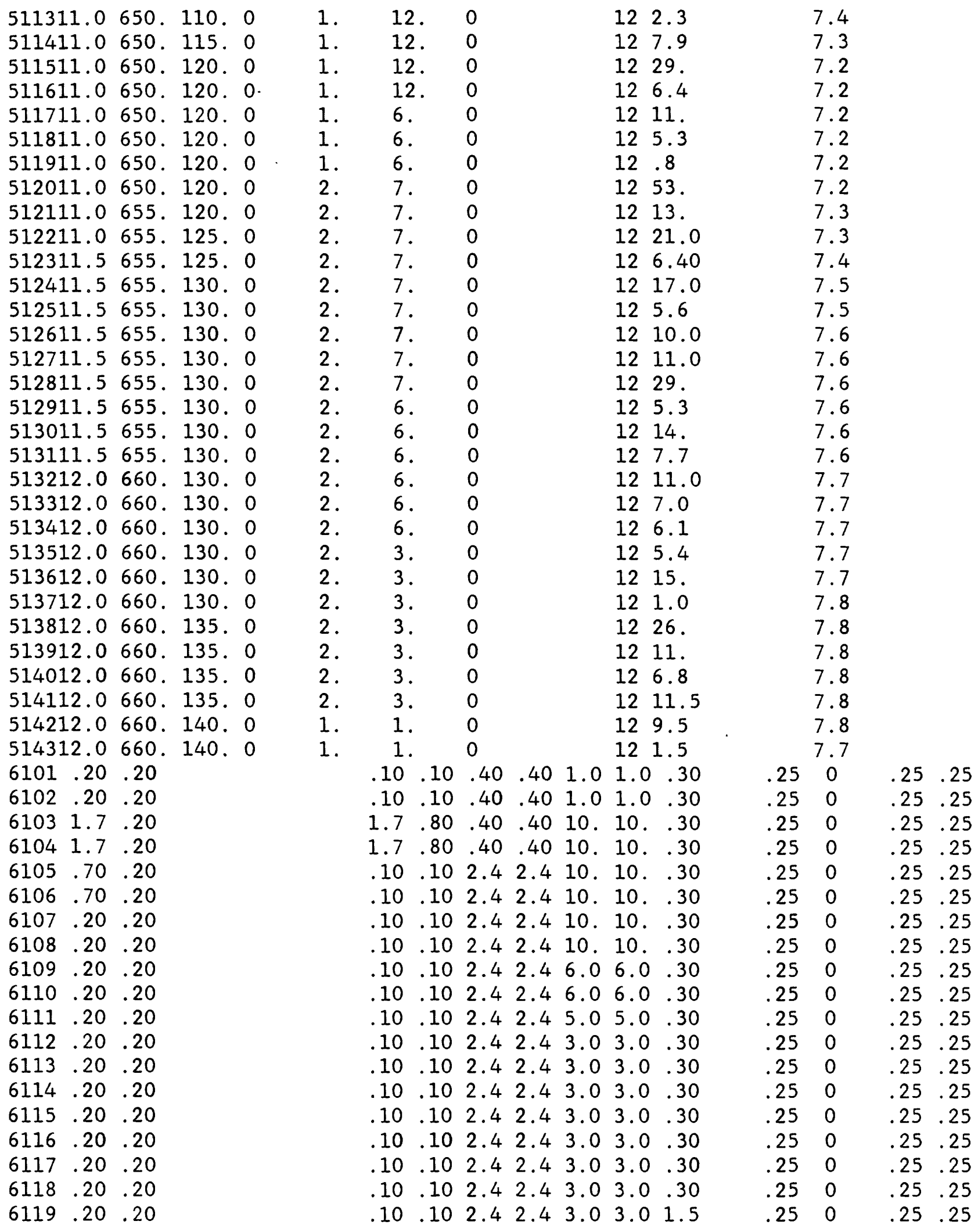


APPENDIX II--LISTING OF EXAMPLE DATA SETS--Continued.

$6120 \quad .20 \quad .20$

$6121.20 \quad .20$

6122.20 .20

$6123.20 \quad .20$

$6124 \quad .20 \quad .20$

$6125.20 \quad .20$

6126.20 .20

6127.20 .20

6128.20 .20

$6129.14 \quad .14$

$6130 \quad .14 \quad .14$

6131.14 .14

$6132 \quad .14 .14$

$6133 \quad .14 .14$

6134.14 .14

6135.14 .14

6136.14 .14

6137.14 .14

6138.14 .14

6139.14 .14

6140.14 .14

6141.14 .14

6142.14 .14

6143.14 .14

$7105-20$.

$7111-20.0$

$7115-4.0$

$7120-130$.

$7122-5.0$

$7124-10.0$

$7126-5.0$

$7127-5.0$

$7128-15.0$

$7130-10.0$

$7132-3.0$

$7138-16.0$

$\begin{array}{llllll}8106 & 1.2 & 22 . & 130 . & -.70 & 10.0\end{array}$

$\begin{array}{llllll}8107 & .4 & 22 . & 130 . & -.70 & 10.0\end{array}$

$\begin{array}{llllll}8108 & 4.3 & 22 . & 130 . & -.70 & 10.0\end{array}$

$\begin{array}{llllll}8109 & 4.1 & 22 . & 130 . & -.70 & 10.0\end{array}$

$\begin{array}{llllll}8111 & 1.0 & 22 . & 130 . & -.70 & 10.0\end{array}$

$\begin{array}{llllll}8112 & 9.0 & 22 . & 130 . & -.70 & 10.0\end{array}$

$\begin{array}{llllll}8120 & 3.5 & 22 . & 130 . & -.70 & 10.0\end{array}$

$812131.1 \quad 22 . \quad 130 . \quad-.70 \quad 10.0$

$\begin{array}{llllll}8122 & 15.0 & 22 . & 130 . & -.70 & 10.0\end{array}$

$\begin{array}{llllll}8123 & 6.0 & 22 . & 130 . & -.70 & 10.0\end{array}$

$\begin{array}{llllll}8124 & 15.5 & 22 . & 130 . & -.70 & 10.0\end{array}$

$812514.4 \quad 22 . \quad 130 . \quad-.70 \quad 10.0$

$\begin{array}{llllll}8126 & 7.8 & 22 . & 130 . & -.70 & 10.0\end{array}$

$\begin{array}{llllll}8127 & 24.1 & 22 . & 130 . & -.70 & 10.0\end{array}$
$102.42 .4 \quad 3.0 \quad 3.02 .0$

$\begin{array}{lllllll}10 & .10 & 2.4 & 2.4 & 3.0 & 3.0 & 2.0\end{array}$

.25

.25

.2

.25

.25

.25

.25

.25

.25

.25

.25

.25

.25

.25

.25

.25

.25

.2

.2

.25

.25

.25
$.25 \quad .25$

.25 .25

$.25 \quad .25$

$.25 \quad .25$

.25 .25

$.25 \quad .25$

$.25 \quad .25$

.25 .25

$.25 \quad .25$

$.25 \quad .25$

$.25 \quad .25$

$.25 \quad .25$

$.25 \quad .25$

$.25 \quad .25$

.25 .25

$.25 \quad .25$

$.25 \quad .25$

$.25 \quad .25$

$.25 \quad .25$

$.25 \quad .25$

$.25 \quad .25$

$.25 \quad .25$

$.25 \quad .25$

$.25 \quad .25$ $\begin{array}{lllllll}1.3 & .10 & .10 & .30 & .50 & -1.20 & 05\end{array}$

$\begin{array}{lllllll}1.3 & .10 & .10 & .30 & .50 & -1.20 & 05\end{array}$

$\begin{array}{lllllll}1.3 & .10 & .10 & .30 & .50 & -1.20 & 05\end{array}$

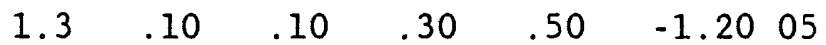

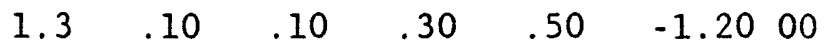

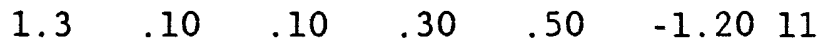

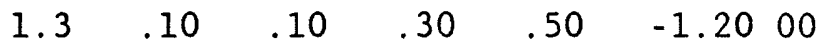

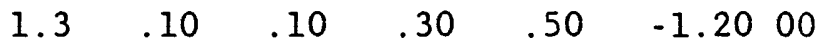

$\begin{array}{lllllll}1.3 & .10 & .10 & .30 & .50 & -1.20 & 00\end{array}$

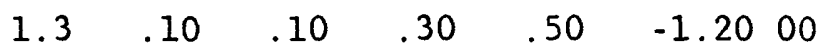

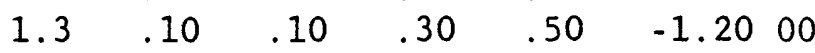

$\begin{array}{lllllll}1.3 & .10 & .10 & .30 & .50 & -1.20 & 00\end{array}$

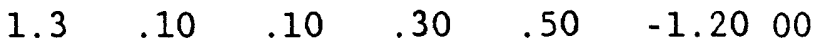

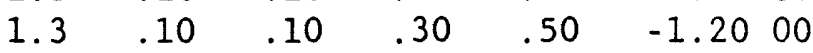


APPENDIX II--LISTING OF EXAMPLE DATA SETS--Continued.

\begin{tabular}{|c|c|c|c|c|c|c|c|c|c|c|c|c|}
\hline 8128 & 2.6 & 22 . & 130. & -.70 & 10.0 & 1.3 & .10 & .10 & .30 & .50 & -1.20 & 00 \\
\hline 8129 & 3.7 & 22. & 130. & -.70 & 10.0 & 1.3 & .10 & .10 & .30 & .50 & -1.20 & 00 \\
\hline 8130 & 4.2 & 22 . & 130. & -.70 & 10.0 & 1.3 & .10 & .10 & .30 & .50 & -1.20 & 00 \\
\hline 8131 & 10.6 & 22 . & 100. & -.70 & 10.0 & 1.3 & .10 & .10 & .30 & .50 & -1.20 & 00 \\
\hline 8132 & 10.3 & 22 . & 130. & -.70 & 10.0 & 1.3 & .10 & .10 & .30 & .50 & -1.20 & 00 \\
\hline 8133 & 10.6 & 22 . & 100. & -.70 & 10.0 & 1.3 & .10 & .10 & .30 & .50 & -1.20 & 00 \\
\hline 8134 & 8.5 & 22 . & 130. & -.70 & 10.0 & 1.3 & .10 & .10 & .30 & .50 & -1.20 & 00 \\
\hline 8135 & 10.4 & 22 . & 130. & -.70 & 10.0 & 1.3 & .10 & .10 & .30 & .50 & -1.20 & 00 \\
\hline 8136 & 2.6 & 22 . & 130. & -.70 & 10.0 & 1.3 & .10 & .10 & .30 & .50 & -1.20 & 00 \\
\hline 8137 & 0.0 & 22 . & 130. & -.70 & 10.0 & 1.3 & .10 & .10 & .30 & .50 & -1.20 & 00 \\
\hline 8138 & 0.0 & 22 . & 130. & -.70 & 10.0 & 1.3 & .10 & .10 & .30 & .50 & -1.20 & 00 \\
\hline 8139 & 0.0 & 22 . & 130. & -.70 & 10.0 & 1.3 & .10 & .10 & .30 & .50 & -1.20 & 00 \\
\hline 8140 & 11.3 & 22 . & 130. & -.70 & 10.0 & 1.3 & .10 & .10 & .30 & .50 & -1.20 & 38 \\
\hline 8141 & 3.2 & 22 . & 130. & -.70 & 10.0 & 1.3 & .10 & .10 & .30 & .50 & -1.20 & 38 \\
\hline 8142 & 0.0 & 22 . & 130. & -.70 & 10.0 & 1.3 & .10 & .10 & .30 & .50 & -1.20 & 00 \\
\hline 8143 & 0.0 & 22 . & 130. & -.70 & 10.0 & 1.3 & .10 & .10 & .30 & .50 & -1.20 & 00 \\
\hline 8206 & -1.20 & & .50 & .60 & & & & & & & & \\
\hline 8207 & -1.20 & & .50 & .60 & & & & & & & & \\
\hline 8208 & -1.20 & & .50 & .60 & & & & & & & & \\
\hline 8209 & -1.20 & & .50 & .60 & & & & & & & & \\
\hline 8210 & -1.20 & & .50 & .60 & & & & & & & & \\
\hline 8211 & -1.20 & & .50 & .60 & & & & & & & & \\
\hline 8212 & -1.20 & & .50 & .60 & & & & & & & & \\
\hline 8213 & -1.20 & & .50 & .60 & & & & & & & & \\
\hline 8214 & -1.20 & & .50 & .60 & & & & & & & & \\
\hline 8215 & -1.20 & & .50 & .60 & & & & & & & & \\
\hline 8216 & -1.20 & & .50 & .60 & & & & & & & & \\
\hline 8217 & -1.20 & & .50 & .60 & & & & & & & & \\
\hline 8218 & -1.20 & & .50 & .60 & & & & & & & & \\
\hline 8219 & -1.20 & & .50 & .60 & & & & & & & & \\
\hline 8220 & -1.20 & & .50 & .60 & & & & & & & & \\
\hline 8221 & -1.20 & & .50 & .60 & & & & & & - & & \\
\hline 8222 & -1.20 & & .50 & .60 & & & & & & & & \\
\hline 8223 & -1.20 & & .50 & .60 & & & & & & & & \\
\hline 8224 & -1.20 & & .50 & .60 & & & & & & & & \\
\hline 8225 & -1.20 & & .50 & .60 & & & & & & & & \\
\hline 8226 & -1.20 & & .50 & .60 & & & & & & & & \\
\hline 8227 & -1.20 & & .50 & .60 & & & & & & & & \\
\hline 8228 & -1.20 & & .50 & .60 & & & & & & & & \\
\hline 8229 & -1.20 & & .50 & .60 & & & & & & & & \\
\hline 8230 & -1.20 & & .50 & .60 & & & & & & & & \\
\hline 8231 & -1.20 & & .50 & .60 & & & & & & & & \\
\hline 8232 & -1.20 & & .50 & .60 & & & & & & & & \\
\hline 8233 & -1.20 & & .50 & .60 & & & & & & & & \\
\hline 8234 & -1.20 & & .50 & .60 & & & & & & & & \\
\hline 8235 & -1.20 & & .50 & .60 & & & & & & & & \\
\hline 8236 & -1.20 & & .50 & .60 & & & & & & & & \\
\hline 8237 & -1.20 & & .50 & .60 & & & & & & & & \\
\hline 8238 & -1.20 & & .50 & .60 & & & & & & & & \\
\hline 8239 & -1.20 & & .50 & .60 & & & & & & & & \\
\hline
\end{tabular}


APPENDIX II--LISTING OF EXAMPLE DATA SETS--Continued.

\begin{tabular}{|c|c|c|c|c|c|c|c|c|}
\hline 8240 & -1.20 & & .50 & .60 & & & & \\
\hline 8241 & -1.20 & & .50 & .60 & & & & \\
\hline 8242 & -1.20 & & .50 & .60 & & & & \\
\hline 8243 & -1.20 & & .50 & .60 & & & & \\
\hline 8307 & .5 & 17.0 & 1470 . & .5 & 1. & 1.9 & .1 & 1470 \\
\hline 8308 & 5.8 & 17.0 & 850 & .5 & 1. & 2.5 & .1 & 850 \\
\hline 8309 & 5.7 & 17.0 & 360. & .5 & 1. & 1.6 & .1 & 360 \\
\hline 8310 & 1.2 & 17.0 & 360. & .5 & 1. & 1.6 & .1 & 360 . \\
\hline 8311 & 5.1 & 17.0 & 360. & .5 & 1. & 1.6 & .1 & 360. \\
\hline 8312 & 7.2 & 17.0 & 360. & .5 & 1. & 1.6 & .1 & 360 \\
\hline 8313 & 3.1 & 17.0 & 360. & .5 & 1. & 1.6 & .1 & 360 . \\
\hline 8314 & 4.5 & 17.0 & 360. & .5 & 1. & 1.6 & .1 & 360. \\
\hline 8315 & .5 & 17.0 & 360 & .5 & 1. & 1.6 & .1 & 360 \\
\hline 8316 & 7.2 & 17.0 & 360. & .5 & 1. & 1.6 & .1 & 360. \\
\hline 8317 & 5.3 & 17.0 & 360 & .5 & 1. & 1.6 & .1 & 360 . \\
\hline 8318 & 5.3 & 17.0 & 360. & .5 & 1. & 1.6 & .1 & 360 . \\
\hline 8319 & 2.6 & 17.0 & 360. & .5 & 1. & 1.6 & .1 & 360. \\
\hline 8329 & 4.8 & 17.0 & 730. & .5 & 1. & 1.8 & .1 & 730 \\
\hline 8330 & 4.9 & 17.0 & 730. & .5 & 1. & 1.8 & .1 & 730. \\
\hline 8331 & .2 & 17.0 & 1080. & .5 & 1. & .5 & .1 & 1080 \\
\hline 8332 & .4 & 17.0 & 1080. & .5 & 1. & .5 & .1 & 1080 \\
\hline 8333 & .3 & 17.0 & 1080. & .5 & 1. & .5 & .1 & 1080 \\
\hline 8334 & .4 & 17.0 & 1080. & .5 & 1. & .5 & .1 & 1080 \\
\hline 8335 & .9 & 17.0 & 2250 . & .5 & 1. & .1 & .1 & 2250 \\
\hline 8336 & .2 & 17.0 & 2250 . & .5 & 1. & .1 & .1 & 2250 \\
\hline 8337 & .2 & 17.0 & 2250 . & .5 & 1. & .1 & .1 & 2250 \\
\hline 8338 & .2 & 17.0 & 780 & .5 & 1. & 1.0 & .1 & 780 \\
\hline 8339 & .2 & 17.0 & 780 & .5 & 1. & 1.0 & .1 & 780. \\
\hline 8340 & .7 & 17.0 & 780 & .5 & 1. & 1.0 & .1 & 780. \\
\hline 8341 & .2 & 17.0 & 780. & .5 & 1. & 1.0 & .1 & 780 \\
\hline 8342 & .7 & 17.0 & 780 & .5 & 1. & 1.0 & .1 & 780 \\
\hline 8343 & .3 & 17.0 & 2410 . & .5 & 1. & 1.0 & .1 & 2410 \\
\hline 8407 & 1090. & & .1 & .1 & & & & \\
\hline 8408 & $630 \ldots$ & & .1 & .1 & & & & \\
\hline 8409 & 270 & & .1 & .1 & & & & \\
\hline 8410 & 270 & & .1 & .1 & & & & \\
\hline 8411 & 270 & & .1 & .1 & & & & \\
\hline 8412 & 270 . & & .1 & .1 & & & & \\
\hline 8413 & 270 & & .1 & .1 & & & & \\
\hline 8414 & 270 & & .1 & .1 & & & & \\
\hline 8415 & 270 & & .1 & .1 & & & & \\
\hline 8416 & 270 & & .1 & .1 & & & & \\
\hline 8417 & 270 & & .1 & .1 & & & & \\
\hline 8418 & 270 & & .1 & .1 & & & & \\
\hline 8419 & 270 & & .1 & .1 & & & & \\
\hline 8429 & 540 . & & .1 & .1 & & & & \\
\hline 8430 & 540. & & .1 & .1 & & & & \\
\hline 8431 & 800 . & & .1 & .1 & & & & \\
\hline 8432 & 800 . & & .1 & .1 & & & & \\
\hline 8433 & 800 . & & .1 & .1 & & & & \\
\hline
\end{tabular}


APPENDIX II--LISTING OF EXAMPLE DATA SETS--Continued.

\begin{tabular}{|c|c|c|c|c|c|c|c|c|c|c|}
\hline 843480 & & .1 & .1 & & & & & & & \\
\hline 843516 & & .1 & .1 & & & & & & & \\
\hline 843616 & & .1 & .1 & & & & & & & \\
\hline 843716 & & .1 & .1 & & & & & & & \\
\hline 843858 & & .1 & .1 & & & & & & & \\
\hline 843958 & & .1 & .1 & & & & & & & \\
\hline 844058 & & .1 & .1 & & & & & & & \\
\hline 844158 & & .1 & .1 & & & & & & & \\
\hline 844258 & & .1 & .1 & & & & & & & \\
\hline 844317 & & .1 & .1 & & & & & & & \\
\hline 9156.12 & 10.4 & 107. & 2.9 & & .69 & .17 & .01 & .36 & .08 & 72. \\
\hline 9156.12 & 9.7 & 101. & 1.9 & & .51 & .14 & .00 & .24 & .04 & 70. \\
\hline 9156.12 & 9.2 & 93. & 1.4 & & .24 & .12 & .00 & .11 & .01 & 64. \\
\hline 9152.23 & 9.8 & 108. & 2.7 & & .78 & .42 & .02 & .33 & .15 & 123. \\
\hline 9152.23 & 9.5 & 101. & 2.5 & & .40 & .31 & .01 & .24 & .12 & 115. \\
\hline 9152.23 & 8.9 & 95. & 2.0 & & .18 & .22 & .00 & .10 & .09 & 106. \\
\hline 9150.05 & 9.8 & 103. & 3.0 & & .44 & .39 & .01 & .26 & .14 & 121. \\
\hline 9150.05 & 9.3 & 97. & 2.4 & & .42 & .33 & .01 & .20 & .13 & 112. \\
\hline 9150.05 & 8.6 & 92. & 2.0 & & .36 & .26 & .01 & .13 & .11 & 102. \\
\hline 9144.92 & 9.7 & 103. & 3.1 & & .75 & .36 & .02 & .45 & .14 & 119. \\
\hline 9144.92 & 9.2 & 96. & 2.6 & & .60 & .26 & .01 & .21 & .11 & 112. \\
\hline 9144.92 & 8.7 & 93. & 2.4 & & .40 & .15 & .01 & .10 & .08 & 105. \\
\hline 9140.62 & 9.9 & 106. & 5.0 & & .88 & .32 & .02 & .47 & .15 & 125. \\
\hline 9140.62 & 9.1 & 96. & 3.0 & & .63 & .26 & .02 & .23 & .12 & 121. \\
\hline 9140.62 & 8.7 & 91. & 2.2 & & .08 & .14 & .01 & .11 & .04 & 117. \\
\hline 9134.88 & 9.4 & 103. & 3.6 & & .90 & .34 & .03 & .60 & .14 & 126. \\
\hline 9134.88 & 9.0 & 95. & 2.8 & & .64 & .26 & .02 & .28 & .10 & 121. \\
\hline 9134.88 & 8.6 & 89. & 2.4 & & .46 & .22 & .01 & .12 & .06 & 115. \\
\hline 9134.52 & 9.7 & 107. & & & & & & & & 131. \\
\hline 9134.52 & 9.2 & 98. & & & & & & & & 124. \\
\hline 9134.52 & 9.0 & 93. & & & & & & & & 127. \\
\hline 9129.97 & 9.5 & 102. & 3.2 & & .63 & .23 & .03 & .48 & .15 & 134. \\
\hline 9129.97 & 9.2 & 98. & 2.7 & & .48 & .20 & .02 & .30 & .13 & 128. \\
\hline 9129.97 & 8.9 & 93. & 2.1 & & .36 & .13 & .01 & .18 & .12 & 122. \\
\hline 9123.65 & 9.6 & 101. & 2.8 & & .96 & .26 & .04 & .43 & .21 & 148. \\
\hline 9123.65 & 9.3 & 98. & 2.5 & & .62 & .20 & .03 & .27 & .13 & 127. \\
\hline 9123.65 & 9.2 & 95. & 2.1 & & .34 & .13 & .01 & .18 & .09 & 111. \\
\hline 9113.18 & 9.4 & 103. & 3.8 & & .60 & .18 & .03 & .52 & .15 & 139. \\
\hline 9113.18 & 9.1 & 97. & 2.7 & & .40 & .13 & .02 & .30 & .12 & 129. \\
\hline 9113.18 & 8.9 & 92. & 2.0 & & .20 & .10 & .01 & .20 & .09 & 118. \\
\hline 913.22 & 9.7 & 102. & 3.6 & & .72 & .13 & .03 & .31 & .15 & 145. \\
\hline 913.22 & 9.3 & 99. & 2.9 & & .45 & .08 & .02 & .25 & .11 & 141. \\
\hline 913.22 & 8.9 & 95. & 2.1 & & .30 & .04 & .02 & .16 & .06 & 135. \\
\hline 910. & 9.4 & 102. & 3.0 & & .51 & .29 & .03 & .87 & .15 & 149. \\
\hline 910. & 9.1 & 97. & 2.6 & & .32 & .21 & .03 & .43 & .12 & 144. \\
\hline 910. & 8.6 & 91. & 2.2 & & .24 & .10 & .02 & .24 & .09 & 137. \\
\hline 9249. & & .08 & .07 & 1950. & .003 & 1.43 & 413. & & & \\
\hline 9247. & & .04 & .03 & 1790. & .002 & 1.43 & 413. & & & \\
\hline 9243. & & .01 & .01 & 1740 . & .001 & 1.43 & 413. & & & \\
\hline 9285. & & .16 & .12 & 2130 . & .004 & .57 & 483. & & & \\
\hline
\end{tabular}


APPENDIX II--LISTING OF EXAMPLE DATA SETS--Continued.

\begin{tabular}{|c|c|c|c|c|c|c|}
\hline 9281. & .12 & .10 & 2010. & .003 & .57 & 470. \\
\hline 9276. & .09 & .07 & 1960. & .002 & .57 & 458. \\
\hline 9284. & .14 & .17 & 2060 . & .003 & 1.28 & 596 \\
\hline 9279. & .13 & .12 & 1990. & .002 & 1.28 & 476 \\
\hline 9274 . & .11 & .09 & 1940. & .001 & 1.28 & 355 \\
\hline 9283. & .14 & .13 & 2060 . & .003 & & 512 \\
\hline 9279. & .11 & .11 & 1990. & .002 & & 512 \\
\hline 9275 . & .08 & .07 & 1940. & .001 & & 512 \\
\hline 9287. & .15 & .13 & 2060 . & .001 & & 665 \\
\hline 9284. & .12 & .11 & 2015. & .001 & & 539 \\
\hline 9282. & .04 & .08 & 1940. & 0.00 & & 412 \\
\hline 9287. & .14 & .12 & 2110 . & .000 & 1.5 & 526 \\
\hline 9284. & .10 & .11 & 2040. & .001 & .80 & 502 \\
\hline 9281. & .06 & .08 & 1990. & .000 & .11 & 478 \\
\hline 9290. & & & 1950. & 0.00 & & \\
\hline 9286. & & . & 1910. & 0.00 & & \\
\hline 9282 . & & & 1860. & 0.00 & & \\
\hline 9292. & .15 & .14 & 1960. & .002 & 1.50 & 516 \\
\hline 9288. & .13 & .12 & 1940. & .002 & 1.02 & 44 \\
\hline 9285. & .12 & .10 & 1870. & .001 & .58 & 382 \\
\hline 92100. & .21 & .21 & 2060 . & .003 & 1.47 & 666 \\
\hline 9288. & .13 & .13 & 1990. & .002 & 1.13 & 564 \\
\hline 9279. & .09 & .09 & 1940. & .001 & .93 & 462 \\
\hline 9295. & .15 & .13 & 2090. & .003 & & 42 \\
\hline 9289. & .12 & .11 & 2040 . & .002 & & 38 \\
\hline 9283. & .09 & .09 & 1980. & .001 & & 348 \\
\hline 9298. & .15 & .15 & 2150 . & .003 & 1.23 & 442 \\
\hline 9296 . & .11 & .11 & 2050 . & .001 & 1.23 & 40 \\
\hline 9292. & .06 & .09 & 1990. & .000 & 1.23 & 36 \\
\hline 92100. & .15 & .14 & 2100 . & .003 & .77 & 33. \\
\hline 9297. & .12 & .11 & 2060 . & .002 & .73 & 32 \\
\hline 9293. & .09 & .09 & 1990. & .001 & .69 & 31 \\
\hline
\end{tabular}


APPENDIX II--LISTING OF EXAMPLE DATA SETS--Continued.

D. - - Truckee River, August 1980

The following data set represents Truckee River conditions observed during field investigations in August 1980.

\begin{tabular}{|c|c|c|c|c|c|c|c|c|c|c|}
\hline \multicolumn{11}{|c|}{ C R880.PUB } \\
\hline \multicolumn{11}{|c|}{ A1 010} \\
\hline \\
\hline \multirow{2}{*}{\multicolumn{11}{|c|}{ 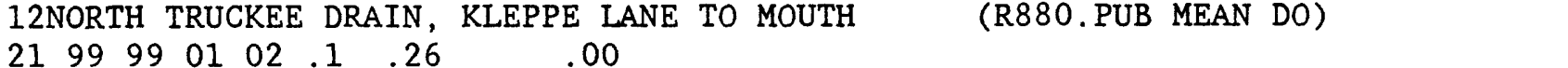 }} \\
\hline & & & & & & & & & & \\
\hline 2240.0 & 17.5 & & & 348 . & .0 & 4.0 & & 1.0 & .0 & \\
\hline 23.02 & .44 & & & 348 . & 42. & & .04 & .11 & & \\
\hline $3101 \mathrm{KLE}$ & PPE LAN & NE TO & UTH & .26 & NO DI & VERSIONS & $S$ OR RETL & & & \\
\hline 4101 & & & & .58 & 26 & & & & & 5.0 \\
\hline 510117.5 & 645.3 & 350. & & & & & 122.3 & & 7.8 & \\
\hline 6101.20 & .20 & & & .10 .10 & .40 & .401 .0 & 1.0 .30 & .25 & 0 & $.25 \quad .25$ \\
\hline 91.26 & 10.8 & 143. & 4.4 & & 1.4 & .07 & .04 & .51 & .06 & 384 \\
\hline 91.26 & 8.0 & 99. & 4.0 & & 1.0 & .04 & .02 & .44 & .04 & 348 . \\
\hline 91.26 & 5.6 & 66. & 3.7 & & .51 & .01 & .01 & .35 & .00 & 299. \\
\hline 92267. & & .06 & .15 & 41. & .006 & 1.0 & 1850. & & & \\
\hline 92242 . & & .04 & .11 & 40. & .001 & .94 & 1650. & & & \\
\hline 92208. & & .00 & .09 & 33. & .000 & .87 & 1380. & & & \\
\hline
\end{tabular}

11TRUCKEE RIVER, MCCARRAN BRIDGE TO DERBY DAM

12STEMBOAT CREEK, KIMLICK LANE TO MOUTH (R880.PUB MEAN DO)

$\begin{array}{llllllll}21 & 99 & 99 & 02 & 03 & .1 & .75 & .00\end{array}$

$\begin{array}{lllllllll}22 & 70.0 & 19.6 & 646 . & 290 . & 6.9 & 5.8 & 1.8 & .06\end{array}$

$\begin{array}{lllllll}23 & .02 & .07 & .09 & 290 . & 202 . & .09\end{array}$

3101 KIMLICK LANE TO STP .75 NO DIVERSIONS OR RETURNS

3102 STP OUTFALL TO MOUTH .13 STP OUTFALL ENTERS AT HEAD

4101

$.26 \quad .32$

$.088 \quad .30$

510119.5645 .290 .0

0

510221.0645 .380 .0

$\begin{array}{lllll}0 & 0 & 0 & 12.5\end{array}$

8.0

6101.20 .20

$\begin{array}{lllllllllllll}.10 & .10 & .40 & .40 & 1.0 & 1.0 & .30 & .25 & 0 & .25 & .25\end{array}$

$6102 \quad 1.7 .20$

$\begin{array}{llllllllllll}1.7 & .80 & .40 & .40 & 10 . & 10 . & .30 & .25 & 0 & .25 & .25\end{array}$

$\begin{array}{lllllll}7102 & 35.0 & 23.3 & 646 . & 572 . & 7.6 & 39 .\end{array}$

6.14.

14. .20

14. .20

$7202572.327 . \quad 3.54 .4$

$\begin{array}{llllllllll}91.75 & 9.0 & 126 . & 6.3 & 2.9 & .09 & .03 & .09 & .13 & 302 .\end{array}$

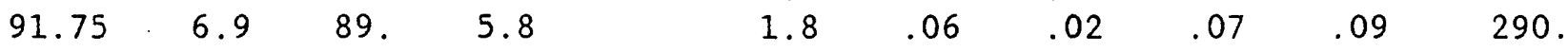

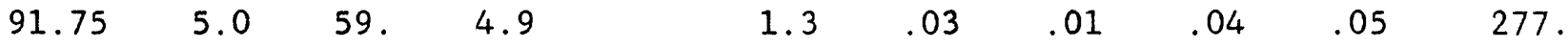

$\begin{array}{lllllll}92210 . & .13 & .21 & 79 . & .014 & 2.6 & 2060 .\end{array}$

92202. $\quad .09 \quad .16 \quad 70 . \quad .003 \quad 1.8 \quad 1750$.

92193. $\quad .05 \quad .14 \quad 64 . \quad .001 \quad .89 \quad 1160$.

11TRUCKEE RIVER, MCCARRAN BRIDGE TO DERBY DAM

12MAINSTEM TRUCKEE RIVER

$\begin{array}{lllllllll}21 & 99 & 99 & 43 & 00 & .10 & 56.12 & 0.00 & 0\end{array}$

(R880. PUB MEAN DO) 
APPENDIX II--LISTING OF EXAMPLE DATA SETS--Continued.

$\begin{array}{llllllllll}22 & 155.0 & 17.9 & 646 . & 126 . & 8.3 & 2.5 & & .52 & .03 \\ 23 & .02 & .00 & .02 & 126 . & 85 . & & .02 & .07 & \end{array}$

3101 MCCARRAN BR - N TRUCKEE 56.12 NO DIVERSIONS OR RETURNS

$3102 \mathrm{~N}$ TRUCKEE DR - STEAMBT 53.66 N TRUCKEE DRAIN AT HEAD

3103 STEAMBOAT - VISTA 53.53 STEAMBOAT C AT HEAD

3104 VISTA - LARGOMARSINO DV 52.23 NO DIVERSION

3105 LARGOMARSINO DIVERSIONS 51.25

3106 BELOW LARG. - LOCKWOOD 50.90

3107 LOCKWOOD - GROTON DIV 50.05

3108 GROTON DV - MUSTANG BR\#149.90

3109 MUSTANG BR - LAST RET. 48.25

3110 MCCARRAN POOL 46.68

3111 MCCARRAN DV - PATRICK B 46.35

3112 PATRICK BR - SP RR BR 44.92

3113 SP RR BR - HILL DIV 42.88

3114 HILL DIV - TRACY DIV 42.02

3115 TRACY DIV - TRACY BRIDGE40.76

3116 TRACY BR - CLARK BR 40.62

3117 CLARK BR - RM $37.1 \quad 38.60$

3118 RM 37.1 - I-80 OXBOW 37.10

3119 I-80 OXBOW - DERBY DAM 35.60

3120 DERBY DAM - GAGE CABLE 34.88

3121 GAGE CABLEWAY - WASHBRN 34.52

3122 WASHBURN - PAINTED ROCK 31.28

3123 PAINTED R - GREGORY-MONT29.97

3124 GREGORY-MONTE - RM 28.029 .35

3125 RM 28.0 - HERMAN DIV 28.00

3126 HERMAN DIV - PIERSON DIV26.75

3127 PIERSON DIV - PROCTR DIV25.95

3128 PROCTOR - WADSWORTH 23.90

3129 WADSWORTH -FELLNAGLE DN 23.69

3130 FELLNAGLE - RM $21.4 \quad 22.55$

3131 RM 21.4 - S-BAR-S DIV 21.40

3132 S-S DAM - S-S PUMP 19.84

$\begin{array}{llll}3133 & \text { S-BAR-S PUMP - RM } 15.8 & 17.82\end{array}$

3134 RM 15.8 - DEAD OX WASH 15.82

3135 DEAD OX - RM $10.0 \quad 13.18$

3136 RM 10.0 - RM $9.2 \quad 10.0$

3137 RM9.2 - NUMANA DAM 9.20

3138 NUMANA DAM - RM $7.6 \quad 8.21$

3139 RM 7.6 - RM 6.8 7.60

$3140 \mathrm{RM} 6.8$ - RM $4.0 \quad 6.80$

3141 RM 4.0 - NIXON BRIDGE 4.00

3142 NIXON BRIDGE - RM 1.03 .22

NOCE AND MURPHY DIVERSIONS

NOCE AND MURPHY RETURNS

L-NOCE \& L-MURPHY RETURNS

GROTON \& L-MURPHY RETURNS

L-MURPHY RETURNS

BACKWATER ABOVE MCCARRAN DAM

NO RETURNS

MCCARRAN RETURNS

BACKWATER ABOVE HILL DAM

NO RETURNS

NO RETURNS

HILL RETURNS

NO DIVERSIONS OR RETURNS

NO DIVERSIONS OR RETURNS

BACKWATER ABOVE DERBY DAM

TRUCKEE CANAL DIVERTS AT HEAD

NO DIVERSIONS OR RETURNS MODELED

WASHBURN \& TRUCKEE CANAL RETURNS

NO RETURNS

GREGORY-MONTEAND CANAL RETURNS

HERMAN AND CANAL RETURNS

HERMAN AND CANAL RETURNS

HERMAN, PIERSON , \& CANAL RETURNS

FERNLEY AREA GROUND-WATER RETURNS

FERNLEY AREA GROUND-WATER RETURNS

OLINGHOUSE \#1 \& FELLNAGLE RETURNS

OLINGHOUSE \#1 \& FELLNAGLE RETURNS

$S-S$ AND OLINGHOUSE \#2 RETURNS

S-S DAM, S-S PUMP \& OLINGH.\#3 RETURNS

SALINE GROUND WATER INFLOWS

SALINE GROUND WATER INFLOWS

SALINE GROUND WATER INFLOWS

SALINE GROUND WATER INFLOWS

NO RETURNS

NUMANA RETURNS

NUMANA RETURNS

NUMANA RETURNS

NUMANA RETURNS

3143 RM 1.0 - MARBLE BLUFF D 1.00

NUMANA RETURNS, BACKWATER ABOVE DAM

4101

.0695 .50

.0733 .50

.0351 .603

4103

.0351 .603

.0137 .765

50. .1

54. . 1

65. . 1

63. . 1

4105

$67 . .1$ 
APPENDIX II--LISTING OF EXAMPLE DATA SETS--Continued.

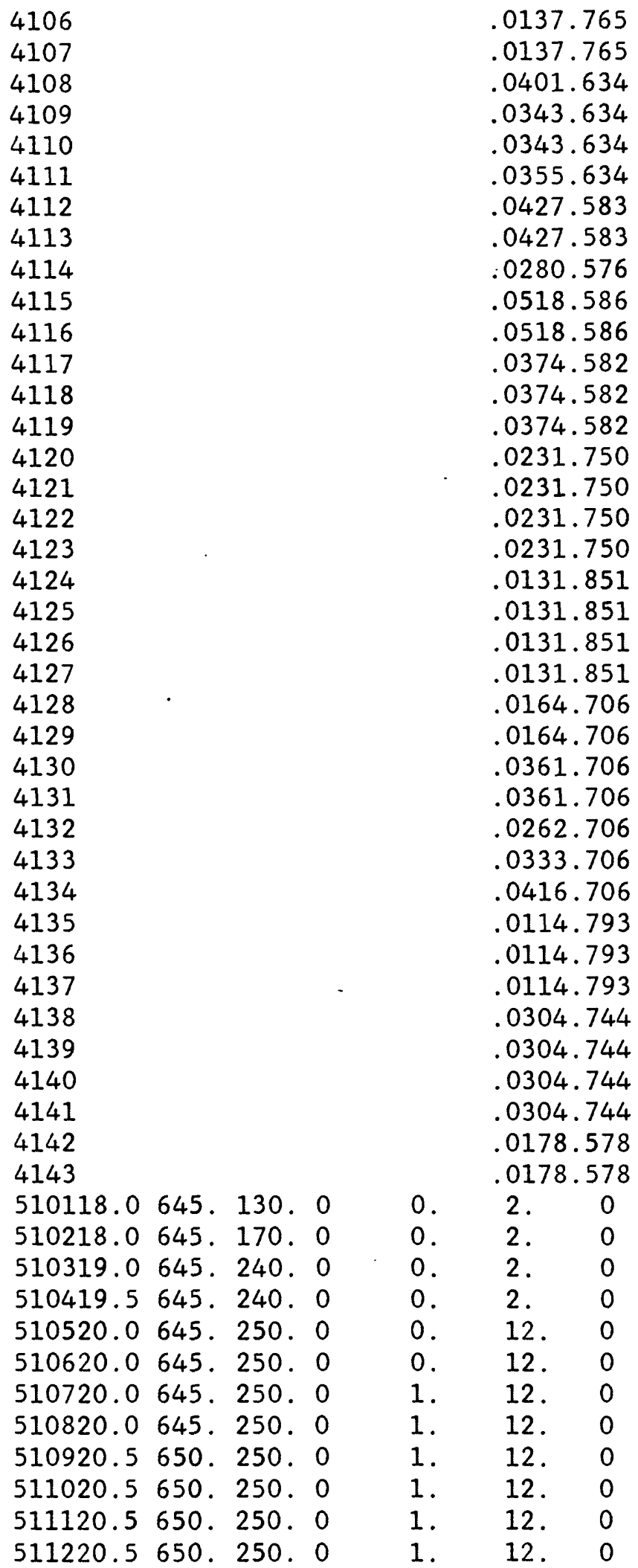

60. .1

60. .1

65. .1

55. . 1

68. .1

65. . 1

56. . 1

75. .1

76. .1

71. 1

62. . 1

58. . 1

54. . 1

52. . 1

53. . 1

48. . 1

44. . 1

57. . 1

40. .1

59. . 1

52. .1

54. . 1

36 . .1

57. . 1

49. . 1

50. . 1

46. .1

45. . 1

52. .1

61. . 1

61. . 1

74. .1

70. .1

60 . .1

52. . 1

41. . 1

41. . 1

491. . 1

$\begin{array}{lll}12 & 5.1 & 8.1\end{array}$

$121.0 \quad 8.2$

$12.50 \quad 8.1$

$12.50 \quad 8.0$

$1237 . \quad 8.0$

$1215 . \quad 7.9$

$1215 . \quad 7.9$

$1214 . \quad 7.9$

$\begin{array}{lll}12 & 7.0 & 7.8\end{array}$

$\begin{array}{lll}12.5 & 7.7\end{array}$

$1218 . \quad 7.7$

$\begin{array}{lll}12 & 9.8 & 7.7\end{array}$ 
APPENDIX II--LISTING OF EXAMPLE DATA SETS--Continued.

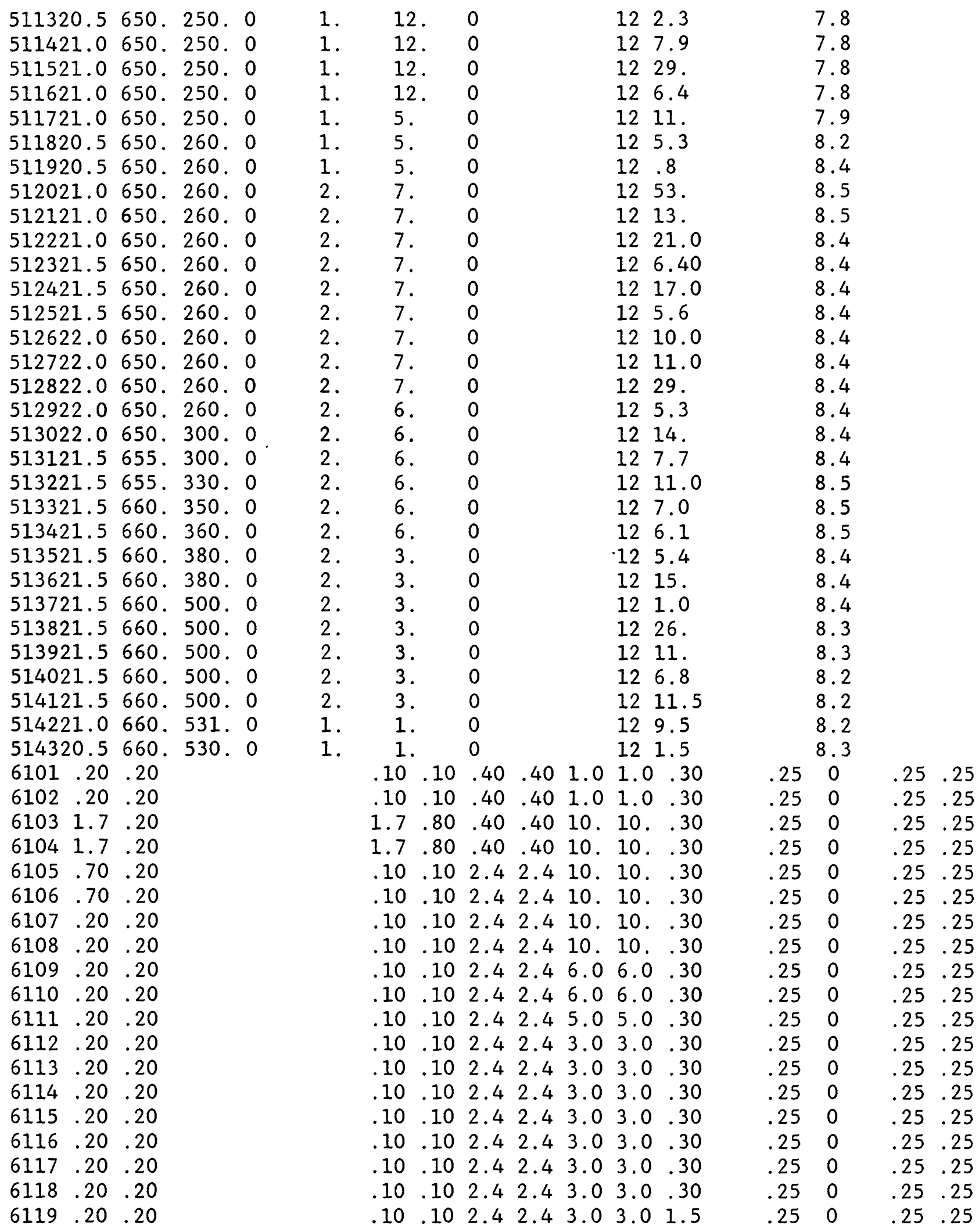


APPENDIX II--LISTING OF EXAMPLE DATA SETS--Continued.

\begin{tabular}{|c|c|c|c|c|c|c|c|c|c|c|c|c|c|c|c|}
\hline 6120 & .20 & 20 & & & .10 & .10 & 2.4 & 2.4 & 3.0 & 3.0 & 2.0 & .25 & 0 & .25 & 25 \\
\hline 6121 & .20 & 20 & & & .10 & .10 & 2.4 & 2.4 & 3.0 & 3.0 & 2.0 & .25 & 0 & .25 & 25 \\
\hline 6122 & .20 & 20 & & & .10 & .10 & 2.4 & 2.4 & 3.0 & 3.0 & 2.0 & .25 & 0 & .25 & 25 \\
\hline 6123 & .20 & 20 & & & .10 & .10 & 2.4 & 2.4 & 3.0 & 3.0 & 2.0 & .25 & 0 & .25 & 25 \\
\hline 6124 & .20 & 20 & & & .10 & .10 & 2.4 & 2.4 & 3.0 & 3.0 & 2.0 & .25 & 0 & .25 & 25 \\
\hline 6125 & .20 & 20 & & & .10 & .10 & 2.4 & 2.4 & 3.0 & 3.0 & 2.0 & .25 & 0 & .25 & 25 \\
\hline 6126 & .20 & 20 & & & .10 & .10 & 2.4 & 2.4 & 3.0 & 3.0 & 2.0 & .25 & 0 & .25 & 25 \\
\hline 6127 & .20 & 20 & & & .10 & .10 & 2.4 & 2.4 & 3.0 & 3.0 & 2.0 & .25 & 0 & .25 & 25 \\
\hline 6128 & .20 & 20 & & & .10 & .10 & 2.4 & 2.4 & 3.0 & 3.0 & 2.0 & .25 & 0 & .25 & 25 \\
\hline 6129 & .14 & 14 & & & .10 & .10 & 2.4 & 2.4 & 3.0 & 3.0 & 2.0 & .25 & 0 & .25 & 25 \\
\hline 6130 & .14 & 14 & & & .10 & .10 & 2.4 & 2.4 & 3.0 & 3.0 & 2.0 & .25 & 0 & .25 & 25 \\
\hline 6131 & .14 & 14 & & & .10 & .10 & 2.4 & 2.4 & 3.0 & 3.0 & 2.0 & .25 & 0 & .25 & 25 \\
\hline 6132 & .14 & 14 & & & .10 & .10 & 2.4 & 2.4 & 3.0 & 3.0 & 2.0 & .25 & 0 & .25 & 25 \\
\hline 6133 & .14 & 14 & & & .10 & .10 & 2.4 & 2.4 & 3.0 & 3.0 & 2.0 & .25 & 0 & .25 & 25 \\
\hline 6134 & .14 & 14 & & & .10 & .10 & 2.4 & 2.4 & 3.0 & 3.0 & 2.0 & .25 & 0 & .25 & 25 \\
\hline 6135 & .14 & 14 & & & .10 & .10 & 2.4 & 2.4 & 3.0 & 3.0 & 2.0 & .25 & 0 & .25 & 25 \\
\hline 6136 & .14 & 14 & & & .10 & .10 & 2.4 & 2.4 & 3.0 & 3.0 & 2.0 & .25 & 0 & .25 & 25 \\
\hline 6137 & .14 & 14 & & & .10 & .10 & 2.4 & 2.4 & 3.0 & 3.0 & 2.0 & .25 & 0 & .25 & 25 \\
\hline 6138 & .14 & 14 & & & .10 & .10 & 2.4 & 2.4 & 3.0 & 3.0 & 2.0 & .25 & 0 & .25 & 25 \\
\hline 6139 & .14 & 14 & & & .10 & .10 & 2.4 & 2.4 & 3.0 & 3.0 & 2.0 & .25 & 0 & .25 & 25 \\
\hline 6140 & .14 & 14 & & & .10 & .10 & 2.4 & 2.4 & 3.0 & 3.0 & 2.0 & .25 & 0 & .25 & 25 \\
\hline 6141 & .14 & 14 & & & .10 & .10 & 2.4 & 2.4 & 3.0 & 3.0 & 2.0 & .25 & 0 & .25 & 25 \\
\hline 6142 & .14 & 14 & & & .10 & .10 & 2.4 & 2.4 & 3.0 & 3.0 & 2.0 & .25 & 0 & .25 & 25 \\
\hline 6143 & .14 & 14 & & & .10 & .10 & 2.4 & 2.4 & 3.0 & 3.0 & 2.0 & .25 & 0 & .25 & 25 \\
\hline 7105 & -19. & & & & & & & & & & & & & & \\
\hline 7108 & -3.0 & & & & & & & & & & & & & & \\
\hline 7111 & -10.0 & & & & & & & & & & & & & & \\
\hline 7114 & -7.0 & & & & & & & & & & & & & & \\
\hline 7115 & -4.0 & & & & & & & & & & & & & & \\
\hline 7120 & -205. & & & & & & & & & & & & & & \\
\hline 7122 & -1.0 & & & & & & & & & & & & & & \\
\hline 7124 & -5.0 & & & & & & & & & & & & & & \\
\hline 7126 & -15.0 & & & & & & & & & & & & & & \\
\hline 7127 & -6.0 & & & & & & & & & & & & & & \\
\hline 7128 & -6.0 & & & & & & & & & & & & & & \\
\hline 7130 & -11.0 & & & & & & & & & & & & & & \\
\hline 7132 & -4.0 & & & & & & & & & & & & & & \\
\hline 7138 & -20.0 & & & & & & & & & & & & & & \\
\hline 8106 & .8 & 22 . & 130. & -.70 & 10.0 & & & 1.3 & .1 & 0 & .10 & .30 & .50 & -1.20 & 05 \\
\hline 8107 & .2 & 22 . & 130. & -.70 & 10.0 & & & 1.3 & .1 & 0 & .10 & .30 & .50 & -1.20 & 05 \\
\hline 8108 & 3.8 & 22 . & 130. & -.70 & 10.0 & & & 1.3 & .1 & .0 & .10 & .30 & .50 & -1.20 & 05 \\
\hline 8109 & 2.6 & 22 . & 130. & -.70 & 10.0 & & & 1.3 & .1 & 0 & .10 & .30 & .50 & -1.20 & 05 \\
\hline 8111 & .3 & 22 . & 130. & -.70 & 10.0 & & & 1.3 & .1 & 0 & .10 & .30 & .50 & -1.20 & 00 \\
\hline 8112 & 3.0 & 22 . & 130. & -.70 & 10.0 & & & 1.3 & .1 & .0 & .10 & .30 & .50 & -1.20 & 11 \\
\hline 8114 & .3 & 22. & 130. & -.70 & 10.0 & & & 1.3 & .1 & 0 & .10 & .30 & .50 & -1.20 & 00 \\
\hline 8115 & .1 & 22 . & 130. & -.70 & 10.0 & & & 1.3 & .1 & .0 & .10 & .30 & .50 & -1.20 & 14 \\
\hline 8116 & 1.9 & 22. & 130. & -.70 & 10.0 & & & 1.3 & .1 & 0 & .10 & .30 & .50 & -1.20 & 14 \\
\hline 8120 & .4 & 22 . & 130. & -.70 & 10.0 & & & 1.3 & .1 & .0 & .10 & .30 & .50 & -1.20 & 00 \\
\hline 8121 & 3.6 & 22. & 130. & -.70 & 10.0 & & & 1.3 & .1 & 0 & .10 & .30 & .50 & -1.20 & 00 \\
\hline 8122 & 1.8 & 22 . & 130. & -.70 & 10.0 & & & 1.3 & .1 & 0 & .10 & .30 & .50 & -1.20 & 00 \\
\hline
\end{tabular}


APPENDIX II--LISTING OF EXAMPLE DATA SETS--Continued.

\begin{tabular}{|c|c|c|c|c|c|c|c|c|c|c|c|}
\hline 8123 & 0.7 & 22. & 130. & -.70 & 10.0 & 1.3 & .10 & .10 & .30 & .50 & -1.20 \\
\hline 8124 & 2.4 & 22. & 130. & -.70 & 10.0 & 1.3 & .10 & .10 & .30 & .50 & -1.20 \\
\hline 8125 & 2.3 & 22. & 130. & -.70 & 10.0 & 1.3 & .10 & .10 & .30 & .50 & -1.20 \\
\hline 8126 & 1.1 & 22. & 130. & -.70 & 10.0 & 1.3 & .10 & .10 & .30 & .50 & -1.20 \\
\hline 8127 & 9.1 & 22. & 130. & -.70 & 10.0 & 1.3 & .10 & .10 & .30 & .50 & -1.20 \\
\hline 8128 & 1.6 & 22. & 130. & -.70 & 10.0 & 1.3 & .10 & .10 & .30 & .50 & -1.20 \\
\hline 8130 & .2 & 22 . & 130 & -.70 & 25.0 & 1.3 & .10 & .10 & .30 & .50 & -1.20 \\
\hline 8131 & 3.1 & 22. & 130. & -.70 & 25.0 & 1.3 & .10 & .10 & .30 & .50 & -1.20 \\
\hline 8132 & 1.2 & 22 . & 130. & -.70 & 25.0 & 1.3 & .10 & .10 & .30 & .50 & -1.20 \\
\hline 8133 & 1.4 & 22. & 130. & -.70 & 25.0 & 1.3 & .10 & .10 & .30 & .50 & -1.20 \\
\hline 8140 & 3.5 & 22. & 130. & -.70 & 10.0 & 1.3 & .10 & .10 & .30 & .50 & -1.20 \\
\hline 8141 & 1.0 & 22. & 130. & -.70 & 10.0 & 1.3 & .10 & .10 & .30 & .50 & -1.20 \\
\hline 8206 & -1.20 & & .50 & .60 & & & & & & & \\
\hline 8207 & -1.20 & & .50 & .60 & & & & & & & \\
\hline 8208 & -1.20 & & .50 & .60 & & & & & & & \\
\hline 8209 & -1.20 & & .50 & .60 & & . & & & & & \\
\hline 8211 & -1.20 & & .50 & .60 & & & & & & & \\
\hline 8212 & -1.20 & & .50 & .60 & & & & & & & \\
\hline 8214 & -1.20 & & .50 & .60 & & & & & & & \\
\hline 8215 & -1.20 & & .50 & .60 & & & & & & & \\
\hline 8216 & -1.20 & & .50 & .60 & & & & & & & \\
\hline 8220 & -1.20 & & .50 & .60 & & & & & & & \\
\hline 8221 & -1.20 & & .50 & .60 & & & & & & & \\
\hline 8222 & -1.20 & & .50 & .60 & & & & & & & \\
\hline 8223 & -1.20 & & .50 & .60 & & & & & & & \\
\hline 8224 & -1.20 & & .50 & .60 & & & & & & & \\
\hline 8225 & -1.20 & & .50 & .60 & & & & & & & \\
\hline 8226 & -1.20 & & .50 & .60 & & & & & & & \\
\hline 8227 & -1.20 & & .50 & .60 & & & & & & & \\
\hline 8228 & -1.20 & & .50 & .60 & & & & & & & \\
\hline 8230 & -1.20 & & .50 & .60 & & & & & & & \\
\hline 8231 & -1.20 & & .50 & .60 & & & & & & & \\
\hline 8232 & -1.20 & & .50 & .60 & & & & & & & \\
\hline 8233 & -1.20 & & .50 & .60 & & & & & & & \\
\hline 8240 & -1.20 & & .50 & .60 & . & & & & & & \\
\hline 8241 & -1.20 & & .50 & .60 & & & & & & & \\
\hline 8307 & .0003 & & & & & & & & & 0 . & \\
\hline 8308 & .0032 & & & & & & & & & 0. & \\
\hline 8309 & .0031 & & & & & & & & & 0. & \\
\hline 8310 & .0006 & & & & & & & & & 0 . & \\
\hline 8311 & .0028 & & & & & & & & & 0 . & \\
\hline 8329 & 4.8 & 17.0 & 730. & .5 & 1. & & & & 1.8 & .1 & 730. \\
\hline 8330 & 4.9 & 17.0 & 730 & .5 & 1. & & & & 1.8 & .1 & 730 \\
\hline 8331 & .2 & 17.0 & 1080. & .5 & 1. & & & & .5 & .1 & 1080. \\
\hline 8332 & .4 & 17.0 & 1080 . & .5 & 1. & & & & .5 & .1 & 1080. \\
\hline 8333 & .3 & 17.0 & 1080. & .5 & 1. & & & & .5 & .1 & 1080. \\
\hline 8334 & .4 & 17.0 & 1080 . & .5 & 1. & & & & .5 & .1 & 1080 . \\
\hline 8335 & .9 & 17.0 & 2250 . & .5 & 1. & & & & .1 & .1 & 2250 . \\
\hline 8336 & .2 & 17.0 & 2250 . & .5 & 1. & & & & .1 & .1 & 2250 . \\
\hline 8337 & .2 & 17.0 & 2250 . & .5 & 1. & & & & .1 & .1 & 2250 \\
\hline
\end{tabular}


APPENDIX II--LISTING OF EXAMPLE DATA SETS--Continued.

\begin{tabular}{|c|c|c|c|c|c|c|c|c|c|c|}
\hline 8338 & .2 & 17.0 & 780. & .5 & 1. & & & 1.0 & .1 & 780 . \\
\hline 8339 & .2 & 17.0 & 780 & .5 & 1. & & & 1.0 & .1 & 780. \\
\hline 8340 & .7 & 17.0 & 780 & .5 & 1. & & & 1.0 & .1 & 780 \\
\hline 8341 & .2 & 17.0 & 780. & .5 & 1. & & & 1.0 & .1 & 780. \\
\hline 8342 & .7 & 17.0 & 780 & .5 & 1 . & & & 1.0 & .1 & 780 \\
\hline 8343 & .3 & 17.0 & 2410 . & .5 & 1. & & & 1.0 & .1 & 2410 . \\
\hline 8407 & & & 6600 & 6600 & & & & & & \\
\hline 8408 & & & 6600. & . 6600 . & & & & & & \\
\hline 8409 & & & 6600. & . 6600 . & & & & & & \\
\hline 8410 & & & 6600. & . 6600 . & & & & & & \\
\hline 8411 & & & 6600. & . 6600 . & & & & & & \\
\hline 8429 & 540 & & .1 & .1 & & & & & & \\
\hline 8430 & 540 & & .1 & .1 & & & & & & \\
\hline 8431 & 800 & & .1 & .1 & & & & & & \\
\hline 8432 & 800 & & .1 & .1 & & & & & & \\
\hline 8433 & 800 & & .1 & .1 & & & & & & \\
\hline 8434 & 800 & & .1 & .1 & & & & & & \\
\hline 8435 & 167 & & .1 & .1 & & & & & & \\
\hline 8436 & 167 & & .1 & .1 & & & & & & \\
\hline 8437 & 167 & & .1 & .1 & & & & & & \\
\hline 8438 & 580 & & .1 & .1 & & & & & & \\
\hline 8439 & 580 & & .1 & .1 & & & & & & \\
\hline 8440 & 580 & & .1 & .1 & & & & & & \\
\hline 8441 & 580 & & .1 & .1 & & & & & & \\
\hline 8442 & 580 & & .1 & .1 & & & & & & \\
\hline 8443 & 179 & & .1 & .1 & & & & & & \\
\hline 9156. & .12 & 9.7 & 126. & 3.0 & .69 & .06 & .02 & .00 & .04 & 130. \\
\hline 9156 & .12 & 8.3 & 102. & 2.5 & .52 & .03 & .02 & .00 & .02 & 126. \\
\hline 9156. & .12 & 6.8 & 82. & 1.9 & .34 & .01 & .01 & .00 & .01 & 124. \\
\hline 9152 & .23 & 8.5 & 115. & 7.3 & 1.7 & 1.1 & .08 & .14 & .76 & 253. \\
\hline 9152. & .23 & 7.2 & 91. & 6.2 & 1.7 & 1.1 & .06 & .11 & .46 & 240. \\
\hline 9152 & 23 & 6.0 & 74. & 5.4 & 1.7 & 1.1 & .04 & .07 & .20 & 215. \\
\hline 9150 & .05 & 8.0 & 107. & 6.7 & 1.4 & .76 & .14 & .31 & .77 & 254. \\
\hline 9150 & .05 & 6.9 & 88 & 5.6 & 1.4 & .76 & .10 & .26 & .42 & 246. \\
\hline 9150 & .05 & 5.6 & 68 & 4.9 & 1.4 & .76 & .03 & .21 & .14 & 234. \\
\hline 9144 & 92 & 9.2 & 128. & 6.5 & 1.1 & .54 & .31 & .82 & .99 & 268. \\
\hline 9144 & 92 & 7.1 & 93. & 5.7 & 1.1 & .54 & .24 & .63 & .65 & 251. \\
\hline 9144 & .92 & 5.4 & 68. & 4.5 & 1.1 & .54 & .10 & .26 & .35 & 240 . \\
\hline 9138 & 60 & 9.9 & 138. & 6.3 & 1.4 & .49 & .34 & 1.1 & .94 & 265. \\
\hline 9138 & 60 & 7.5 & 98. & 5.1 & 1.1 & .35 & .29 & .86 & .64 & 253. \\
\hline 9138 & .60 & 5.2 & 64. & 4.4 & .98 & .19 & .21 & .45 & .37 & 239. \\
\hline 9134 & .88 & 9.0 & 122. & 6.7 & 2.0 & .34 & .39 & 1.2 & .99 & 272. \\
\hline 9134. & .88 & 6.7 & 87. & 5.4 & 1.4 & .25 & .30 & 1.1 & .66 & 260. \\
\hline 9134 & .88 & 5.5 & 69. & 4.6 & .90 & .12 & .24 & .96 & .40 & 256. \\
\hline 9134 & .52 & 8.0 & & & & & & & & \\
\hline 9134 & .52 & 7.0 & & & & & & & & \\
\hline 9134 & .52 & 5.7 & & & & & & & & \\
\hline 9129 & .97 & 9.9 & 138. & 5.4 & 2.4 & .10 & .24 & 1.2 & .77 & 270 . \\
\hline 9129 & .97 & 8.4 & 113. & 4.8 & 1.8 & .07 & .15 & .92 & .55 & 263. \\
\hline 9129 & .97 & 6.3 & 81 & 4.1 & 1.2 & .04 & .08 & .64 & .34 & 252. \\
\hline
\end{tabular}


APPENDIX II--LISTING OF EXAMPLE DATA SETS--Continued.

\begin{tabular}{|c|c|c|c|c|c|c|c|c|c|c|}
\hline $9123^{\circ} .65$ & 13.1 & 188. & 7.0 & & 1.6 & .06 & .09 & .52 & .46 & 274. \\
\hline 9123.65 & 8.6 & 115. & 5.5 & & .99 & .04 & .07 & .38 & .33 & 264. \\
\hline 9123.65 & 5.0 & 64. & 3.8 & & .68 & .02 & .02 & .29 & .24 & 254. \\
\hline 9113.18 & 11.2 & 158. & 6.7 & & 1.0 & .07 & .02 & .00 & .28 & 406. \\
\hline 9113.18 & 7.6 & 101. & 5.9 & & .80 & .04 & .01 & .00 & .22 & 384. \\
\hline 9113.18 & 4.8 & 59. & 4.8 & & .64 & .00 & .01 & .00 & .15 & 353. \\
\hline 913.22 & 9.2 & 130. & 4.5 & & 1.4 & .07 & .02 & .00 & .28 & 532. \\
\hline 913.22 & 7.5 & 99. & 4.1 & & 1.1 & .04 & .01 & .00 & .21 & 517. \\
\hline 913.22 & 5.9 & 73. & 3.6 & & .84 & .01 & .00 & .00 & .13 & 477. \\
\hline 910 & 10.4 & 132. & 3.7 & & 1.3 & .10 & .01 & .00 & .25 & 557. \\
\hline 910. & 7.4 & 92. & 3.4 & & 1.0 & .05 & .00 & .00 & .21 & 533. \\
\hline 910. & 5.2 & 66. & 2.8 & & .54 & .02 & .00 & .00 & .13 & 511. \\
\hline 9288. & & .04 & .11 & 165. & .007 & .18 & 480. & & & \\
\hline 9285. & & .02 & .07 & 155. & .002 & .18 & 390 . & & & \\
\hline 9284. & & .01 & .04 & 145. & .000 & .18 & 300 & & & \\
\hline 92159. & & .76 & .79 & 320 . & .099 & .36 & 1200. & & & \\
\hline 92152 . & & .46 & .51 & 300. & .051 & .36 & 1040 . & & & \\
\hline 92138. & & .20 & .30 & 290. & .008 & .36 & 690. & & & \\
\hline 92160. & & .77 & .81 & 310 . & .032 & .52 & 1340. & & & \\
\hline 92155. & & .42 & .48 & 285. & .023 & .52 & 1170 . & & & \\
\hline 92149. & & .14 & .20 & 275 . & .003 & .52 & 950. & & & \\
\hline 92168. & & .99 & 1.0 & 285. & .076 & 1.4 & 1810 . & & & \\
\hline 92158 . & & .65 & .65 & 280. & .017 & 1.0 & 1440 . & & & \\
\hline 92152. & & .35 & .39 & 280. & .003 & .64 & 1100. & & & \\
\hline 92166. & & .94 & .97 & 280. & .065 & 1.3 & 2640 . & & & \\
\hline 92159. & & .64 & .68 & 275 . & .014 & .75 & 1960. & & & \\
\hline 92151. & & .37 & .40 & 270. & .002 & .24 & 1670. & & & \\
\hline 92170. & & .99 & 1.0 & 285 . & .076 & 1.0 & 3330 . & & & \\
\hline 92163. & & .66 & .72 & 270 . & .029 & .84 & 2630 . & & & \\
\hline 92161. & & .40 & .47 & 265. & .01 & .68 & 2110 . & & & \\
\hline 92 & & & & 70. & .000 & & & & & \\
\hline 92 & & & & 65. & .000 & & & & & \\
\hline 92 & & & & 60. & .000 & & & & & \\
\hline 92169. & & .77 & .80 & 70. & .042 & 1.4 & 3190 . & & & \\
\hline 92165. & & .55 & .60 & 70. & .013 & 1.0 & 2710 . & & & \\
\hline 92159. & & .34 & .45 & 70. & .005 & .64 & 1740 . & & & \\
\hline 92171. & & .46 & .50 & 60. & .007 & 4.1 & 4240 . & & & \\
\hline 92166. & & .33 & .40 & 55. & .005 & 2.7 & 3230 . & & & \\
\hline 92160. & & .24 & .29 & 50. & .001 & 1.4 & 2020 . & & & \\
\hline 92247. & & .28 & .31 & 60. & .028 & 1.2 & 4040 . & & & \\
\hline 92234 . & & .22 & .28 & 55. & .007 & 1.1 & 3560 . & & & \\
\hline 92216 & & .15 & .24 & 50. & .000 & .98 & 3150 . & & & \\
\hline 92318. & & .28 & .29 & 50. & .007 & .87 & 4730 . & & & \\
\hline 92310 & & .21 & .24 & 44. & .003 & .61 & 4230 . & & & \\
\hline 92287. & & .13 & .16 & 40. & .000 & .35 & 3520 . & & & \\
\hline 92332 . & & .25 & .30 & 55. & .005 & 1.6 & 5430 . & & & \\
\hline 92319. & & .21 & .26 & 45. & .005 & 1.1 & 4390 . & & & \\
\hline 92306. & & .13 & .20 & 40. & .002 & .66 & 3880 . & & & \\
\hline
\end{tabular}


APPENDIX II--LISTING OF EXAMPLE DATA SETS--Continued.

E. - Truckee Canal, June 1979

The following data set represents Truckee Canal conditions observed during field investigations in June 1979. Starting flows and concentrations for the following Truckee Canal data sets are those observed at the Derby Dam diversion into the canal.

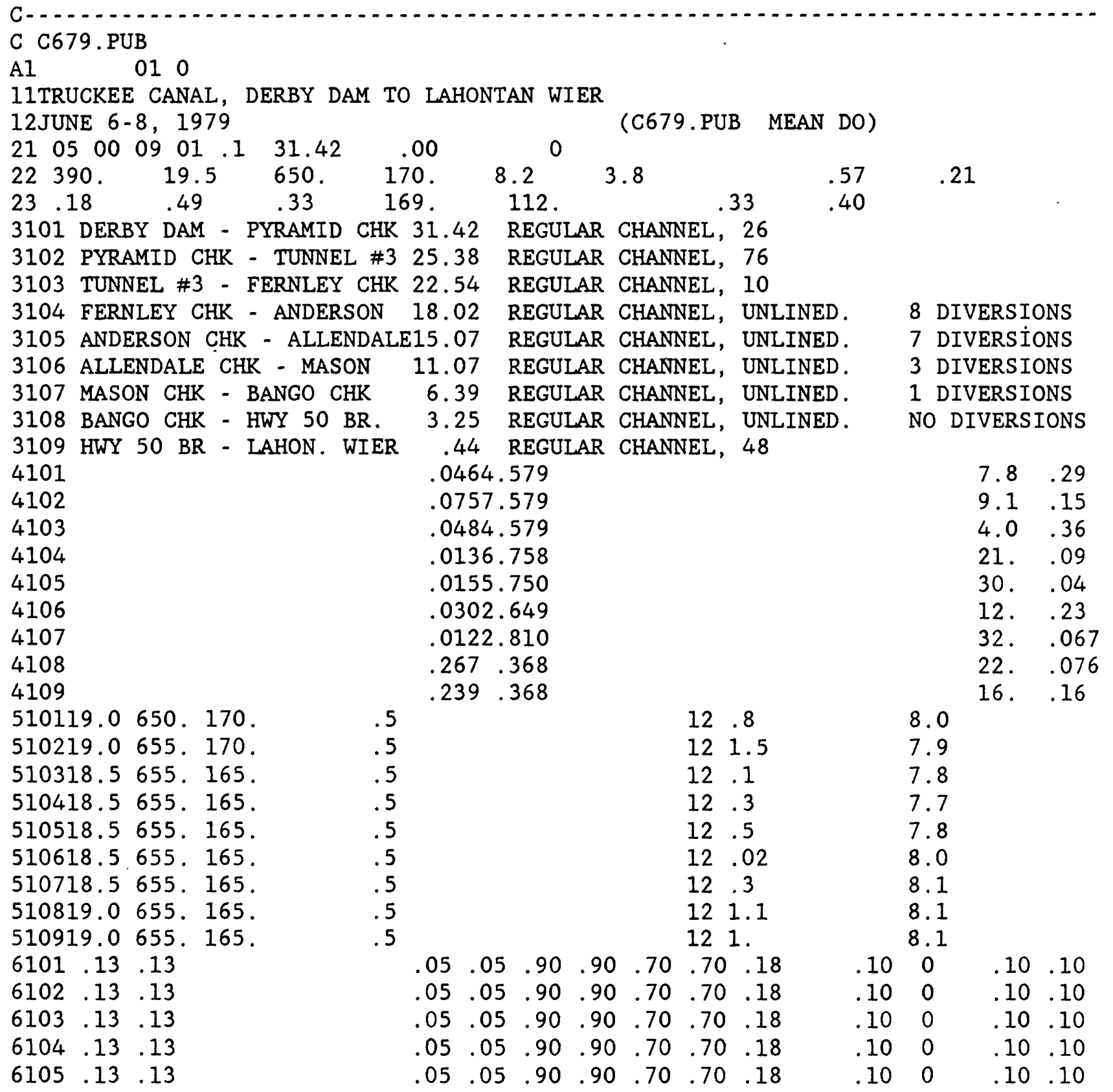


APPENDIX II--LISTING OF EXAMPLE DATA SETS--Continued.

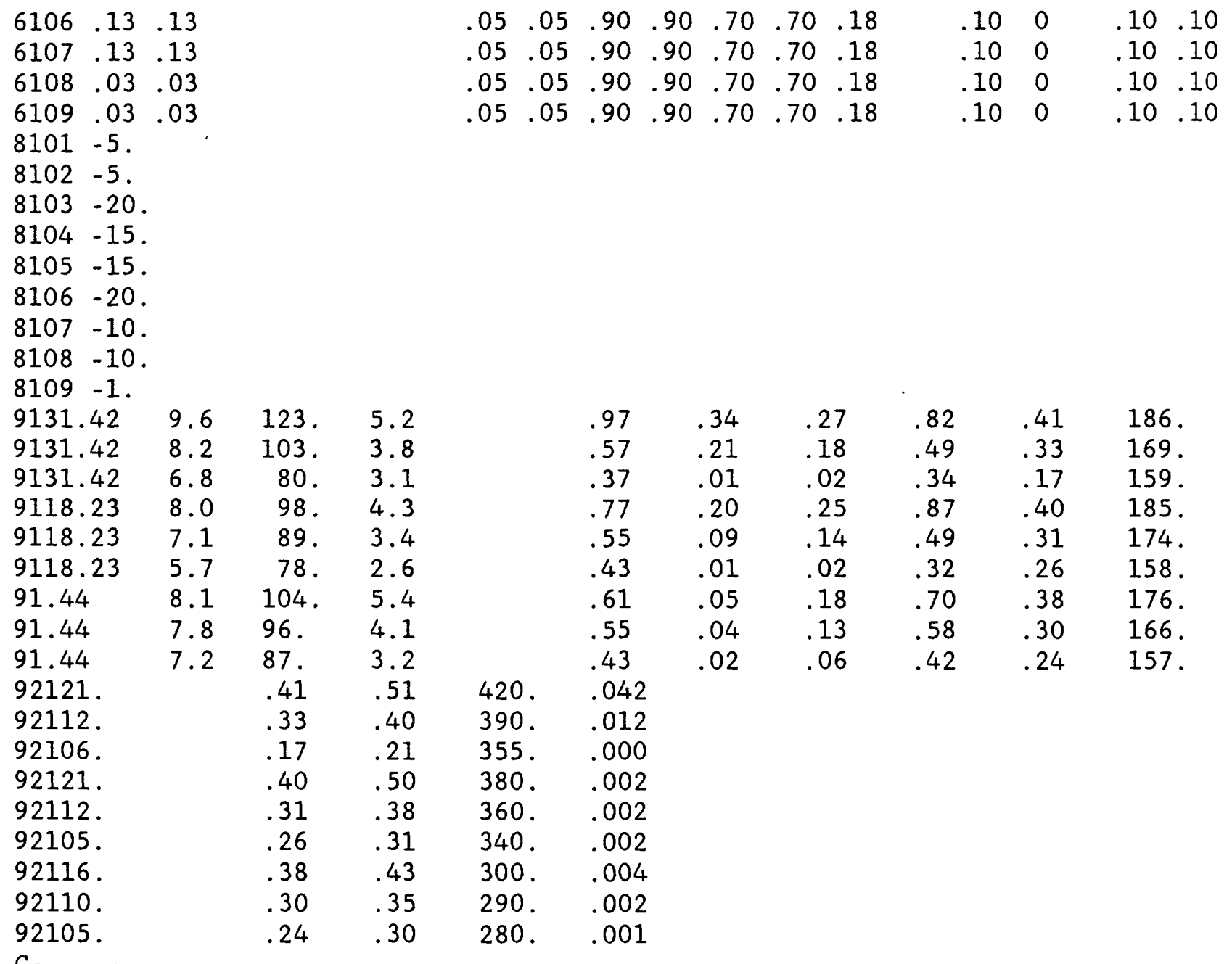


APPENDIX II--LISTING OF EXAMPLE DATA SETS--Continued.

F.--Truckee Canal, August 1979

The following data set represents Truckee Canal conditions observed during field investigations in August 1979.

\section{C879.PUB}

Al 010

11TRUCKEE CANAL, DERBY DAM TO LAHONTAN WIER

12AUGUST $9-10,1979$

$\begin{array}{lllllllll}22 & 220 . & 22.8 & 655 . & 235 . & 6.3 & 4.4 & & .68 \\ 23 & .19 & 1.1 & .69 & 237 . & 150 . & & .69 & .78\end{array}$

(C879.PUB MEAN DO)

3101 DERBY DAM - PYRAMID CHK 31.42 REGULAR CHANNEL, 26

3102 PYRAMID CHK - TUNNEL \#3 25.38 REGULAR CHANNEL, 76

3103 TUNNEL \#3 - FERNLEY CHK 22.54 REGULAR CHANNEL, 10

3104 FERNLEY CHK - ANDERSON 18.02 REGULAR CHANNEL, UNLINED. 8 DIVERSIONS

3105 ANDERSON CHK - ALLENDALE15.07 REGULAR CHANNEL, UNLINED. 7 DIVERSIONS

3106 ALLENDALE CHK - MASON 11.07 REGULAR CHANNEL, UNLINED. 3 DIVERSIONS

3107 MASON CHK - BANGO CHK 6.39 REGULAR CHANNEL, UNLINED. 1 DIVERSIONS

3108 BANGO CHK - HWY 50 BR. 3.25 REGULAR CHANNEL, UNLINED. NO DIVERSIONS

3109 HWY 50 BR - LAHON. WIER .44 REGULAR CHANNEL, 48

4101

4102

.0464 .579

.0757 .579

.0484 .579

4103

.0136 .758

4105

.0155 .750

4106

.0302 .649

4107

.0122 .810

4108

.267 .368

4109

510123.0655 .235$. $.239 \quad .368$

$\begin{array}{ll}7.8 & .29\end{array}$

$9.1 \quad .15$

$4.0 \quad .36$

21. .09

30. .04

12. . 23

32. . .067

22. .076

510223.0655 . 240.

2.5

510323.5655 .245$.

2.5

2.5

510423.5655 .245$.

2.5

510523.5655 .245$.

2.5

510623.5660 .245 .

2.5

510724.0660 . 245.

2.5

510824.0660 .245$.

2.5

510924.0660 . 245.

2.5

12.80

121.5

12.10

12.30

12.50

12.02

12.30

121.1

121.0

$\begin{array}{llllll}.05 & .05 & .90 & .90 & .70 & .70 \quad .18\end{array}$

$\begin{array}{llllllll}.05 & .05 & .90 & .90 & .70 & .70 & .18\end{array}$

$\begin{array}{lllllll}.05 & .05 & .90 & .90 & .70 & .70 & .18\end{array}$

$\begin{array}{lllllll}.05 & .05 & .90 & .90 & .70 & .70 & .18\end{array}$

$\begin{array}{lllllll}.05 & .05 & .90 & .90 & .70 & .70 & .18\end{array}$

$\begin{array}{lllllll}.05 & .05 & .90 & .90 & .70 & .70 & .18\end{array}$

$\begin{array}{lllllll}.05 & .05 & .90 & .90 & .70 & .70 & .78\end{array}$

16. . 16

8.1

8.2

8.2

8.3

8.5

8.8

8.9

9.0

9.0

$\begin{array}{llll}.10 & 0 & .10 & .10\end{array}$

$\begin{array}{llll}.10 & 0 & .10 & .10\end{array}$

$\begin{array}{llll}.10 & 0 & .10 & .10\end{array}$

$\begin{array}{llll}.10 & 0 & .10 & .10\end{array}$

$\begin{array}{llll}.10 & 0 & .10 & .10\end{array}$

$\begin{array}{llll}.10 & 0 & .10 & .10\end{array}$

$\begin{array}{llll}.10 & 0 & .10 & .10\end{array}$ 
APPENDIX II--LISTING OF EXAMPLE DATA SETS--Continued.

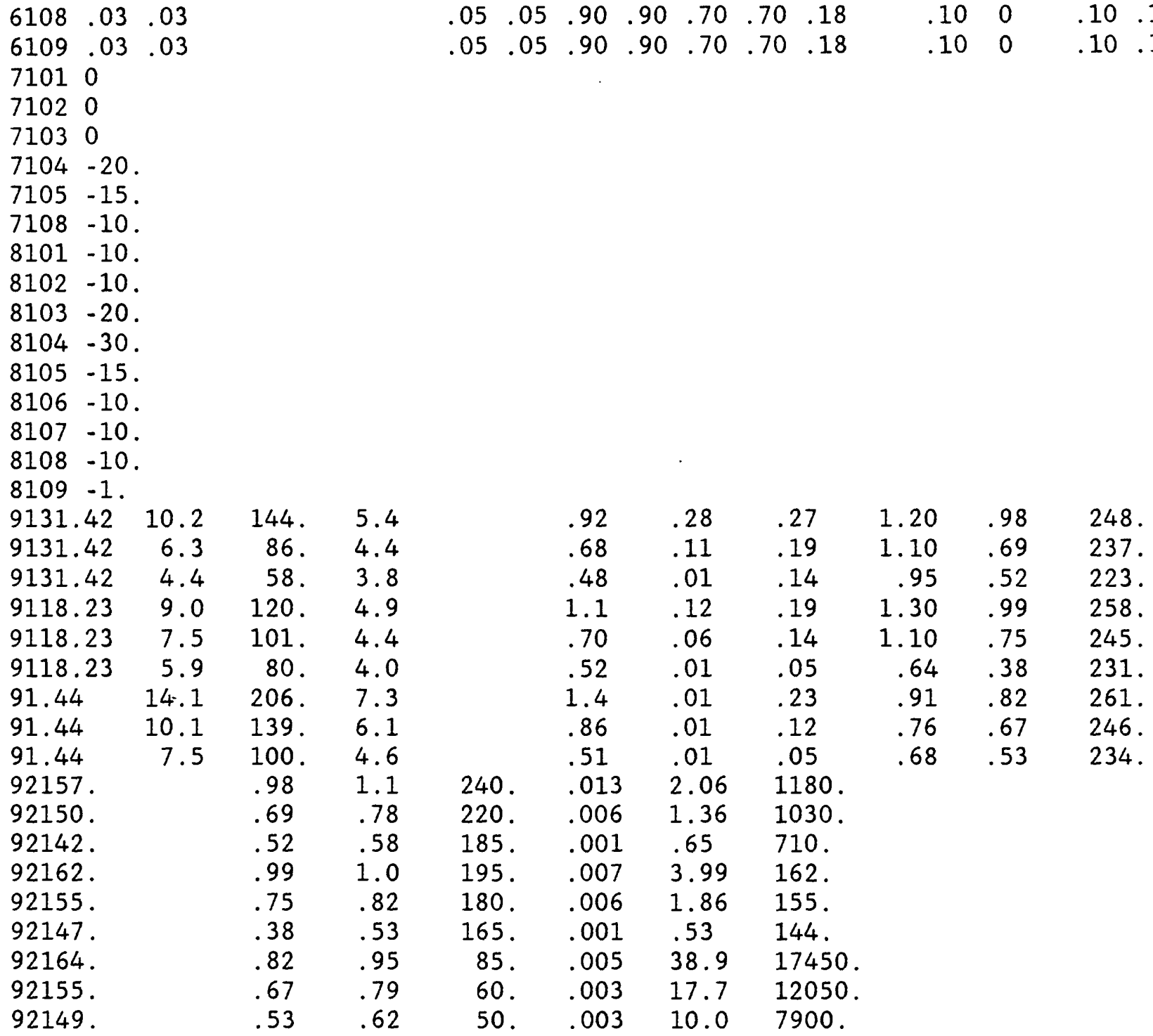


APPENDIX II--LISTING OF EXAMPLE DATA SETS--Continued.

G. - Truckee Canal, June 1980

The following data set represents Truckee Canal conditions observed during field investigations in June 1980.

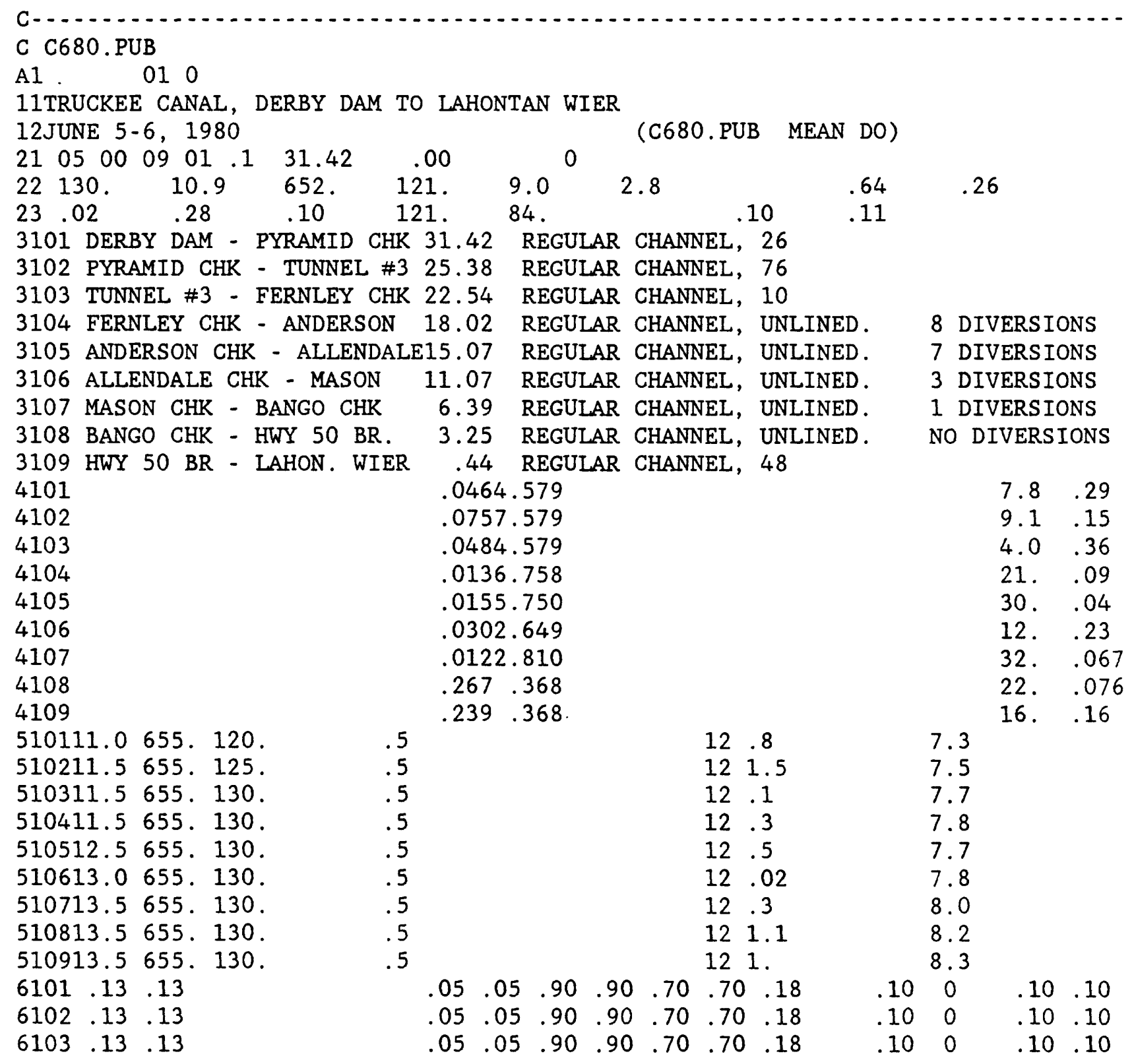


APPENDIX II--LISTING OF EXAMPLE DATA SETS--Continued.

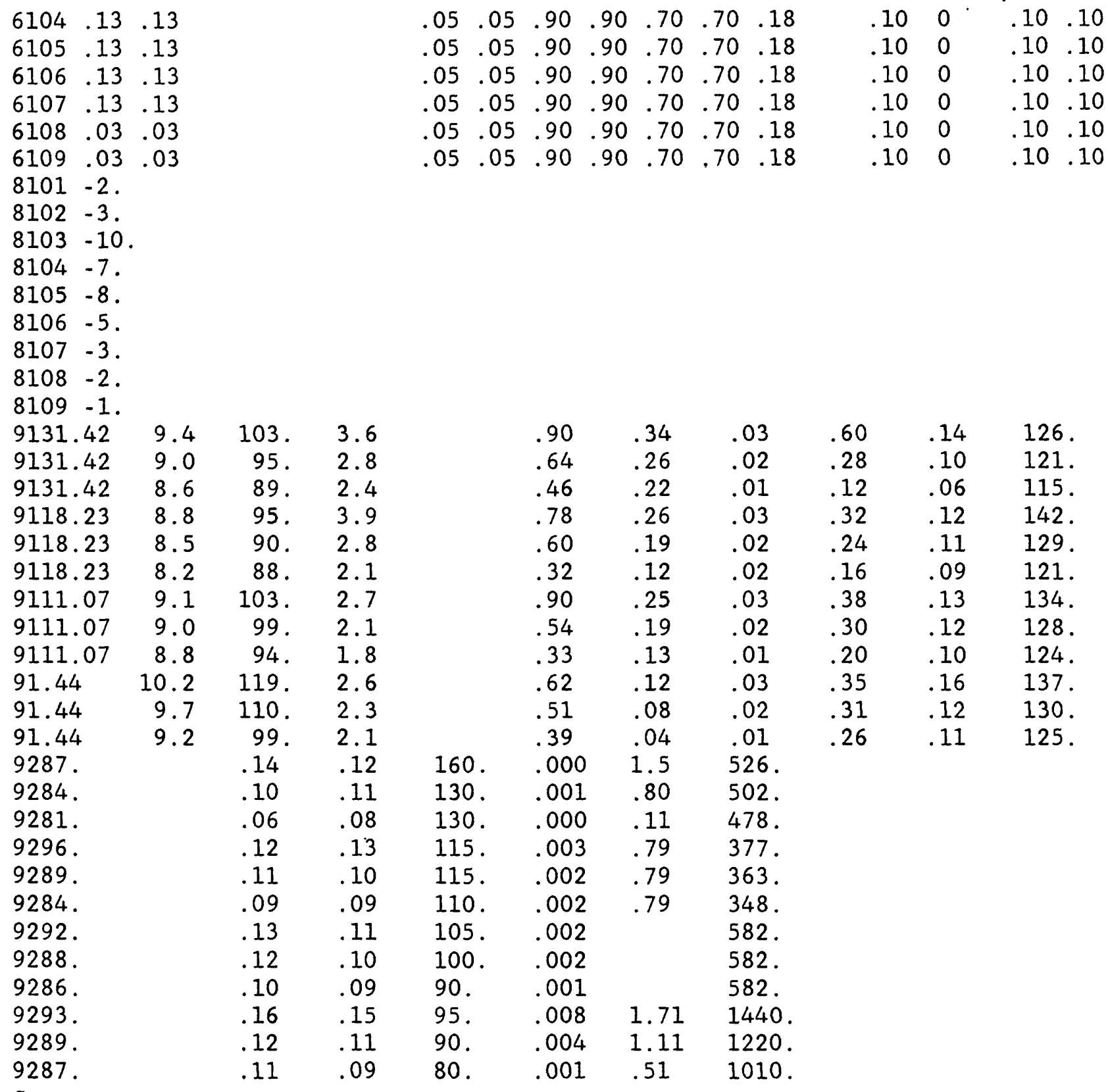

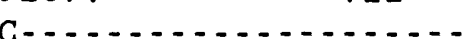


APPENDIX II--LISTING OF EXAMPLE DATA SETS--Continued.

H. - Truckee Canal, August 1980

The following data set represents Truckee Canal conditions observed during field investigations in August 1980.

C $\mathrm{C} 880$. PUB

A1 010

IITRUCKEE CANAL, DERBY DAM TO LAHONTAN WIER

12AUGUST 13-14, 1980

$\begin{array}{llllllll}21 & 05 & 00 & 09 & 01 & .1 & 31.42 & .00\end{array}$

22 205. $20.6 \quad 650.260$.

$\begin{array}{llll}23 & .30 & 1.10 \quad .66 \quad 260 .\end{array}$

3101 DERBY DAM - PYRAMID CHK 31.42 REGULAR CHANNEL, 26

3102 PYRAMID CHK - TUNNEL \#3 25.38 REGULAR CHANNEL, 76

3103 TUNNEL \#3 - FERNLEY CHK 22.54 REGULAR CHANNEL, 10

3104 FERNLEY CHK - ANDERSON 18.02 REGULAR CHANNEL, UNLINED. 8 DIVERSIONS

3105 ANDERSON CHK - ALLENDALE15.07 REGULAR CHANNEL, UNLINED. 7 DIVERSIONS

3106 ALLENDALE CHK - MASON 11.07 REGULAR CHANNEL, UNLINED. 3 DIVERSIONS

3107 MASON CHK - BANGO CHK 6.39 REGULAR CHANNEL, UNLINED. 1 DIVERSIONS

3108 BANGO CHK - HWY 50 BR. 3.25 REGULAR CHANNEL, UNLINED. NO DIVERSIONS

3109 HWY 50 BR - LAHON. WIER .44 REGULAR CHANNEL, 48

4101

4102

.0464 .579

.0757 .579

.0484 .579

.0136 .758

.0155 .750

.0302 .649

.0122 .810

1.4

.72

.25

4104

4105

4106

4107

.267 .368

4108

4109

$510121.0 \quad 650.235$.

$.239 \quad .368$

$\begin{array}{ll}7.8 & .29\end{array}$

$9.1 \quad .15$

$4.0 \quad .36$

21. .09

30. .04

12. .23

32. .067

22. .076

510221.0650 .240$.

2.5

2.5

12.80

16. .16

510321.5650 .245$.

2.5

121.5

8.2

510421.5650 .245$.

2.5

12.10

7.9

7.6

510522.0650 .245$.

2.5

12.30

12.50

7.4

510622.0650 .245$.

2.5

12.02

7.9

510722.0650 .245$.

2.5

12.30

8.2

510821.5650 .245$.

2.5

121.1

8.3

510921.5650 .245$.

2.5

121.0

8.4

6101.13 .13

6102.13 .13

6103.13 .13

6104.13 .13

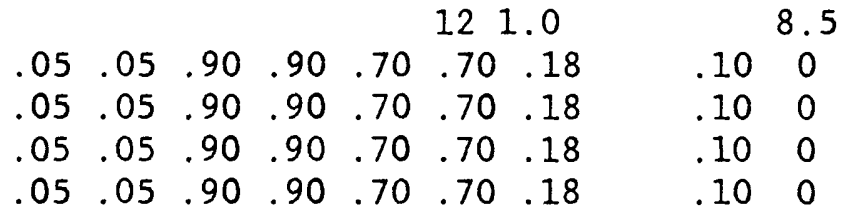

.10 .10

.10 .10

.10 .10

$.10 \quad .10$ 
APPENDIX II--LISTING OF EXAMPLE DATA SETS--Continued.

\begin{tabular}{|c|c|c|c|c|c|c|c|c|c|c|c|c|c|}
\hline 6105 . & .13 & .13 & & & .05 & .05 & .90 & .90 & .70 & .70 & .10 & 0 & .10 .10 \\
\hline 6106 . & .13 & .13 & & & .05 & .05 & .90 & .90 & .70 & .70 & .10 & 0 & .10 \\
\hline 6107 & .13 & .13 & & & .05 & .05 & .90 & .90 & .70 & .70 & .10 & 0 & .10 \\
\hline 6108. & .03 & .03 & & & .05 & .05 & .90 & .90 & .70 & .70 & .10 & 0 & .10 \\
\hline 6109 & .03 & .03 & & & .05 & .05 & .90 & .90 & .70 & .70 & .10 & 0 & .10 .10 \\
\hline 7105 & -15 & & & & & & & & & & & & \\
\hline 7106 & -5 & & & & & & & & & & & & \\
\hline 7108 & -15. & & & & & & & & & & & & \\
\hline 8101 & -10. & & & & & & & & & & & & \\
\hline 8102 & -5 & & & & & & & & & & & & \\
\hline 8103 & -40 & & & & & & & & & & & & \\
\hline 8104 & -40 & & & & & & & & & & & & \\
\hline 8105 & -25. & & & & & & & & & & & & \\
\hline 8106 & -10. & & & & & & & & & & & & \\
\hline 8107 & -10 & & & & & & & & & & & & \\
\hline 8108 & -10 & & & & & & & & & & & & \\
\hline 8109 & -1 & & & & & & & & & & & & \\
\hline 9131.4 & 42 & 9.0 & 122. & 6.7 & & & 2.0 & & 34 & .39 & 1.2 & .99 & 272 . \\
\hline 9131.4 & 42 & 6.7 & 87. & 5.4 & & & 1.4 & & 25 & .30 & 1.1 & .66 & 260 . \\
\hline 9131.4 & 42 & 5.5 & 69. & 4.6 & & & .90 & & 12 & .24 & .96 & .40 & 256. \\
\hline 9118.2 & 23 & 7.0 & 114. & 5.4 & & & 1.5 & & .15 & .31 & 1.3 & .86 & 251. \\
\hline 9118.2 & 23 & 6.8 & 92. & 4.3 & & & 1.0 & & .11 & .23 & 1.2 & .56 & 245 . \\
\hline 9118.2 & 23 & 6.4 & 85 & 3.5 & & & .69 & & .04 & .15 & .93 & .30 & 238. \\
\hline 9111. & 07 & 9.8 & 132. & 5.6 & & & 1.5 & & .07 & .26 & 1.3 & .58 & 242. \\
\hline 9111. & 07 & 8.8 & 118. & 4.8 & & & .99 & & .03 & .19 & 1.2 & .45 & 234. \\
\hline 9111. & 07 & 7.8 & 103. & 4.1 & & & .74 & & .00 & .05 & 1.1 & .36 & 225. \\
\hline 91.44 & & 14.2 & 206. & 4.4 & & & 1.1 & & .06 & .13 & 1.10 & .37 & 226. \\
\hline 91.44 & & 10.2 & 137. & 3.9 & & & .88 & & .03 & .09 & .78 & .30 & 217. \\
\hline 91.44 & & 7.8 & 98. & 3.5 & & & .57 & & .00 & .04 & .44 & .20 & 210. \\
\hline 92170 & & & .99 & 1.0 & 215 & & .076 & & 1.0 & 3330. & & & \\
\hline 92163 & & & .66 & .72 & 205 & & .029 & & .84 & 2630 . & & & \\
\hline 92161 & & & .40 & .47 & 205 & & .001 & & .68 & 2110 . & & & \\
\hline 92158 & & & .86 & .87 & 165 & & .002 & & .99 & 3620 . & & & \\
\hline 92155 & & & .56 & .60 & 150 & & .001 & & .67 & 2990. & & & \\
\hline 92151 & & & .30 & .35 & 145 & & .000 & & .35 & 2450 . & & & \\
\hline 92153 & & & .58 & .62 & 90 & & .005 & & 3.4 & 11300 . & & & \\
\hline 92149 & & & .45 & .52 & 70 & & .002 & & 2.1 & 8950 & & & \\
\hline 92144 & & & .36 & .43 & 50 & & .000 & & .79 & 6400 . & & & \\
\hline 92144 & & & .37 & .43 & 25 & & .019 & & 2.8 & 5390. & & & \\
\hline 92139 & & & .30 & .35 & 20 & & .004 & & 1.5 & 3950 . & & & \\
\hline 92135 & & & .20 & .26 & 15 & & .000 & & .16 & 2340 . & & & \\
\hline
\end{tabular}

$\begin{array}{lll}9.0 & 122 . & 6.7\end{array}$

$6.7 \quad 87 . \quad 5.4$

$5.5 \quad 69.4 .6$

7.0 114. 5.4

$6.8 \quad 92.4 .3$

$6.4 \quad 85.3 .5$

$9.8 \quad 132 . \quad 5.6$

118. 4.8

4.1

3.5

47 


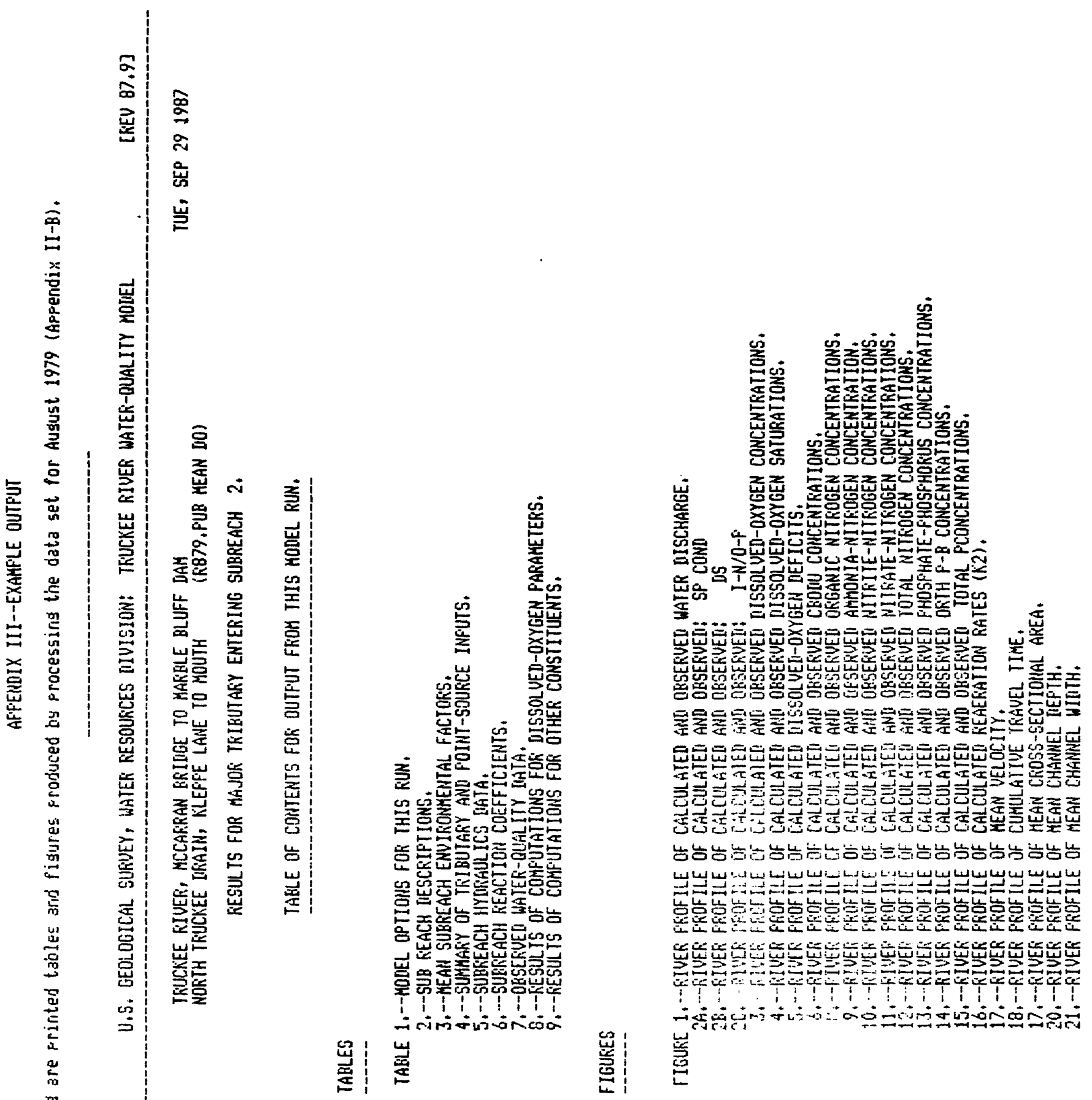




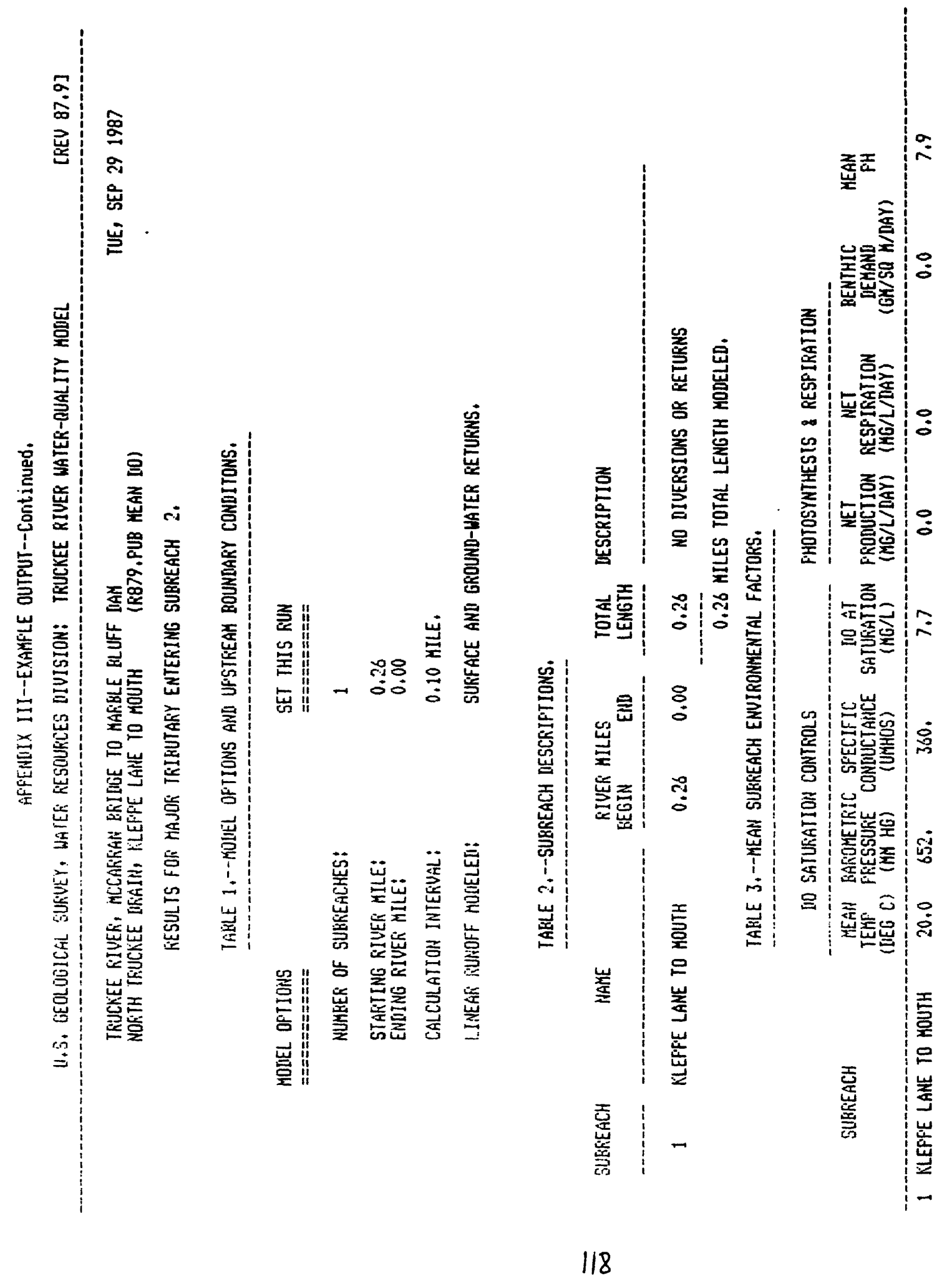




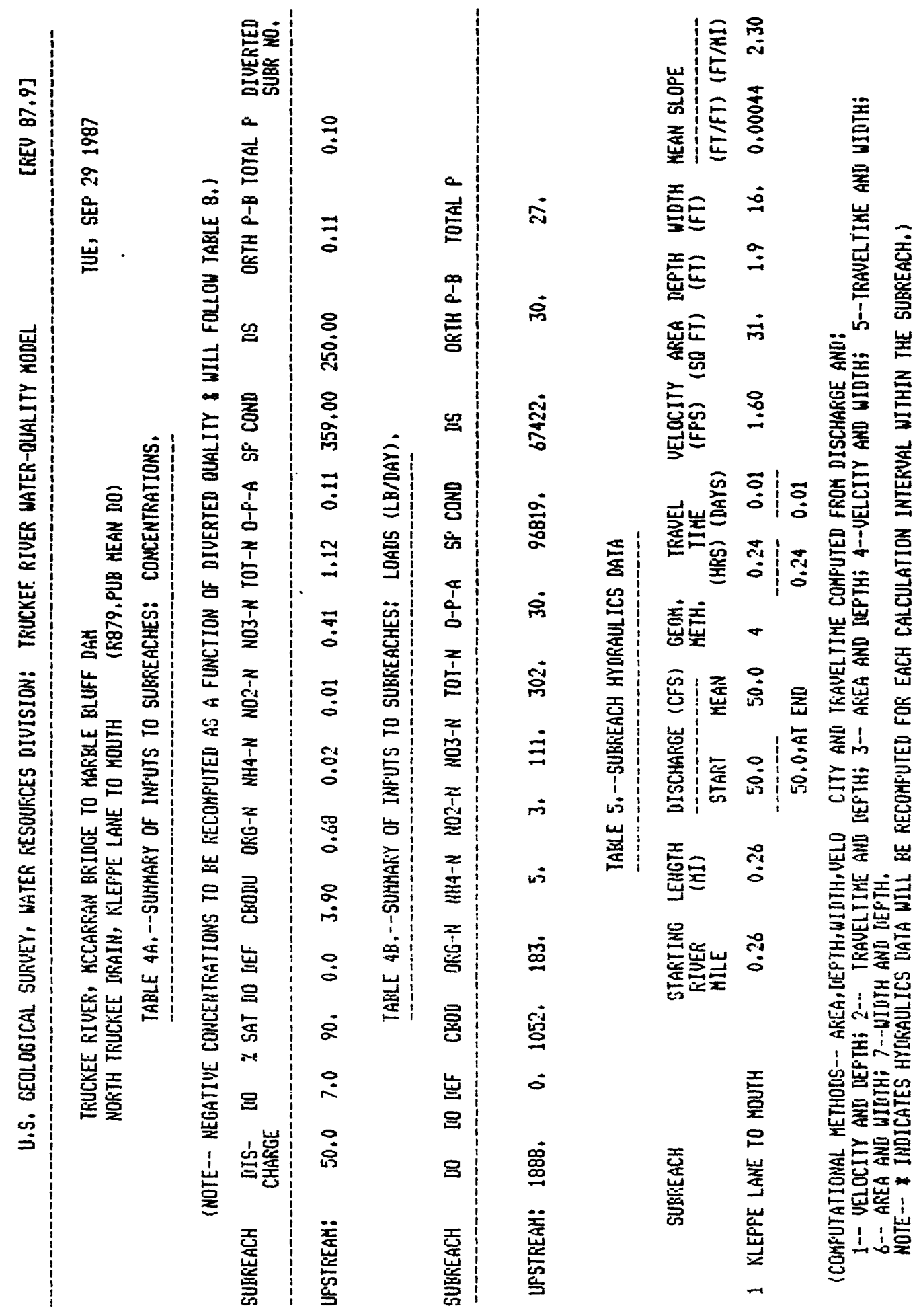



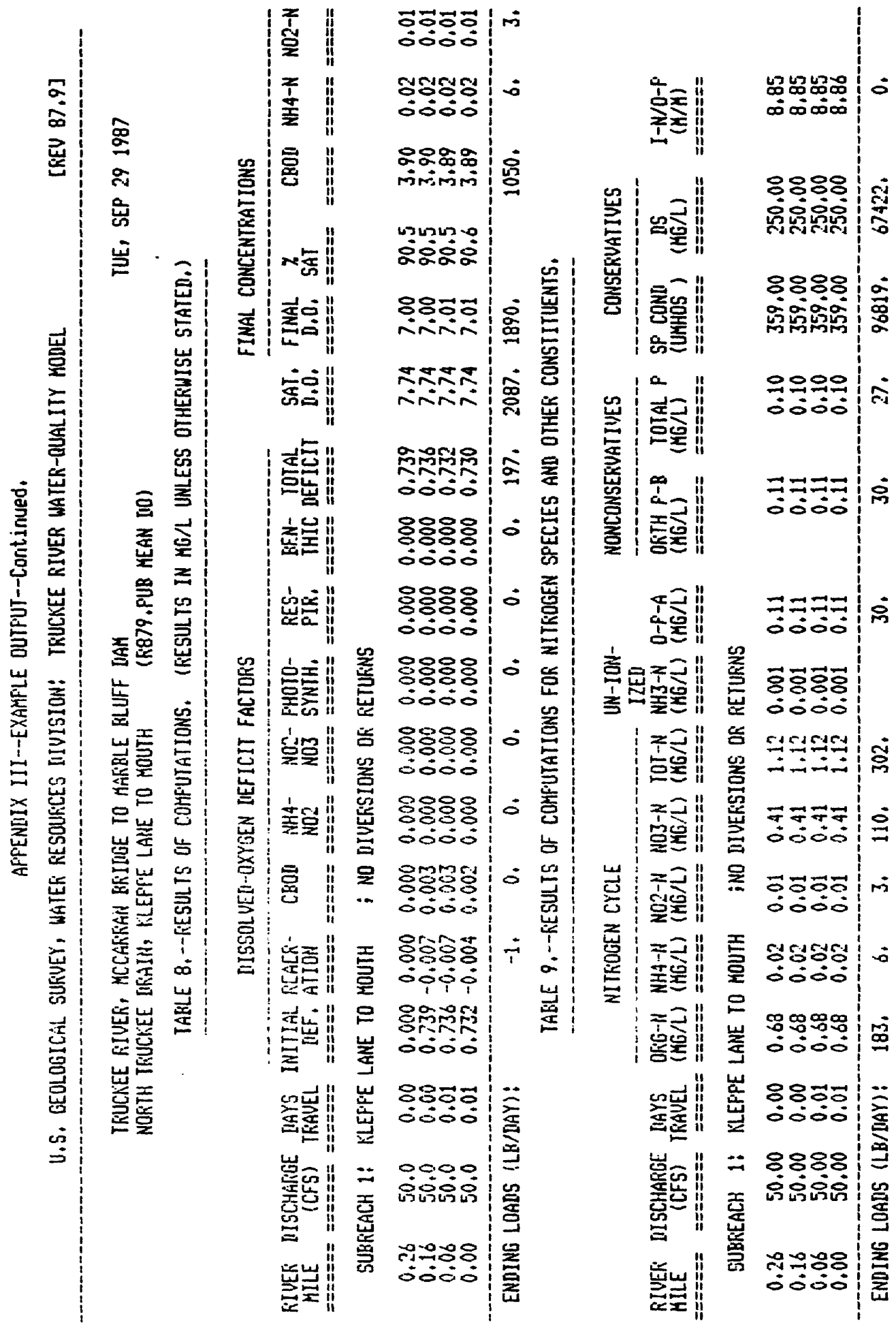


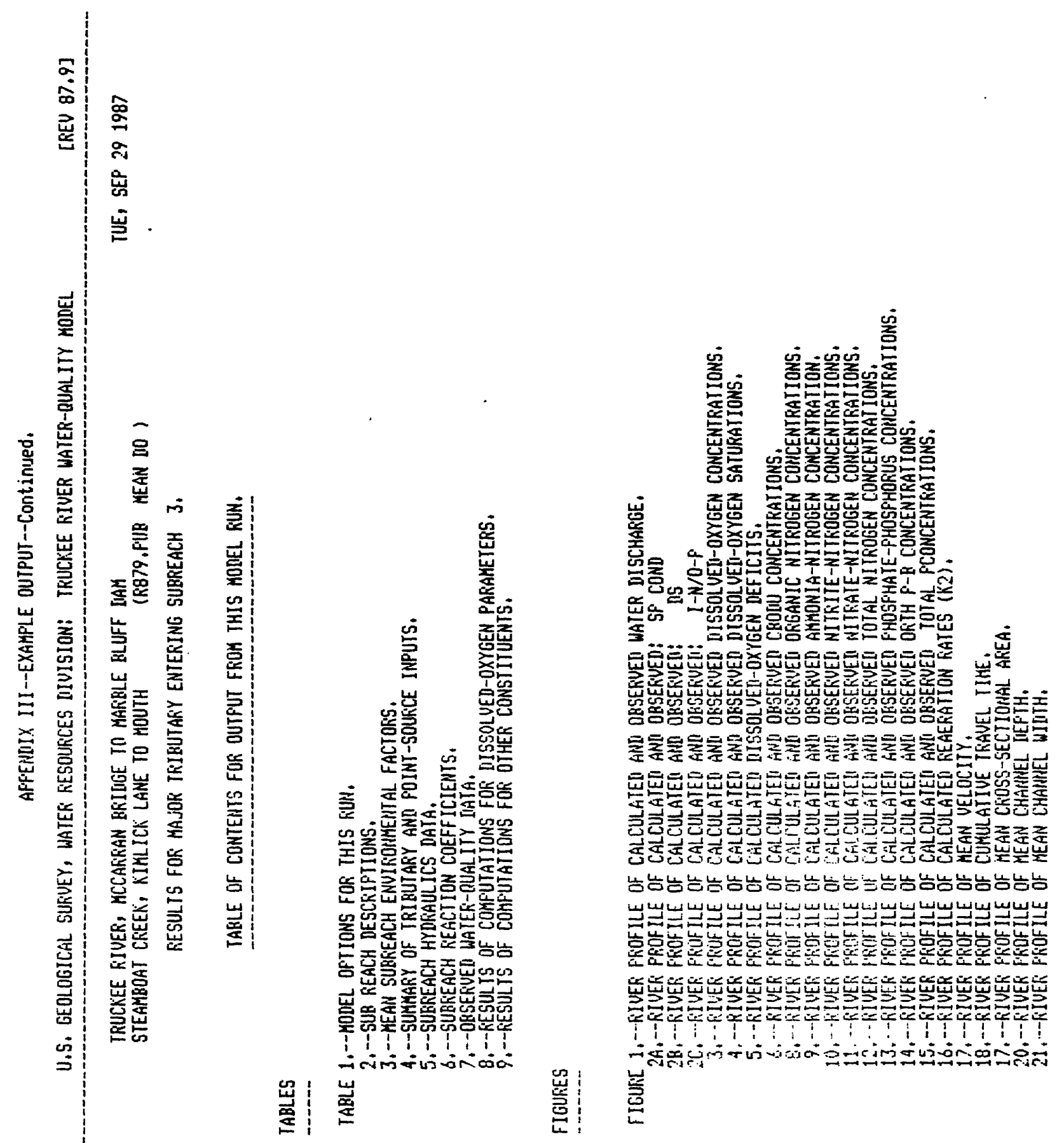




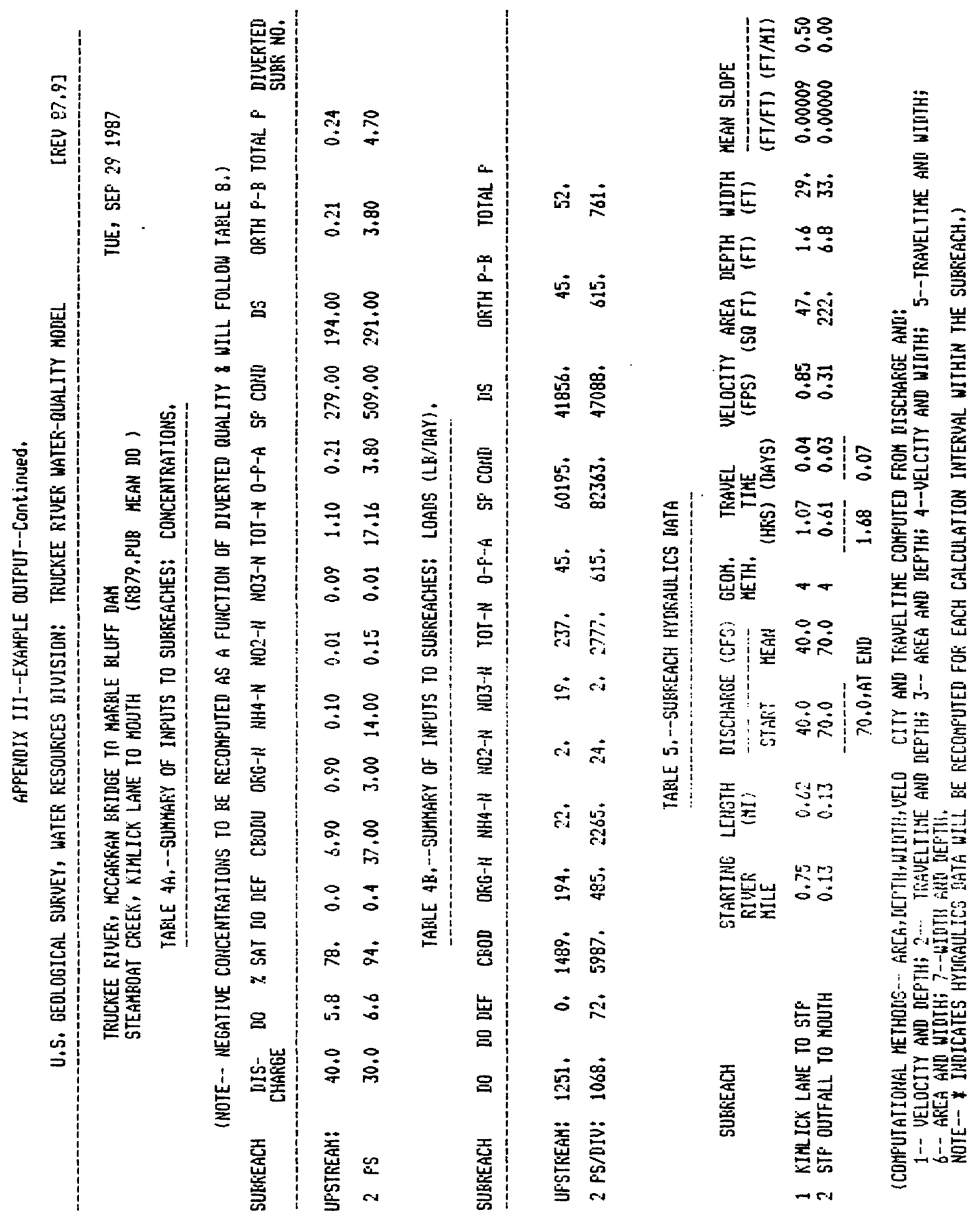




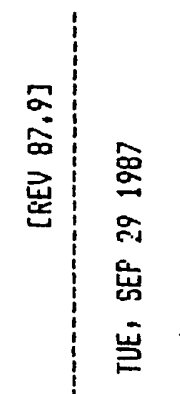

崖

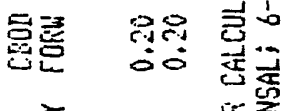

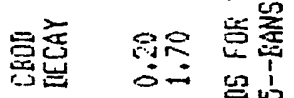

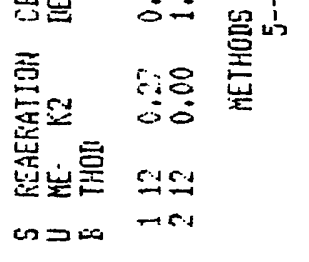

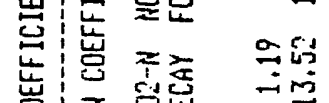

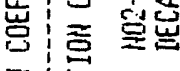

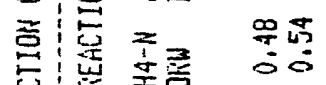

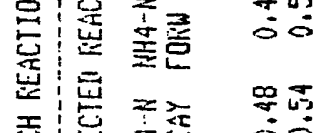

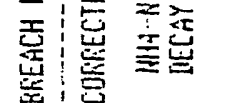

寒 岩

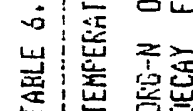

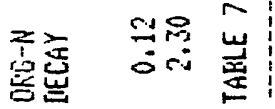

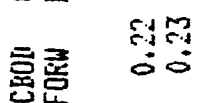

홀 경영

ऽ

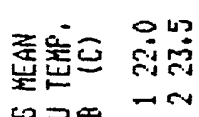

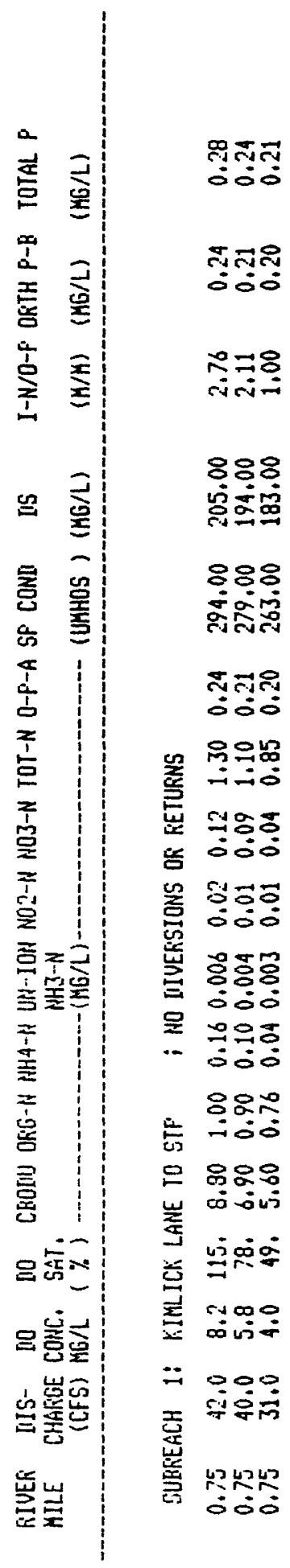



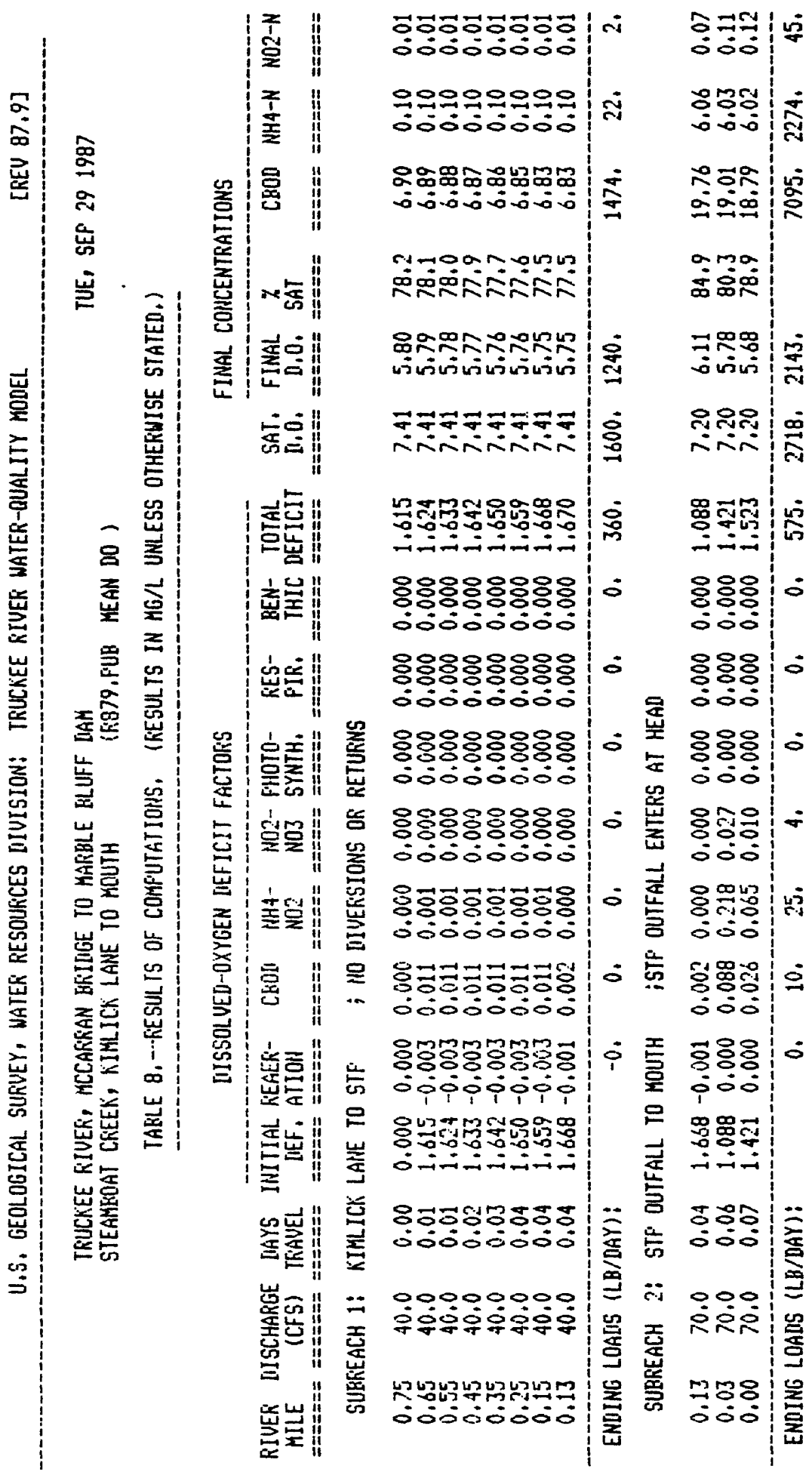


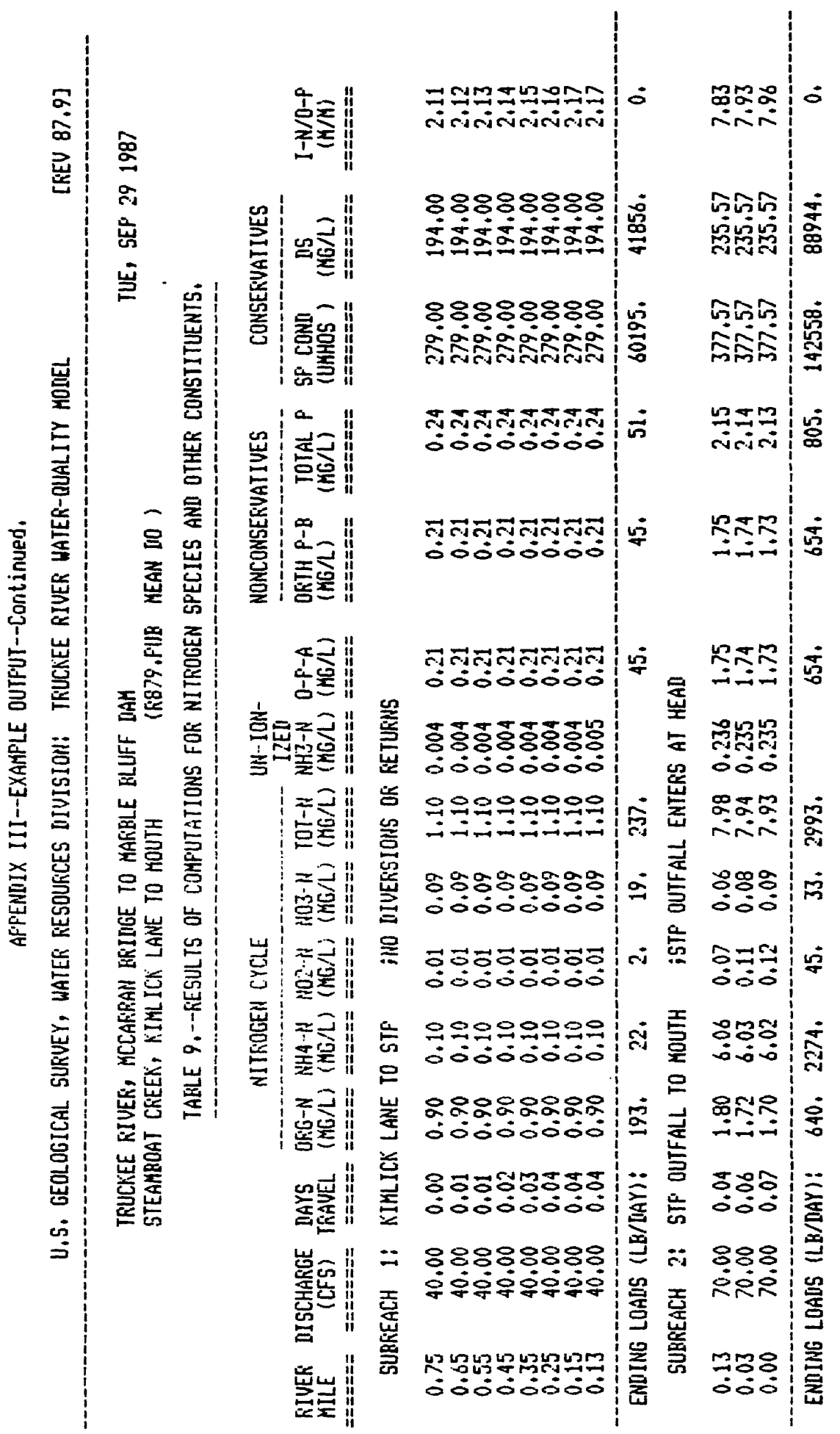




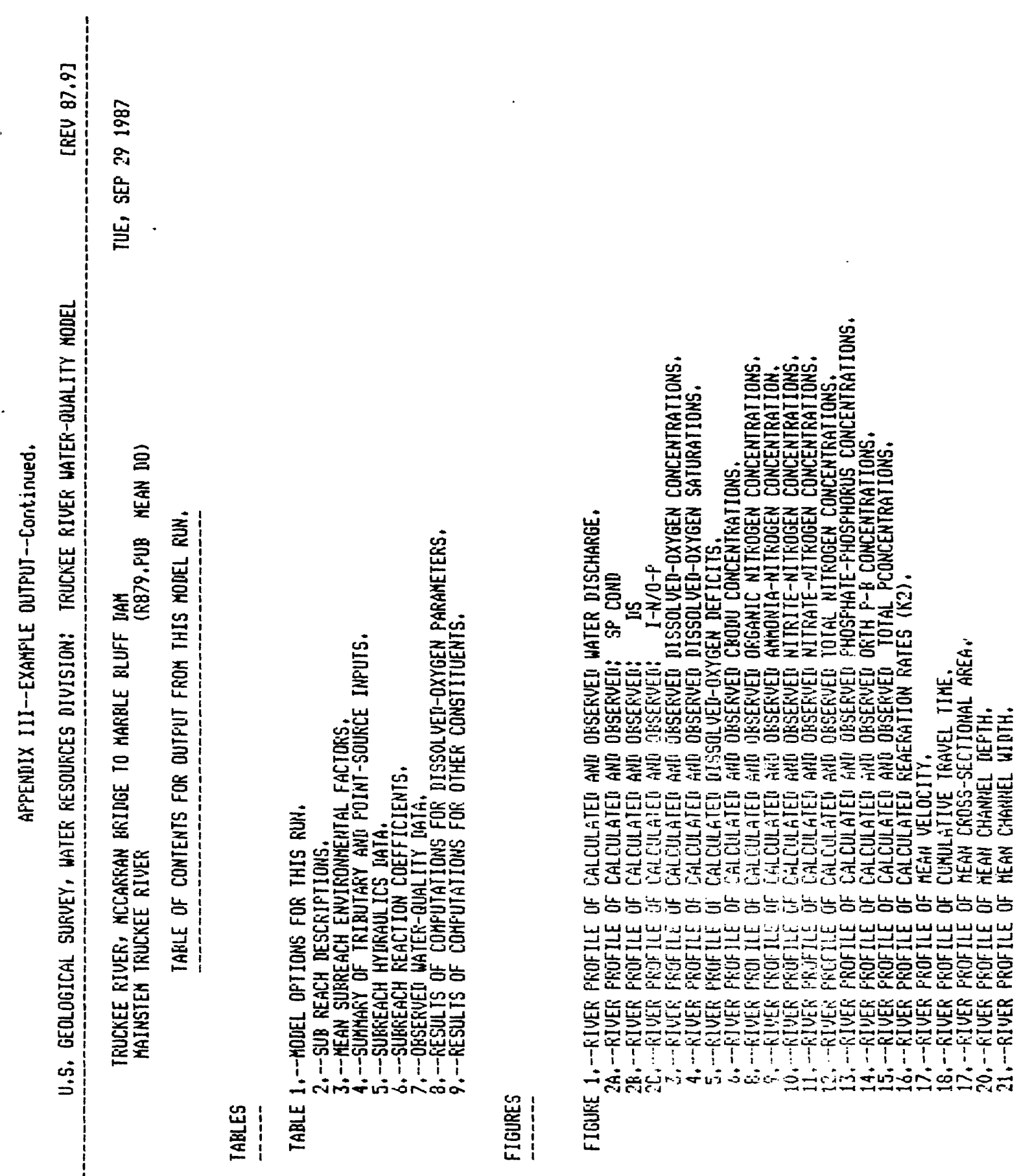




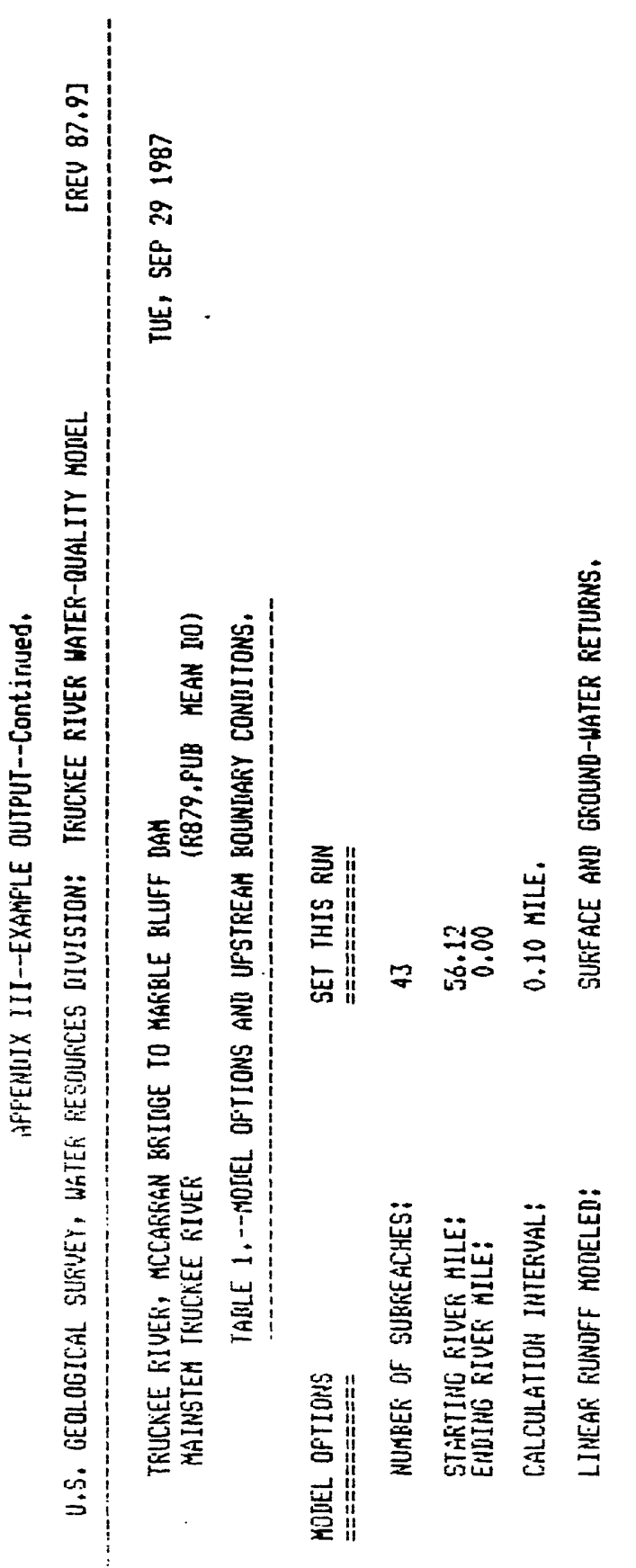




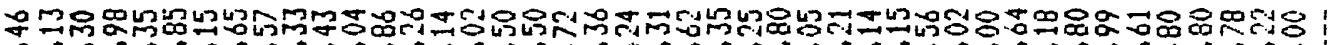

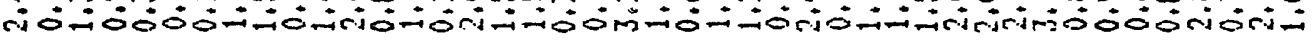

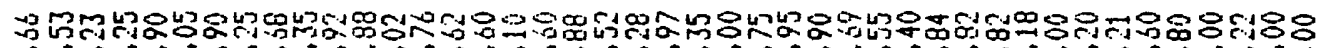
й

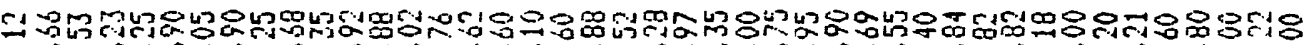

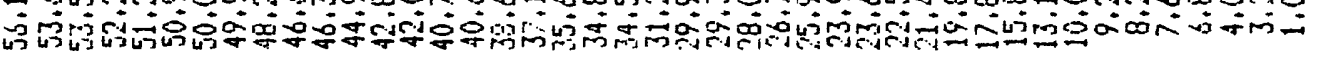

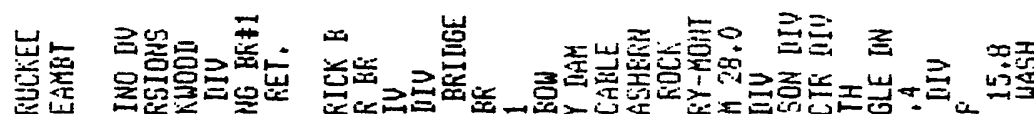

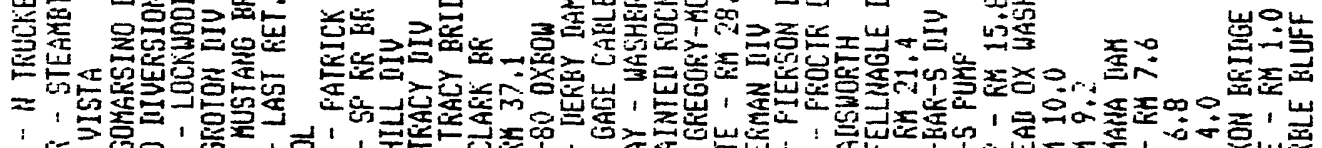

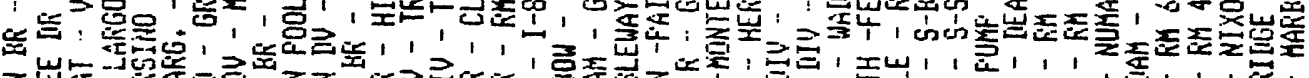

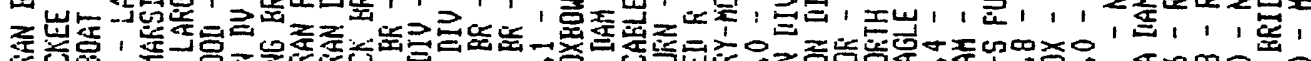

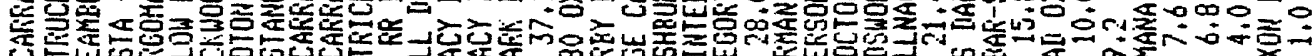

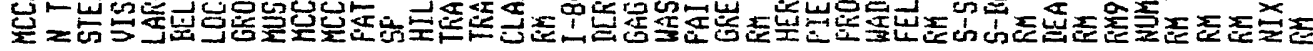

- rum 


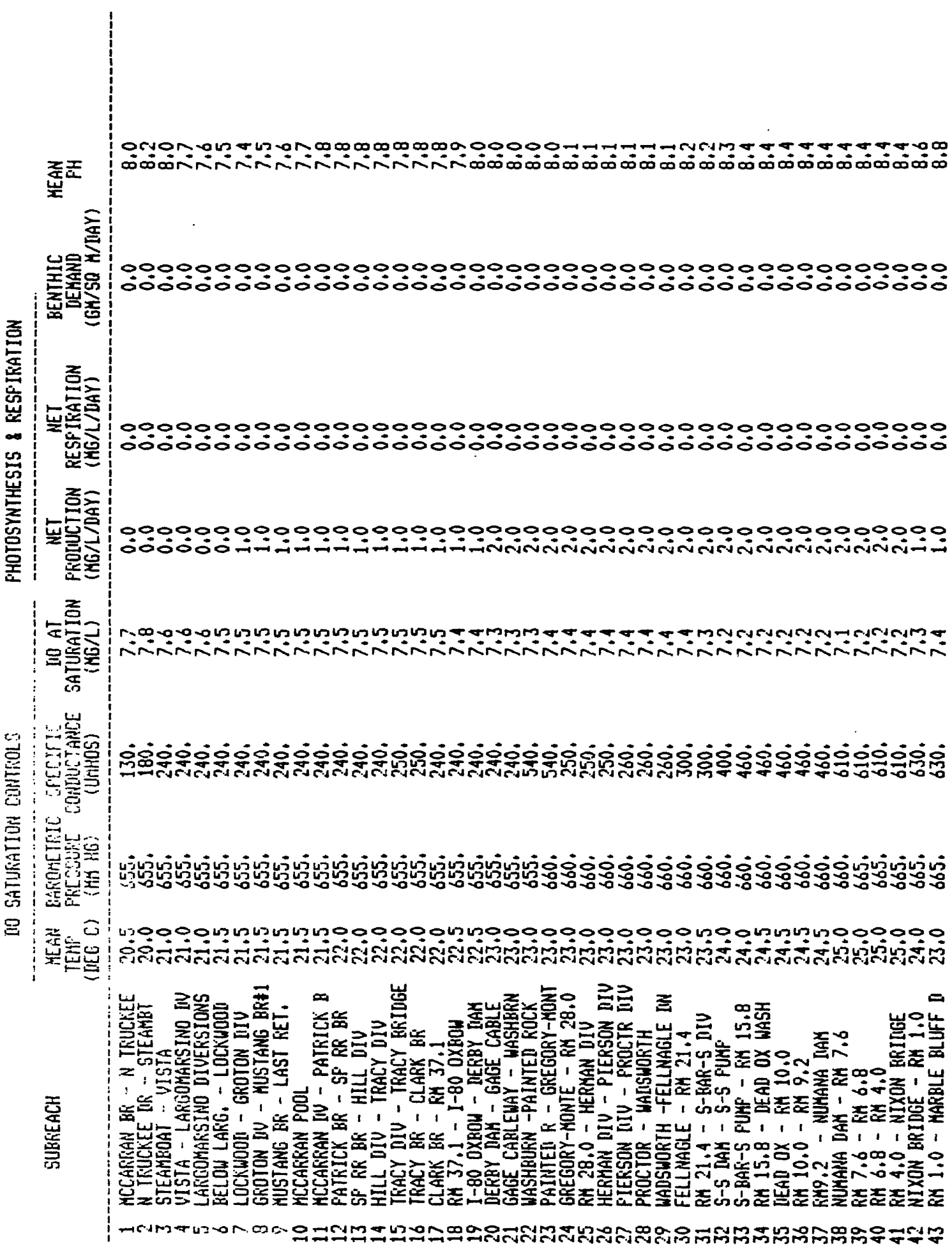




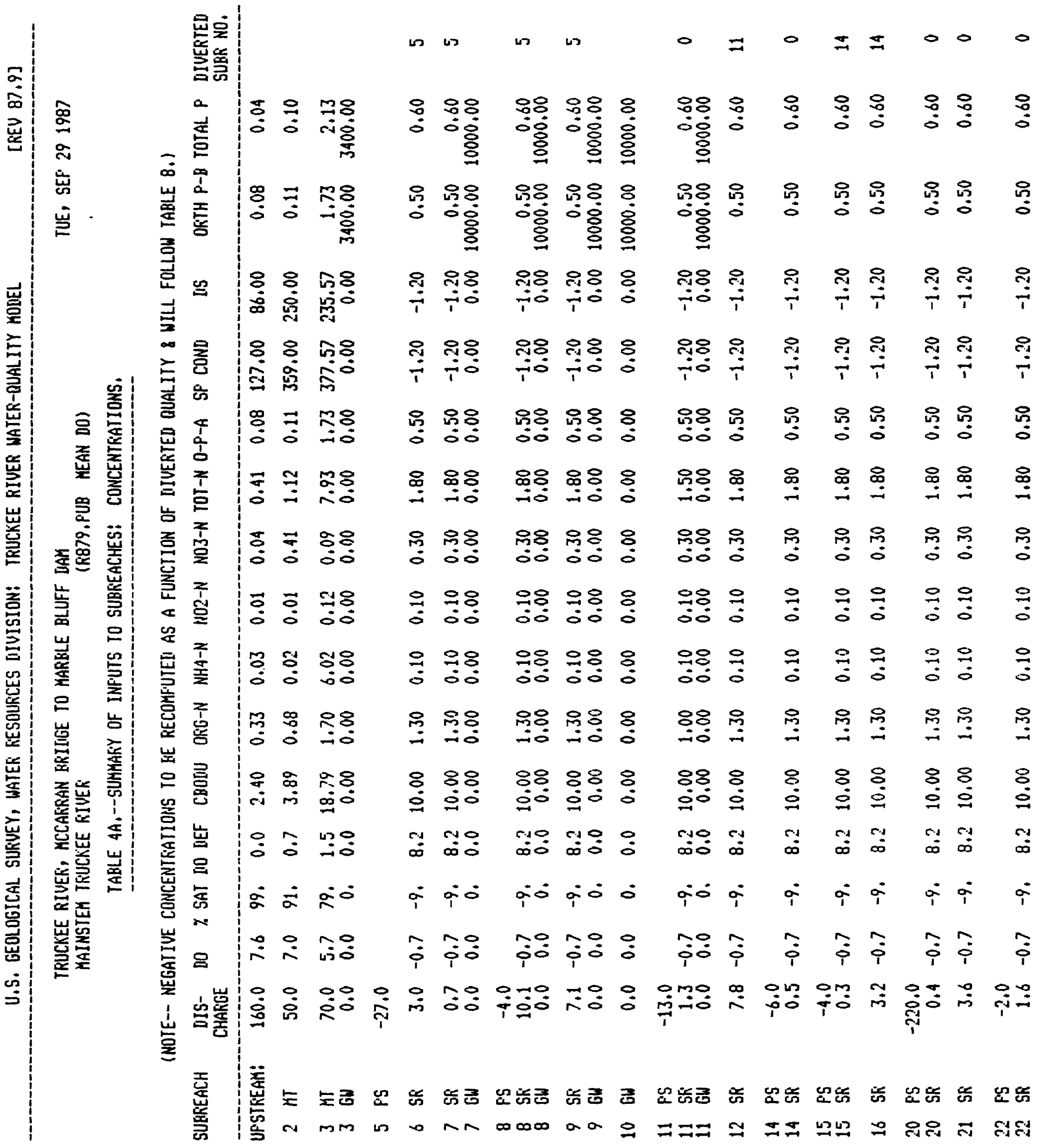




\begin{tabular}{|c|c|c|c|c|c|c|c|c|c|c|c|c|c|c|c|c|c|c|c|}
\hline 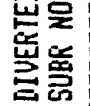 & 0 & 0 & 0 & 0 & 0 & & & 옹 & & 盟 & & & & & & & $\mathbb{p}$ & $\mathbb{m}$ & \\
\hline 站 & $\begin{array}{l}8 \\
0 \\
0\end{array}$ & $\stackrel{8}{\circ}$ & 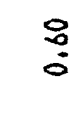 & 号 & $\stackrel{8}{0}$ & $\frac{9}{0}$ & $\frac{9}{0}$ & 造晋 & $\begin{array}{l}80 \\
00\end{array}$ & 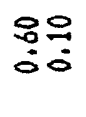 & $\frac{9}{0}$ & $\frac{9}{0}$ & $\stackrel{9}{0}$ & $\because$ & $\dddot{9}$ & $\frac{0}{0}$ & $\begin{array}{l}80 \\
00 \\
00\end{array}$ & $\begin{array}{l}80 \\
80\end{array}$ & $\frac{9}{0}$ \\
\hline 㤩 & ?ำ & 号 & "3 & 量 & 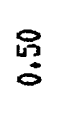 & $\frac{9}{0}$ & $\frac{9}{0}$ & 웅음 & 용응 & 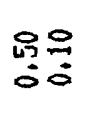 & $\frac{9}{0}$ & $\fallingdotseq$ & $\frac{9}{0}$ & $\dddot{7}$ & $\frac{9}{0}$ & $\frac{0}{0}$ & 몽응 & 용응 & $\frac{0}{0}$ \\
\hline 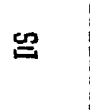 & $\stackrel{\text { ণิ }}{i}$ & $\stackrel{\overbrace{}}{\stackrel{7}{*}}$ & $\stackrel{\overbrace{}}{\stackrel{7}{\dagger}}$ & 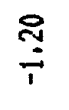 & $\stackrel{\overbrace{}}{\stackrel{1}{\dagger}}$ & $\begin{array}{l}8 \\
8 \\
\text { 옹 }\end{array}$ & 号 & 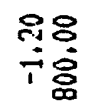 & $\begin{array}{l}980 \\
\div 8 \\
18\end{array}$ & $\begin{array}{l}908 \\
\div 8 \\
18\end{array}$ & $\begin{array}{l}8 \\
8 \\
8\end{array}$ & $\begin{array}{l}8 \\
0 \\
0 \\
0\end{array}$ & ¿ & $\begin{array}{l}8 \\
\vdots \\
\\
0\end{array}$ & $\begin{array}{l}8 \\
\stackrel{\circ}{\circ} \\
\text { 品 }\end{array}$ & 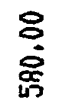 & 옹유 & 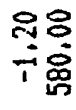 & $\begin{array}{l}8 \\
\text { 怘 }\end{array}$ \\
\hline 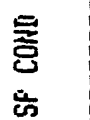 & $\stackrel{\overbrace{}}{\stackrel{i}{1}}$ & i̊ & $\stackrel{\overbrace{}}{\stackrel{+}{1}}$ & $\stackrel{9}{\stackrel{9}{-}}$ & 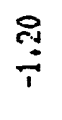 & $\begin{array}{l}8 \\
\vdots \\
\dot{0}\end{array}$ & 웅 & 옹ㅇㅇ & 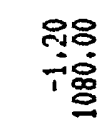 & 용 & $\begin{array}{l}8 \\
\stackrel{8}{\circ} \\
\stackrel{8}{\circ}\end{array}$ & $\begin{array}{l}8 \\
8 \\
0 \\
0 \\
0\end{array}$ & 吕 & 号 & $\begin{array}{l}8 \\
\stackrel{8}{\circ} \\
\text { 足 }\end{array}$ & $\begin{array}{l}8 \\
\stackrel{8}{\circ} \\
\dot{8}\end{array}$ & 욤유 & $\begin{array}{l}\text { î० } \\
\text { 18 }\end{array}$ & 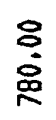 \\
\hline$\frac{a}{4}$ & ถึ & 品 & 号 & 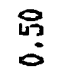 & 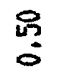 & $\frac{9}{0}$ & $\frac{0}{0}$ & 용응 & 용으 & 옹유 & $\frac{9}{0}$ & $\frac{9}{0}$ & $\frac{9}{0}$ & $\frac{9}{0}$ & $\frac{9}{0}$ & $\frac{0}{0}$ & 옹응 & 융ㅇํ & $\stackrel{0}{0}$ \\
\hline 预 & $\stackrel{8}{8}$ & $\stackrel{8}{=}$ & $\stackrel{8}{\stackrel{8}{二}}$ & $\stackrel{\$ 口}{=}$ & $\stackrel{8}{\square}$ & $\stackrel{8}{=}$ & $\stackrel{8}{\$ 口}$ & 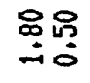 & 骂员 & 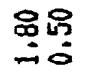 & ㅇํㅇ & $\frac{9}{0}$ & $\frac{9}{0}$ & $\frac{9}{0}$ & $\stackrel{8}{-1}$ & $\stackrel{8}{=}$ & 吕昌 & 吕。 & $\stackrel{8}{9}$ \\
\hline 定 & ? & ? & 蛋 & ? & ?ำ & $\stackrel{8}{=}$ & $\stackrel{\$}{=}$ & 용영 & 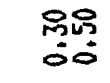 & 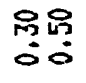 & 웅 & $\frac{0}{0}$ & $\frac{9}{0}$ & $\frac{9}{0}$ & $\stackrel{8}{-1}$ & $\stackrel{8}{=}$ & 용용 & 융영 & $\stackrel{8}{=}$ \\
\hline 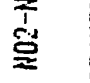 & $\frac{0}{0}$ & $\frac{9}{0}$ & $\stackrel{9}{0}$ & $\frac{1}{0}$ & $\frac{O}{0}$ & 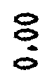 & $\stackrel{8}{8}$ & 웅영 & $\begin{array}{l}98 \\
50\end{array}$ & 용ㅇㅇ & $\stackrel{8}{8}$ & 8 & $\begin{array}{l}8 \\
0\end{array}$ & $\begin{array}{l}8 \\
0\end{array}$ & $\begin{array}{l}8 \\
0\end{array}$ & $\frac{8}{0}$ & $\begin{array}{l}90 \\
50\end{array}$ & $\frac{98}{60}$ & $\begin{array}{l}8 \\
0\end{array}$ \\
\hline 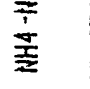 & $\frac{0}{0}$ & $\frac{9}{0}$ & $\frac{9}{0}$ & $\frac{0}{0}$ & $\frac{0}{0}$ & $\begin{array}{l}8 \\
0 \\
0\end{array}$ & $\frac{8}{0}$ & $\because 8$ & $\begin{array}{l}\frac{98}{08} \\
00\end{array}$ & $\begin{array}{l}098 \\
00\end{array}$ & $\begin{array}{l}8 \\
\vdots\end{array}$ & $\stackrel{8}{0}$ & $\begin{array}{l}8 \\
0\end{array}$ & $\stackrel{8}{8}$ & $\begin{array}{l}8 \\
0\end{array}$ & $\frac{8}{0}$ & $\frac{98}{0}$ & $\begin{array}{l}58 \\
08\end{array}$ & 3 \\
\hline 勇 & $\stackrel{?}{?}$ & 官 & 点 & 足 & 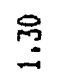 & $\begin{array}{l}8 \\
0 \\
0\end{array}$ & 8 & 용ㅇㅇ & 용 & $\begin{array}{l}\text { 官8 } \\
-10\end{array}$ & $\stackrel{8}{8}$ & $\stackrel{8}{\circ}$ & $\begin{array}{l}8 \\
0\end{array}$ & $\frac{8}{8}$ & $\stackrel{8}{0}$ & $\begin{array}{l}8 \\
0\end{array}$ & $\begin{array}{l}\text { 용ㅇㅇ } \\
-10\end{array}$ & 용ㅇㅇ & 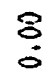 \\
\hline 忌 & $\begin{array}{l}8 \\
0 \\
0\end{array}$ & $\begin{array}{l}8 \\
0 \\
0\end{array}$ & $\begin{array}{l}8 \\
0 \\
0\end{array}$ & $\begin{array}{l}8 \\
0 \\
0\end{array}$ & $\begin{array}{l}8 \\
0 \\
0\end{array}$ & $\stackrel{8}{1}$ & $\stackrel{8}{\circ}$ & $\begin{array}{l}88 \\
40 \\
40\end{array}$ & 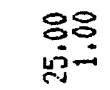 & $\begin{array}{l}88 \\
430 \\
43\end{array}$ & $\stackrel{8}{=}$ & $\stackrel{8}{-}$ & $\stackrel{8}{\circ}$ & $\stackrel{8}{-1}$ & $\stackrel{8}{\circ}$ & $\stackrel{8}{-}$ & $\begin{array}{l}88 \\
0 \\
0\end{array}$ & $\begin{array}{l}88 \\
0-1 \\
0-1\end{array}$ & 8 \\
\hline 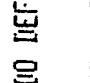 & 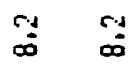 & $\underset{\infty}{\infty}$ & @ & $\begin{array}{l}n \\
0\end{array}$ & $\begin{array}{c}n \\
\infty \\
\infty\end{array}$ & $\stackrel{\infty}{n}$ & $\stackrel{\infty}{n}$ & 車象 & in & 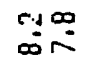 & $\stackrel{\mathscr{O}}{\sim}$ & $\stackrel{\infty}{\sim}$ & $\stackrel{\infty}{r}$ & $\stackrel{\infty}{\pi}$ & $\stackrel{0}{n}$ & 2 & $a a$ & 20 & $\ddot{n}$ \\
\hline 㤐 & i & $a_{i}^{\circ}$ & i & $\dot{i}^{*}$ & $a_{i}^{0}$ & $\dot{0}$ & $\dot{0}$ & i் & $\ddot{i}$ & $a_{i}^{*} \dot{a}^{-}$ & 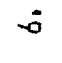 & 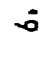 & $\dot{0}$ & $\therefore$ & ") & $\dot{0}$ & $a^{\circ}$ & $a^{\circ}$ & $\dot{\sigma}^{\circ}$ \\
\hline Э & $\hat{i}$ & $\hat{i}$ & $\hat{i}$ & $\tilde{i}$ & $\hat{i}$ & $?$ & 0 & 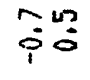 & & & 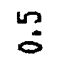 & wa & 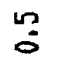 & $?$ & $\stackrel{2}{0}$ & 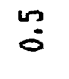 & 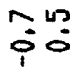 & & ?2 \\
\hline 岕寕 & 亏ั & $\stackrel{\infty}{=}$ & $\frac{0}{\frac{9}{i}}$ & in & un & $\stackrel{\infty}{\sigma}$ & $\frac{0}{9}$ & $\exists$ & $\frac{900}{100}$ & 30 & $\stackrel{\sigma}{0}$ & $\hat{0}$ & ? & "艹 & $\stackrel{0}{\frac{0}{1}}$ & $\ddot{1}$ & जio & \pm 0 & $\hat{0}$ \\
\hline 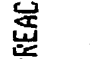 & 总总密 & 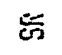 & 起落 & 点 & 妿 & 忐 & 뚕즁 & 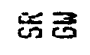 & 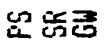 & 孚承 & 쿵 & 쿰 & 쿻 & 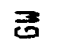 & 류룸 & 룹 & 야⿱류 & 응 궁 & 吾 \\
\hline & 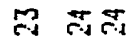 & 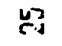 & 急 & $\approx$ & 蜖 & $\stackrel{2}{\approx}$ & 유요 & $\vec{m} \vec{m}$ & SAFA & $m$ & 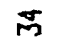 & 点 & 우 & कि & 禺罵 & 点 & 우우 & $\vec{\sigma} \vec{\gamma}$ & $\frac{9}{4}$ \\
\hline
\end{tabular}




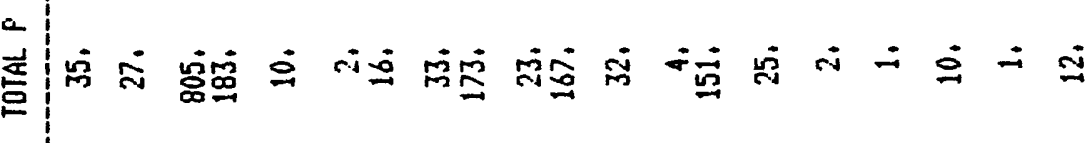

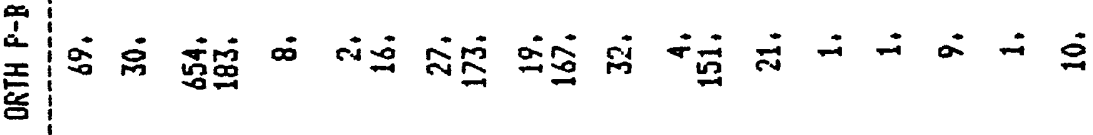

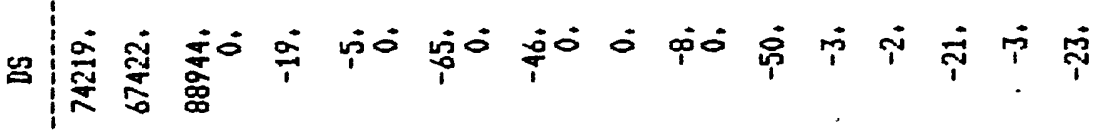

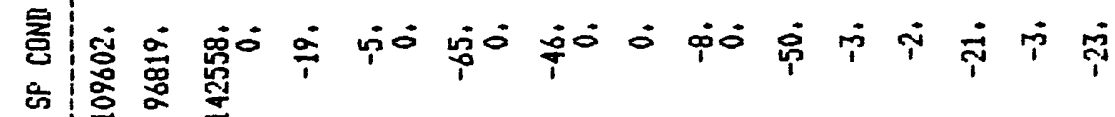
농 영

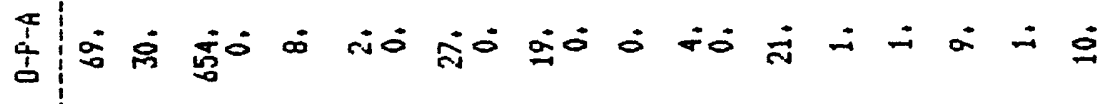

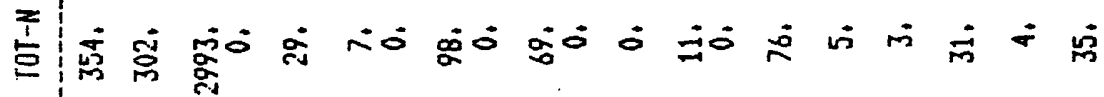

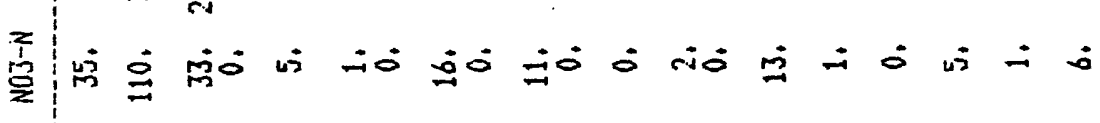
恿 焉

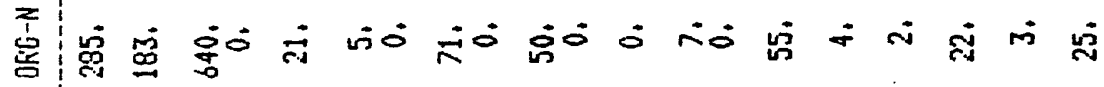

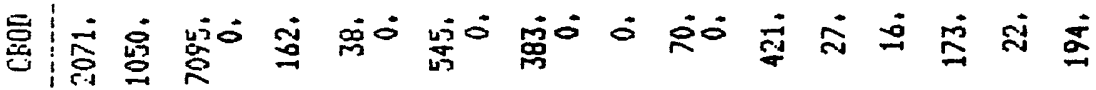

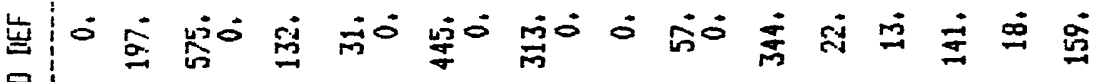
$\Xi$

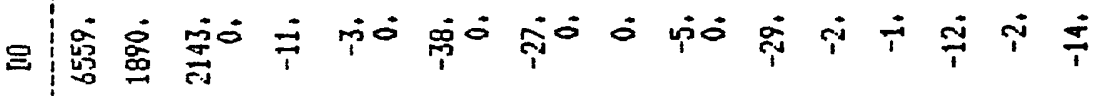

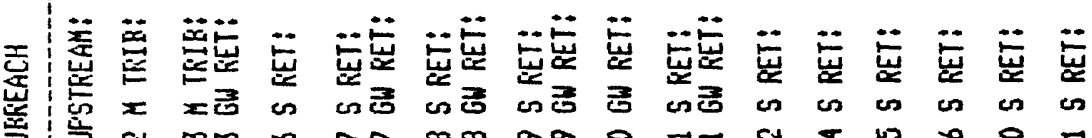


若 $\frac{d}{d} \dot{d}$ is |

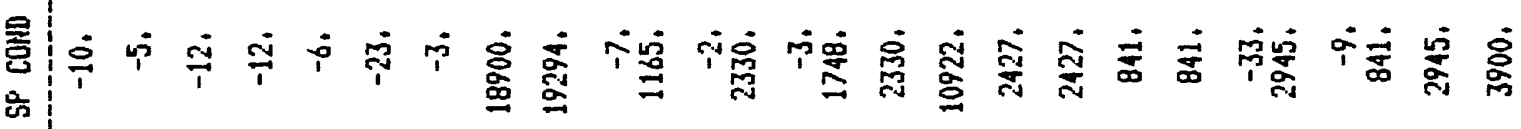
賁

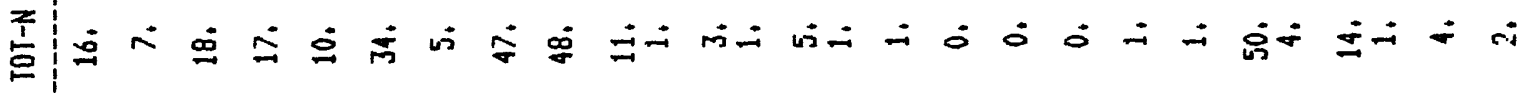
䍗

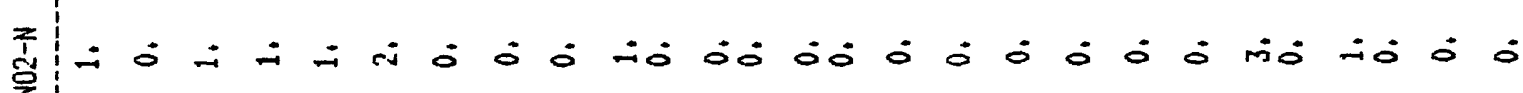

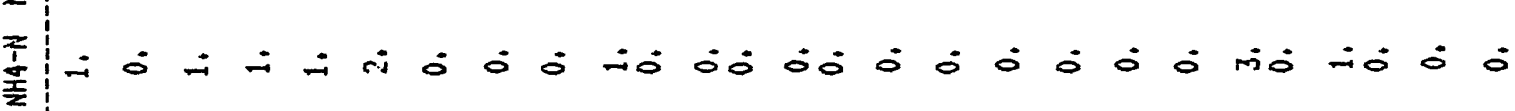
管

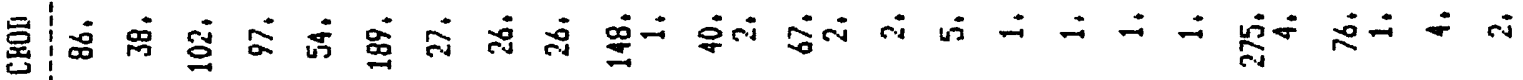

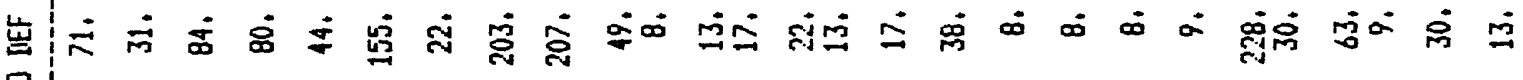

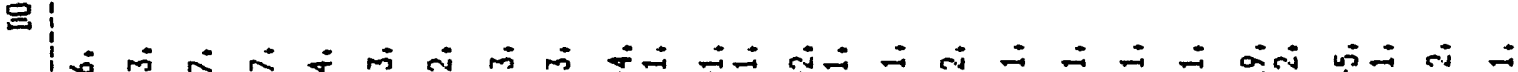

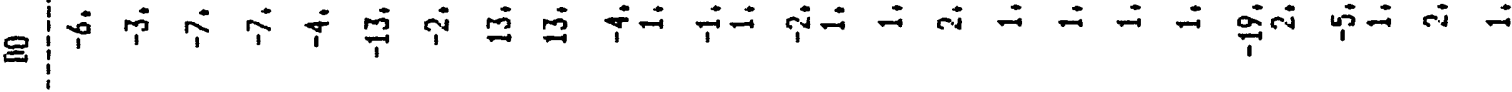

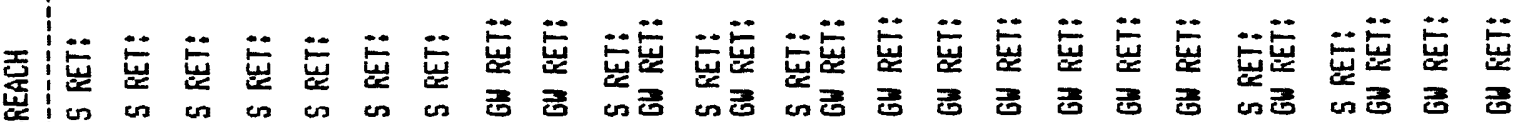

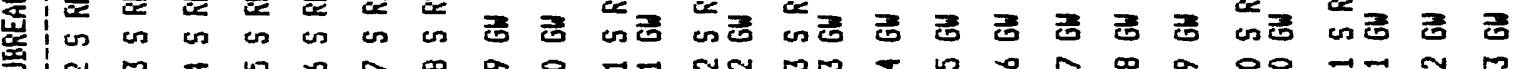

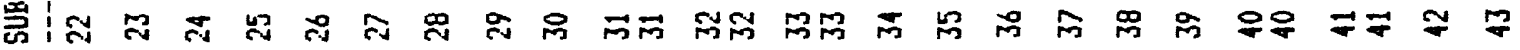


部

咆昜

吉䓌

崖它

岁总

岁岁空

호을

迹行

窝吉

可:

崖

岩

密

䓌突

잉

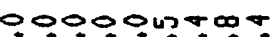

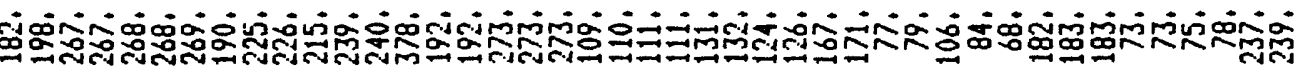

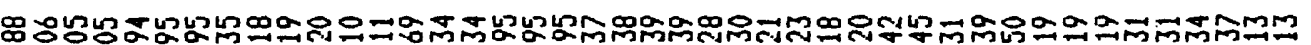

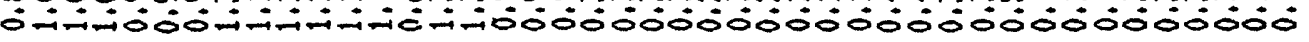

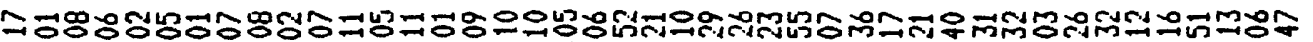
$00000000000000000000000000000000000000000=0$ a

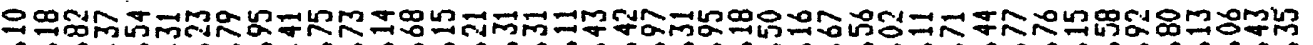

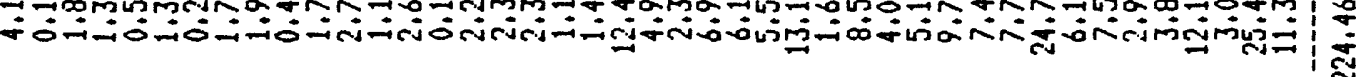

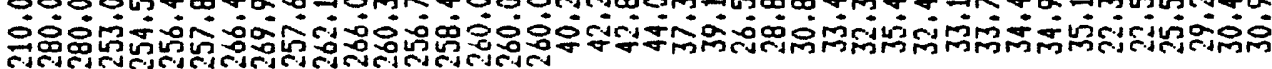

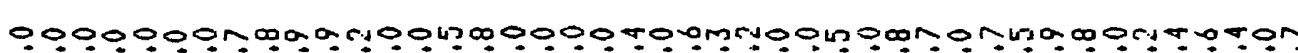

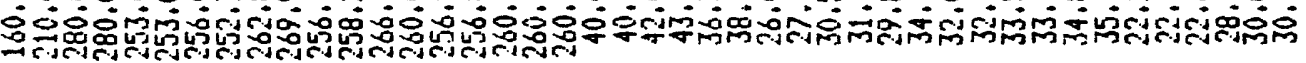

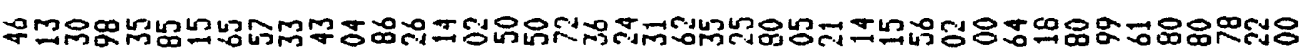

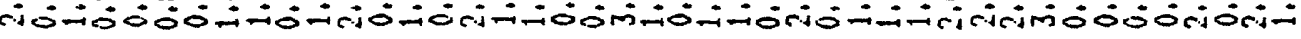

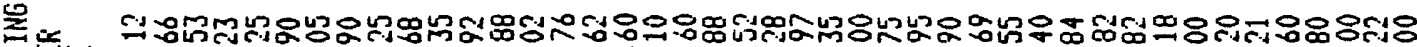
运崖崖 ง

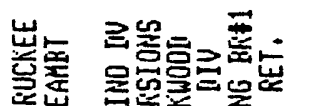

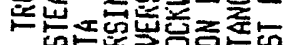

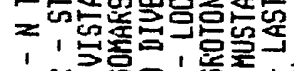

岕岕事

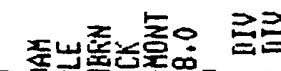

Z

辰

空甹

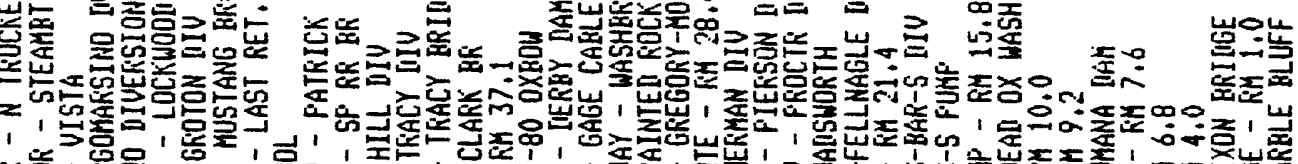

甹

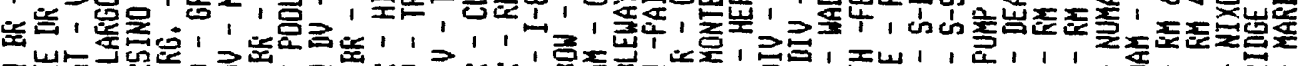

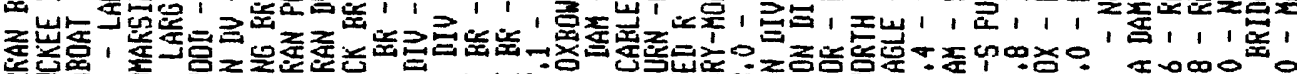

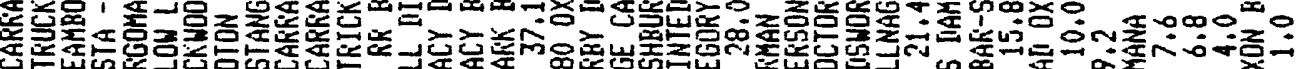

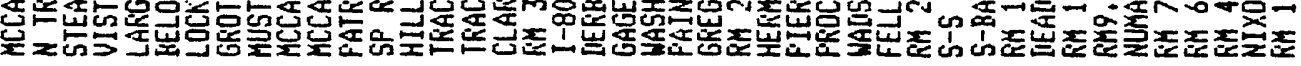

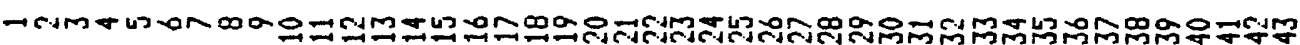




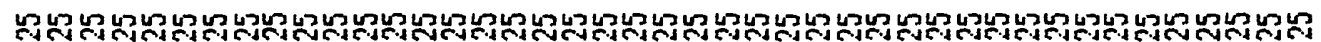
0000000000000000000000000000000000000000000

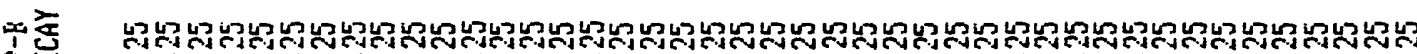
送 000000000000000000000000000000000000000000 䂵

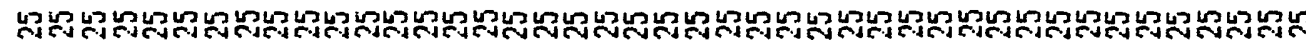
0000000000000000000000000000000000000000000

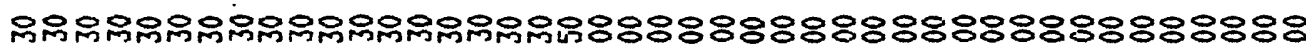

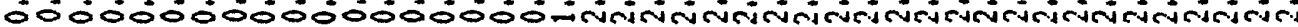
8888888888808888888888888888888888888888888 二

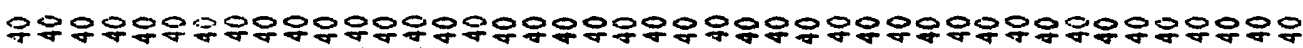

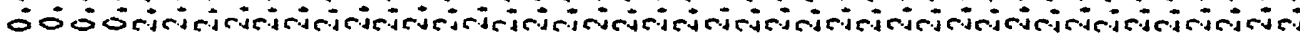

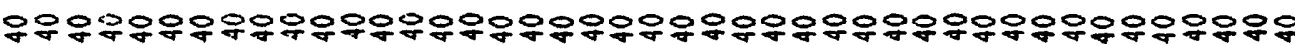

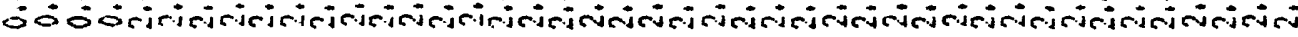

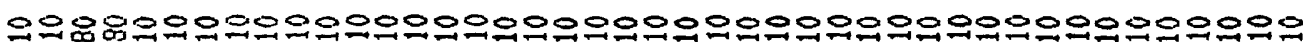
05000000000000000000000000000000000000000

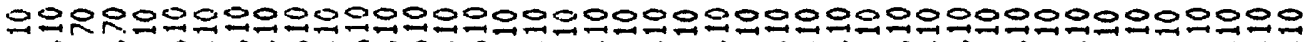

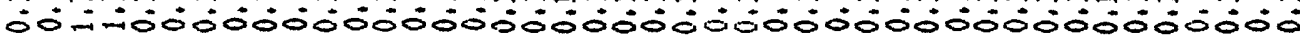

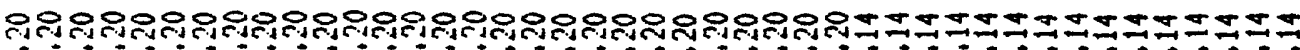

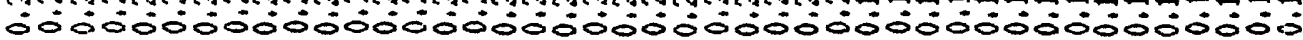

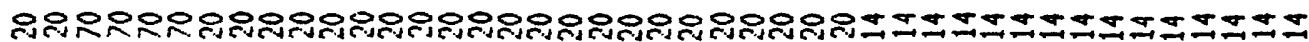

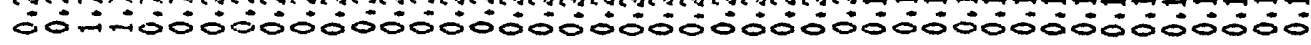
종

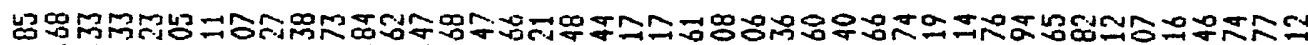

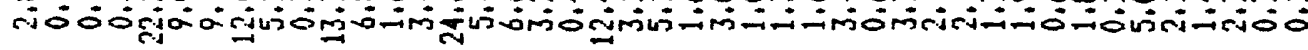




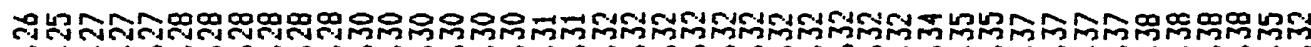
000000000000000000000000000000000000000000

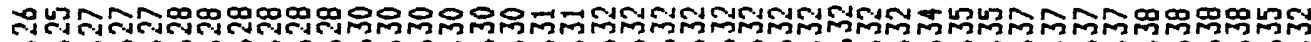
000000000000000000000000000000000000000000

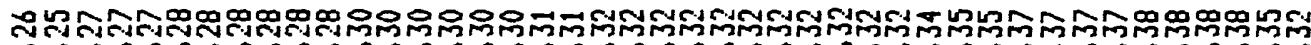
000000000000000000000000000000000000000000

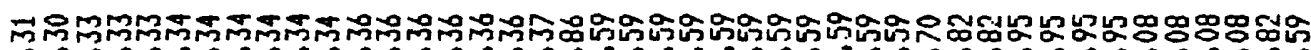
000000000000000000

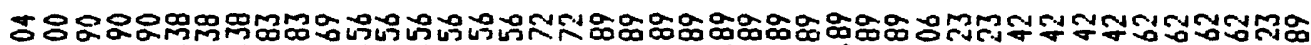

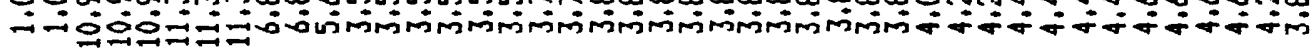

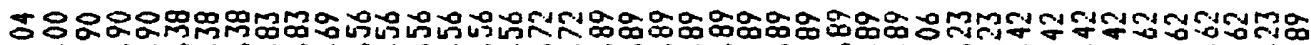

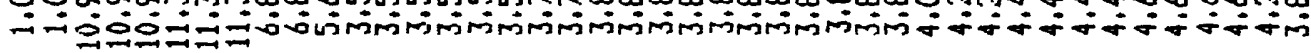

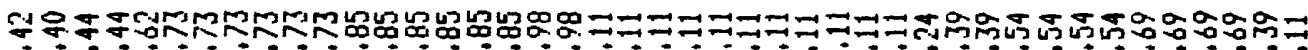

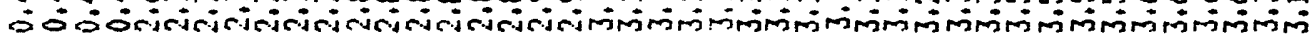

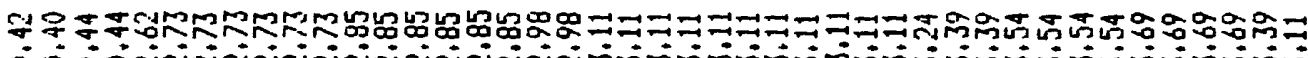

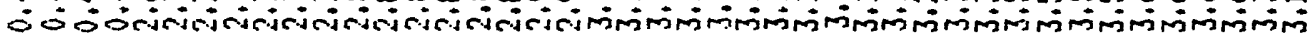

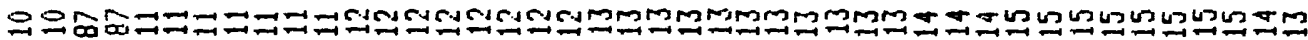

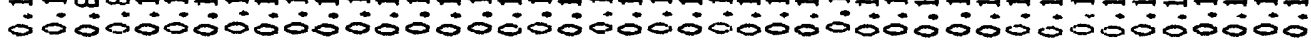

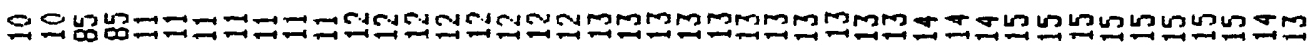
50

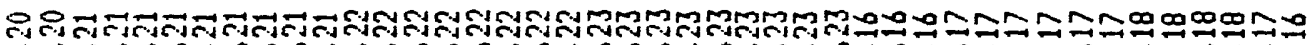
0000000000000000000000000000000000000000000

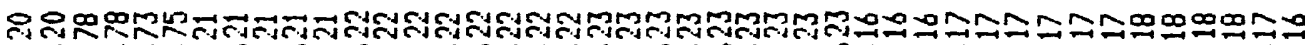
00 1. 000000000000000000000000000000000000000

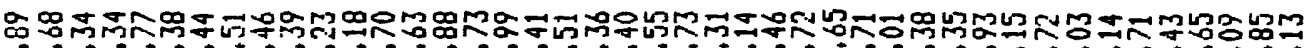

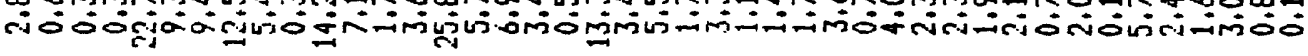

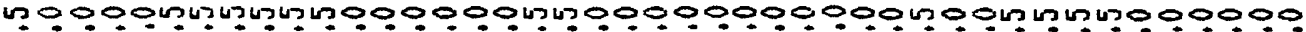

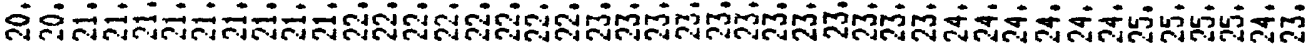

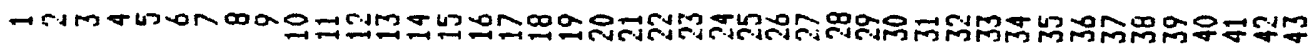




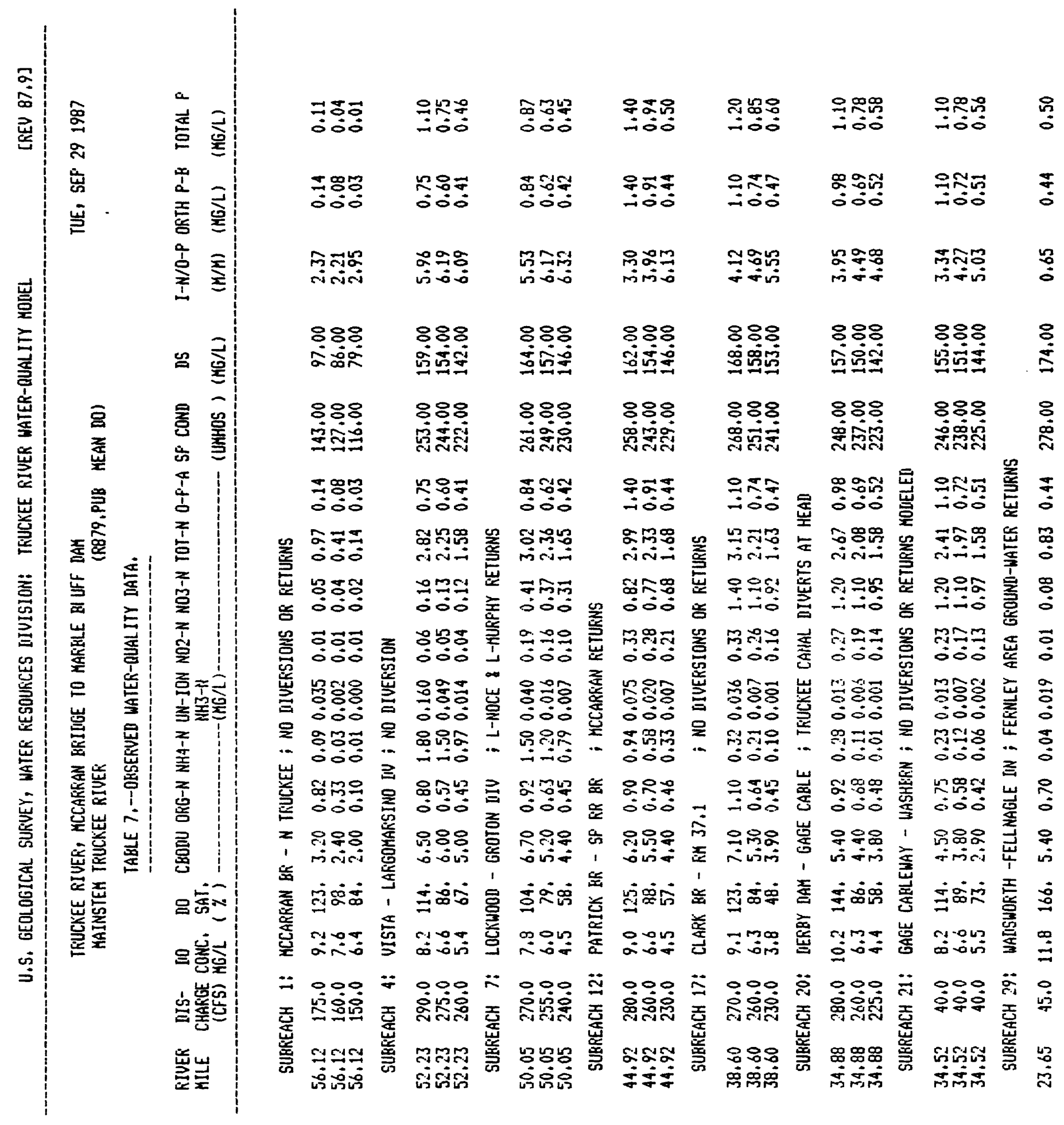




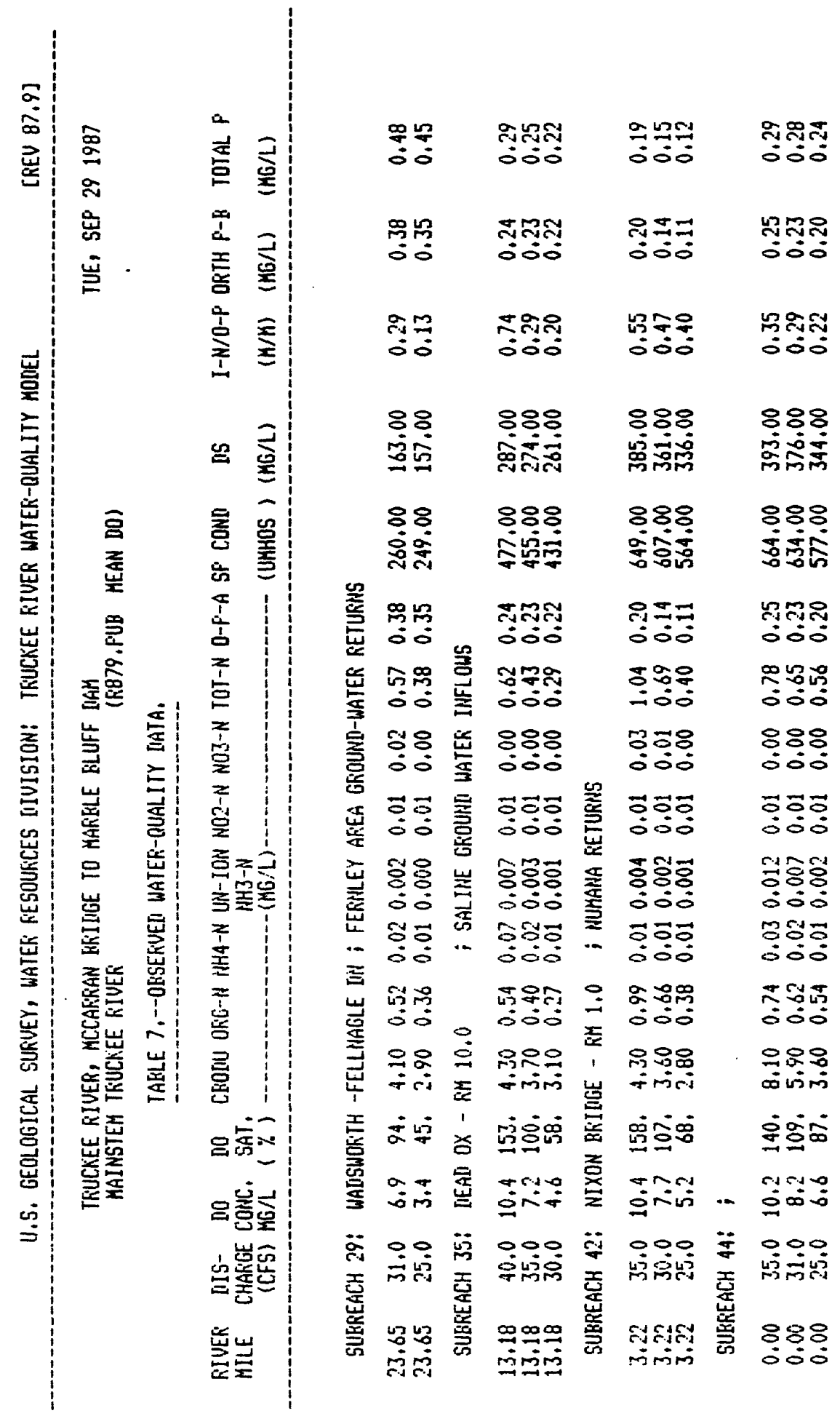




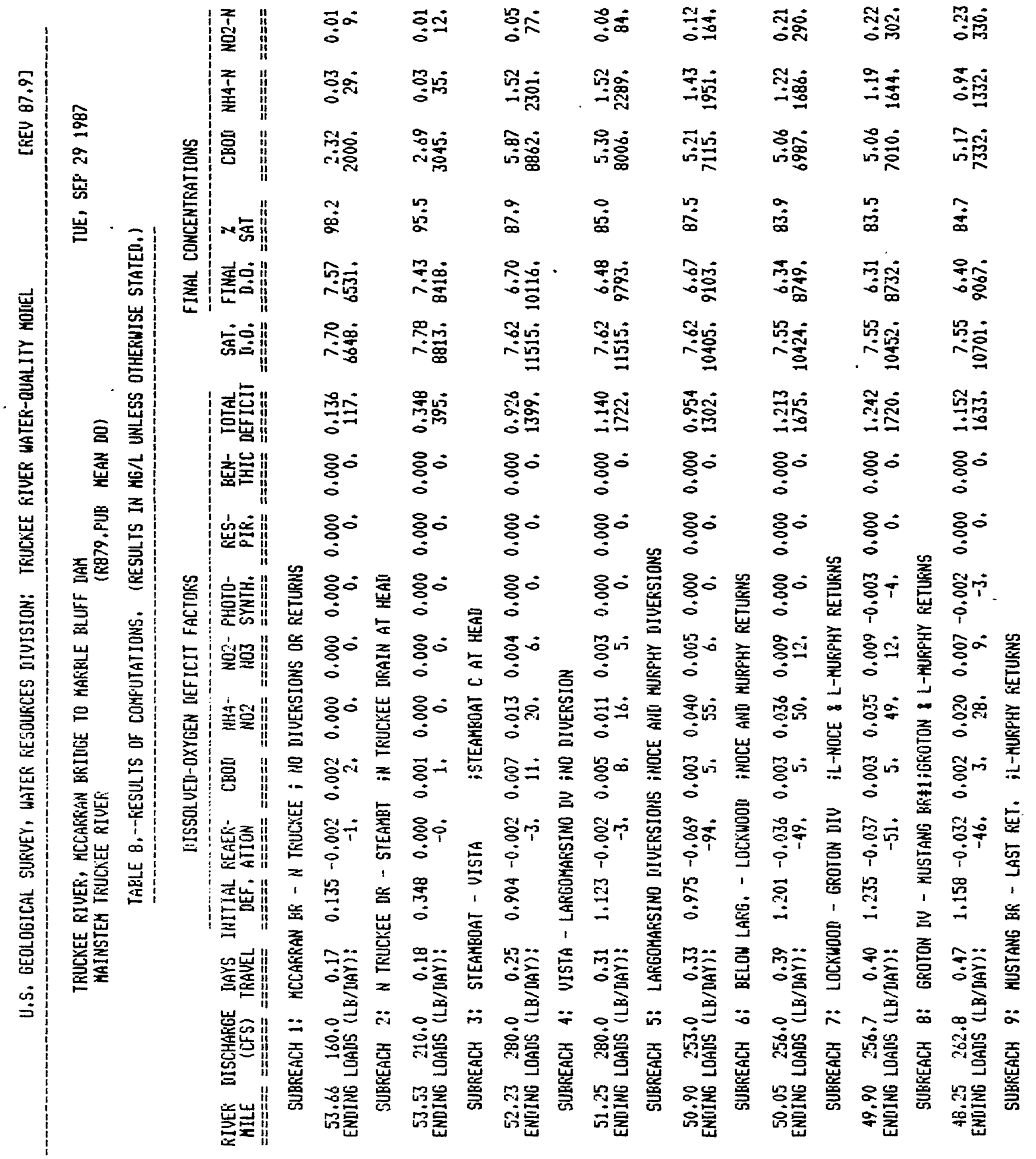




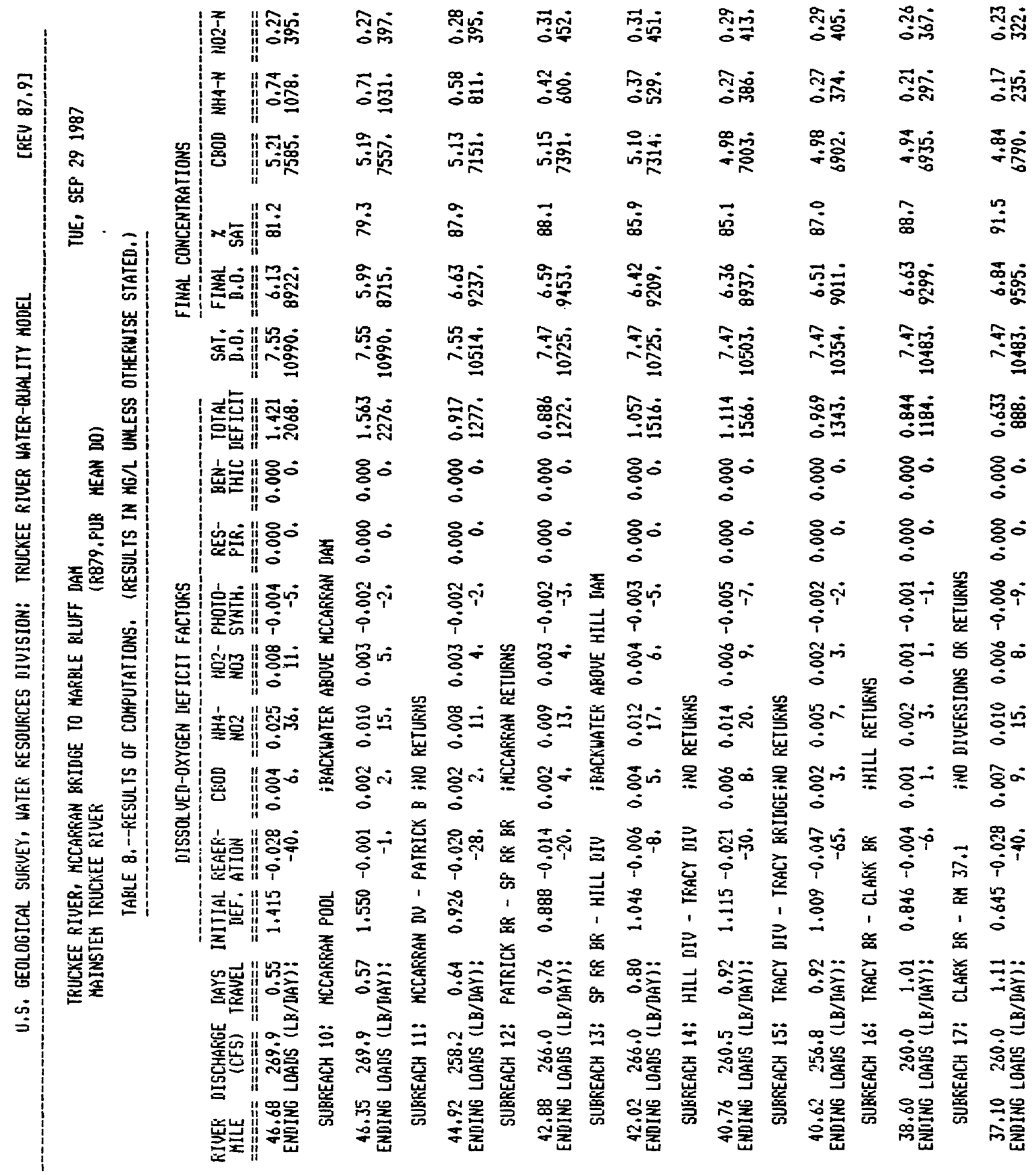




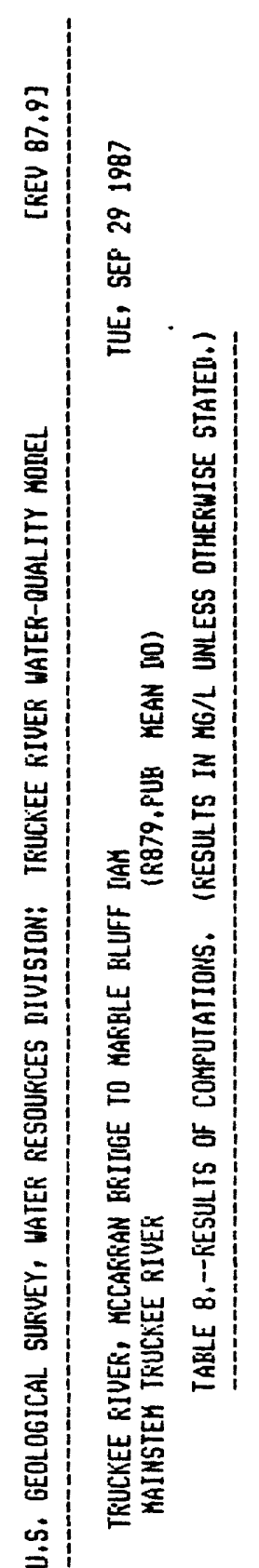

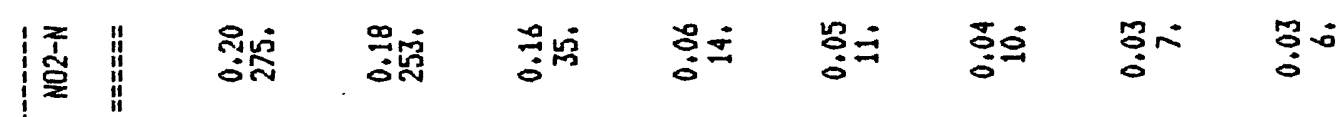

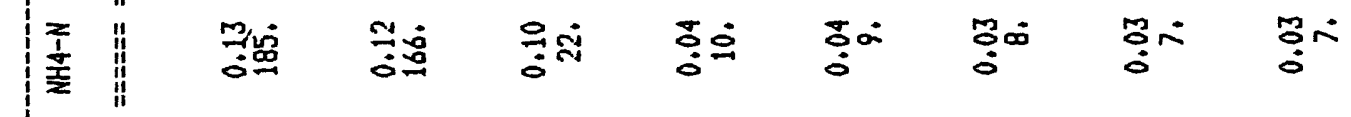
.

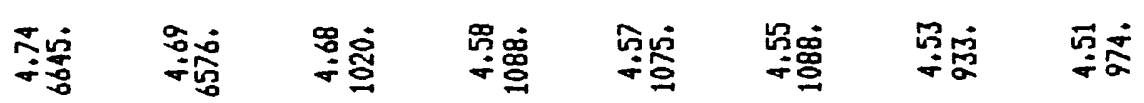
喜 ڤั

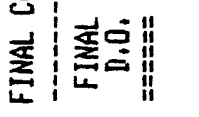
穃

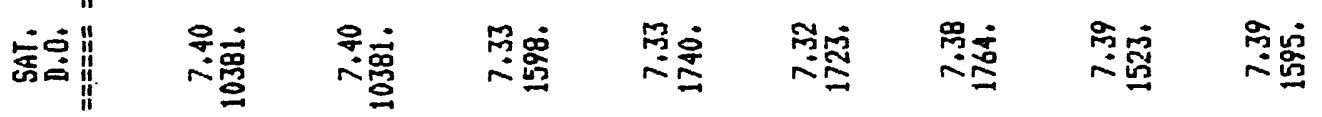

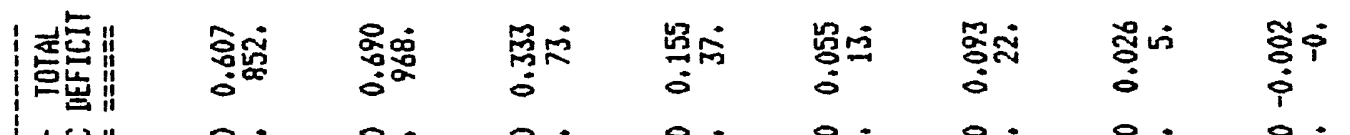

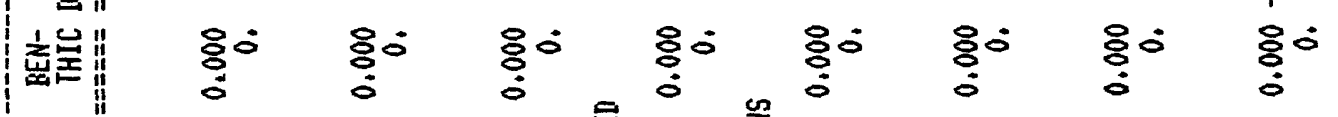

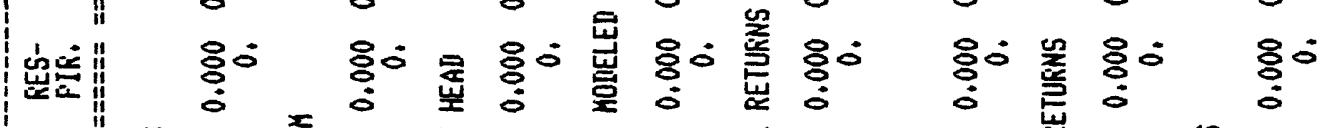

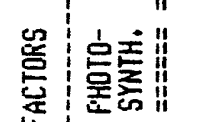

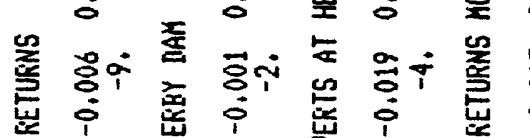
署

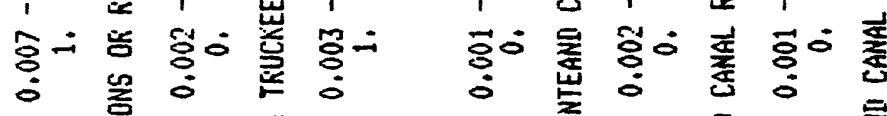




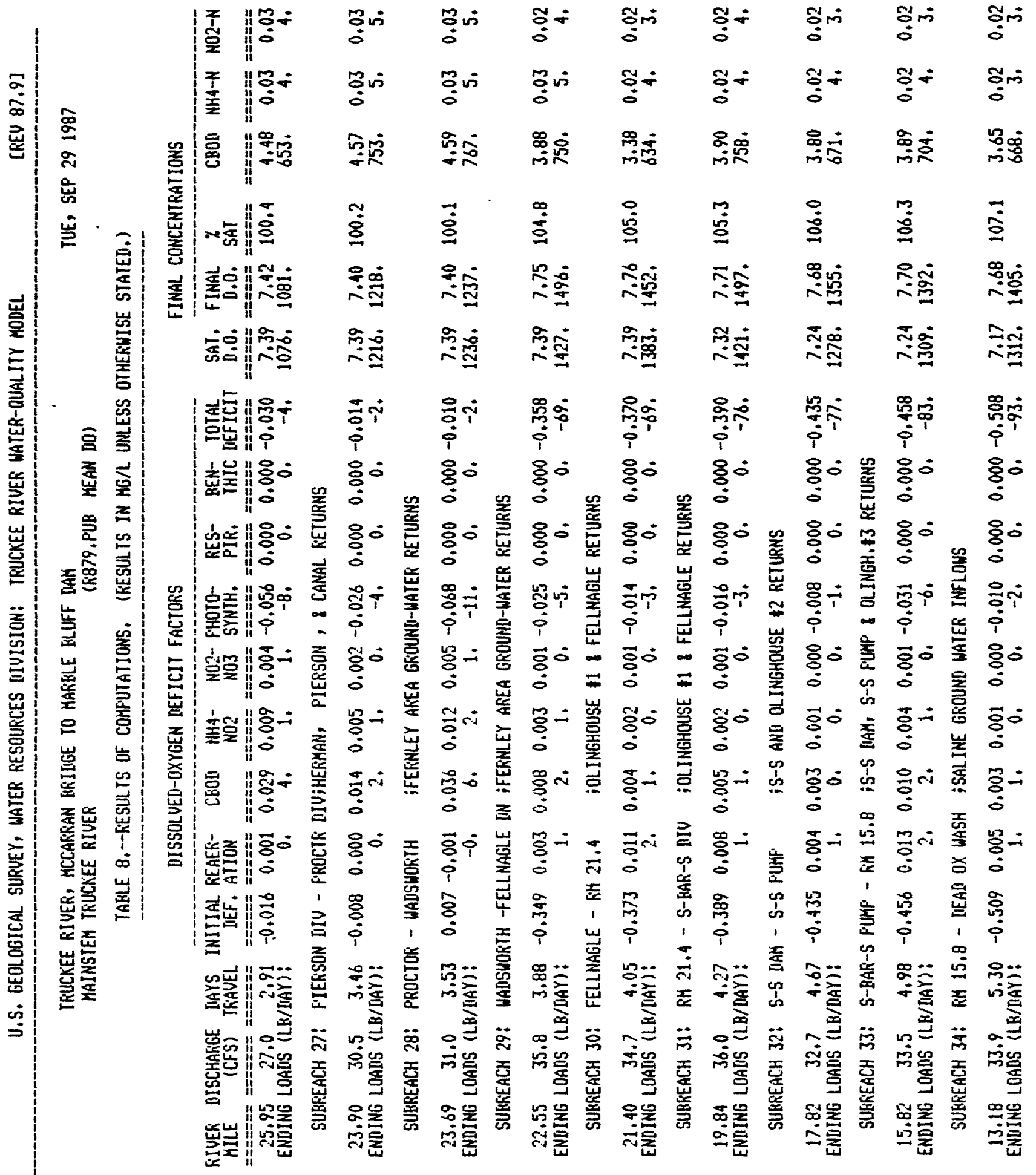




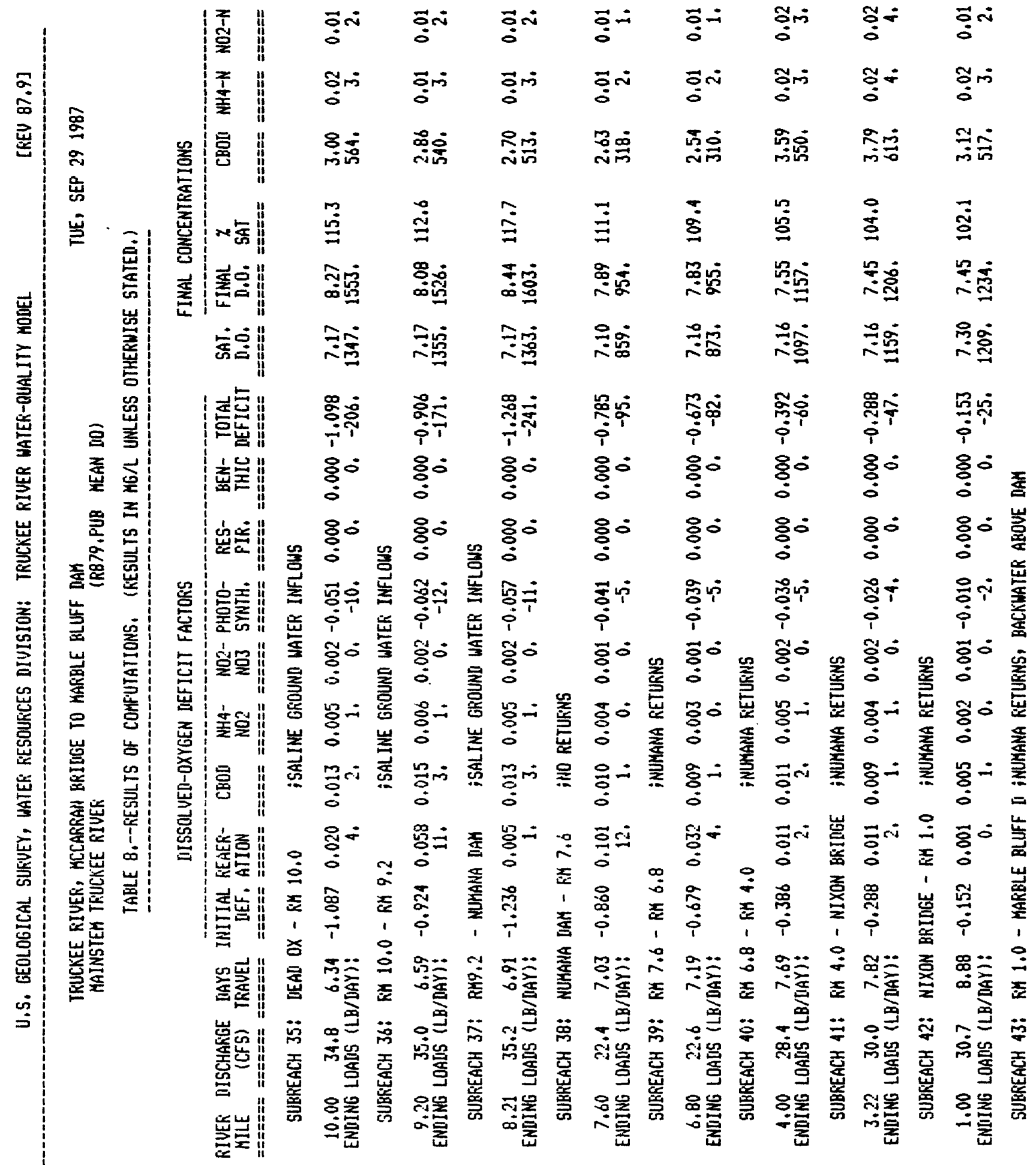




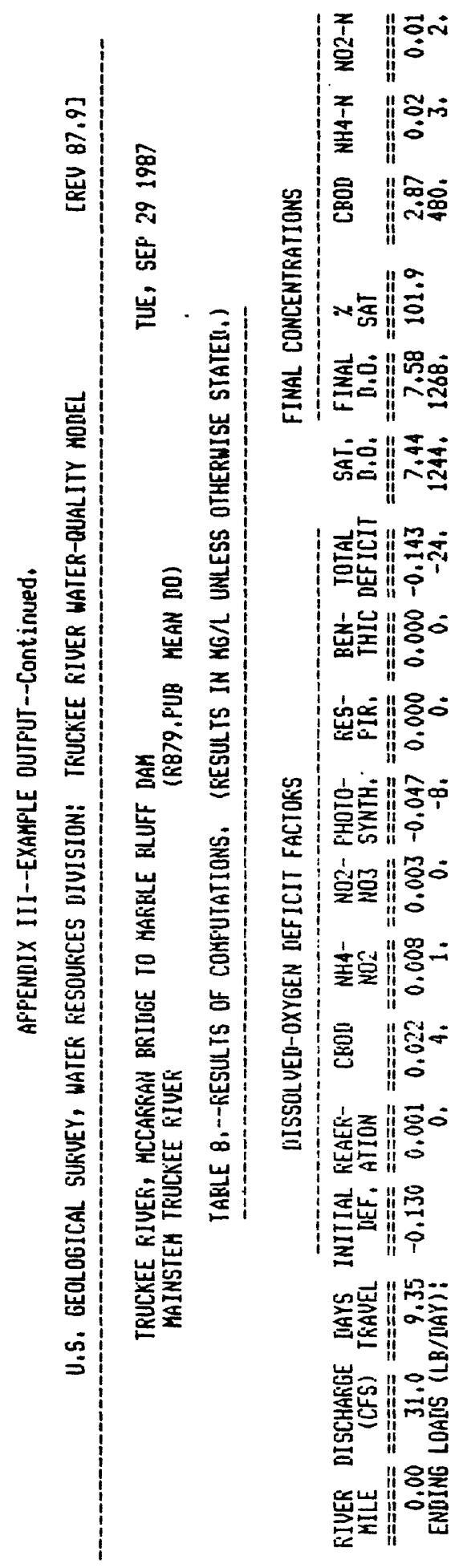




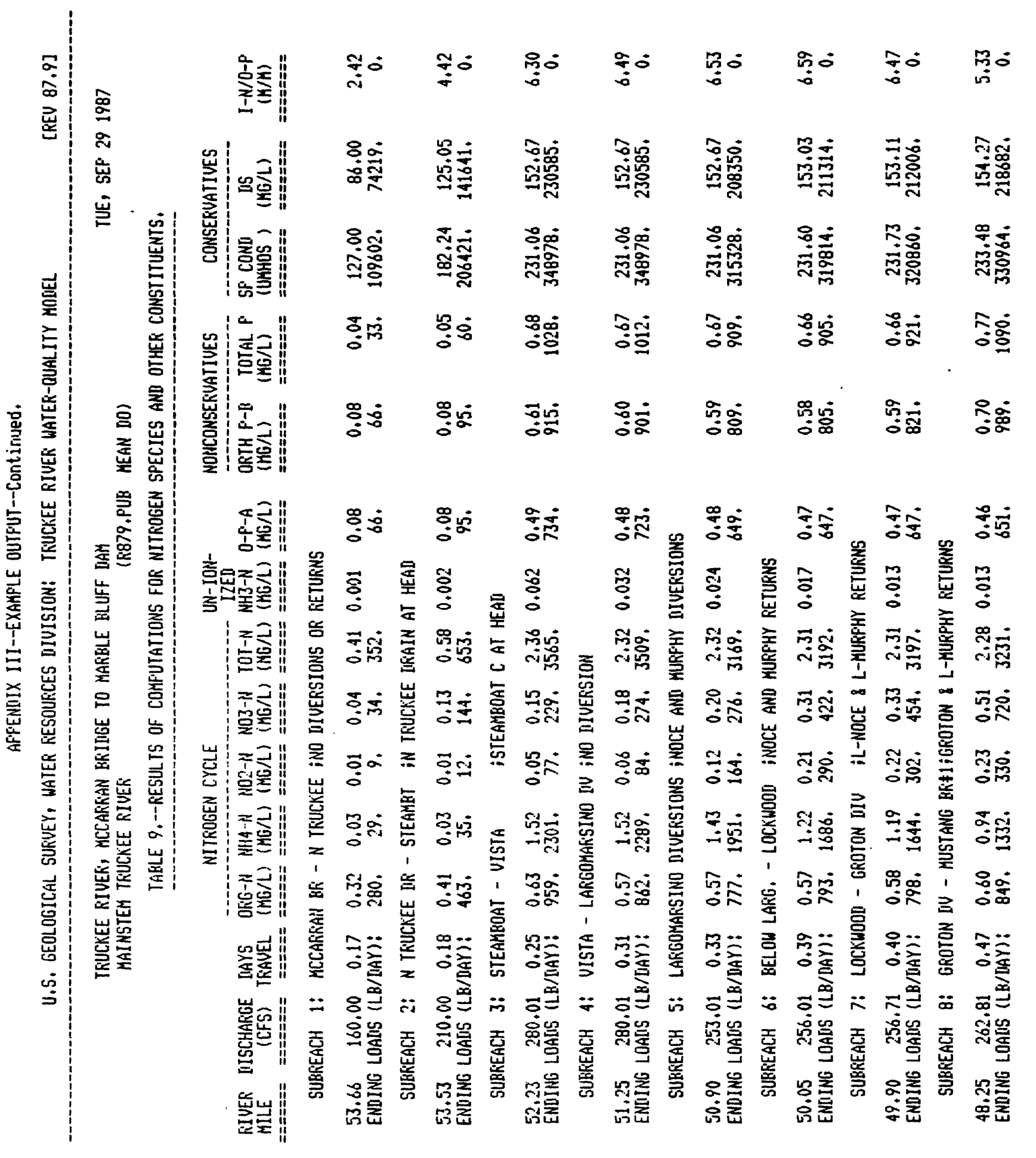




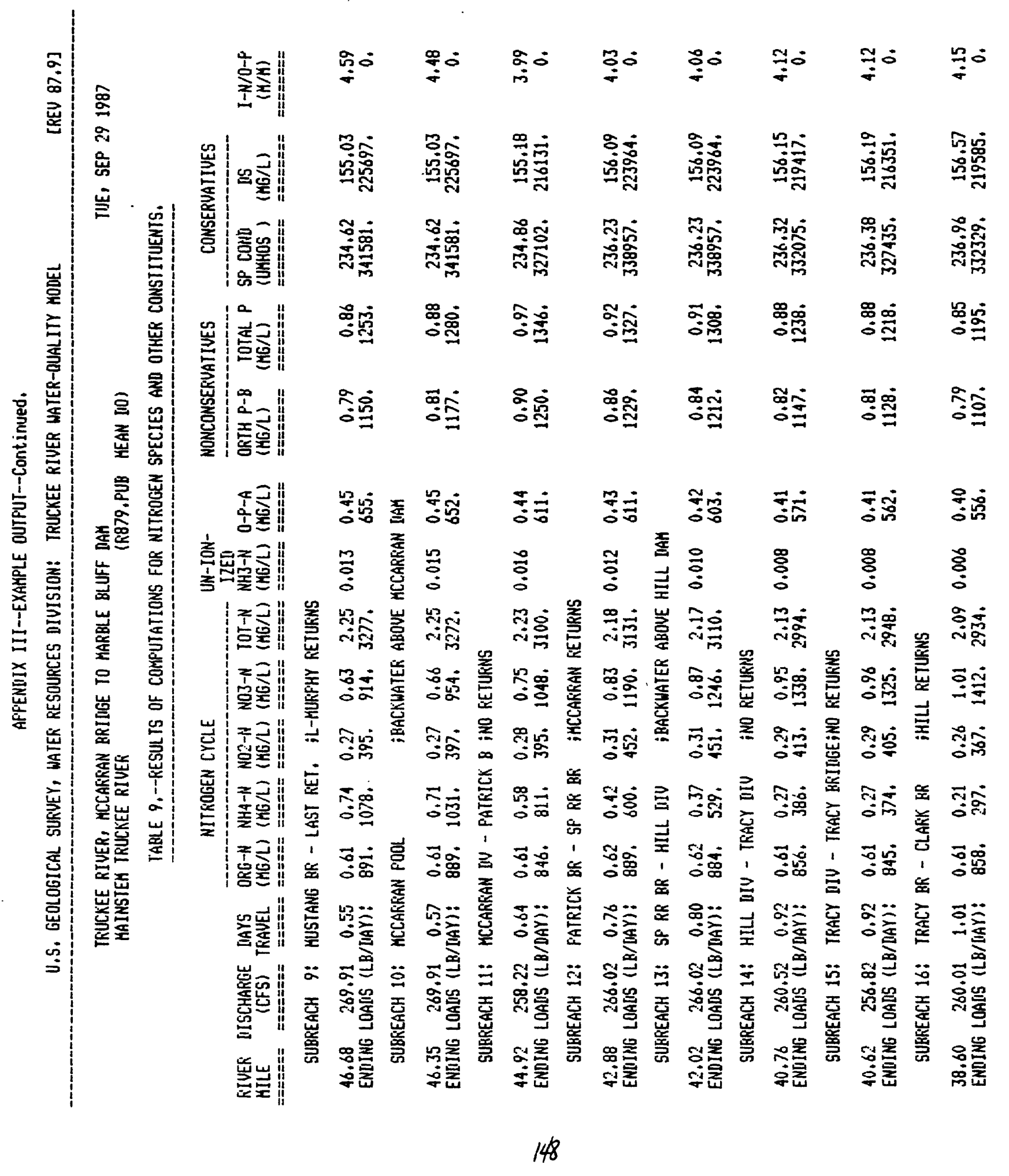




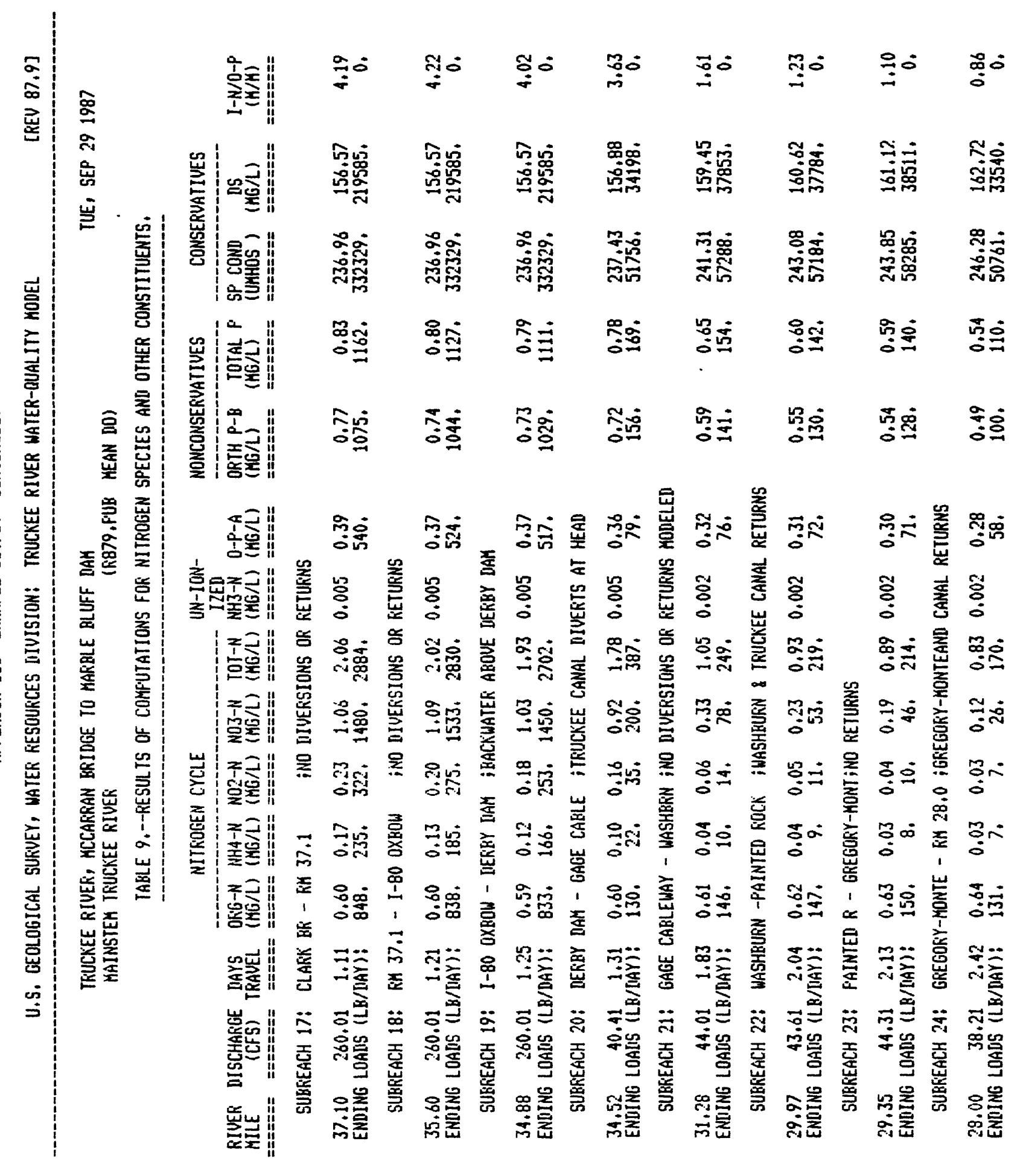




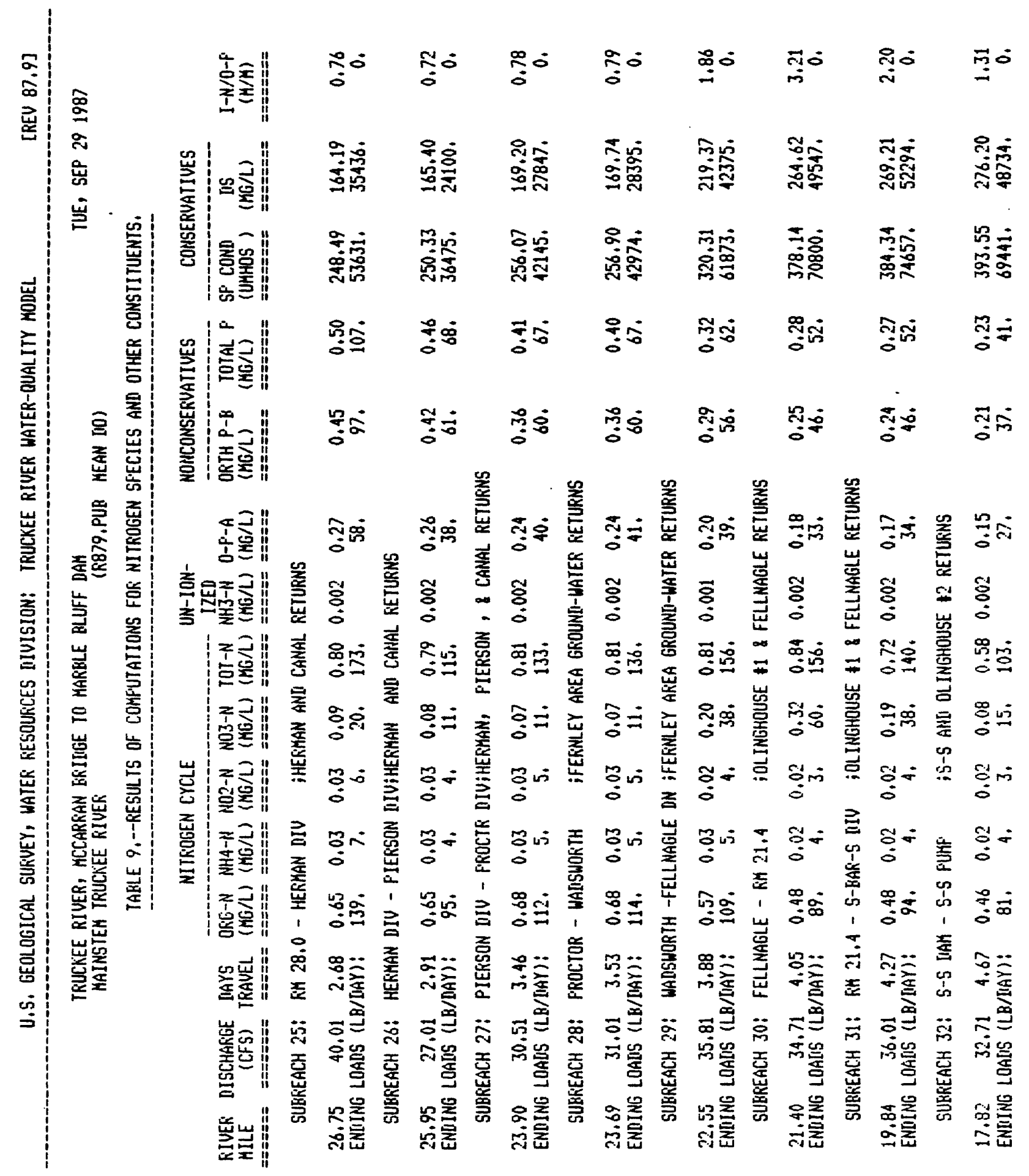




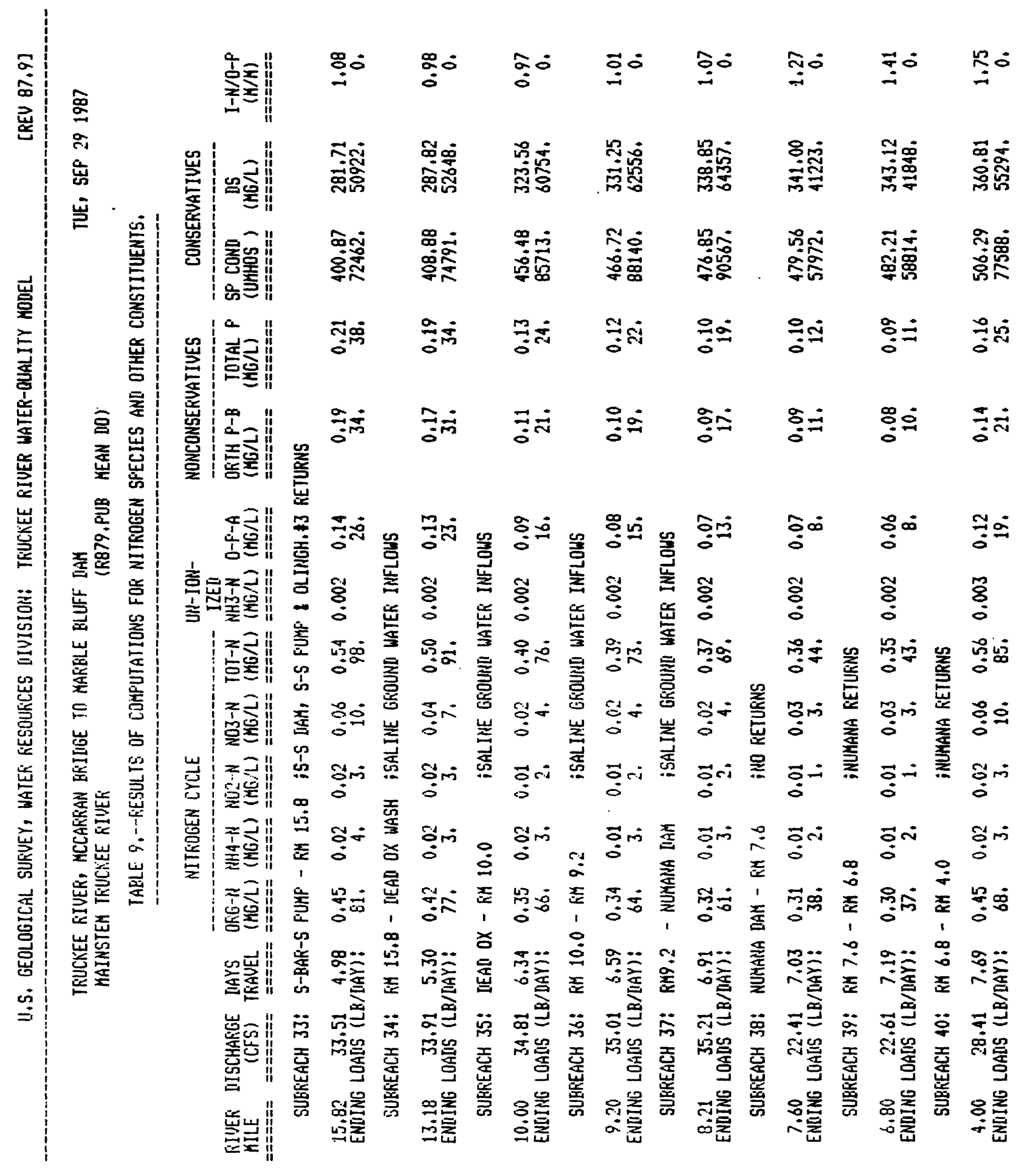




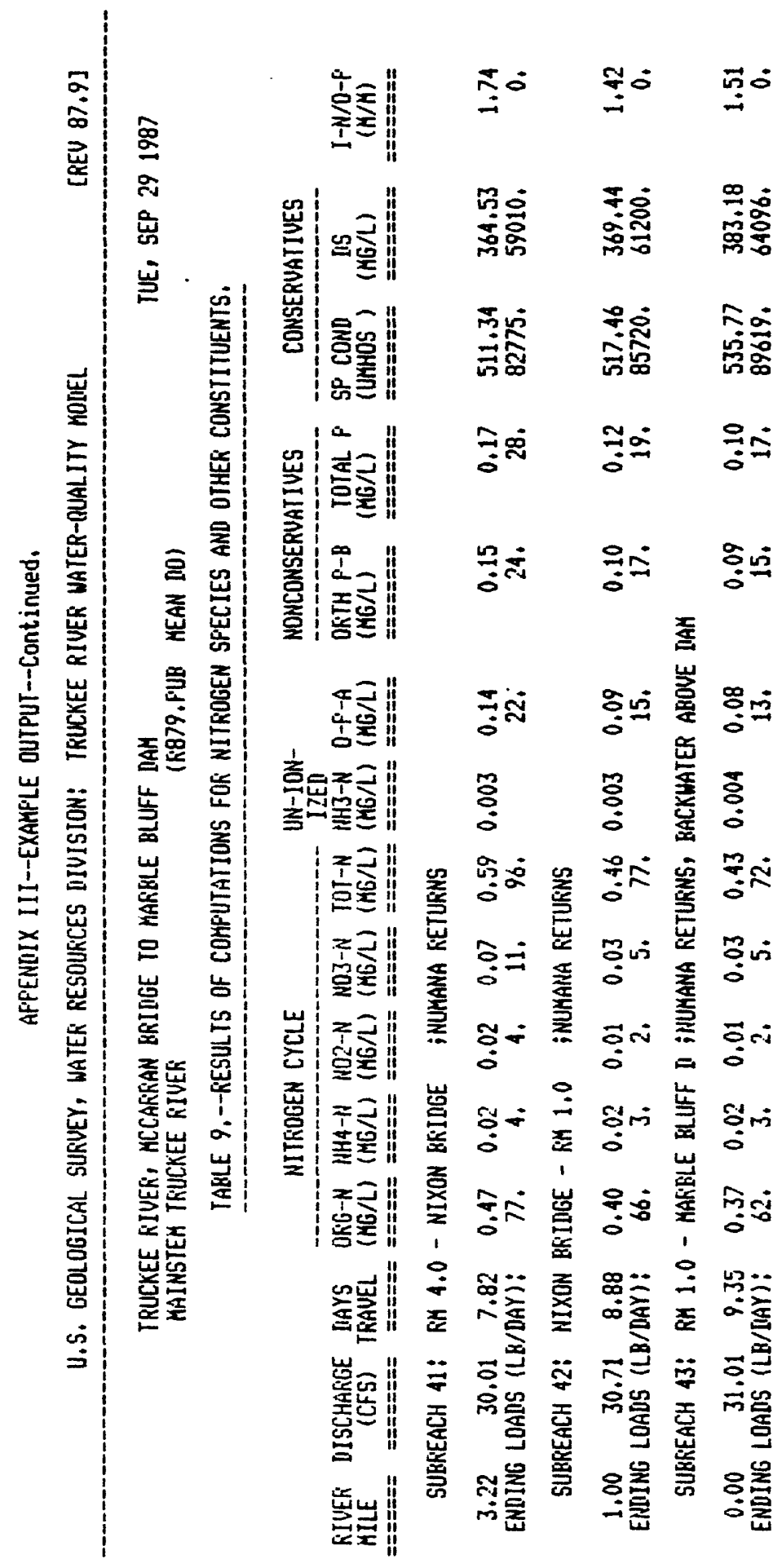




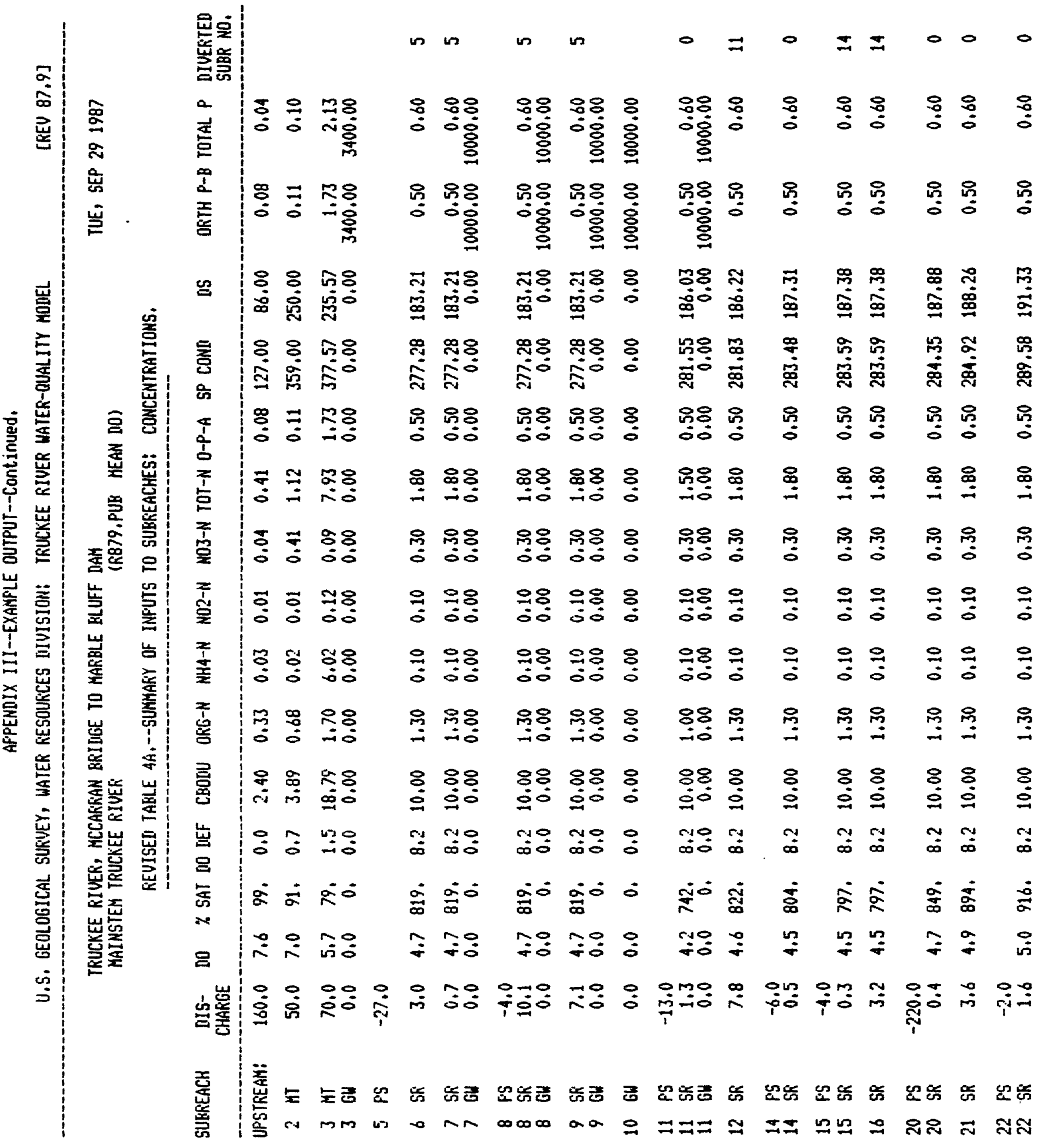




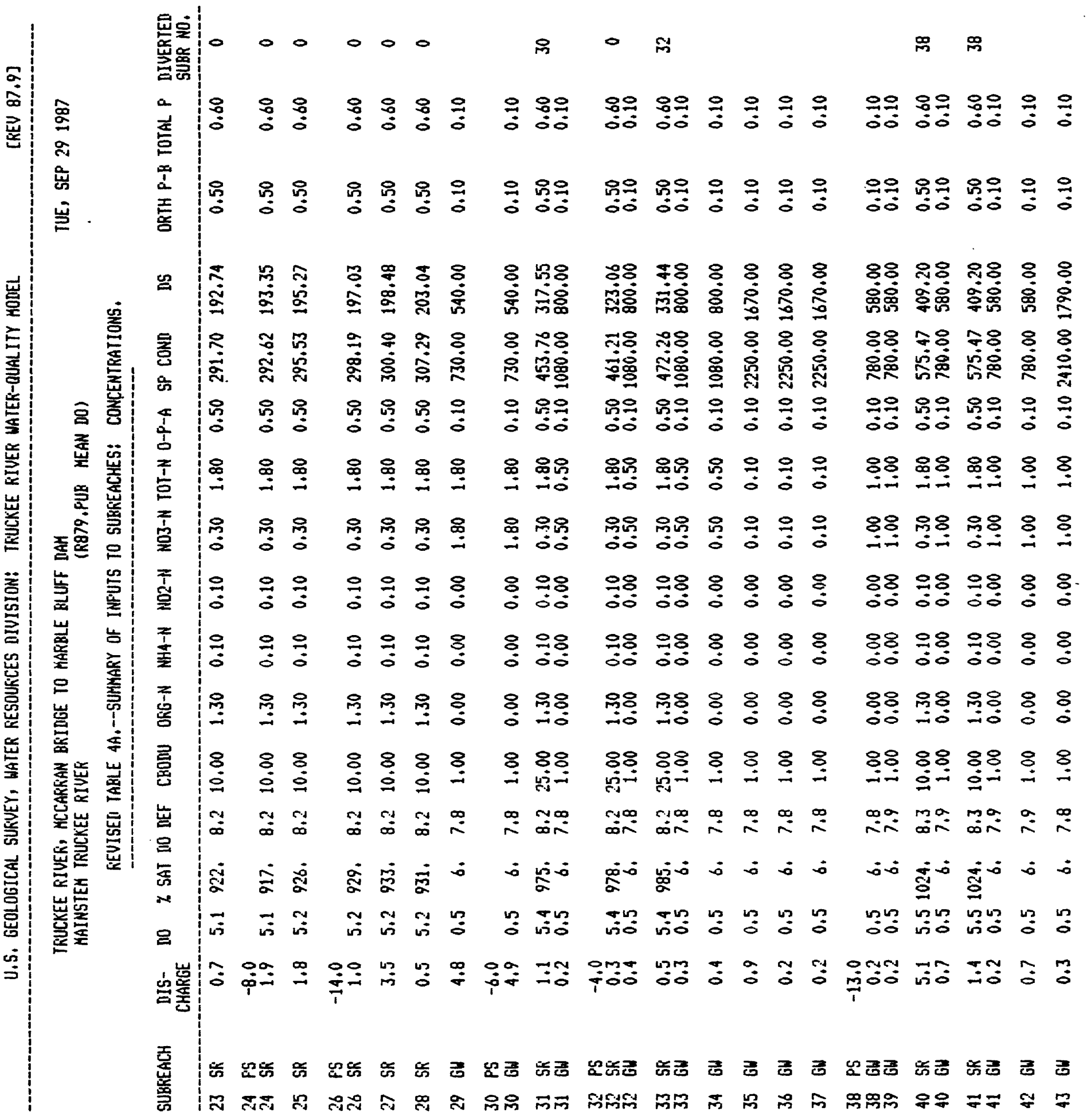




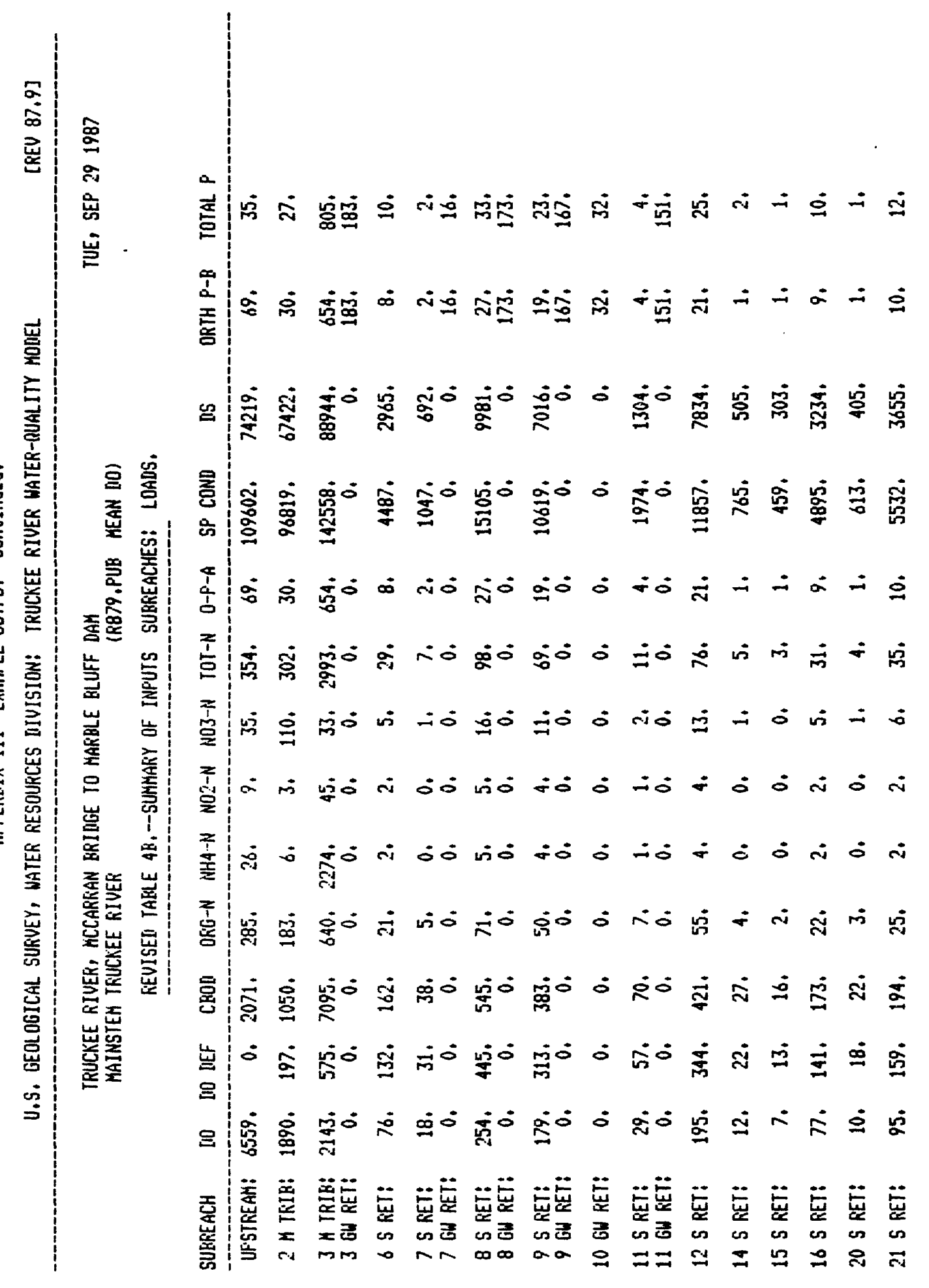




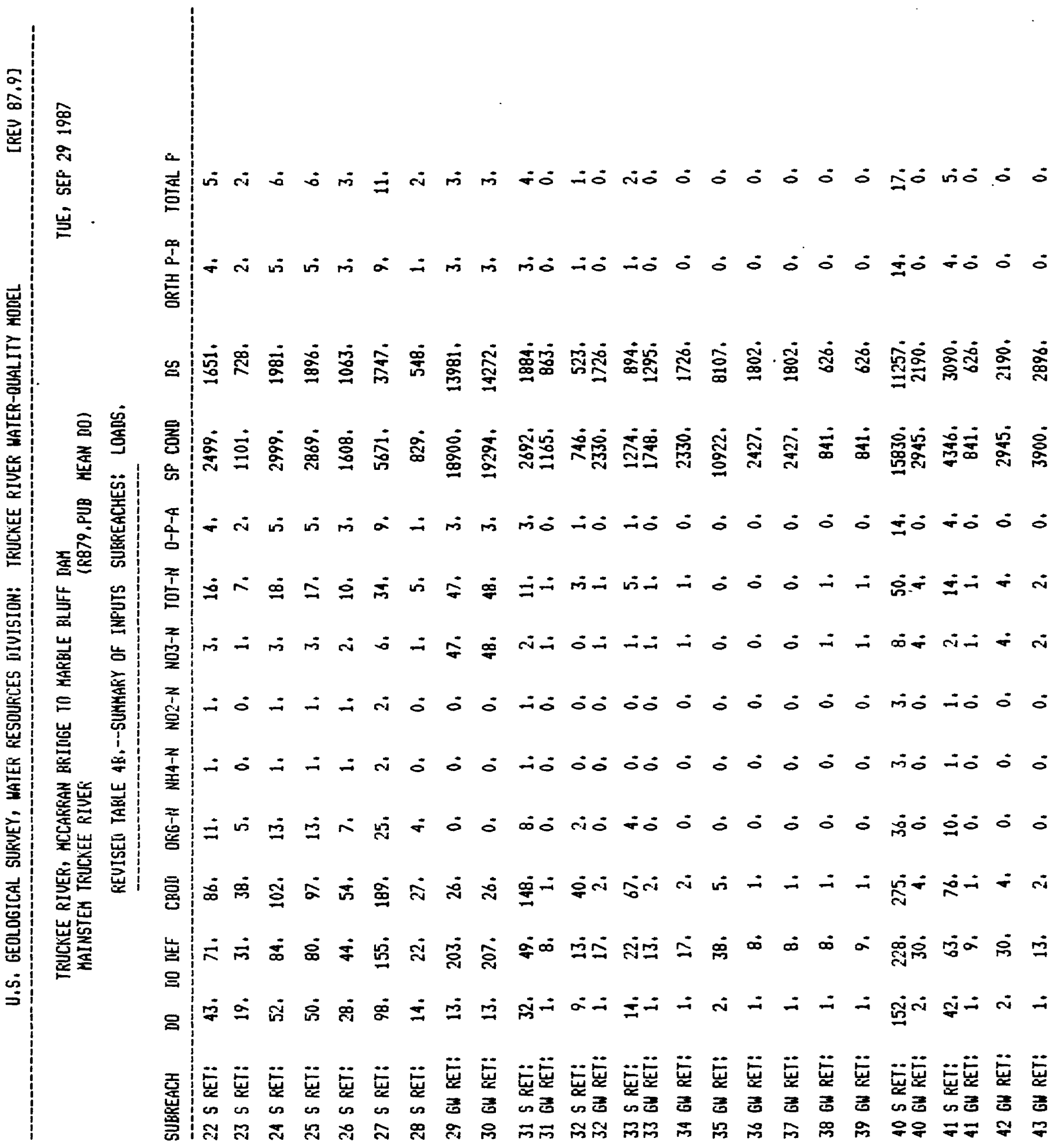


5
0
0
$\overrightarrow{3}$

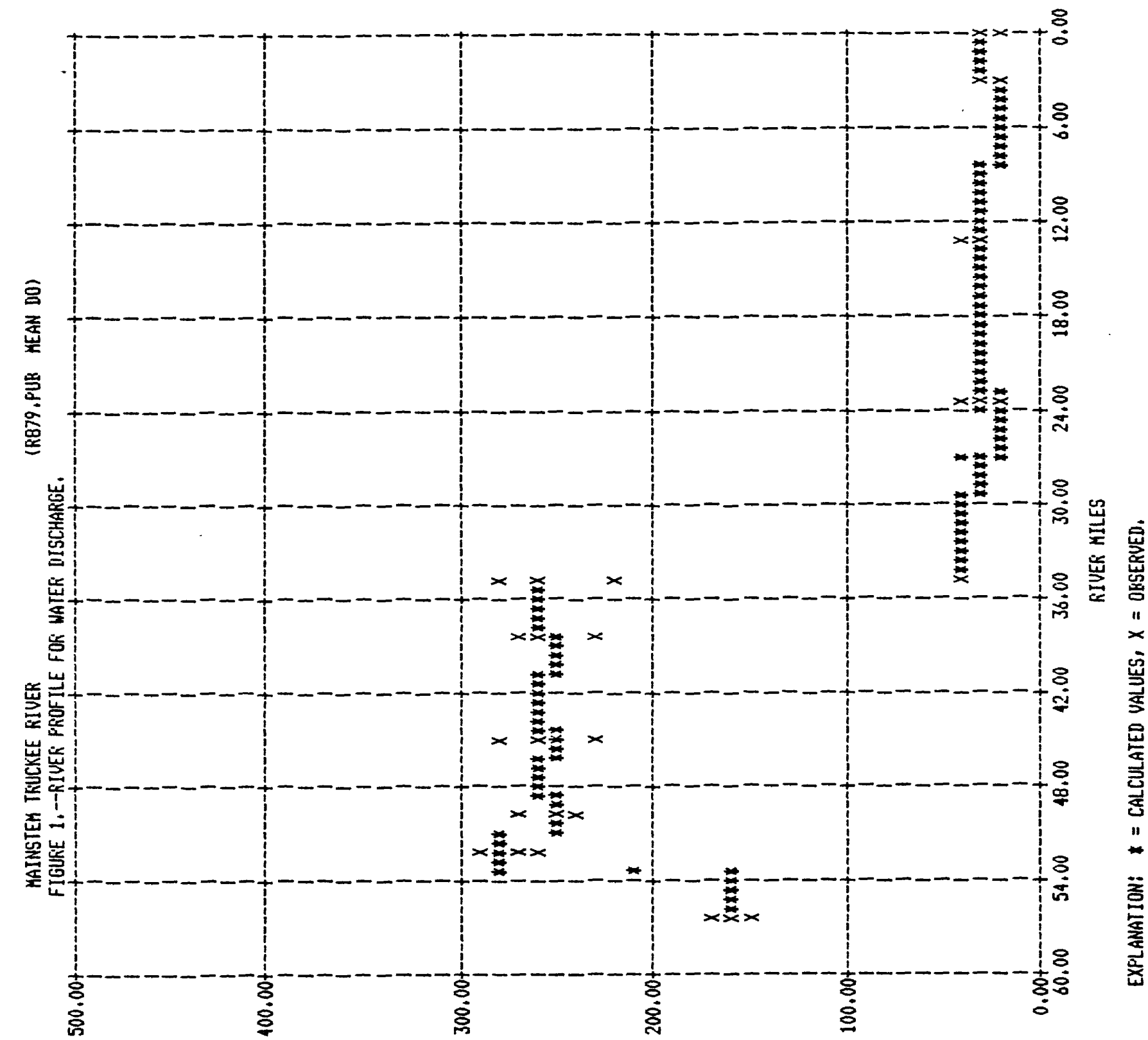

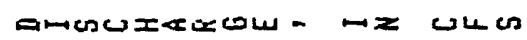




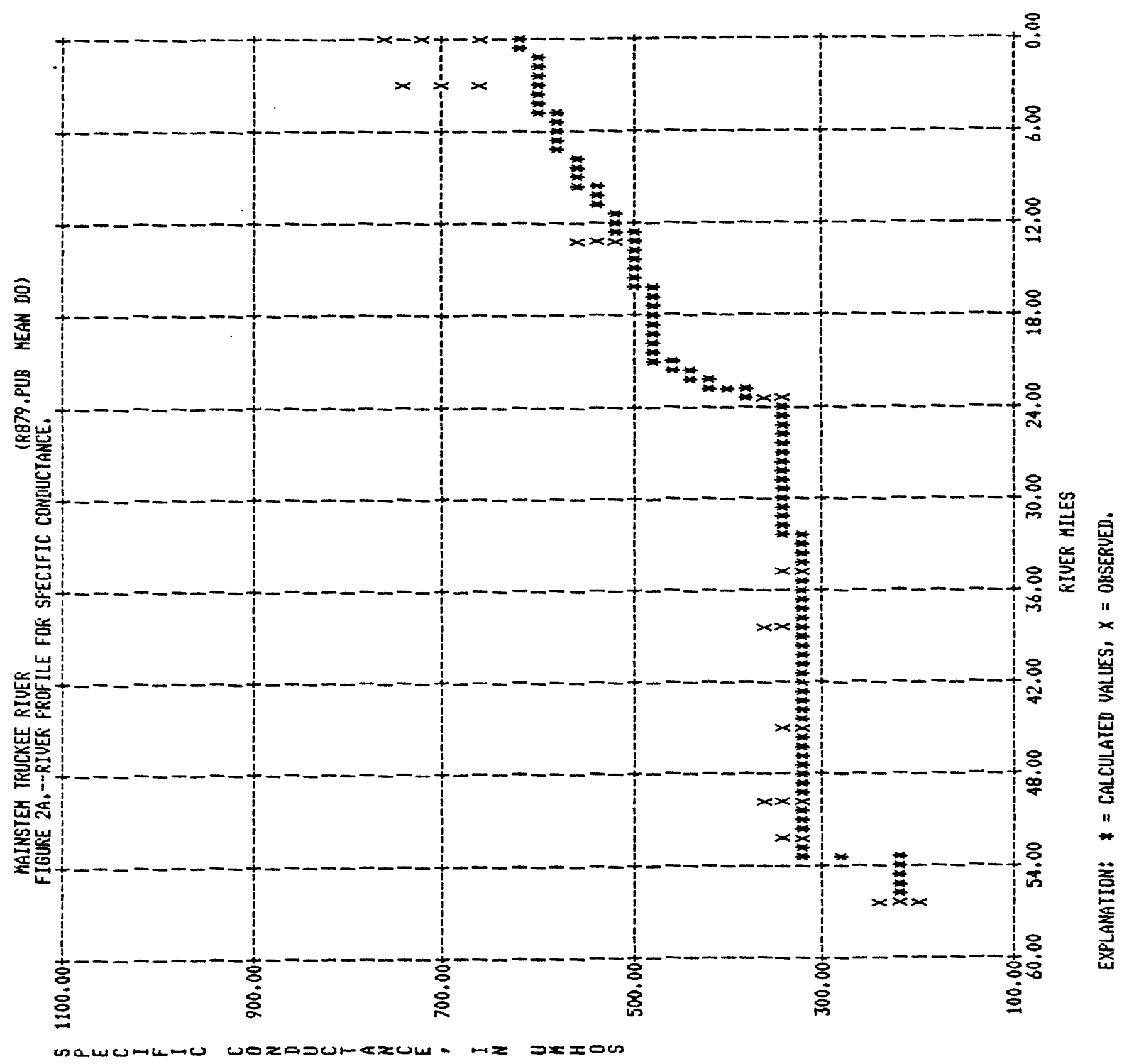




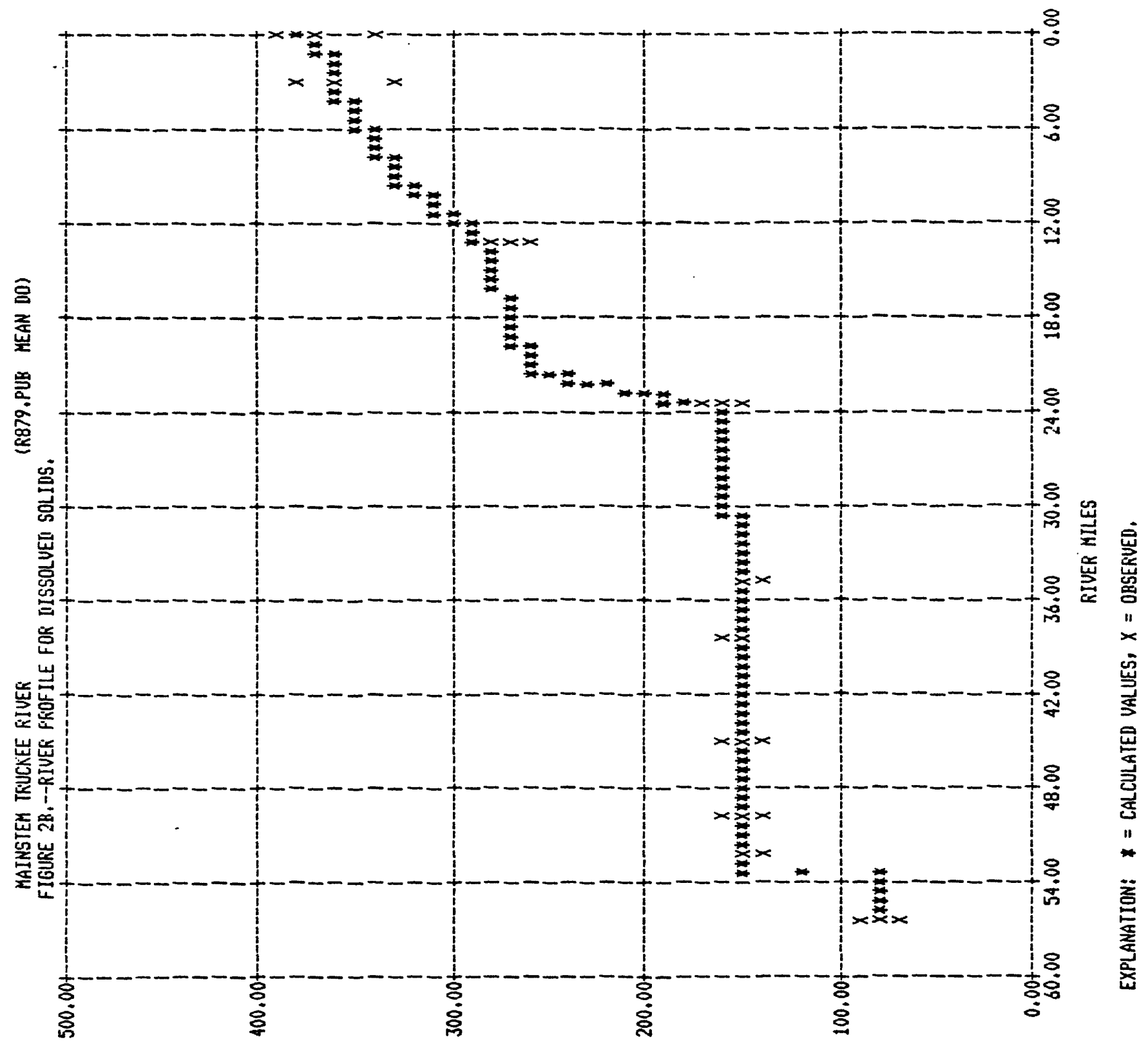

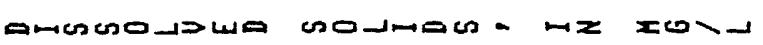




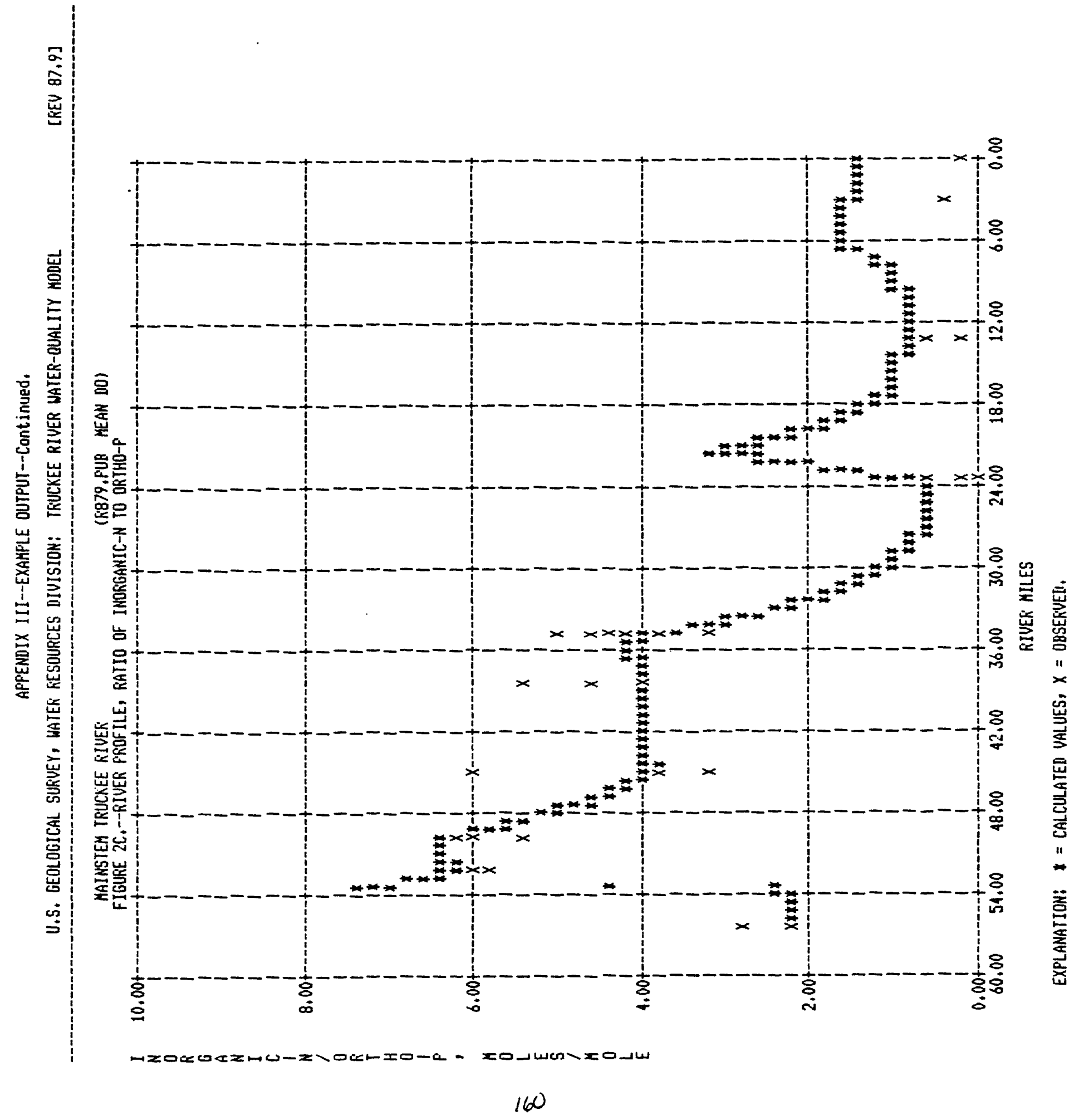




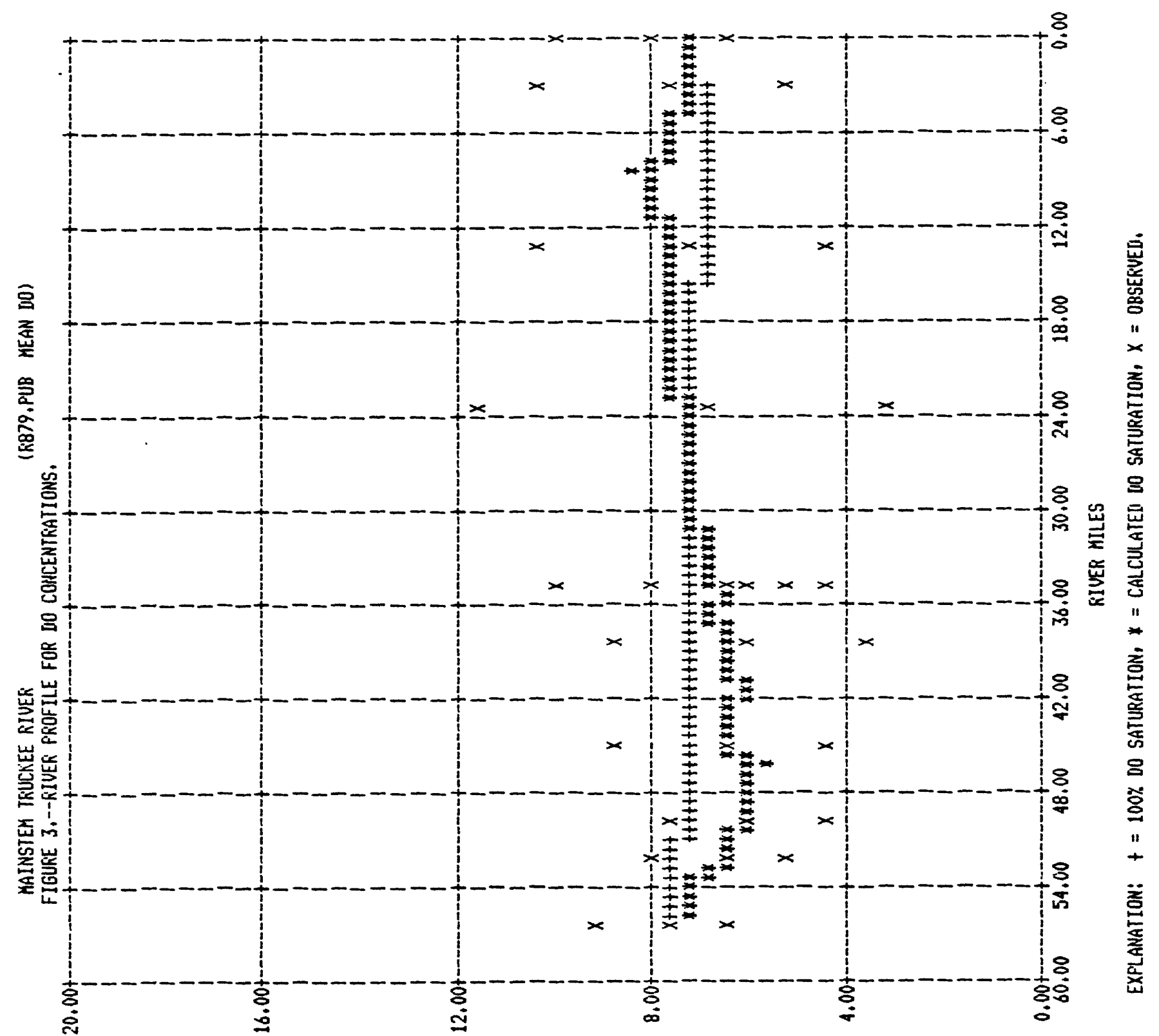

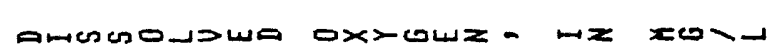




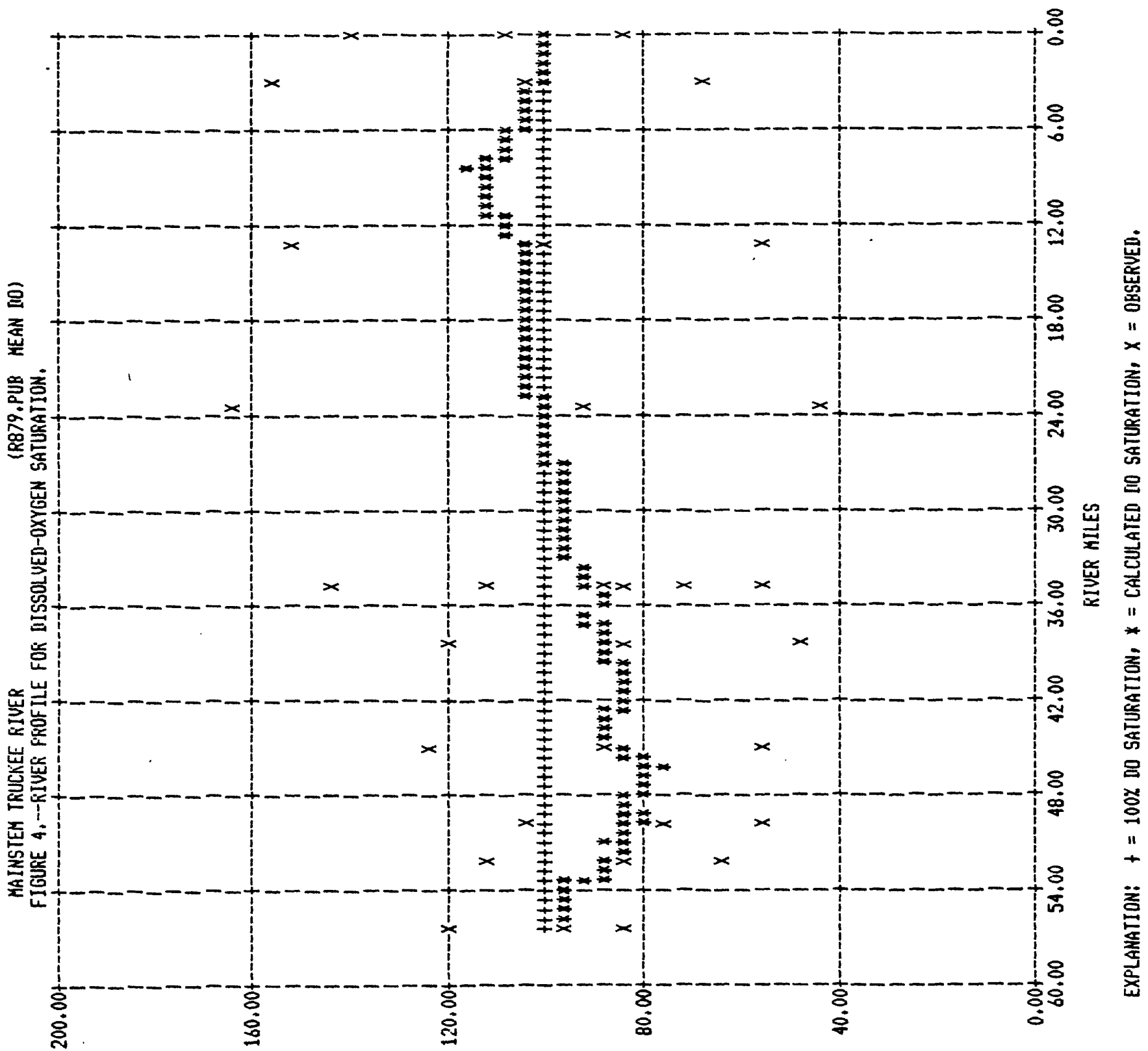

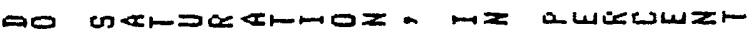




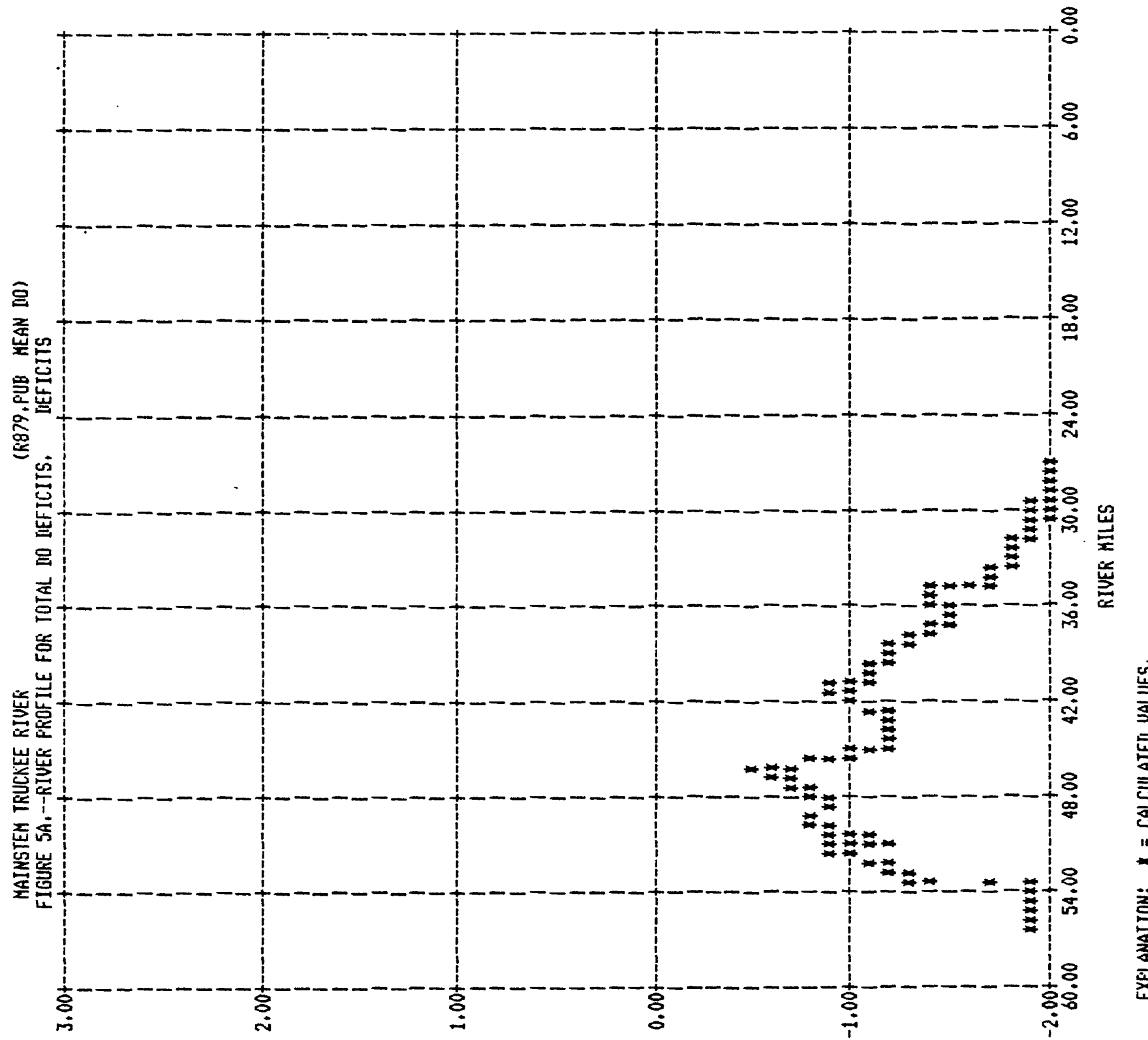

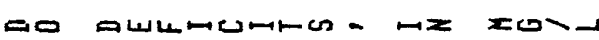




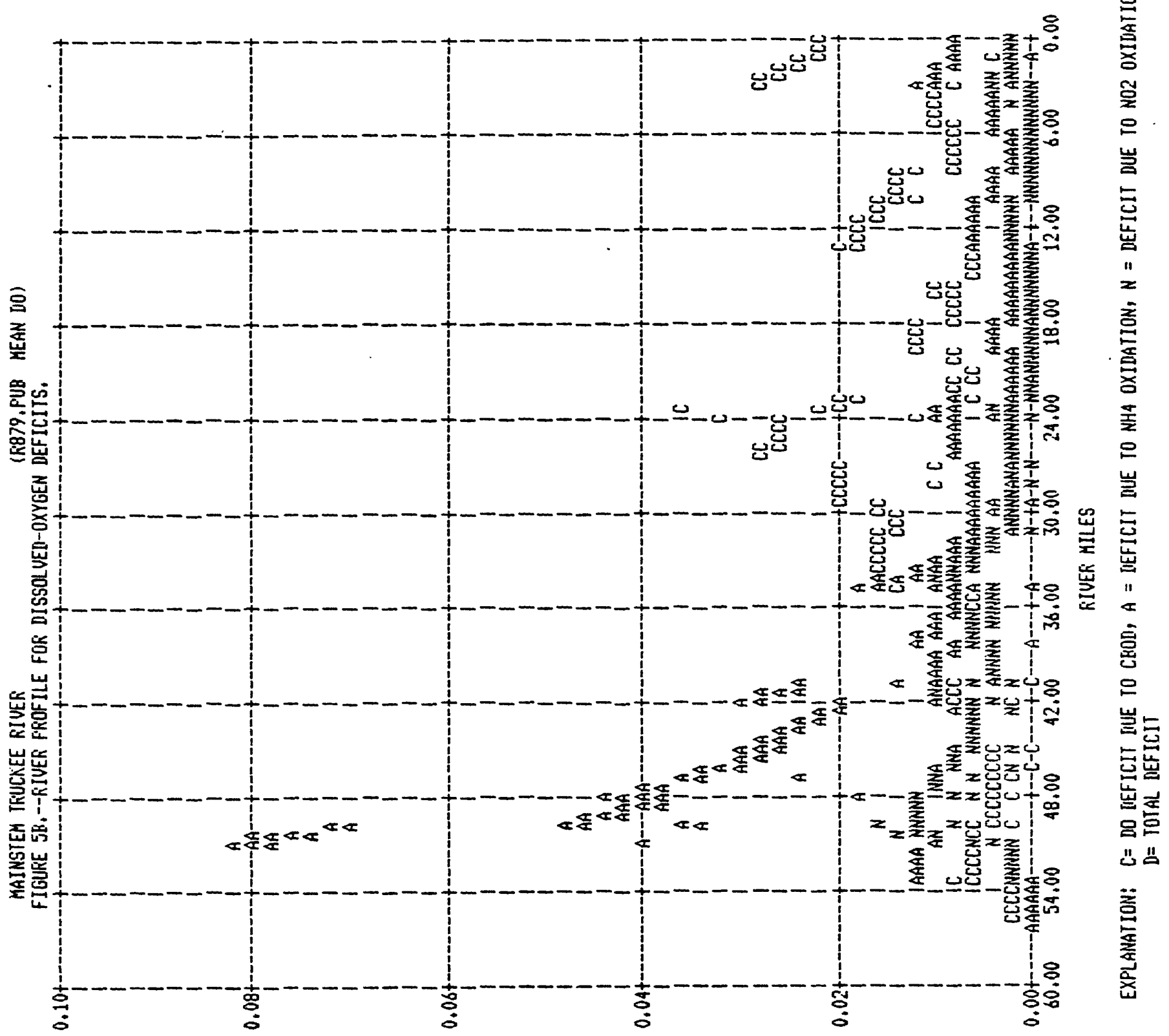

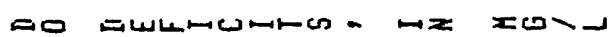




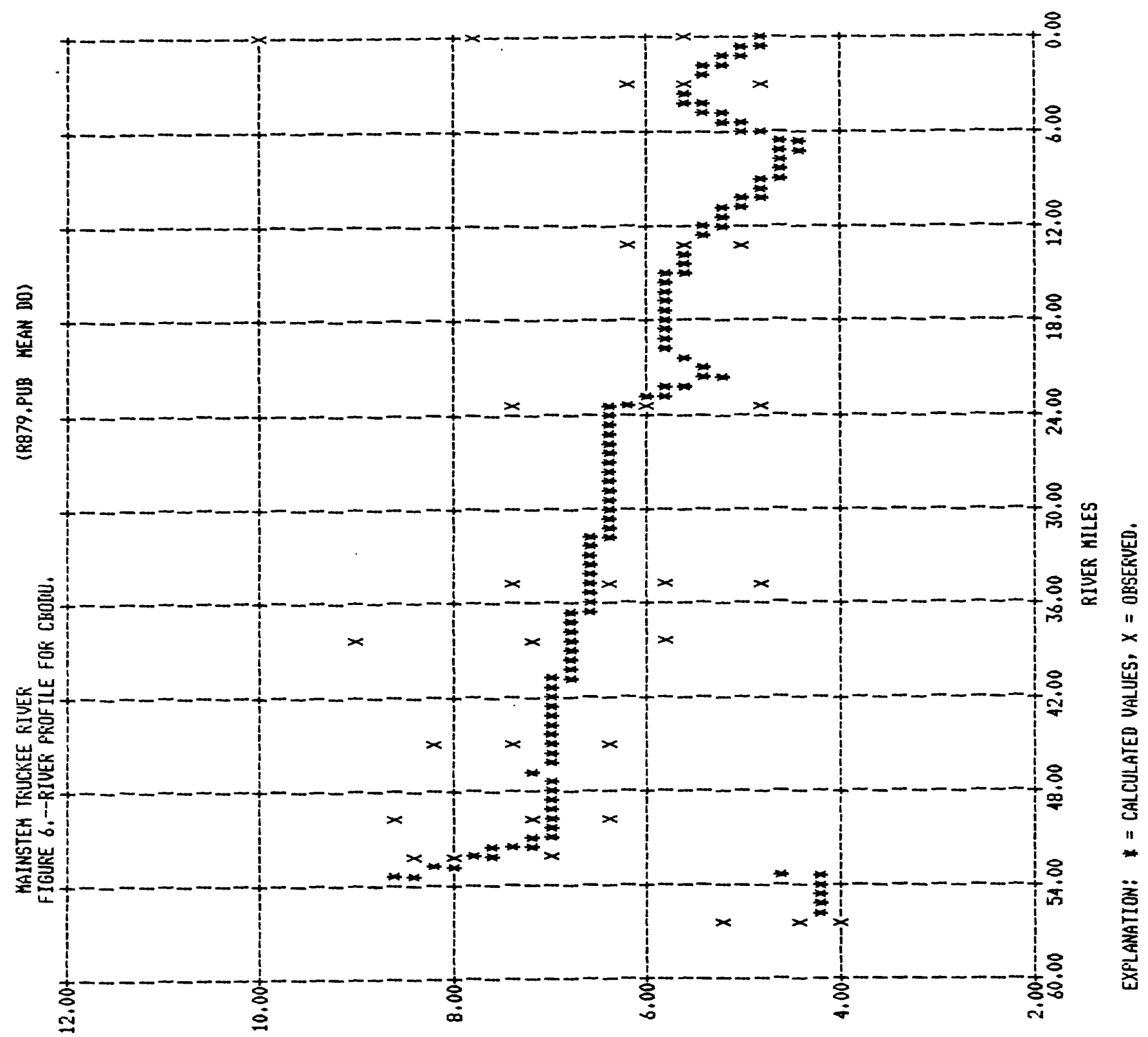

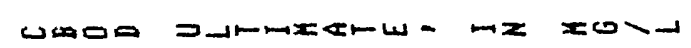


离

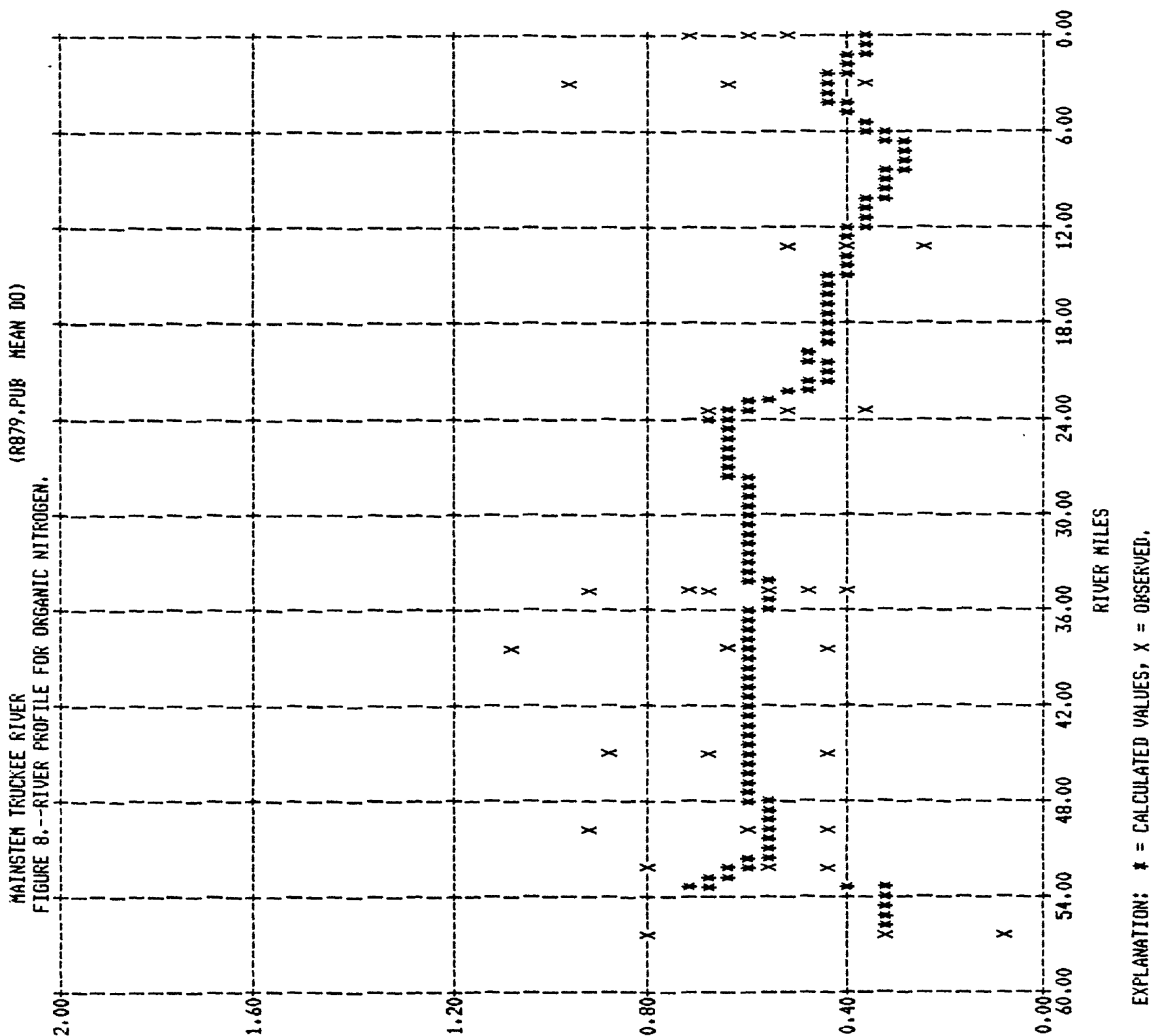

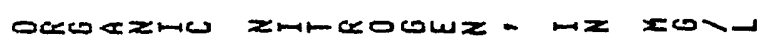




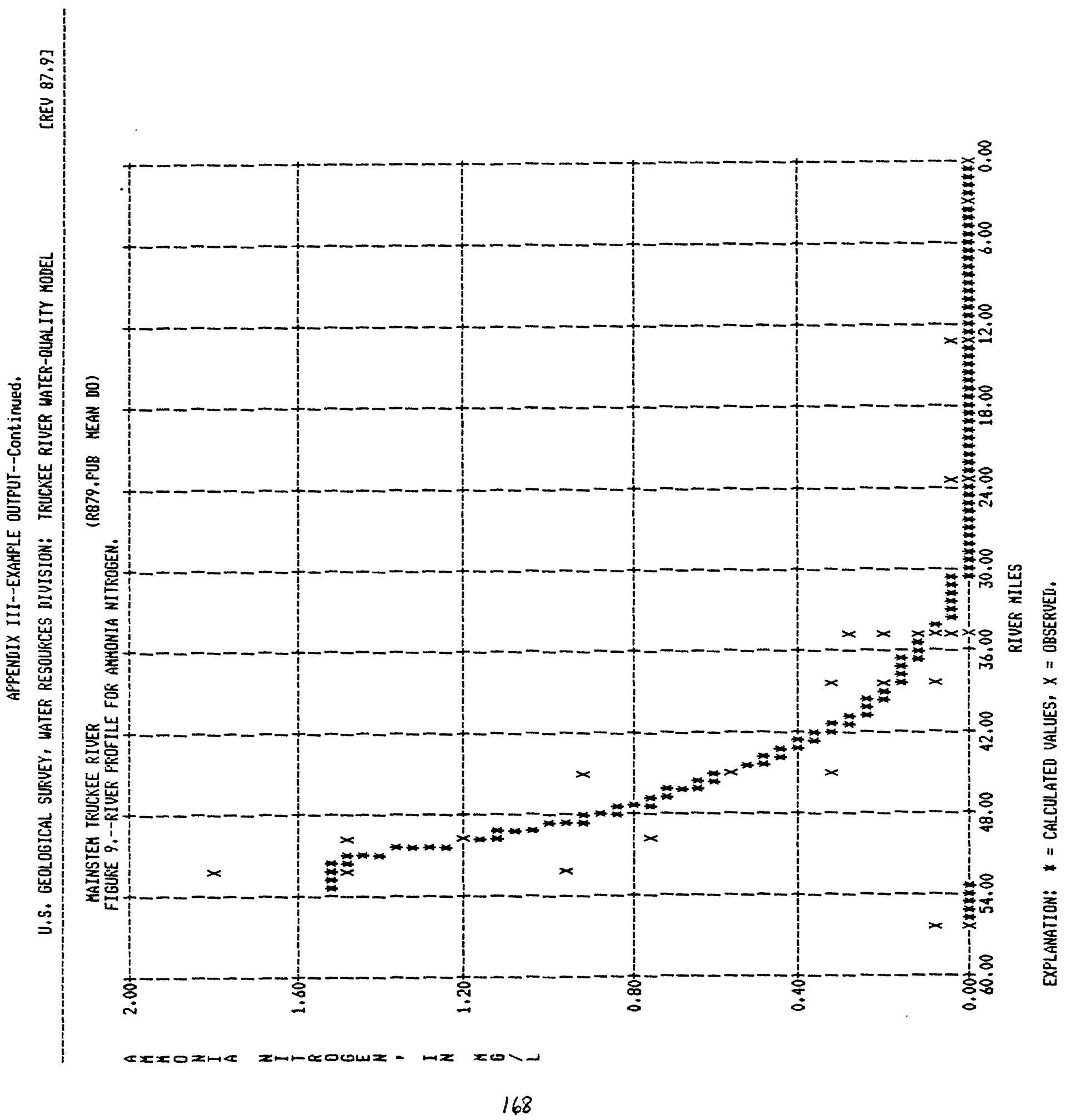




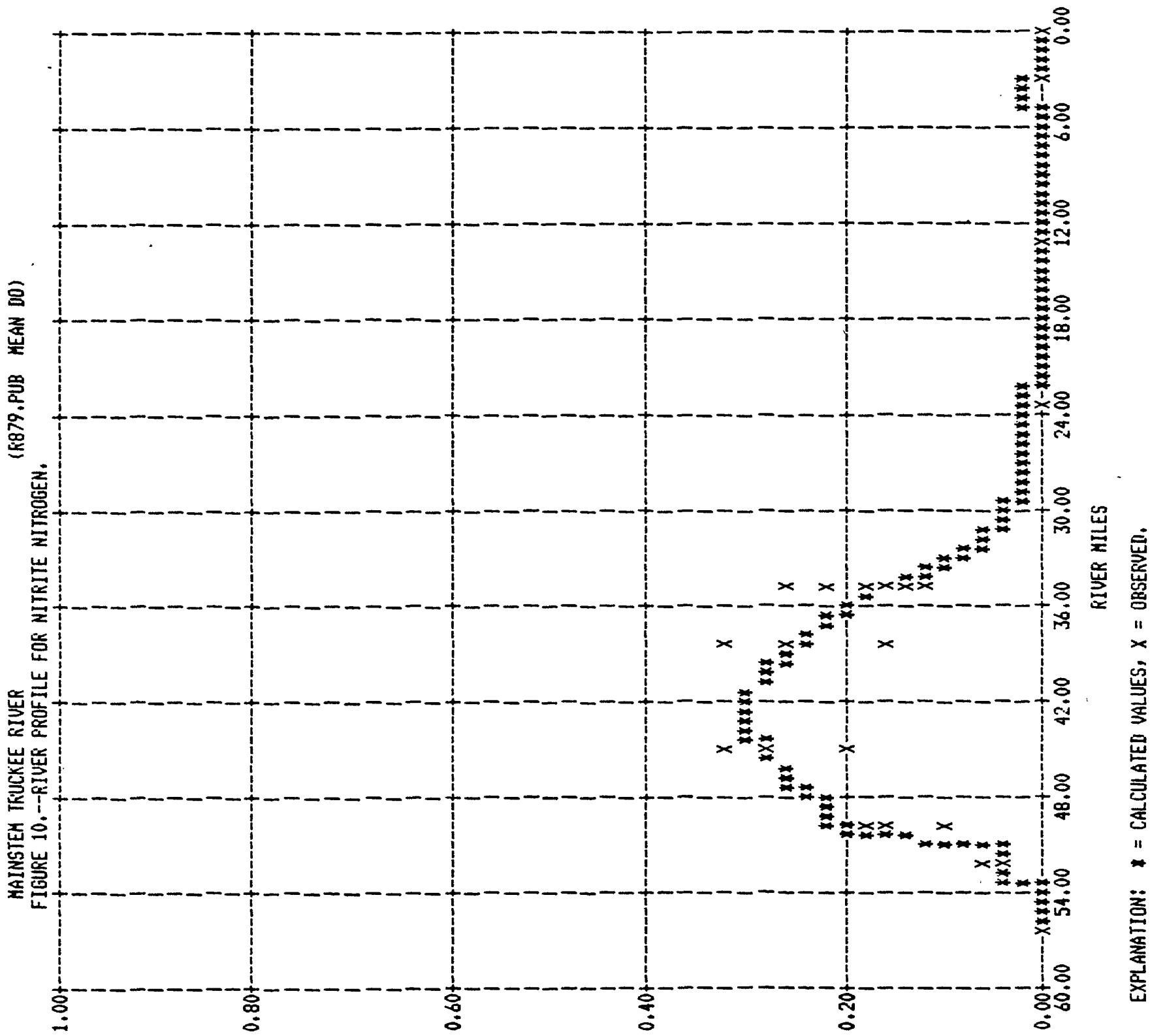

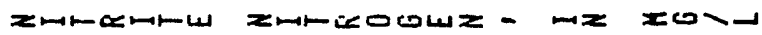




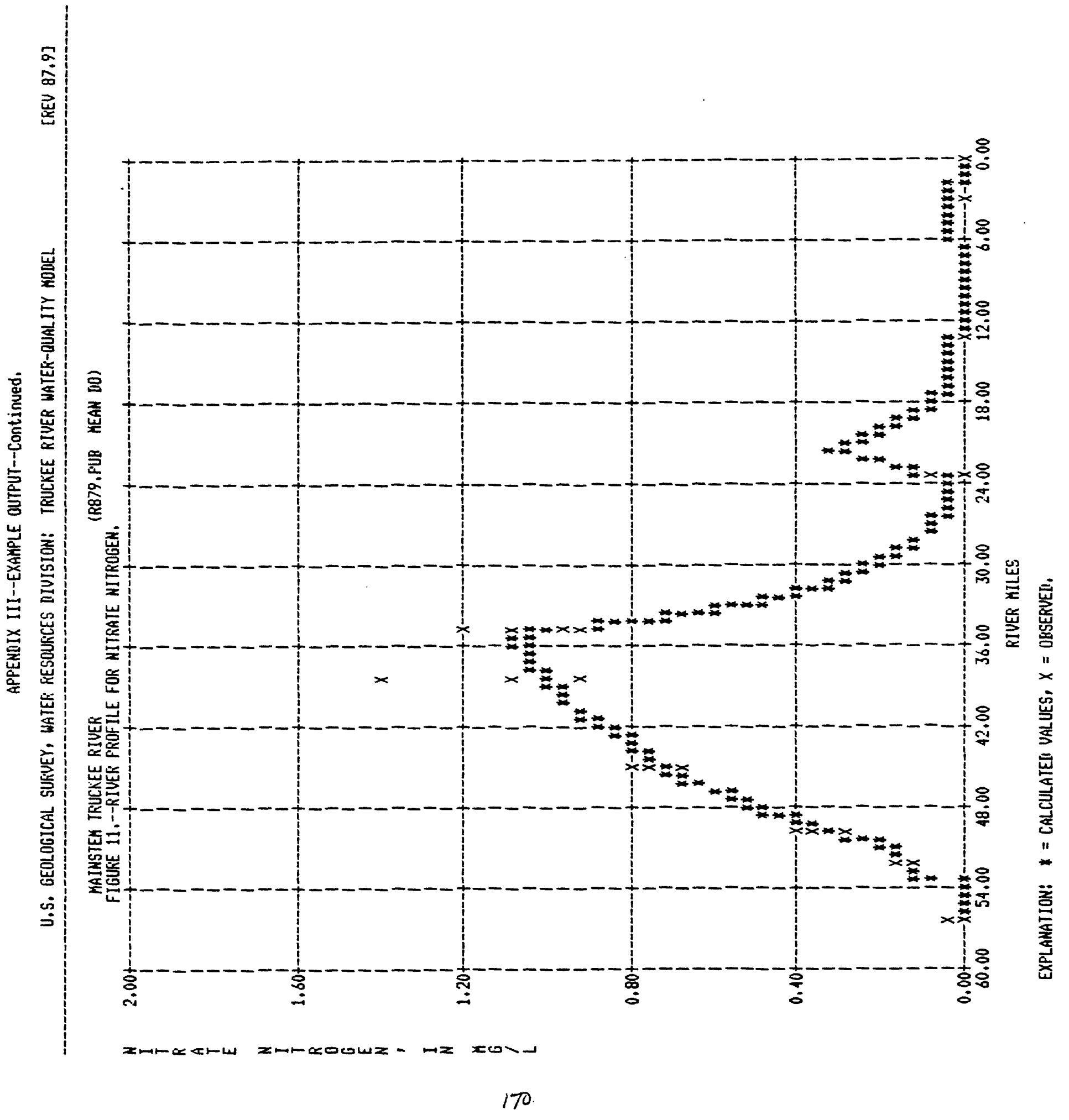




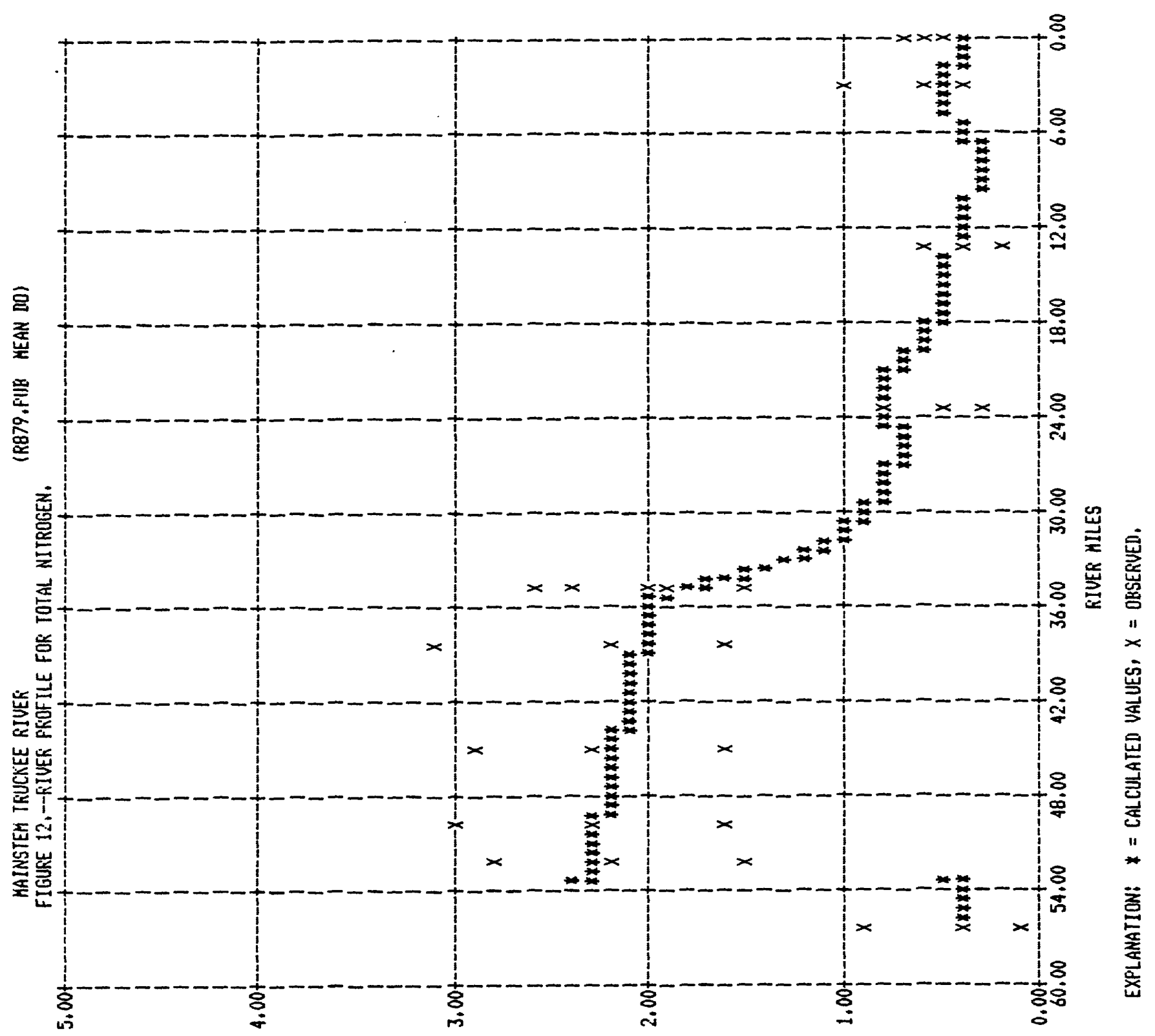

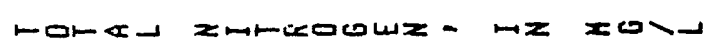




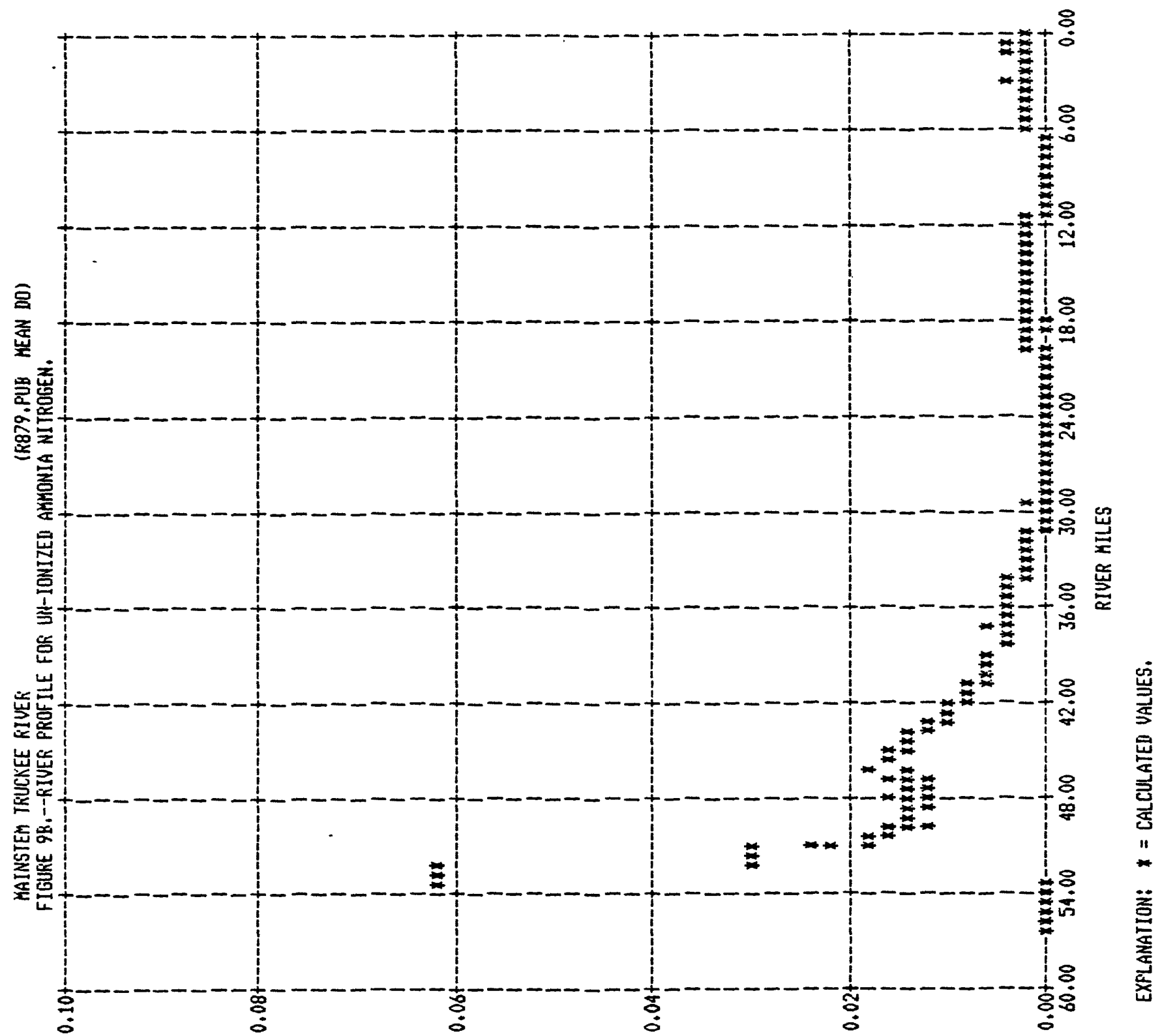

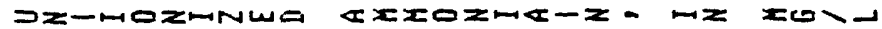




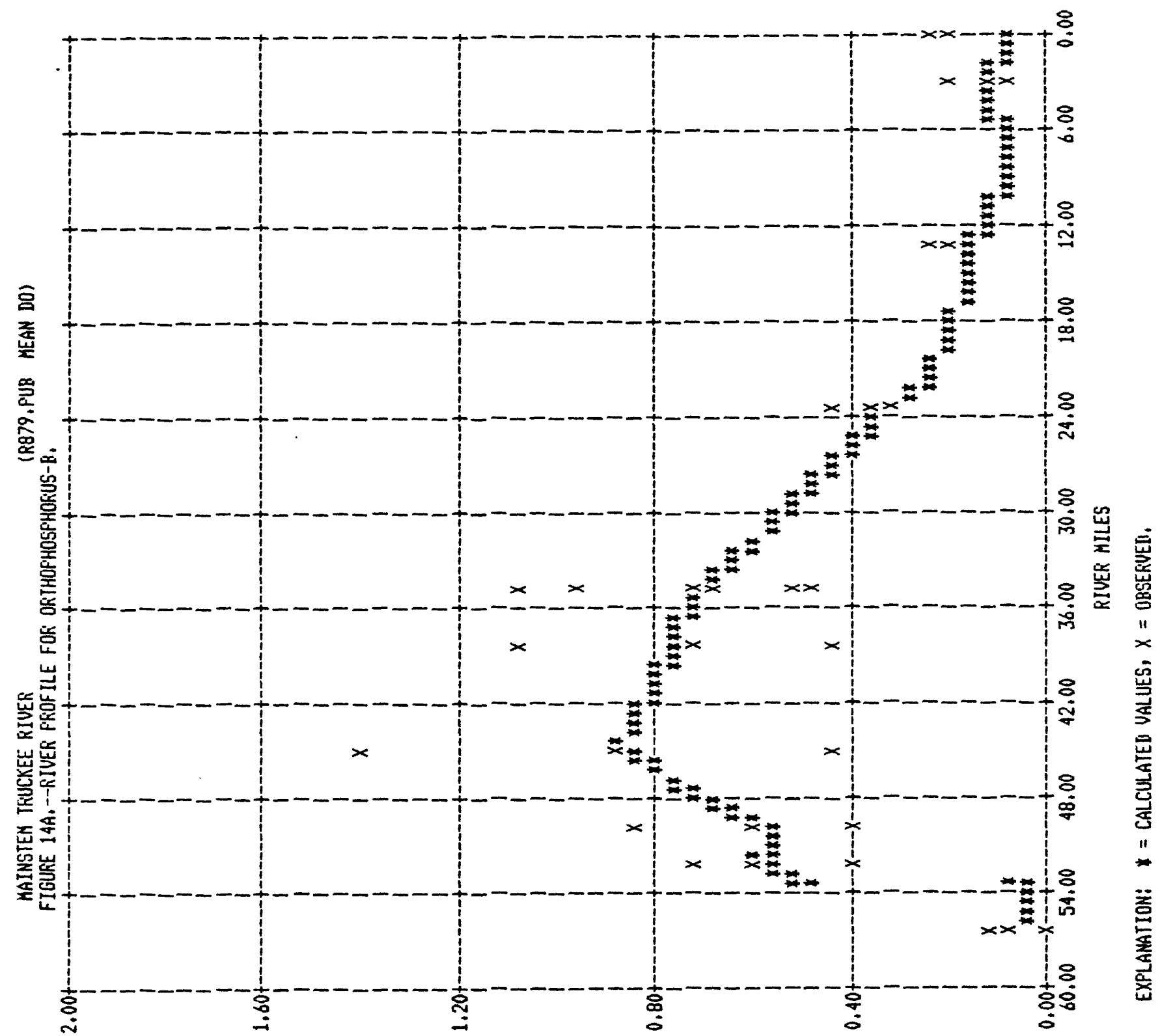

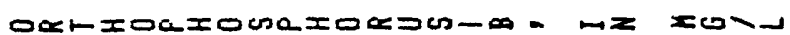




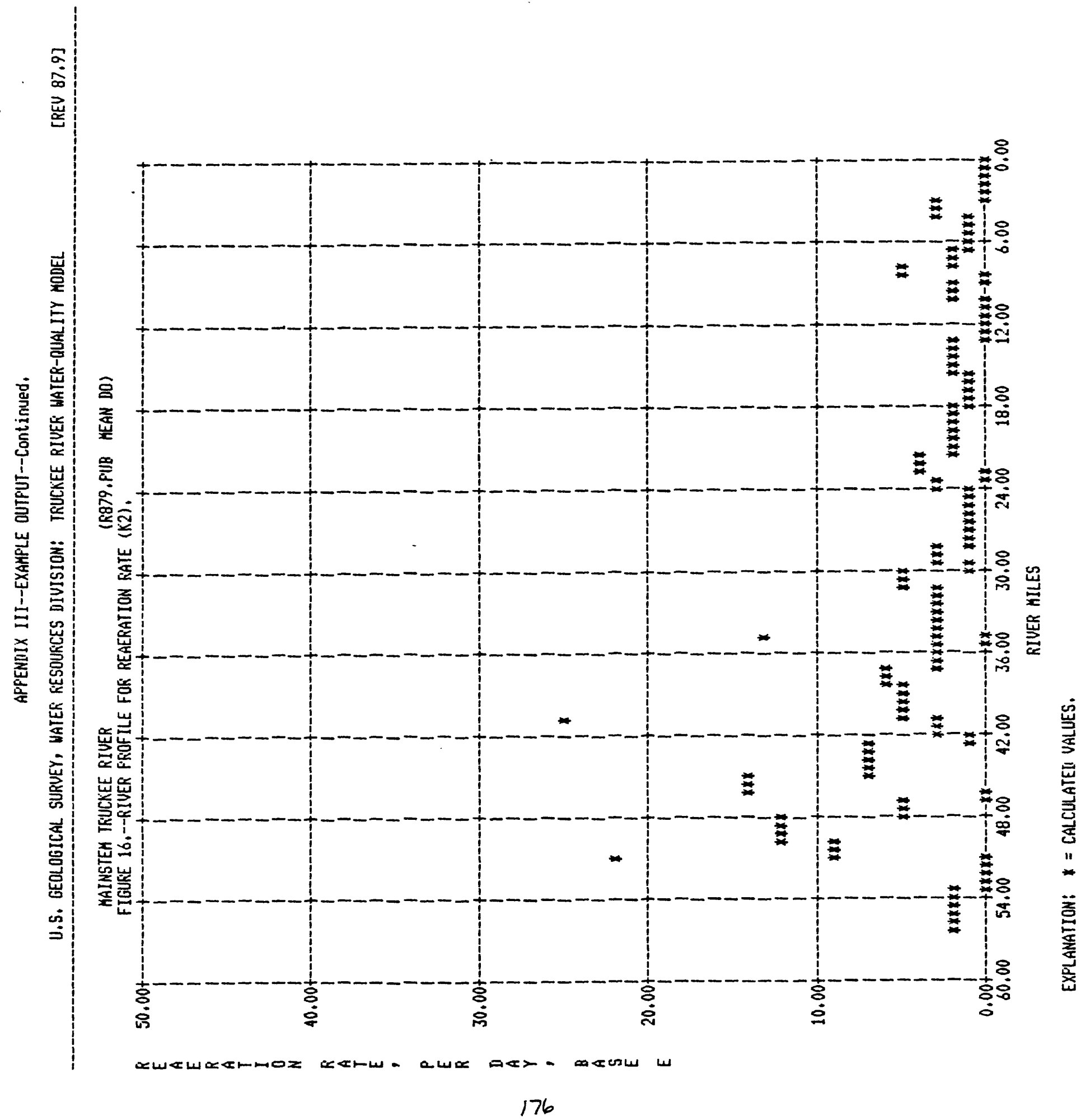




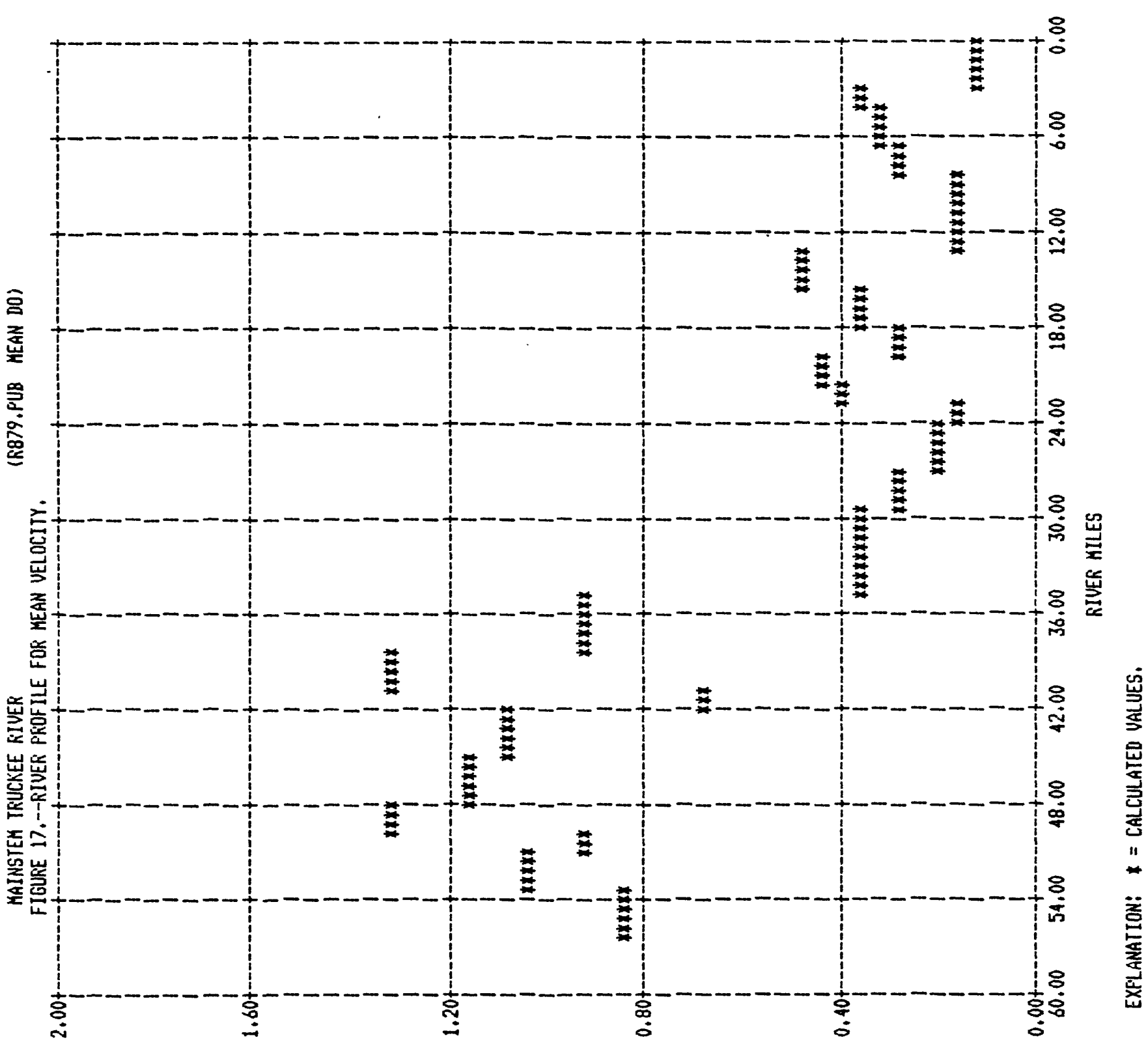

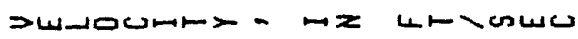


a

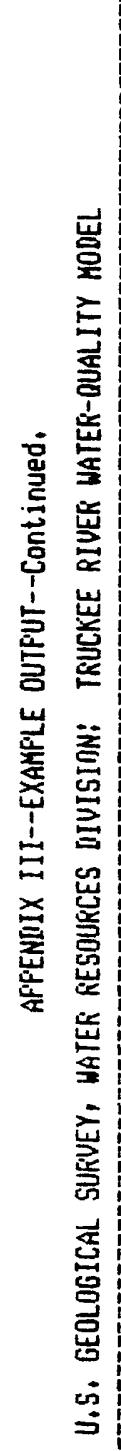

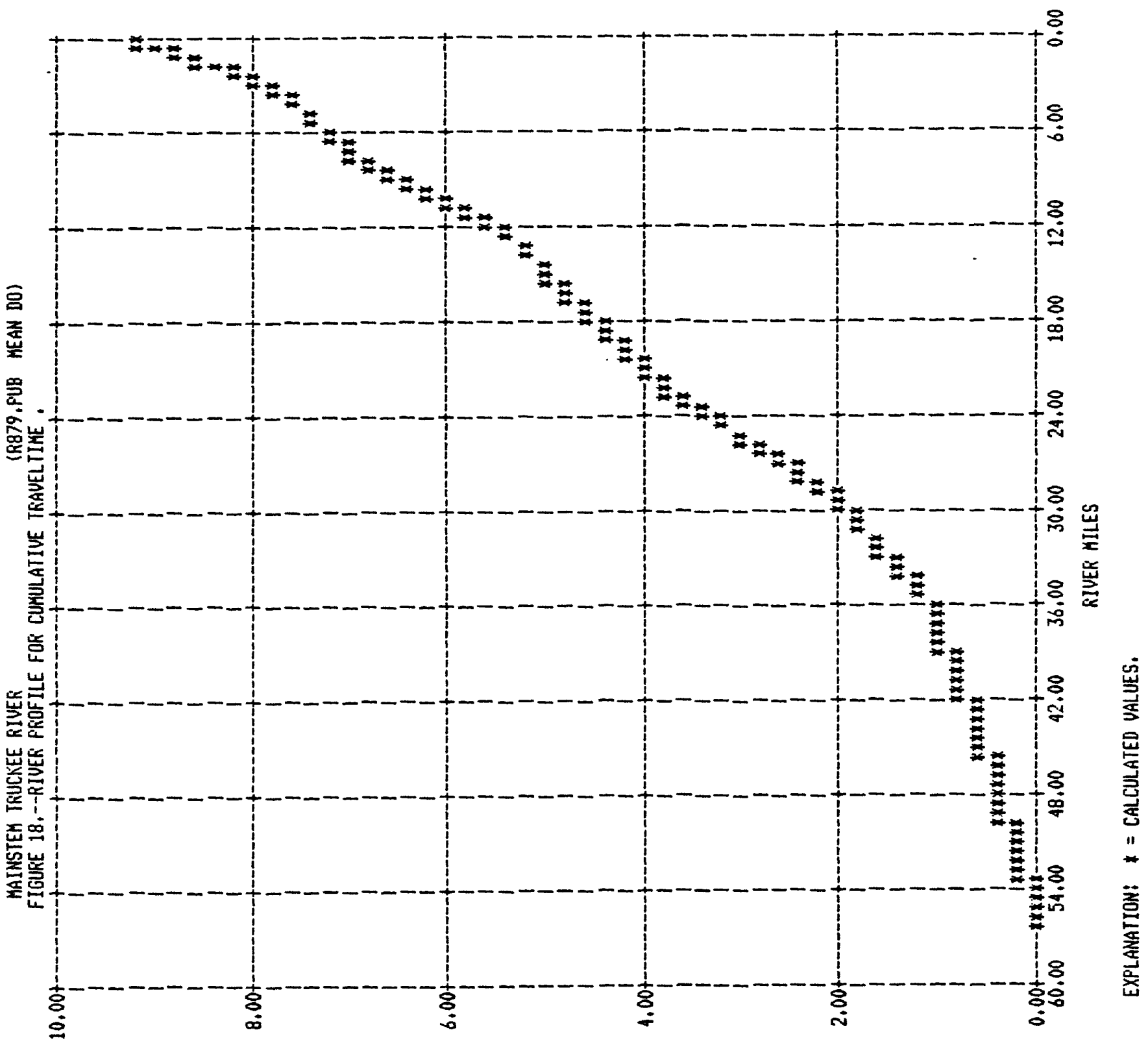

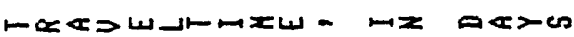




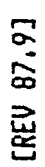

岑

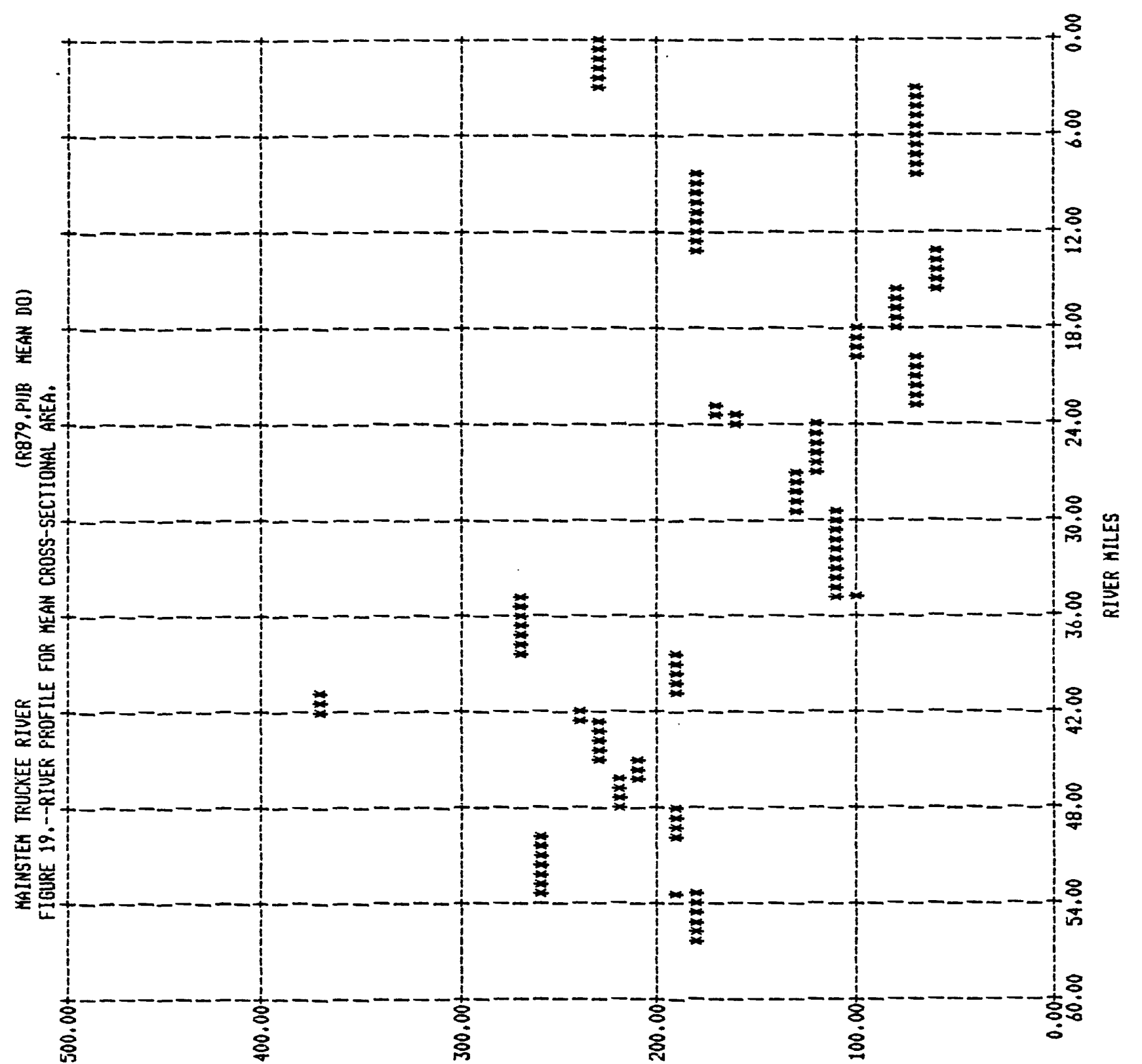

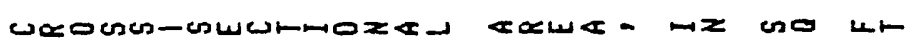




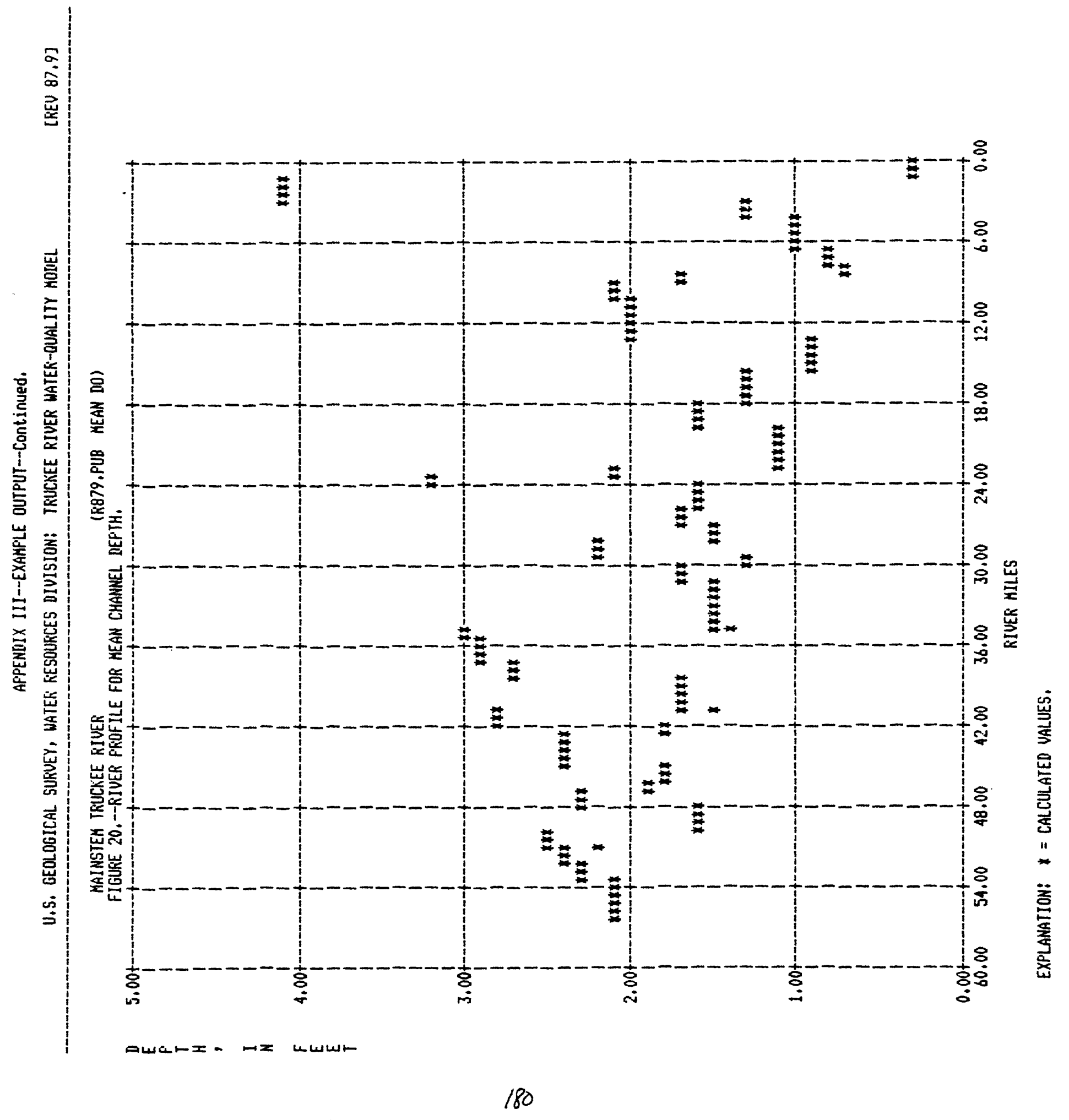




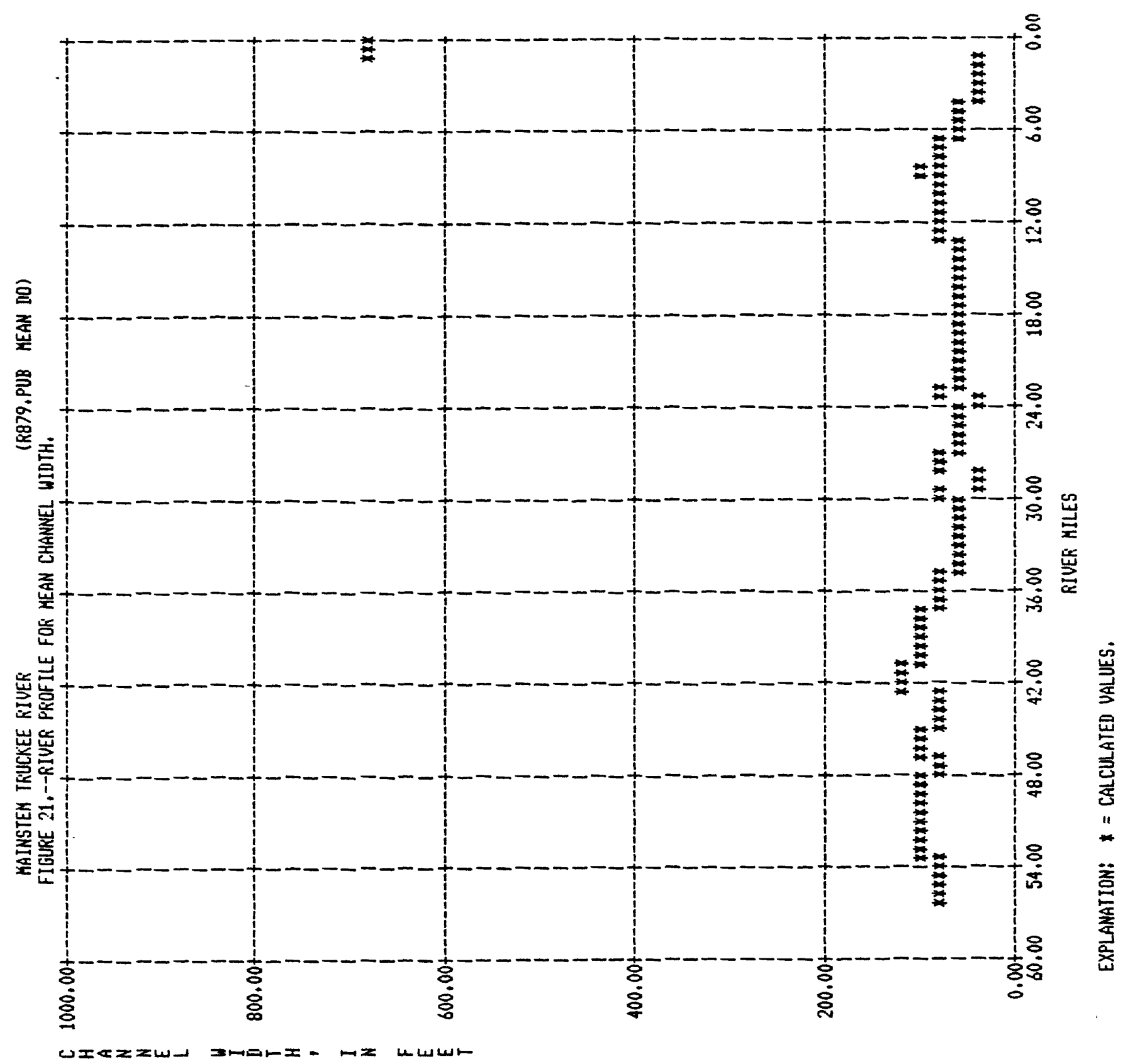

